\title{
Structure-Property Relationships in Ordered Co-Pt Alloys of Near- Eutectoid Compositions
}

A Dissertation
Presented to
the faculty of the School of Engineering and Applied Science
University of Virginia

in Partial Fulfillment

of the requirements for the Degree

Doctor of Philosophy (Materials Science and Engineering)

by

Priya Ghatwai

May 2015 


\title{
Approval Sheet
}

The dissertation is submitted in partial fulfillment of the

$$
\text { requirements for the degree of }
$$

Doctor of Philosophy (Materials Science and Engineering)

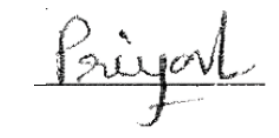

PRIYA GHATWAI

Author

This dissertation has been read and approved by the examining committee:

\author{
JERROLD A. FLORO
}

Advisor

WILLIAM A. SOFFA

\section{WILLIAM C. JOHNSON}

JAMES M. HOWE

PATRICK E. HOPKINS

Accepted for the School of Engineering and Applied Science:

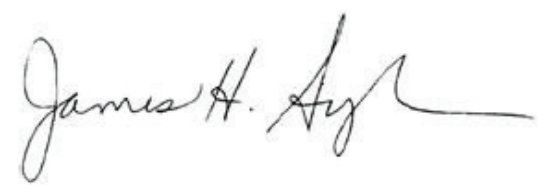

Dean, School of Engineering and Applied Science

May 2015 


\begin{abstract}
Co-Pt alloys with compositions in the narrow eutectoid range $\left(\sim \mathrm{Co}_{40} \mathrm{Pt}_{60}\right)$ can undergo eutectoid transformation via the pseudospinodal decomposition mechanism to produce a highly regular nanochessboard structure. This microstructure comprises the hard magnetic $\mathrm{L} 1_{0}$ phase $(\mathrm{CoPt})$ and the soft magnetic $\mathrm{L}_{2}$ phase $\left(\mathrm{CoPt}_{3}\right)$ interleaved on the nanoscale. The resulting periodic nanocomposite exhibits exchange coupling between its hard and soft ferromagnetic constituents, thus suggesting a potential for high magnetic energy storage. The magnetic response of Co-Pt nanochessboards has, surprisingly, not been characterized so far. This investigation focuses on establishing and understanding interrelationships between processing variables and the resulting microstructure, magnetic properties in near-eutectoid Co-Pt alloys. The effects of thermal processing were characterized using X-Ray Diffraction (XRD), Vibrating Sample Magnetometry (VSM) and Transmission Electron Microscopy (TEM).

Since the phases obtained from eutectoid decomposition are non-stoichiometric, we first studied the individual $\mathrm{Co}_{41.7} \mathrm{Pt}_{58.3}$ and $\mathrm{Co}_{37.6} \mathrm{Pt}_{62.4}$ compositions that lie slightly outside the two-phase coexistence region. The magnetic properties of $\mathrm{Co}_{41.7} \mathrm{Pt}_{58.3}$ were observed to vary intricately as a function of annealing time and temperature. These changes corresponded to changes in microstructural length-scales, phases present, magnetocrystalline anisotropy of the ordered phase and dominant pinning mechanisms. This alloy exhibited a maximum coercivity of $4 \mathrm{kOe}$ along with a maximum remanence to saturation ratio of 0.8 upon annealing at $600{ }^{\circ} \mathrm{C}$ for 4 days, and a microstructure showing tweed contrast on a $\sim 15 \mathrm{~nm}$ lengthscale. The peak coercivity alloy was established to be in the initial stages of phase transformation, containing fine $\mathrm{L}_{0}$ particles with low tetragonality embedded in the disordered FCC matrix. Despite the fine particles sizes, we argue for a domain wall pinning model based on gradients in the wall energy. This arises from exchange coupling to the soft FCC matrix, and demonstrates good agreement with the experimental coercivity, highlighting the contribution of nanoscale modulation to magnetic hardness. As the phase transformation continued towards completion, the microstructure evolved into the polytwinned structure associated with lower coercivity compared to the peak coercivity sample. Longer annealing at $700{ }^{\circ} \mathrm{C}$ led to the formation of the $\mathrm{L} 1_{2}$ ordered phase in addition to $\mathrm{L1}_{0}$. The $\mathrm{Co}_{37.6} \mathrm{Pt}_{62.4}$ alloy ordered exclusively to the soft $\mathrm{L1}_{2}$ phase.
\end{abstract}


Chessboard microstructure was observed in $\mathrm{Co}_{40.2} \mathrm{Pt}_{59.8}$ and $\mathrm{Co}_{38.8} \mathrm{Pt}_{61.2}$ alloys. Slow cooling a $\mathrm{Co}_{40.2} \mathrm{Pt}_{59.8}$ sample from $750{ }^{\circ} \mathrm{C}$ to $600{ }^{\circ} \mathrm{C}$ at $40{ }^{\circ} \mathrm{C} /$ day followed by aging for 1 week produced a chessboard structure associated with maximum coercivity, 2300 Oe, along with maximum remanence to saturation ratio, 0.66. An analysis of the corresponding magnetic susceptibility curve and XRD indicated the co-existence of both ordered phases as well as the disordered phase at this stage, wherein the hard $\mathrm{L}_{0}$ and soft $\mathrm{L}_{2}$ were exchange-coupled, but the untransformed FCC phase was uncoupled. This would require the residual FCC phase to be spatially segregated, either in macroscopic regions or in regions near the peripheries of the chessboard colonies. Overaging led to an apparent broadening/breakdown of the chessboard structure, but clear trends with process conditions are yet to be isolated.

Most of our two-phase samples $\left(\mathrm{L}_{0}+\mathrm{FCC}\right.$ or $\left.\mathrm{L}_{0}+\mathrm{L}_{2}\right)$ demonstrated strong evidence of exchange coupling. However, recoil analysis showed unexpectedly limited reversibility in all cases. This may be due to the dominant effect of domain wall motion in controlling the magnetization reversal. 


\section{Acknowledgements}

The National Science Foundation facilitated this project by funding it under grant DMR-1105336 and is very gratefully acknowledged.

I would like to express my heartfelt gratitude to my advisor Prof. Jerrold Floro for his invaluable support and guidance. I really appreciate his patience and encouragement. A sincere thank you to Prof. William Soffa for his most excellent advice and insights. I would also like to thank Prof. William Johnson, Prof. James Howe and Prof. Patrick Hopkins for agreeing to be on the examining committee and for their time and inputs.

I am very grateful to Mr. Richard White for his time and great help, especially with TEM; and to Mr. Peter Schare. I would like to thank Ms. Kimberly Fitzhugh-Higgins for arranging to have paper copies of this dissertation delivered to members of the examining committee. I would also like to thank Eric Vetter for his great help with the project. Many thanks to Dr. Abhay Gautam and Dr. Jing $\mathrm{Wu}$ for training me to use the TEM; to Matthew Schneider for assistance in resolving issues related to TEM; and to Jatin Amatya for his inputs on Dark Field TEM and TEM sample preparation. A sincere thank you to Dr. Rupalee Mulay for her help with arc melting, among other things; to Bohuslava McFarland and Joe Hagan for their help with the ICP-OES; to Dr. Kathleen Shugart for her help with the rolling mill and VSM; to Dr. Laura Lewis and Ana Maria Montes Arango of Northeastern University for their help with DSC; and to Dr. Katayun Barmak for useful conversations . I am grateful to Wade Jensen, Mark Hrdy and Chris Duska for being very helpful, resourceful colleagues.

My husband Himanshu was a tremendous source of inspiration and motivation and this academic milestone would have been very difficult to reach without his support- both moral and computers-related. I am deeply grateful to my parents, Mrs. Rashmi \& Mr. Kishore Gopalrao Ghatwai; sister, Prachi; in-laws, Mrs. Urmila and Mr. Nand Kishore Paliwal; Amit Acara Prabhu, Mayapriya Mataji and the UVA Bhakti Yoga Club for being so supportive and wonderful. 


\section{Table of Contents}

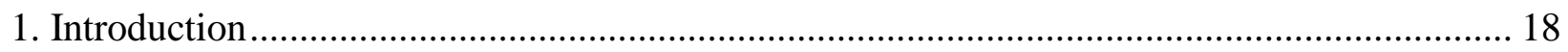

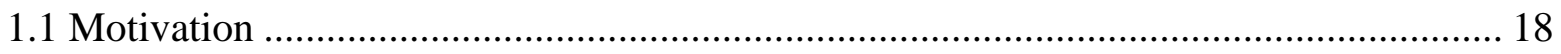

1.2 Order-disorder transformation and the Co-Pt phase diagram....................................... 19

1.2.1 Order-disorder transformation in Co-Pt and phases involved .................................. 19

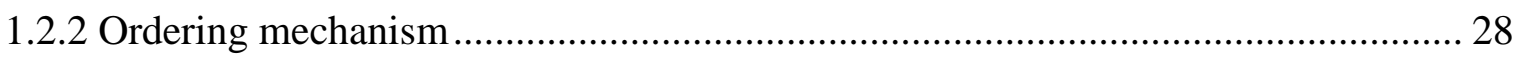

1.2.3 Eutectoid reaction and the nanochessboard structure in the Co-Pt system................ 31

1.2.4 Characterizing the presence of ordered phases ………………………………...... 34

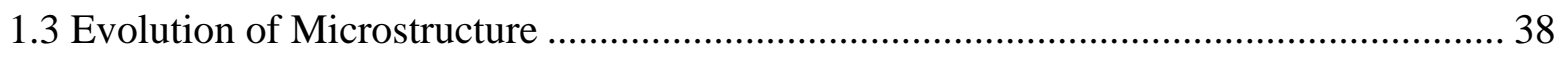

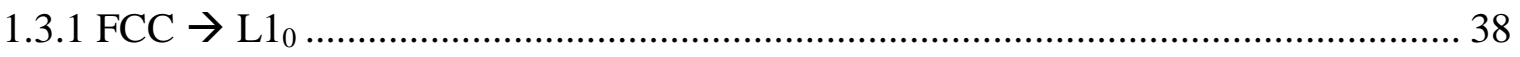

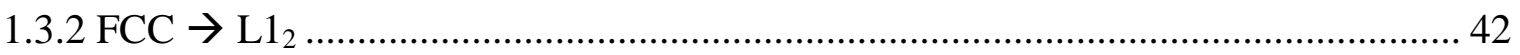

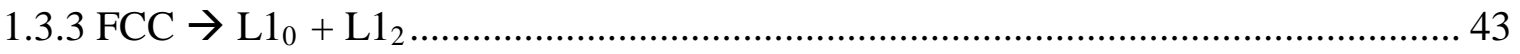

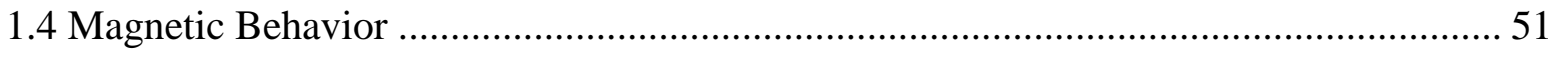

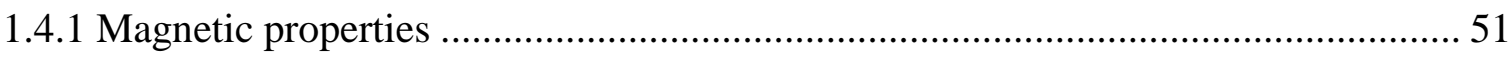

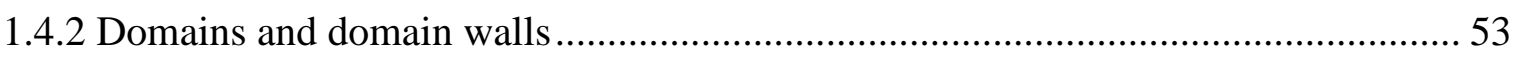

1.4.3 Magnetization and magnetization reversal .......................................................... 54

1.4.4 Exchange coupling and exchange spring behavior .................................................. 59

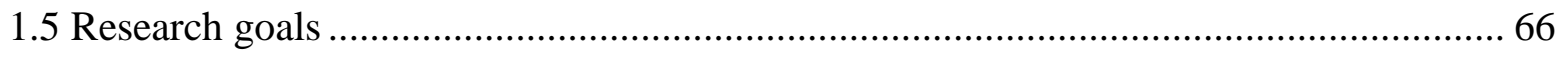

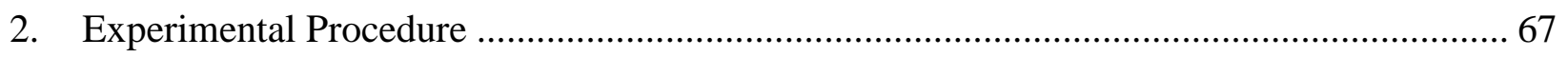

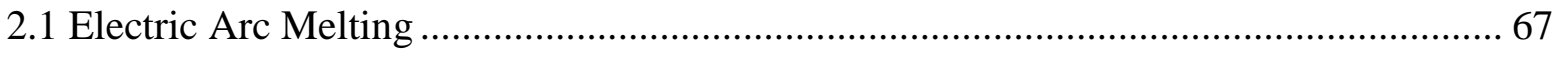

2.2 Inductively Coupled Plasma - Optical Emission Spectroscopy (ICP-OES) .................... 68

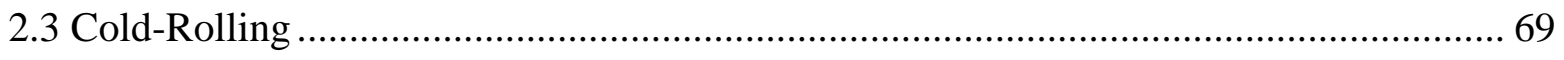

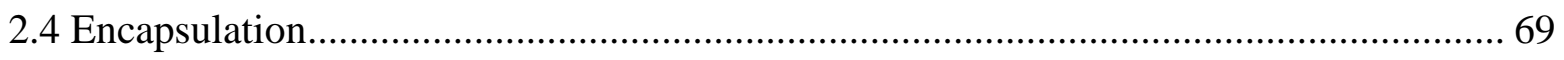

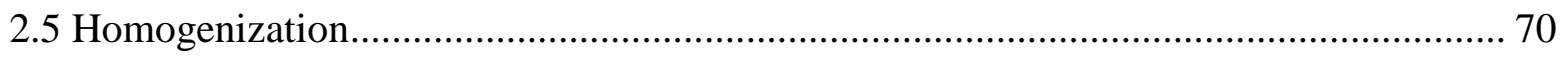

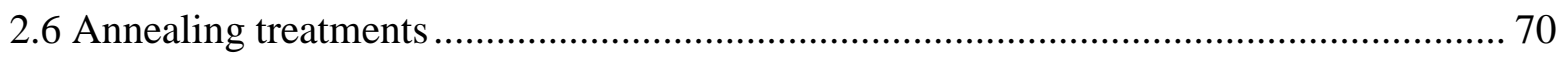




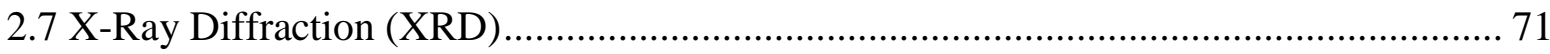

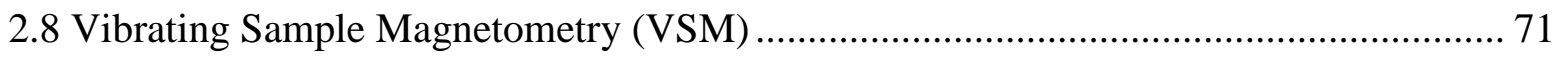

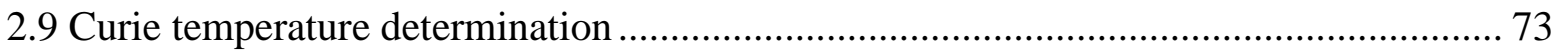

2.10 Transmission Electron Microscopy (TEM) ………………………………............. 73

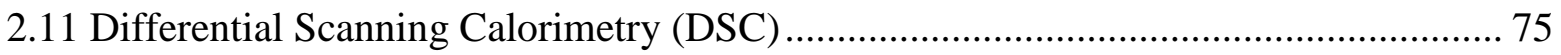

3. Compositions bordering the eutectoid isotherm: $\mathrm{Co}_{41.7} \mathrm{Pt}_{58.3}$ and $\mathrm{Co}_{37.6} \mathrm{Pt}_{62.4 \ldots \ldots \ldots \ldots \ldots \ldots \ldots \ldots . . .} 76$

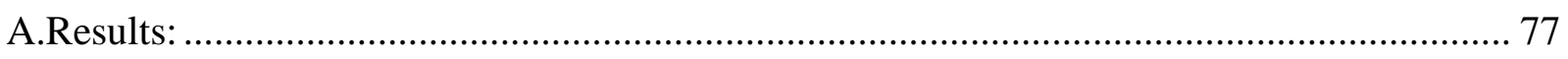

3.1 Magnetic Properties …………………………………................................................ 77

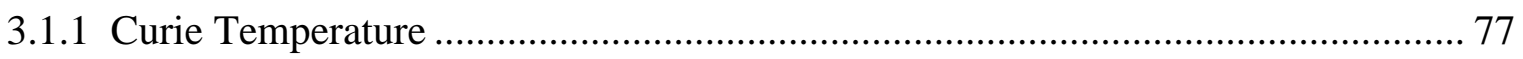

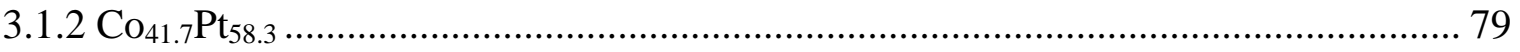

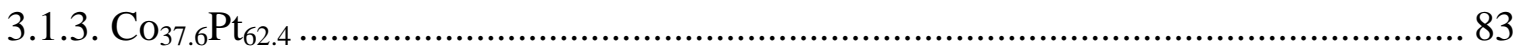

3.2 Phase Identification and Order Parameter Determination .............................................. 85

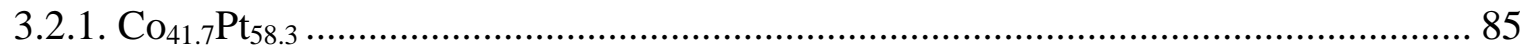

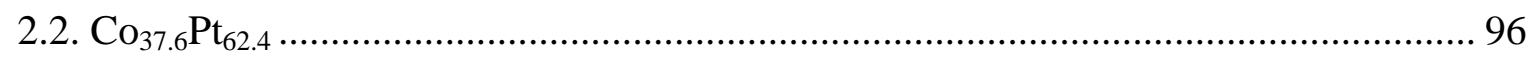

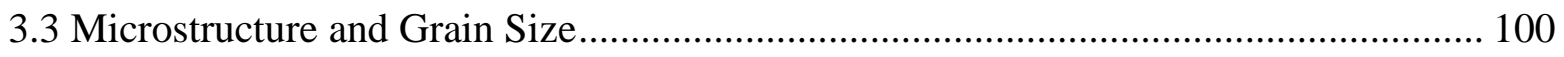

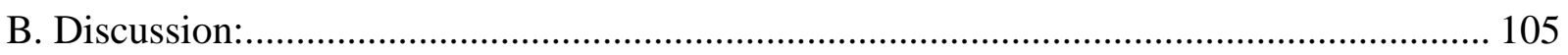

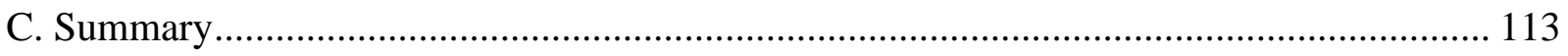

4. Compositions on the eutectoid isotherm: $\mathrm{Co}_{40.2} \mathrm{Pt}_{59.8}$ and $\mathrm{Co}_{38.8} \mathrm{Pt}_{61.2 \ldots \ldots \ldots \ldots \ldots \ldots \ldots \ldots \ldots \ldots \ldots \ldots \ldots \ldots \ldots \ldots \ldots \ldots \ldots \ldots \ldots \ldots} 114$

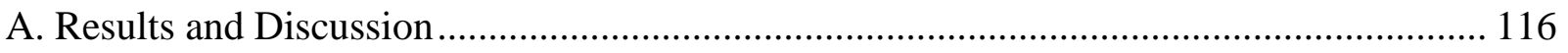

4.1 Progress of phase transformation: XRD ................................................................... 116

4.2 Accuracy of phase diagram: XRD .......................................................................... 122

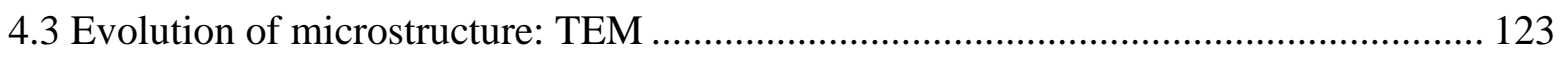

4.4 Eutectoid transformation temperature: DSC ……………………………………..... 134

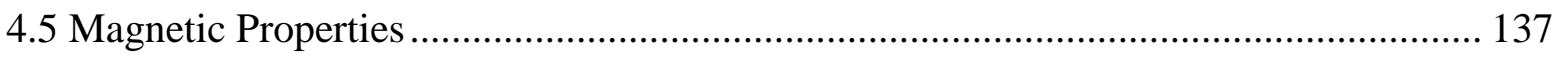

4.5.1 Effect of annealing time..................................................................................... 137 
4.5.2 Effect of Quench Temperature........................................................................ 148

4.5.3 Effect of Composition..................................................................................... 150

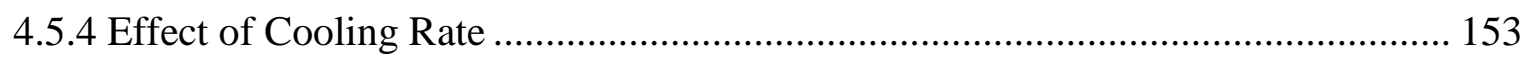

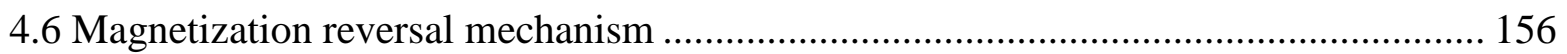

B. Summary: Processing-Structure-Property Relationships …….......................................... 161

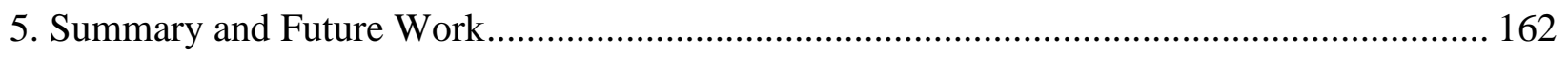

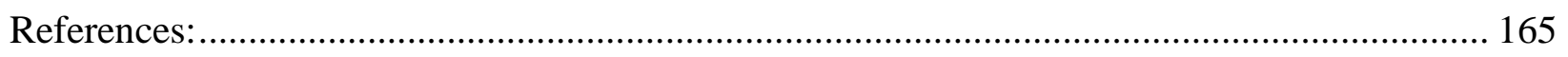

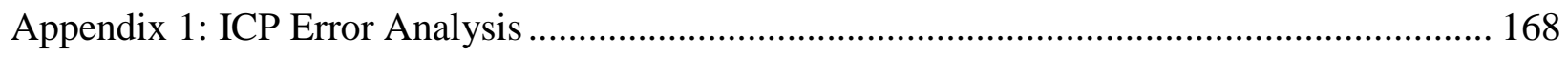

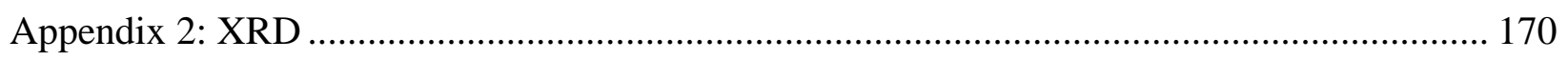

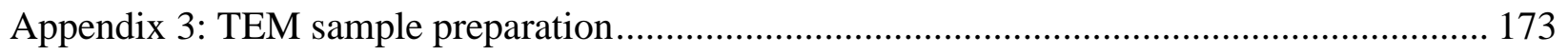

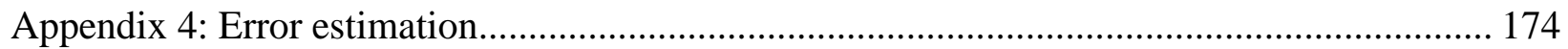

Appendix 5: Estimation of lattice strain and peak broadening .................................................. 175

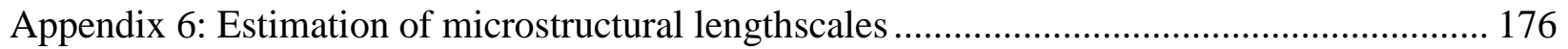

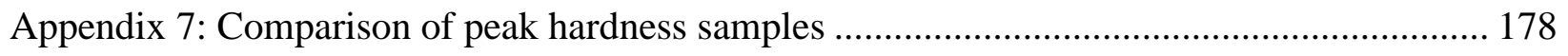

Appendix 8: Alternate processing of $\mathrm{Co}_{40.2} \mathrm{Pt}_{59.8}$ alloys ............................................................. 180 


\section{List of Figures}

Figure 1.1: Schematic representation of the nanochessboard structure where the periodic arrangement of alternate nanorods of the $\mathrm{L}_{0}$ and $\mathrm{L}_{2}$ phases gives the appearance of a

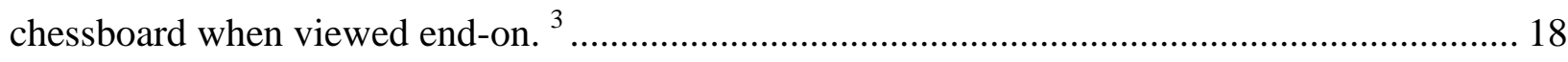

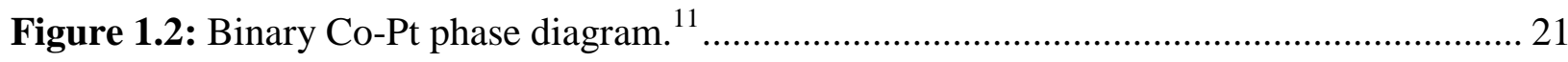

Figure 1.3: Phase diagram near the eutectoid, as determined by Leroux, et al. ${ }^{1}$ and schematic illustration of the crystal structures of phases involved. The filled dark circles represent Co atoms, the open circles represent $\mathrm{Pt}$ atoms, and the filled gray circles represent the average Co-Pt

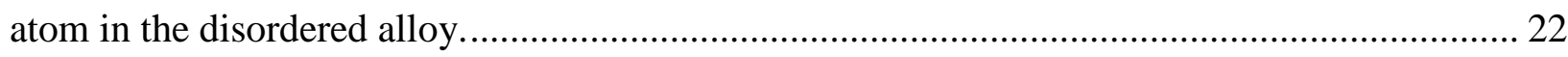

Figure 1.4: Operations (a) and (b) would respectively produce conservative and nonconservative (001) APBs in $\mathrm{Ll}_{2} \mathrm{CoPt}_{3}$. Filled circles denote $\mathrm{Co}$ and open circles denote $\mathrm{Pt}$ atoms. $^{12}$

Figure 1.5: Translation variants in $\mathrm{L}_{2}$ unit cell. Filled circles denote Co and open circles denote Pt atoms. 24

Figure 1.6: Translation variants in the $\mathrm{L} 1_{0}$ structure. 25

Figure 1.7: Orientation variants in the $\mathrm{L} 1_{0}$ structure. 26

Figure 1.8: Dependence of order parameter on the reduced temperature, $T / T_{c}$, for the case of disorder-order transformations evidencing a) first order behavior, proceeding by nucleation and growth and b) second order behavior, proceeding by a continuous change in the degree of order within the alloy. ${ }^{10}$

Figure 1.9: Schematic phase diagrams for (a) first order and (b) second/higher order disorderorder transformations. ${ }^{10}$...... 30

Figure 1.10: Free energy versus order parameter curves according to Landau's expansion for (a) first order and b) second/higher order disorder-order transformations. ${ }^{10}$ 31

Figure 1.11: (a) 110 dark field image of the nanochessboard structure in a near-eutectoid Co-Pt alloy viewed along the [001] zone axis, obtained by Leroux et al. ${ }^{1}$. The bright tiles correspond to the $\mathrm{L}_{2}$ phase while the dark tiles correspond to the $\mathrm{L} 1_{0}$ phase. (b) Schematic, idealized representation of the chessboard structure seen in (a), highlighting the crystallography of the 
constituent structural domains. ${ }^{2}$ Red arrows represent the c-axis directions of $\mathrm{L}_{0}$. (c) Actual arrangement of tile geometry.

Figure 1.12: Nanochessboard structure with tiling of ferromagnetic soft $\mathrm{L} 1_{2}$ and hard $\mathrm{L} 1_{0}$ phases, on a $20 \mathrm{~nm}$ lengthscale, obtained by Le Bouar et al. ${ }^{2}$ Yellow arrows represent the c-axis directions of $\mathrm{L} 1$

Figure 1.13: (a) Schematic [001] SADP corresponding to a region containing all three orientations of the $\mathrm{L} 1_{0}$ phase or containing the $\mathrm{L} 1_{2}$ phase. (b) [001] SADP and (c) schematic [001] SADP corresponding to the chessboard microstructure.

Figure 1.14: Tweed contrast in Fe-Pt alloy. ${ }^{23}$

Figure1.15: Schematic diagram showing the alignment, distribution of the $\mathrm{L} 1_{0}$ precipitates on alternating $\{110\}$ bands. The dark and bright particles have mutually perpendicular $\mathrm{c}$ axes, $\mathrm{c}_{1}$ and $c_{2}$ and lie on alternating regularly spaced bands; $a$ and $b$ are two possible configurations of the $\{110\}$ bands on which these particles are distributed. Note that the black and white bands are only meant to indicate the regions over which particles will coalesce. ${ }^{23,24}$

Figure 1.16: Schematic diagram depicting the atomic rearrangement in microtwins and strain free microtwin boundaries within a macrotwin. $\mathrm{C}$ axes are misoriented by $88^{\circ}$ across the microtwin boundary. ${ }^{26}$ 40

Figure 1.17: Schematic representation of the polytwinned crystal, where the colors white, gray and black are used to represent the three orientation variants of the ordered $\mathrm{L} 1_{0}$ phase. $^{27}$

Figure 1.18: Polytwinned structure in $\mathrm{L}_{0}$ alloys: (a) $100 \mathrm{DF}$ image showing microtwins in $\mathrm{CoPt}^{1}$, (b) BF image showing microtwins in FePd; profusion of APBs may be noted and (c) two macrotwins/polytwinned plates containing microtwins in FePd. ${ }^{23}$

Figure 1.19: (a) $110 \mathrm{DF}$, (b) $010 \mathrm{DF}$ and (c) $100 \mathrm{DF}$ images of $\mathrm{CoPt}_{3}$ oriented along [001], showing characteristic $\mathrm{L}_{2}$ microstructure containing APBs. ${ }^{1}$

Figure 1.20: Stages of microstructural development in the formation of the nanochessboard structure based on (a),(d),(f) experimental results and (b),(e),(f) simulation results. ${ }^{2}$ (a),(d) reveal tweed contrast, (b),(e) reveal irregular $\mathrm{L} 1_{0}$ domains and (c),(f) reveal the chessboard structure. 44 Figure 1.21: Schematic plot showing the variation of free energy with composition for coherent cubic, tetragonal phases, at a temperature $\mathrm{T}^{28}$ 
Figure 1.22: 3-D modeling of microstructure evolution involving disorder $\rightarrow$ order transformation for $(\mathrm{A}) \mathrm{c}^{\prime}<\mathrm{c}_{\mathrm{o}},(\mathrm{B}) \mathrm{c}^{\prime}>\mathrm{c}_{\mathrm{o}}$ and initiated by ordering and $(\mathrm{C}) \mathrm{c}^{\prime}>\mathrm{c}_{\mathrm{o}}$ and initiated by displacive transformation. In the upper (i.e., color) images, black represents the cubic phase while yellow, green and red respectively represent the $\mathrm{X}, \mathrm{Y}$ and $\mathrm{Z}$ variants of the tetragonal phase. The upper images in (A), (B), C) were processed to reveal more information obscured by

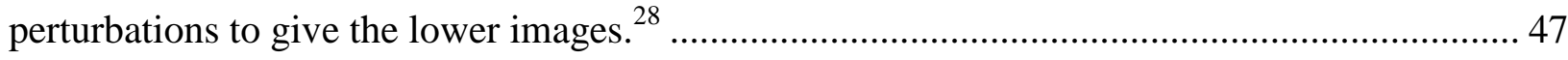

Figure 1.23: Schematic free energy curves for an alloy undergoing the eutectoid transformation.

Figure 1.24: Schematic M-H hysteresis loop. ${ }^{30}$ 51

Figure 1.25: Initial magnetization, demagnetization curves for a permanent magnet and the operating point $\mathrm{P}^{31}$

Figure 1.26: (a) Demagnetization curve and (b) variation of the energy product based on location of the operating point. ${ }^{31}$ 53

Figure 1.27: Domain formation in a ferromagnetic material leading to a reduction in the magnetostatic free energy. ${ }^{33}$ 54

Figure 1.28: Interaction between a domain wall and an inclusion. ${ }^{31}$ 56

Figure 1.29: (a) Fresnel underfocus image revealing microtwins, macrotwins and domain walls in Fe-Pd. ${ }^{30}$ (b) Schematic representation of (a). ${ }^{19}$. 57

Figure 1.30: (a) Magnetization mechanism and (b) $\mathrm{M}-\mathrm{H}$ behavior when applied external field is parallel to an easy direction. (c) Magnetization mechanism and (d) M-H behavior when applied external field is along a hard direction.

Figure 1.31: Schematic representation of hysteresis loops of single phase hard, soft magnets and an exchange-coupled nanocomposite of both these phases. ${ }^{37}$ 61

Figure 1.32: Schematic demagnetization curves in (a) exchanged coupled magnet with optimum microstructure resulting in superior magnetic properties compared to an (b) exchange coupled magnet with overaged microstructure; (c) a conventional single phase ferromagnet and (d) mixture of soft and hard ferromagnetic phases which switch independently. ${ }^{38}$ 
Figure 1.33: 1-D representation of the stages of magnetization reversal in an exchange-coupled composite showing (a) saturation remanence, (b) reversible formation of domain walls in soft mphase, (c) reversible compression of these domain walls towards the hard k-phase and (d) irreversible rotation in both phases. ${ }^{38}$ 64

Figure 1.34: Schematic representation of the optimum microstructure in an exchange coupled magnet. ${ }^{38}$

Figure 1.35: Exchange coupling, exchange spring behavior in a hard/soft nanocomposite as a function of soft phase dimension.

Figure 2.1: Binary Co-Pt alloy compositions under consideration in this study: (1) $\mathrm{Co}_{41.7} \mathrm{Pt}_{58.3 \text {, }}$

(2) $\mathrm{Co}_{37.6} \mathrm{Pt}_{62.4}(3) \mathrm{Co}_{40.5} \mathrm{Pt}_{59.5}$ and (4) $\mathrm{Co}_{38.5} \mathrm{Pt}_{61.2}$

Figure 2.2: Schematic representation of the VSM set-up. ${ }^{43}$ 72

Figure 2.3: BF image from an early TEM sample evidencing ion milling damage as a consequence of high angle milling using top and bottom milling angles of $10^{\circ}$. Such damage was prevented in subsequent samples by using shallow milling angles. 74

Figure 3.1: Phase diagram near the eutectoid, as determined by Leroux, et al. ${ }^{1}$. The vertical dashed lines show the compositions under consideration in this chapter. The horizontal lines indicate the isothermal aging temperatures that were studied in detail. 76

Figure 3.2: Curie temperatures for the two most highly transformed samples 78

Figure 3.3: Variation of magnetic properties (a) Coercivity, (b) remanence and (c) saturation magnetization and (d) BHmax, as a function of aging time and temperature. Red circles indicate an annealing temperature of $700{ }^{\circ} \mathrm{C}$ while gray squares denote $600{ }^{\circ} \mathrm{C}$. Lines through the data are intended only as guides to the eye. 81

Figure 3.4: Remanence ratio vs. aging time at $600{ }^{\circ} \mathrm{C}$ (blue squares) and $700{ }^{\circ} \mathrm{C}$ (red circles). 82 Figure 3.5: (a) A comparison of the $\mathrm{M}-\mathrm{H}$ hysteresis loops comparing the base material with the peak hardness samples annealed at $600{ }^{\circ} \mathrm{C}$ for 96 hours and $700{ }^{\circ} \mathrm{C}$ for 2.5 hours, for the $\mathrm{Co}_{41.7} \mathrm{Pt}_{58.3}$ alloy. (b) An enlarged view highlighting the initial magnetization behavior for these samples. The dashed lines indicate the corresponding coercivities. 83 
Figure 3.6: XRD spectra from the $\mathrm{Co}_{41.7} \mathrm{Pt}_{58.3}$ alloy for (a) base material, and samples annealed at (b) $600{ }^{\circ} \mathrm{C}$ for 96 hours, (c) $600{ }^{\circ} \mathrm{C}$ for 720 hours, (d) $700{ }^{\circ} \mathrm{C}$ for 2.5 hours, and (e) $700{ }^{\circ} \mathrm{C}$ for 504 hours

Figure 3.7: (a) (001) and (b) \{200\}/ (002) X-ray diffraction peaks from the annealing treatment at $700{ }^{\circ} \mathrm{C}$ for 504 hours. (c) (001) and (d) $\{200\} /(002)$ peaks from the annealing treatment at $600{ }^{\circ} \mathrm{C}$ for 720 hours. Based on appropriate peak fitting, the (001) peak actually reflected contributions from two components: $(001)_{\mathrm{L} 10}$ and $\{100\}_{\mathrm{L} 12}$, occurring at slightly different values of $2 \square$ 88

Figure 3.8: $X R D$ spectra from the $\mathrm{Co}_{37.6} \mathrm{Pt}_{62.4}$ alloy: (a) the base material; samples annealed at (b) $600{ }^{\circ} \mathrm{C}$ for $310 \mathrm{hr}$., and (c) $700{ }^{\circ} \mathrm{C}$ for $233 \mathrm{hr}$. 97

Figure 3.9: (a-c) Bright Field and (d,e) Dark Field (bottom row) and (f) Dirty Dark Field TEM micrographs for $\mathrm{Co}_{41.7} \mathrm{Pt}_{58.3}$ samples annealed at (a,d) $600{ }^{\circ} \mathrm{C}$ for 96 hours; (b,e) $600{ }^{\circ} \mathrm{C}$, for 720 hours; and (c,f) $700{ }^{\circ} \mathrm{C}$ for 24 hours. All images are along the $<001>$ zone axis except for (c), which is along the $\langle 310\rangle$ zone axis. The Bright Field images are all on the same length scale to facilitate a direct comparison.Corresponding crystal directions and $\mathbf{g}$ vectors are shown. 101

Figure 3.10: Schematic microstructure in $\mathrm{Co}_{41.7} \mathrm{Pt}_{58.3}$ sample annealed at $600{ }^{\circ} \mathrm{C}$ for 720 hours.

Figure 3.11: (a) Selected area diffraction pattern along the [001] zone axis for the peak hardness sample annealed at $600{ }^{\circ} \mathrm{C}$ for 96 hours. Key superlattice reflections are identified using red circles. The pointer is over the zero beam. (b) Schematic representation of the (001) diffraction pattern.. 103

Figure 3.12: Bright field TEM image from sample which underwent the sequenced heat treatment. 105

Figure 3.13: $\mathrm{L} 1_{0}$ nanoparticles (circles) embedded in a softer $\mathrm{A} 1$ matrix, showing a domain wall and its internal spin configurations. The domain wall advances by movement of the Néel kink to the new position shown with dashed lines, thus reversing the particle (and nearby matrix) shown in gray.

Figure 4.1: The processing space for continuously cooled samples from $\mathrm{Co}_{40.2} \mathrm{Pt}_{59.8}$ and $\mathrm{Co}_{38.8} \mathrm{Pt}_{61.2}$ alloys. 
Figure 4.2: $\{001\},\{200\} /(002),\{311\} /(113) \mathrm{XRD}$ peaks from $\mathrm{Co}_{40.2} \mathrm{Pt}_{59.8}$ samples continuously cooled from 750 to $600{ }^{\circ} \mathrm{C} @ 40{ }^{\circ} \mathrm{C} /$ day, as a function of annealing time at $600{ }^{\circ} \mathrm{C}$. 116

Figure 4.3: $\{001\},\{200\} /(002),\{311\} /(113)$ XRD peaks from $\mathrm{Co}_{40.2} \mathrm{Pt}_{59.8}$ and $\mathrm{Co}_{38.8} \mathrm{Pt}_{61.2}$ samples continuously cooled from 750 to $650{ }^{\circ} \mathrm{C} @ 40{ }^{\circ} \mathrm{C} /$ day and then water quenched....... 122

Figure 4.4: 110 DF images comparing (a) $\mathrm{Co}_{40.2} \mathrm{Pt}_{59.8}$ and (b) $\mathrm{Co}_{38.8} \mathrm{Pt}_{61.2}$ samples, both continuously cooled from 750 to $650{ }^{\circ} \mathrm{C} @ 40{ }^{\circ} \mathrm{C} /$ day and then water quenched. 125

Figure 4.5: An overview of $110 \mathrm{DF}$ images from $\mathrm{Co}_{40.2} \mathrm{Pt}_{59.8}$ samples continuously cooled from 750 to $600{ }^{\circ} \mathrm{C} @ 40{ }^{\circ} \mathrm{C} /$ day and then annealed for 0,1 and 2 weeks at $600{ }^{\circ} \mathrm{C}$. 126

Figure 4.6: (a) 110 DF of well-developed chessboard in $\mathrm{Co}_{40.2} \mathrm{Pt}_{59.8}$ sample continuously cooled from 750 to $600{ }^{\circ} \mathrm{C} @ 40{ }^{\circ} \mathrm{C} /$ day followed by water quenching. This is a higher magnification view of the chessboard on the lower right hand corner in Figure 4.6. (b) [001] SADP from the region imaged in (a). $R$.

Figure 4.7: (a) $110 \mathrm{DF}$ of well-developed chessboard from another region in the $\mathrm{Co}_{40.2} \mathrm{Pt}_{59.8}$ sample continuously cooled from 750 to $600{ }^{\circ} \mathrm{C} @ 40{ }^{\circ} \mathrm{C} /$ day followed by annealing at $600{ }^{\circ} \mathrm{C}$ for 1 week. The blue arrow points to an $\mathrm{L}_{0}$ plate while the red arrow points to a region with altered tile geometry, separating colonies of chessboards. (b) [001] SADP from the region imaged in (a).....

Figure 4.8: (a) 110 DF of well-developed chessboard from another region in the $\mathrm{Co}_{40.2} \mathrm{Pt}_{59.8}$ sample continuously cooled from 750 to $600{ }^{\circ} \mathrm{C} @ 40{ }^{\circ} \mathrm{C} /$ day followed by annealing at $600{ }^{\circ} \mathrm{C}$ for 2 weeks. (b) [001] SADP from the region imaged in (a).

Figure 4.9: $110 \mathrm{DF}$ images $\mathrm{Co}_{40.2} \mathrm{Pt}_{59.8}$ sample continuously cooled from 750 to $600{ }^{\circ} \mathrm{C} @ 40$ ${ }^{\circ} \mathrm{C} /$ day followed by water quenzching highlighting $(\mathrm{a}, \mathrm{b})$ band-shaped chessboard colonies indicated by green arrows and (b) APBs indicated by red arrows.

Figure 4.10: (a) 010, 100 and $110 \mathrm{DF}$ images of band-shaped colonies in $\mathrm{Co}_{40.2} \mathrm{Pt}_{59.8}$ samples continuously cooled to $600{ }^{\circ} \mathrm{C}$ and annealed for 2 weeks (b) [001] SADP from the region imaged in (a).

Figure 4.11: Heat flow versus temperature plots from two DSC cooling experiments performed on a $\mathrm{Co}_{40.2} \mathrm{Pt}_{59.8}$ sample originally processed via slow cooling to $650{ }^{\circ} \mathrm{C}$, followed by water quenching. 
Figure 4.12: Variation of magnetic properties with annealing time at $600{ }^{\circ} \mathrm{C}$ in continuously cooled $\mathrm{Co}_{40.2} \mathrm{Pt}_{59.8}$ 'chessboard' samples. The dashed lines are guides to the eye. 138

Figure 4.13: Initial magnetization curve for $\mathrm{Co}_{40.2} \mathrm{Pt}_{59.8}$ sample continuously cooled from 750 to $600{ }^{\circ} \mathrm{C}$ and then held at $600{ }^{\circ} \mathrm{C}$ for 1 week, resulting in maximum coercivity. 139

Figure 4.14: Major $\mathrm{M}-\mathrm{H}$ loops in $\mathrm{Co}_{40.2} \mathrm{Pt}_{59.8}$ samples continuously cooled to $600{ }^{\circ} \mathrm{C}$ and annealed for 0, 1 or 2 weeks. Minor "recoil" loops are also shown. The coercivity and remanence ratio for each sample is also summarized.

Figure 4.15: Susceptibilities obtained during magnetization reversal from positive to negative saturation, corresponding to major M-H loops shown in Figure 4.14. 141

Figure 4.16: Comparing (A) the deduced $\mathrm{M}-\mathrm{H}$ loop from a coupled but overaged sample as published by Kneller and Hawig ${ }^{38}$ (B) the $\mathrm{M}-\mathrm{H}$ loop from our $\mathrm{Co}_{40.2} \mathrm{Pt}_{59.8}$ sample annealed for 2 weeks.

Figure 4.17: Enlarged view of relevant section from Figure 4.14, with an emphasis on the recoil loops. 143

Figure 4.18: A schematic demagnetization curve and recoil loop. 144

Figure 4.19: (A) The recoverable remanence $M_{r e c} / M_{r}$ and (B) The recoverable saturation $M_{\text {rec }}$ / $\mathrm{M}_{\mathrm{s}}$

Figure 4.20: Schematic illustration of a lateral domain wall responsible for lack of exchange spring behavior in an exchange coupled nanocomposites. 146

Figure 4.21: $\mathrm{M}-\mathrm{H}$ hysteresis loops and susceptibility versus field curves from $\mathrm{Co}_{40.2} \mathrm{Pt}_{59.8}$ samples continuously cooled at the rate of $40{ }^{\circ} \mathrm{C} /$ day, from 750 to 690,650 and $600{ }^{\circ} \mathrm{C}$ respectively, and then water quenched.

Figure 4.22: $\mathrm{M}(\mathrm{H})$ as a function of composition in samples continuously cooled to $650{ }^{\circ} \mathrm{C}$ and annealed for 3.5 days at that temperature. 150

Figure 4.23: Variation of magnetic properties with annealing time in $\mathrm{Co}_{40.2} \mathrm{Pt}_{59.8}$ and $\mathrm{Co}_{38.8} \mathrm{Pt}_{61.2}$ samples upon slow cooling from 750 to $650{ }^{\circ} \mathrm{C}$, with a cooling rate of $40{ }^{\circ} \mathrm{C} /$ day. 151

Figure 4.24: Magnetic properties as a function of quench temperature in $\mathrm{Co}_{40.2} \mathrm{Pt}_{59.8}$ and $\mathrm{Co}_{38.8} \mathrm{Pt}_{61.2}$ samples obtained upon cooling from $750{ }^{\circ} \mathrm{C}$ at $40{ }^{\circ} \mathrm{C} /$ day. Saturation magnetization 
values of the disordered, single-phase base materials are also indicated in the $\mathrm{M}_{\mathrm{s}}$ versus quench temperature plot.

Figure 4.25 (A) Variation of magnetic properties with time and cooling rate in $\mathrm{Co}_{40.2} \mathrm{Pt}_{59.8}$, for annealing at $650^{\circ} \mathrm{C}$. (B) Variation of magnetic properties with time and cooling rate in $\mathrm{Co}_{38.8} \mathrm{Pt}_{61.2}$, for annealing at $650{ }^{\circ} \mathrm{C}$. 155

Figure 4.26: An example of an initial magnetization curve distinctly reflecting coherent rotation. 58

Figure 4.27: Schematic representation of a single $\mathrm{L} 1_{0}$ nanorod within the colony. 158

Figure 4.28: Schematic representation of a domain wall (red) bridging across all rods in a chessboard colony. 159

Figure 5.1: $M$ versus $H$ and susceptibility (dM/dH versus $H$ ) curves for $\mathrm{Co}_{40.2} \mathrm{Pt}_{59.8}$ samples slow cooled from 750 to $650{ }^{\circ} \mathrm{C}$, as a function of cooling rate. 164

Figure A2.1: (a) 2 peak Gaussian fit to (001) reflection where the peak at lower $2 \theta$ corresponds to $\mathrm{L1}_{2}$ and higher $2 \theta$ to $\mathrm{L} 1_{0}$; (b) 4 peak Gaussian fit to the $\{200\} / 002$ reflections where the two narrow peaks at lower $2 \theta$ correspond to $(200)_{\mathrm{K} \alpha 1}$ (solid curve) and to $(200)_{\mathrm{K} \alpha 2}$ (dashed curve) from the soft phases: $\mathrm{A} 1+\mathrm{L1}_{2}$; the broad peak at lower $2 \theta$ corresponds to (200) from $\mathrm{L} 1_{0}$ and the broad peak at higher $2 \theta$ corresponds to (002) from $\mathrm{L1}_{0}$. 172

Figure A6.1: Micrographs used for estimating microstructural lengthscale for sample annealed at $600{ }^{\circ} \mathrm{C}$ for 720 hours. 176

Figure A6.2: Micrographs used for estimating microstructural lengthscale for sample annealed at $600{ }^{\circ} \mathrm{C}$ for 96 hours.

Figure A6.3: Micrograph used for estimating microstructural lengthscale for sample annealed at $700{ }^{\circ} \mathrm{C}$ for 24 hours. 177

Figure A7.1: $\mathrm{M}-\mathrm{H}$ loops of the peak hardness $\mathrm{Co}_{61.7} \mathrm{Pt}_{58.3}$ alloy, which lies outside the eutectoid isotherm, and the peak hardness $\mathrm{Co}_{40.2} \mathrm{Pt}_{59.8}$ alloy, which lies on the eutectoid isotherm and exhibited the nanochessboard structure. 178

Figure A7.2: Susceptibility curves from peak hardness (A) $\mathrm{Co}_{40.2} \mathrm{Pt}_{59.8}$ chessboard and (B) $\mathrm{Co}_{61.7} \mathrm{Pt}_{58.3}$ samples. 
Figure A8.1: Comparison of microstructures of $\mathrm{Co}_{40.2} \mathrm{Pt}_{59.8}$ samples step cooled and continuously cooled from 750 to $600{ }^{\circ} \mathrm{C}$ and then held for 2 weeks at $600{ }^{\circ} \mathrm{C}$.

\section{List of Tables}

Table 3.1: Variation of magnetic properties viz. saturation magnetization, remanent

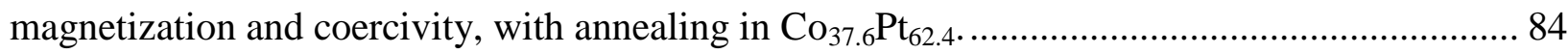

Table 3.2: Results from quantitative $x$-ray analysis of fully transformed $\mathrm{Co}_{41.7} \mathrm{Pt}_{58.3}$ sample.... 93

Table 3.3: Scherrer particle size of key $\mathrm{Co}_{41.7} \mathrm{Pt}_{58.3}$ samples.......................................... 96

Table 3.4: Lattice parameters of off-stoichiometric $\mathrm{L} 1_{0}, \mathrm{~L} 1_{2}$ phases in the vicinity of the twophase coexistence region, as well as corresponding A1 base materials. 97

Table 3.5: Microstructural lengthscales for key samples 102

Table 4.1: Volume fractions of constituent phases in $\mathrm{Co}_{40.2} \mathrm{Pt}_{59.8}$ samples continuously cooled from 750 to $600{ }^{\circ} \mathrm{C} @ 40{ }^{\circ} \mathrm{C} /$ day, as a function of annealing time at $600{ }^{\circ} \mathrm{C}$. 120 


\section{List of Symbols}

$\langle\mathbf{C o}\rangle,\langle\mathbf{P t}\rangle$ : Ppm values of elements

$\mathcal{A}$ : Area of pinning sites per unit area of domain wall

a, c: Lattice parameters

A: Exchange constant

$\mathbf{A}_{\mathbf{0}}$ : Fraction of domain wall area containing APBs

$\mathcal{B}$. Peak width at half the maximum intensity, FWHM

B: Magnetic flux density

b: Thickness of region

BH $_{\text {max }}$ : Maximum energy product

B $_{\mathbf{r}}$ : Remanent induction

$\mathbf{c}^{\prime}, \mathbf{c}_{\mathbf{o}}, \mathbf{C}$ : Composition

D: Amplitude of oscillation

d: Inter-planar spacing

e: strain

E: Energy density of domain wall

$\mathbf{E}_{\text {in: }}$ Interaction energy

F: Structure factor

f: Atomic scattering factor

G: Gibbs free energy

g: Atomic fraction of solute atoms in alloy

H: Applied external magnetic field

$\mathcal{H}$ : Enthalpy

$\mathbf{H}_{\mathbf{c}}$ : Coercivity; also used to denote $\mathrm{H}_{\mathrm{ci}}$.

$\mathbf{H}_{\mathbf{c i}}$ : Intrinsic coercivity

$\mathbf{H}_{\mathbf{d}}$ : Demagnetizing field

I: Peak intensity

J: Interaction strength

j: Mass

$\mathbf{K}, \mathbf{K}^{\prime}$ : Magnetocrystalline anisotropy constant

I : Rod length/2

LP: Lorentz Polariation factor

M: Magnetization

m: Peak multiplicity

$\mathbf{M}_{\mathbf{r}}$ : Remanence

$\mathbf{M}_{\mathbf{s}}$ : Saturation magnetization

n: Number of atoms in solution

$\mathbf{N}$ : Coupling constant

$\mathbf{N}_{\mathrm{A}}$ : Avogadro's number

P: Pressure

p: Rod diameter/2

r :Particle size

S: Entropy

$\mathbf{S}_{\mathbf{i}}, \mathbf{S}_{\mathbf{j}}:$, spins 
T: Temperature

t: Time

$\mathbf{T}_{\mathbf{c}}$ : Critical temperature above which the arrangement of atoms becomes disordered

$\mathbf{T}_{\mathbf{i}}{ }^{-}$: ordering instability temperature

U: Internal energy

$\mathbf{V}, \mathbf{V}^{\prime}, \mathbf{V}^{\prime \prime}$ : Volume

$\mathbf{V}_{\text {coil }}$ : Voltage induced in pickup coil

X: Mole fraction

z: Vertical position of sample

$\Delta \mathrm{N}$ : Difference between demagnetizing coefficients along major and minor axes

$\alpha, \beta, \tau, \mathbf{k}:$ constants

$\chi$ : susceptibility

$\delta$ : Domain wall thickness

$\varepsilon$ : Bond energy

$\phi$ : Magnetic flux

$\gamma$ : Domain wall energy

$\eta$ : Order parameter

$\lambda$ : Wavelength

$\lambda^{\prime}$ : Microstructural lengthscale

$\lambda_{\mathrm{c}}$ : Correlation length

$\Lambda$ : Scherrer particle size

$\mu$ : Magnetic moment

$v$ : Frequency of oscillation

$\theta$ : Angle

$\rho$ : Number of bonds

$\sigma$ : Short range order parameter

$\omega$ : Thickness of perturbed region

$\xi$ : Molar mass 


\section{Introduction}

\subsection{Motivation}

The 'nanochessboard' microstructure was obtained upon appropriate thermal processing in neareutectoid Co-Pt alloys containing 60 atom \% Pt by Leroux et al. ${ }^{1}$ and Le Bouar et al. ${ }^{2}$ This self-assembled structure consists of a two dimensional-periodic stacking of alternate nanorods of $\mathrm{L}_{0}$ and $\mathrm{L}_{2}$ phases separated by coherent interfaces. Viewed end-on using dark field (DF) Transmission Electron Microscopy (TEM), this structure appears as a chessboard with alternate dark and bright tiles interleaved on a $20 \mathrm{~nm}$ lengthscale; the nanorods may extend for hundreds of nanometers in the orthogonal direction. Figure 1.1 shows a schematic representation of this structure in three-dimensional space, with the dark tiles corresponding to the $\mathrm{L}_{0}$ phase and the bright tiles corresponding to the $\mathrm{L}_{2}$ phase.
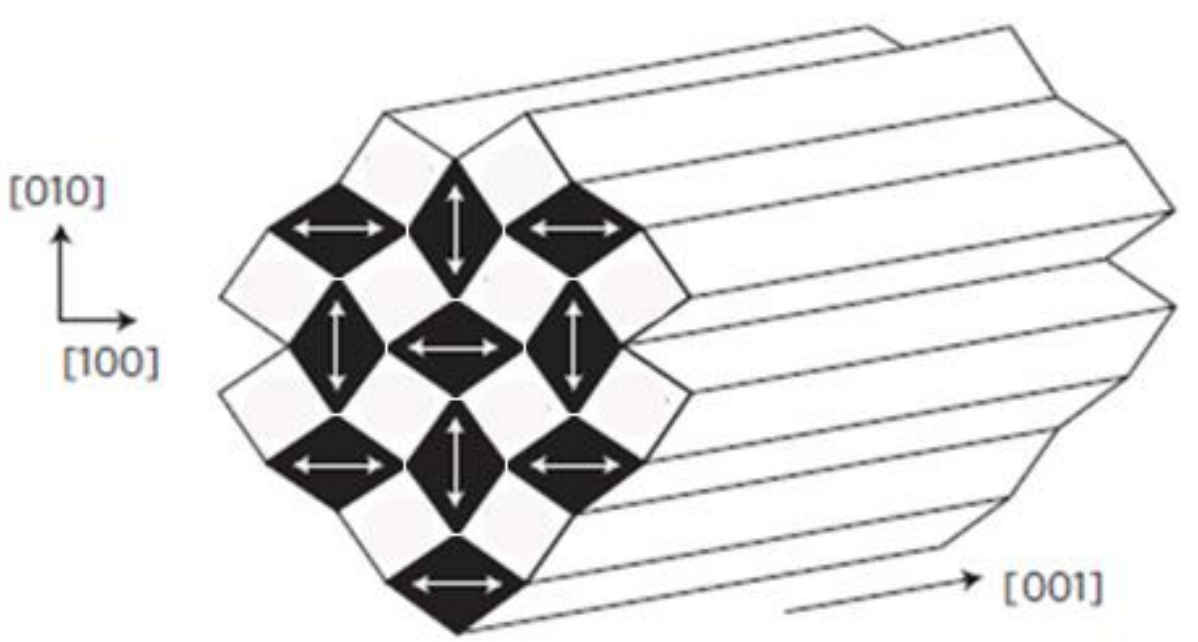

Figure 1.1: Schematic representation of the nanochessboard structure where the periodic arrangement of alternate nanorods of the $\mathrm{L}_{0}$ and $\mathrm{L1}_{2}$ phases gives the appearance of a chessboard when viewed end-on. ${ }^{3}$

The $\mathrm{L}_{0}{ }_{0}$-structured $\mathrm{CoPt}$ is a hard ferromagnet with a high uniaxial magnetocrystalline anisotropy. ${ }^{2,4}$ The $\mathrm{L1}_{2}$-structured $\mathrm{CoPt}_{3}$ phase is more isotropic and a much softer ferromagnet. The nanochessboard structure, being a highly-regular nanocomposite of these two phases, provides an ideal testbed for investigating exchange coupling between hard and soft 
ferromagnetic phases. Furthermore, the stoichiometric $\mathrm{L} 1_{2}$ phase is paramagnetic above $15{ }^{\circ} \mathrm{C}{ }^{5}$ and hence, the eutectoid Co-Pt system may prove suitable for investigating exchange coupling between hard ferromagnetic and paramagnetic phases. Exchange coupling between the two phases could also promote useful permanent magnetic properties, i.e., simultaneously high remanence and coercivity, in the nanocomposite, with the high remanence stemming from the soft ferromagnet and high coercivity from the hard ferromagnet. This should consequently enhance the stored magnetic energy, i.e., the maximum energy product $\mathrm{BH}_{\max }$, which is a figure of merit of great importance in permanent magnets. ${ }^{6} \mathrm{Co}-\mathrm{Pt}$ alloys are more ductile and chemically inert in comparison with rare earth permanent magnets. ${ }^{4}$ The processing of rare earth magnets includes magnetic alignment and sintering of fine powders which may be unsuitable for several applications. ${ }^{7}$ Rare earth magnets also oxidize easily, and chemical inhomogeneity is common at grain boundaries, making them more prone to corrosion damage. ${ }^{8}$

In spite of the potential for intriguing magnetic response, the magnetic behavior of Co-Pt nanochessboards has not been previously investigated. In this research, we have studied the interrelationships between processing, microstructure and magnetic properties in near eutectoid alloys. We have also investigated the science of exchange coupling and magnetization reversal in these alloys. The high cost of Pt would limit the scope of eutectoid Co-Pt alloys to specialty applications. However, fundamental research on the chessboard microstructure may be useful to engineer the magnetic response and provide important insights for efforts related to new materials development of ferromagnets.

\subsection{Order-disorder transformation and the Co-Pt phase diagram}

\subsubsection{Order-disorder transformation in Co-Pt and phases involved}

The equilibrium phase diagram for the binary Co-Pt system is shown in Figure 1.2. At elevated temperatures, an A1 (FCC) solid solution forms across the entire composition range. Co-Pt alloys of appropriate composition can undergo a disorder - order transformation upon cooling, or conversely, an order-disorder transformation upon heating and the phase diagram indicates the regions over which the ordered phases persist. Equiatomic CoPt transforms from the disordered A1 to the ordered $\mathrm{L1}_{0}$ phase upon cooling. The $\mathrm{L}_{0}$ phase has impressive intrinsic magnetic 
properties like a high uniaxial magnetocrystalline anisotropy approaching those of rare earth magnets $^{2,4}, 4.9 * 10^{7} \mathrm{ergs} / \mathrm{cc}$, saturation magnetization, $800 \mathrm{emu} / \mathrm{cc}$, and Curie temperature (840 K). ${ }^{9,}{ }^{10}$ Hence, most investigations of ordering and magnetism in the Co-Pt binary system have generally been based on this 1:1 composition. In comparison with $\mathrm{CoPt}, \mathrm{Fe}_{14} \mathrm{Nd}_{2} \mathrm{~B}$ rare earth magnets are associated with a magnetocrystalline anisotropy of $4.6 * 10^{7} \mathrm{ergs} / \mathrm{cc}$, saturation magnetization of $1270 \mathrm{emu} / \mathrm{cc}$ and Curie temperature of $585 \mathrm{~K} .{ }^{9} \mathrm{~A} 1: 3$ molar ratio of Co and $\mathrm{Pt}$ results in a disorder to order transformation from the disordered A1 (FCC) phase to the ordered, magnetically soft $\mathrm{L}_{2}$ phase, $\mathrm{CoPt}_{3}$.

Fig. 1.3 shows the Co-Pt phase diagram in the vicinity of the eutectoid composition. It may be noted that the coherent phase diagram around the eutectoid was determined by Leroux et $\mathrm{al}^{1}$ exclusively on the basis of TEM investigation of phase transformation of alloys in the vicinity of the eutectoid composition. It may be noted that the accuracy of the compositions used in their study was not established. Furthermore, the nanoscale phases and transformation/coherency strains inherent to the microstructures being studied will significantly affect the phase boundaries. Thus, the accuracy of the eutectoid temperature and phase boundaries are.not established; however, we will work with the available phase diagram. 


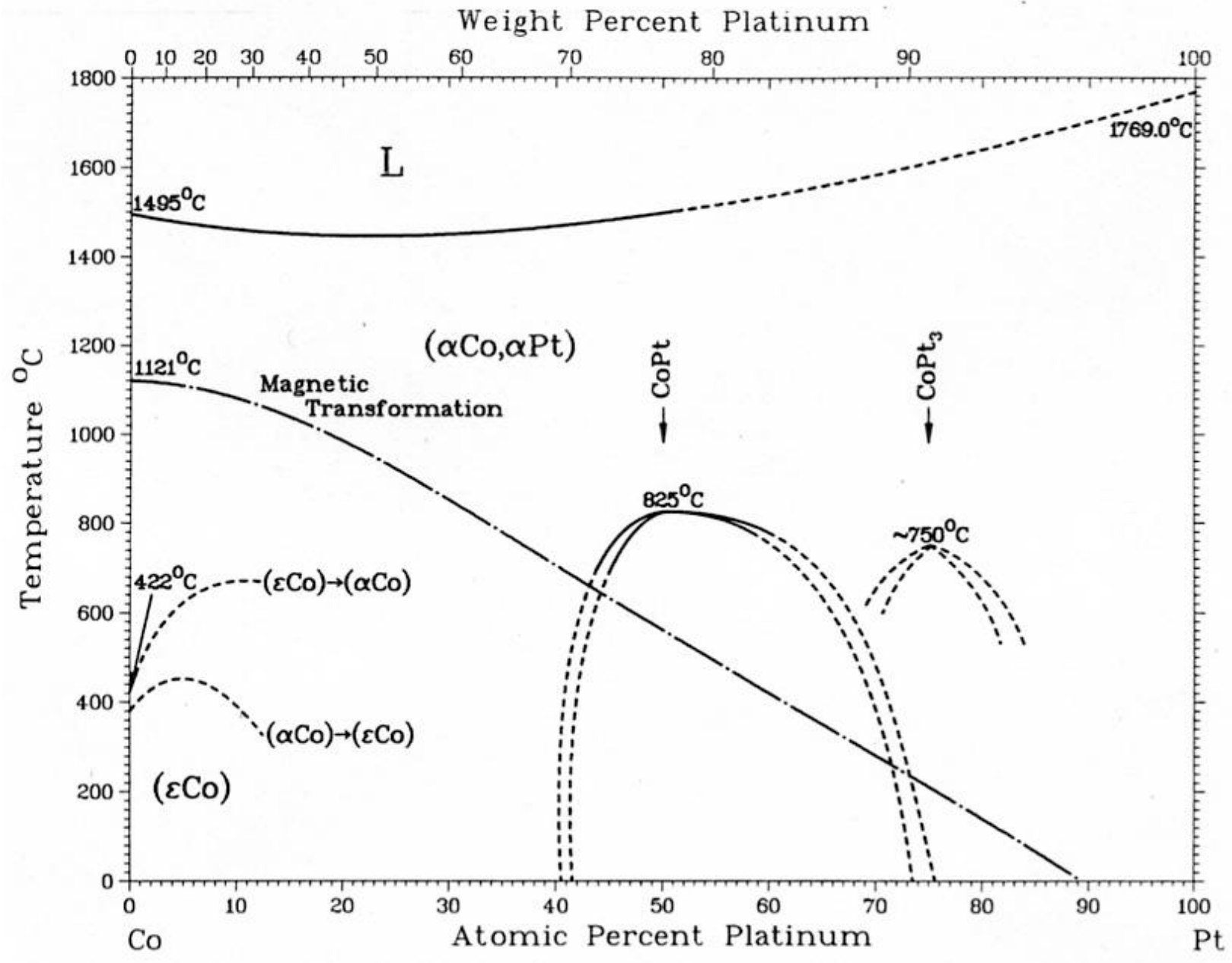

Figure 1.2: Binary Co-Pt phase diagram. ${ }^{11}$ 


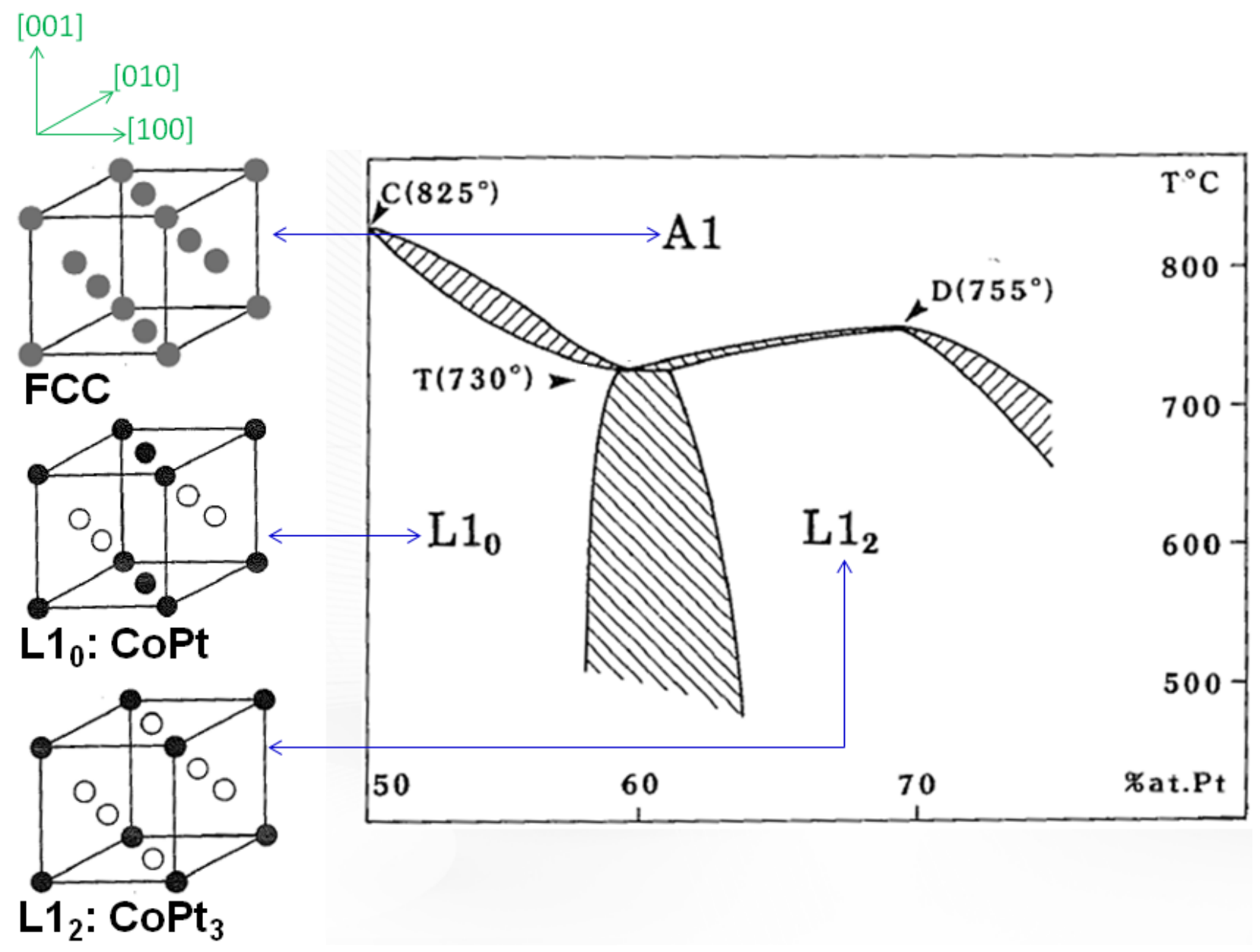

Figure 1.3: Phase diagram near the eutectoid, as determined by Leroux, et al. ${ }^{1}$ and schematic illustration of the crystal structures of phases involved. The filled dark circles represent Co atoms, the open circles represent $\mathrm{Pt}$ atoms, and the filled gray circles represent the average Co-Pt atom in the disordered alloy.

The crystal structures corresponding to the $\mathrm{A} 1, \mathrm{~L} 1_{0}$ and $\mathrm{L} 1_{2}$ phases are schematically illustrated in Figure 1.3. An average atom $\mathrm{Co}_{\mathrm{x}} \mathrm{Pt}_{1-\mathrm{x}}$ occupies all sites on corners and faces viz. (000), $(1 / 2,1 / 2$, $0),(1 / 2,0,1 / 2)$ and $(0,1 / 2,1 / 2)$ in the disordered FCC structure of a binary Co-Pt alloy of composition $\mathrm{Co}_{\mathrm{x}} \mathrm{Pt}_{1-\mathrm{x}}$. The ordered $\mathrm{L}_{2}$ and $\mathrm{L}_{0}$ structures are crystallographically derived from the FCC structure. In the ordered, stoichiometric $\mathrm{Ll}_{2}$ phase, $\mathrm{CoPt}_{3}$, the Co is located at the corner sites $(0,0,0)$ whereas the Pt atoms lie on the face centers: $(1 / 2,1 / 2,0),(1 / 2,0,1 / 2)$ and $(0,1 / 2,1 / 2)$. This structure has a total of four translation variants depending on the position of the four atoms with 
respect to these lattice sites. The interface between any two translation variants is called an anti phase boundary (APB).

APBs may be classified as conservative and non conservative. ${ }^{12}$ The translation vector correlating the two variants is in the plane of the interface for a conservative APB. For the case of an (001) APB, this corresponds to an a-type exchange between the atoms, as shown in Fig.1.4. On the other hand, the translation vector correlating the two variants has a component perpendicular to the plane of the interface for a non-conservative APB. This would correspond to a b-type exchange for an (001) APB shown in Fig. 1.4. ${ }^{13}$ As an example, an (001) APB between variants (1) and (4) in Fig.1.5 is conservative while (001) APBs between variants (1) and (2) or variants (1) and (3) are non-conservative.

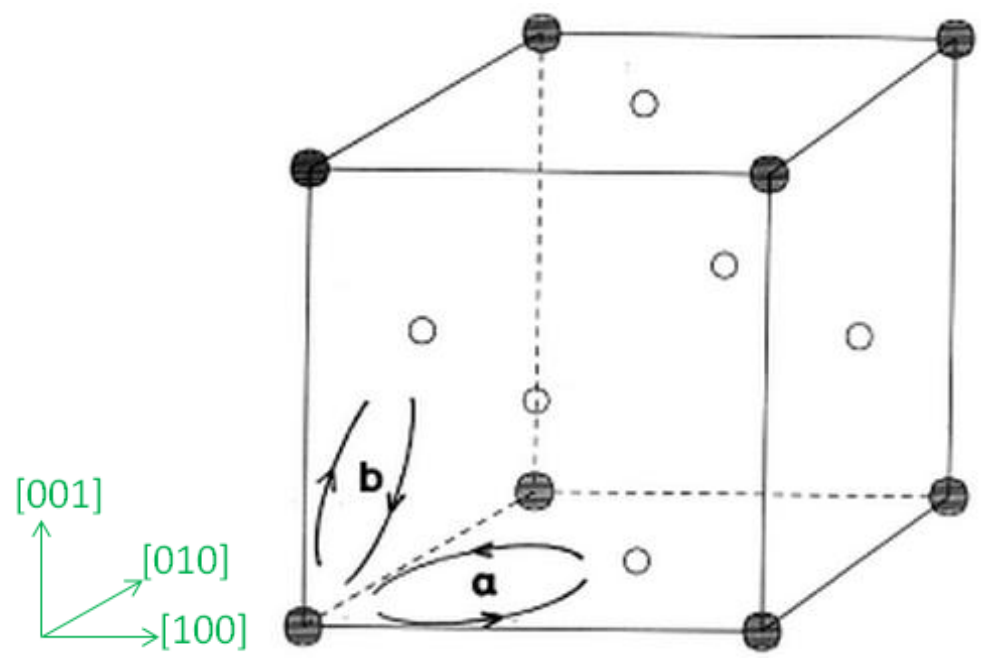

Figure 1.4: Operations (a) and (b) would respectively produce conservative and nonconservative (001) APBs in $\mathrm{L1}_{2} \mathrm{CoPt}_{3}$. Filled circles denote Co and open circles denote Pt atoms. $^{12}$ 

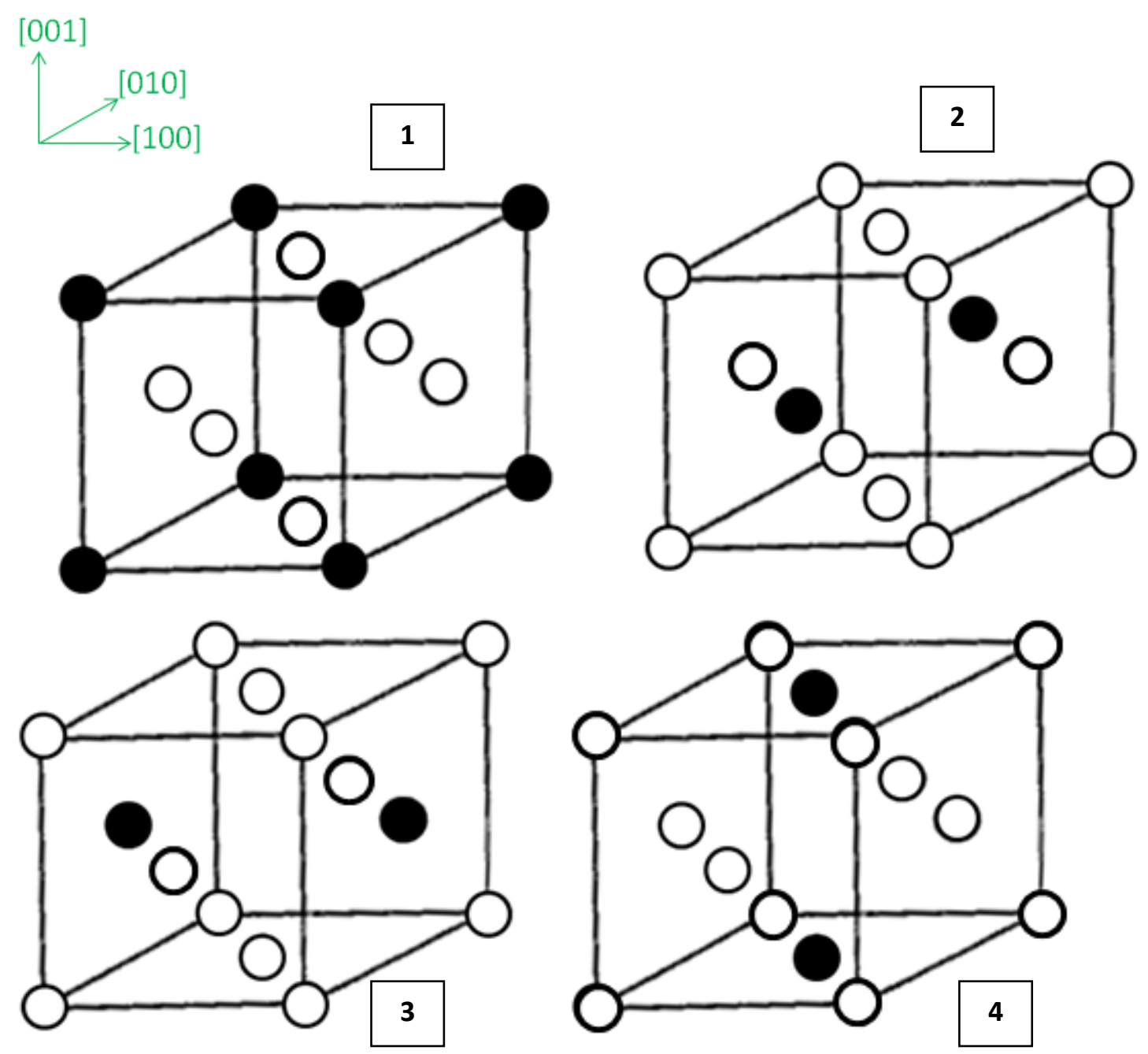

Figure 1.5: Translation variants in $\mathrm{L} 1_{2}$ unit cell. Filled circles denote Co and open circles denote Pt atoms.

In the ordered, stoichiometric $\mathrm{L}_{0}$ phase, $\mathrm{Co}$ and $\mathrm{Pt}$ atoms lie on alternate (002) planes. As shown in Figure 1.6, the $\mathrm{L} 1_{0}$ structure can have two translational variants, resulting in the presence of antiphase domains. For example, in Figures 1.3 and 1.6 (a), Co atoms are located at (000) and $(1 / 2,1 / 2,0)$ sites while Pt atoms are located at the $(1 / 2,0,1 / 2)$ and $(0,1 / 2,1 / 2)$ sites. On the other hand, in the variant shown in Figure 1.6 (b), Pt atoms are located at $(000)$ and $(1 / 2,1 / 2,0)$ sites while Co atoms are located at the $(1 / 2,0,1 / 2)$ and $(0,1 / 2,1 / 2)$ sites. The interface between the two translation variants is an $\mathrm{APB}$. Additionally, the $\mathrm{L} 1_{0}$ structure has three orientation variants depending on the orientation of the ordering half plane viz. X(100), Y (010) and Z (001), as shown in Figure 
1.7. The lattice parameter of the unit cell is 'c' along the tetragonal axis, i.e., along the normal to the ordering half plane, and ' $a$ ' along the remaining two cube axes. Orientation variants are separated by $\{101\}$ type planes. Each orientation variant has two translation variants making a total of six possible variants in this $\mathrm{L} 1_{0}$ structure. ${ }^{14}$

In equiatomic Co - Pt alloys, the highest coercivities have been reported to occur at an intermediate state of order with coherent ordered and disordered phases as opposed to completely ordered or disordered states. In fact, these properties have been reported to decay upon complete ordering. ${ }^{15}$

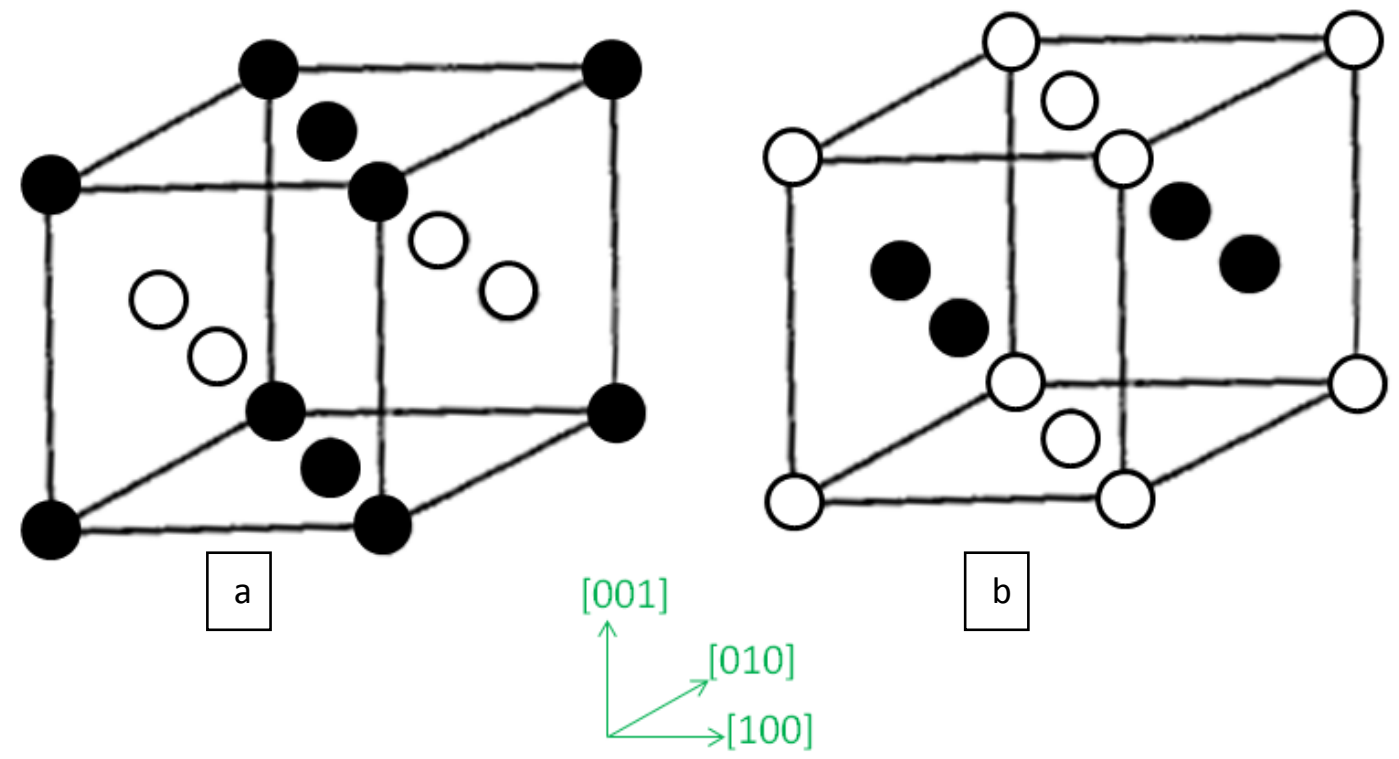

Figure 1.6: Translation variants in the $\mathrm{L} 1_{0}$ structure. 

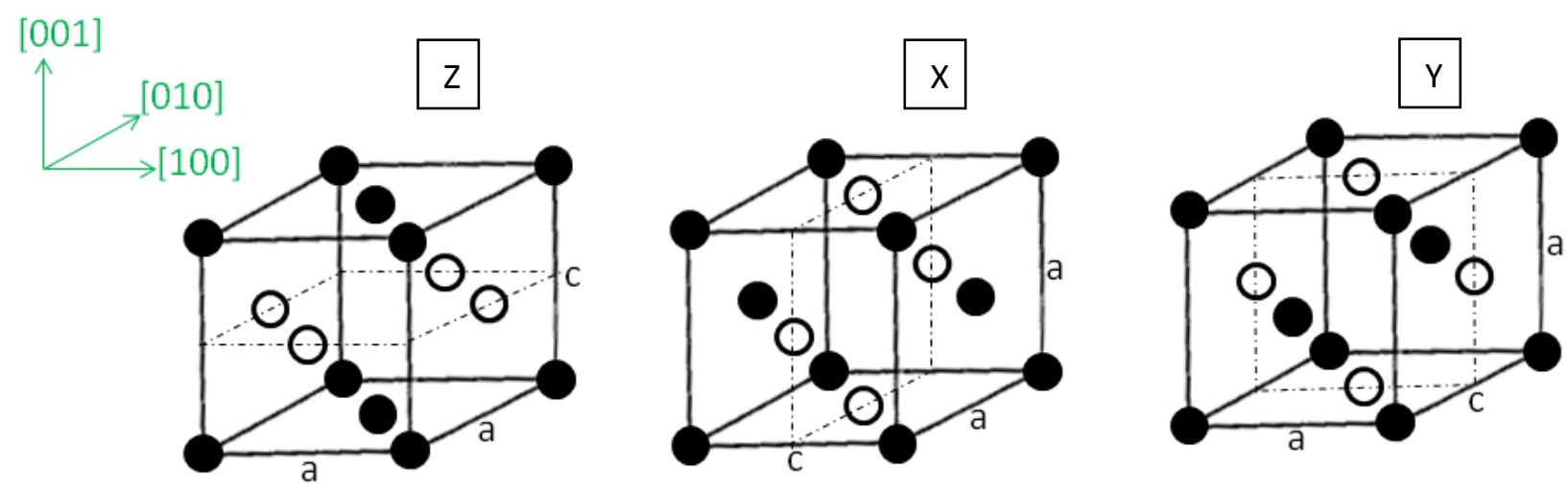

Figure 1.7: Orientation variants in the $\mathrm{L} 1_{0}$ structure.

A simple, nearest-neighbor bond-energy model can give useful insights into the ordering phenomenon. Consider a binary substitutional solid solution, containing atoms of the constituent elements $\mathrm{A}$ and $\mathrm{B}$. Let $\varepsilon_{\mathrm{AA}}, \varepsilon_{\mathrm{BB}}$ and $\varepsilon_{\mathrm{AB}}$ be the bond energies of $\mathrm{A}-\mathrm{A}, \mathrm{B}-\mathrm{B}$ and $\mathrm{A}-\mathrm{B}$ bonds respectively. The difference between the energy of an A-B bond and the average of the energies associated with the A-A and B-B bonds, $\varepsilon^{\prime}$, is given as:

$\varepsilon^{\prime}=\varepsilon_{\mathrm{AB}}-1 / 2\left(\varepsilon_{\mathrm{AA}}+\varepsilon_{\mathrm{BB}}\right)$

If $\varepsilon^{\prime}=0$, atoms of the constituent elements $\mathrm{A}$ and $\mathrm{B}$ are arranged randomly since each atom position is equivalent. Thus, in this case, the probability of a lattice site hosting an A atom will be equal to the atomic fraction of $\mathrm{A}$ atoms while the probability of a lattice site hosting a $\mathrm{B}$ atom will be equal to the atomic fraction of $\mathrm{B}$ atoms in the solid solution. This is true of the disordered Co-Pt alloys. For $\varepsilon^{\prime} \neq 0$, the atom sites stop being equivalent but are preferentially occupied. If $\varepsilon^{\prime}>0$, atoms tend to be surrounded by atoms of similar type, and tend to cluster into A-rich and B-rich groups. If $\varepsilon^{\prime}<0$, the atoms prefer to be surrounded by atoms of the opposite type, i.e., they prefer to be arranged in an ordered structure. When this is observed, it is referred to as short range order and is associated with a short range order parameter, $\sigma=\left[\rho_{\mathrm{AB}}-\rho_{\mathrm{AB}}\right.$ (random) $]$ / $\left[\rho_{\mathrm{AB}}\right.$ (max) $-\rho_{\mathrm{AB}}$ (random) $]$, where $\rho_{\mathrm{AB}}=$ observed number of bonds between dislike atoms, $\rho_{\mathrm{AB}(\text { random })}=$ number of bonds between unlike atoms in a random solution, i.e., corresponding to $\varepsilon=0$ and $\rho_{\mathrm{AB}(\max )}=$ maximum possible number of A-B bonds. ${ }^{16}$ Long range order can be found in solutions containing a simple ratio of $\mathrm{A}$ and $\mathrm{B}$ atoms where the ordered structures contain 
fewer bonds between similar neighbors. Let us consider the Co-Pt alloys undergoing transformation from the disordered $\mathrm{FCC}$ phase to the ordered $\mathrm{L}_{0}(\mathrm{CoPt})$ or $\mathrm{L}_{2}\left(\mathrm{CoPt}_{3}\right)$ phases, depending on the alloy stoichiometry. As can be seen from Figure 1.3, the disordered FCC phase contains an average atom at each lattice site and therefore, each atom is surrounded by 12 nearest neighbor atoms of the same type. In the $\mathrm{L} 1_{0}$ structure, each atom has 4 like and 8 unlike nearest neighbors. In the $\mathrm{L}_{2}$ structure, each minority atom (Co) has 12 nearest neighbors of the opposite type while each majority $(\mathrm{Pt})$ atom is surrounded by 8 like $(\mathrm{Pt})$ and 4 unlike $(\mathrm{Co})$ atoms. ${ }^{13}$

The system with the lowest free energy will be thermodynamically favored at equilibrium. The Gibbs free energy $\mathrm{G}=\mathcal{H}-\mathrm{TS}$ where $\mathcal{H}$ is the enthalpy, a measure of the heat content of system, $\mathrm{T}$ is the absolute temperature and $\mathrm{S}$ is the entropy of the system. The enthalpy is given as $\mathcal{H}=\mathrm{U}$ $+\mathrm{PV}, \mathrm{U}$ being the internal energy, the total of kinetic and potential energies possessed by all atoms comprising the system, $\mathrm{P}$ the pressure and $\mathrm{V}$ the volume. In condensed phases, $\mathcal{H} \approx \mathrm{U}$. At low temperatures, the TS term is small and thus the system is driven by the value of E, which has a negative value. For an ordering system, the lowest internal energy occurs for an arrangement of atoms corresponding to the ordered state rather than the disordered state and hence, the ordered phase exists preferentially at lower temperatures. With increasing temperature, the entropic term TS, which is higher for a disordered phase compared to an ordered phase, becomes very important and hence, the disordered phase is preferred at higher temperatures. Thus, $\mathrm{T}$ is the important factor for order-disorder transformations. ${ }^{16,} 17$ The order parameter decreases continuously upon heating until a critical temperature, $\mathrm{T}_{\mathrm{c}}$, above which the arrangement of atoms becomes disordered. This critical temperature is maximum for the ideal stoichiometric composition. Even if the composition diverges from the ideal to an extent, long range order can still be accommodated if some of the sites are vacant or occupied by atoms of the wrong type. ${ }^{16}$

The order parameter $\eta=(\mathrm{R}-\mathrm{W}) / \mathrm{R}+\mathrm{W}$, where $\mathrm{R}$ is the number of atoms occupying the correct sites and $\mathrm{W}$ is the number of atoms occupying the wrong sites, with respect to the arrangement of atoms in the ordered structure. In stoichiometric alloys, the maximum order parameter is 1 whereas in non stoichiometric alloys this is $2 \mathrm{~g}$, $\mathrm{g}$ being the atomic fraction of solute atoms in the alloy. $^{10}$ 


\subsubsection{Ordering mechanism}

According to Ehrenfest, phase transformations may be classified on the basis of the order of the reaction, i.e., the lowest differential of the free energy that exhibits discontinuity. In a first order phase transformation, the Gibbs free energy is continuous across the equilibrium transformation temperature but the first derivatives of the Gibbs free energy with respect to temperature and pressure, respectively $-\mathrm{S}$ and $\mathrm{V}$ are discontinuous. $[\partial(\mathrm{G} / \mathrm{T}) / \partial(1 / \mathrm{T})]=\mathrm{H}$ is discontinuous across the order-disorder transformation temperature, owing to the latent heat associated with the transformation. In a second order transformation, the second derivatives of the Gibbs free energy are discontinuous across the transformation temperature while the first derivatives are continuous.

Upon cooling a disordered alloy, the disorder - order transformation may proceed by one of the following two mechanisms:

1. By the nucleation and growth of the ordered domains upon overcoming the thermodynamic energy barrier for the formation of these domains. This corresponds to a first order phase transformation and occurs in $\mathrm{FCC} \rightarrow \mathrm{L} 1_{0}$ or $\mathrm{FCC} \rightarrow \mathrm{L} 1_{2}$ transformations wherein the order parameter $\eta$ drops slightly upon heating up to the critical temperature $\mathrm{T}_{\mathrm{c}}$ and then precipitously to 0 at $\mathrm{T}_{\mathrm{c}}$, as shown in Figure 1.8 (a). A steep change in order occurs at the critical temperature. Due to the sudden change in bonding environment, i.e., increase in number of like-like bonds, the disordered state has a higher internal energy and enthalpy associated with it compared to the ordered state. Thus, there is a drastic change in internal energy, enthalpy across the critical temperature. ${ }^{16}$ A schematic phase diagram for a first order disorder - order transformation is shown in Figure 1.9 (a). Herein, we may note the existence of a two-phase region where the ordered and disordered phases coexist in equilibrium. However, the thermodynamic energy barrier for nucleation of discrete nuclei of the ordered phase vanishes below the ordering instability temperature $\mathrm{T}_{\mathrm{i}}{ }^{-}$and the alloy orders continuously, thus evidencing second or higher order character, as discussed below. ${ }^{10}$

2. By a continuous change in the short-range order via local atomic rearrangement within the crystal, without an associated energy barrier, eventually resulting in long-range order. 
16 Thus, no nucleation event is required. This corresponds to a second or higher order phase transformation and occurs in $\mathrm{A} 2-\mathrm{B} 2$ transformations, such as in $\mathrm{CuZn}$. Herein, the long-range order parameter $\eta$ changes continuously, from 0 at the critical temperature $\mathrm{T}_{\mathrm{c}}$, to 1 at $0 \mathrm{~K}$, as shown in Figure 1.8 (b). No sudden change in order occurs at the critical temperature as can be seen in the figure and thus the internal energy (and enthalpy) are continuous over $\mathrm{T}_{\mathrm{c}}{ }^{16} \mathrm{~A}$ schematic phase diagram for a second/higher order disorder-order transformation is shown in Figure 1.9 (b). No two-phase region is seen in this diagram as coexistence of two compositionally- and structurally-discrete phases does not make sense in this context.

If a free-energy barrier exists for phase transformation, it will be a first order transformation. If the free-energy barrier does not exist, the phase transformation will be second-order. The product phase(s) must be crystallographic derivative(s) of the parent phase for a second order transformation to be possible. ${ }^{18}$

a)

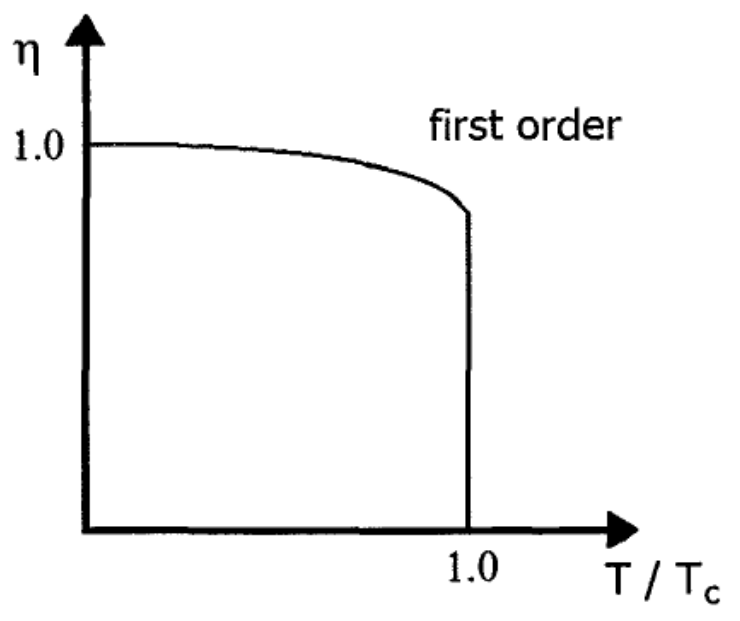

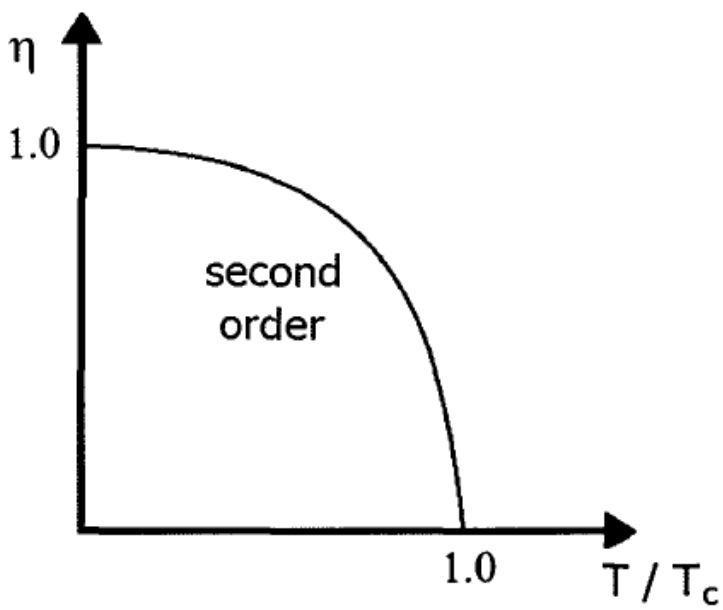

Figure 1.8: Dependence of order parameter on the reduced temperature, $T / \mathrm{T}_{c}$, for the case of disorder-order transformations evidencing a) first order behavior, proceeding by nucleation and growth and b) second order behavior, proceeding by a continuous change in the degree of order within the alloy. ${ }^{10}$ 
a)

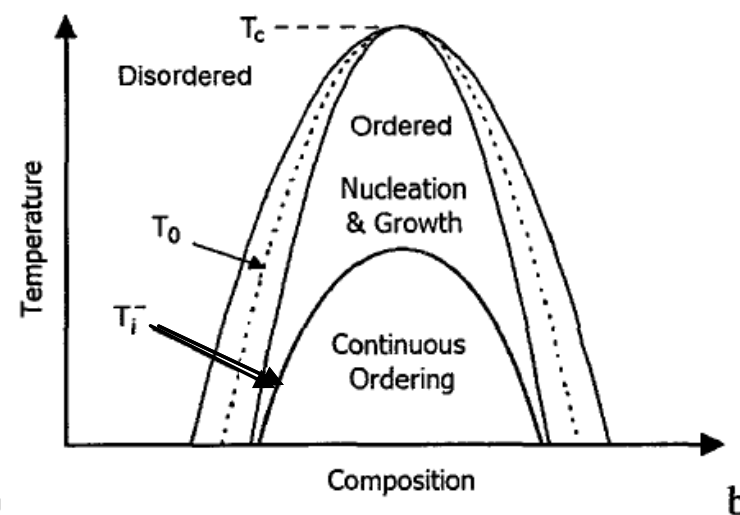

b)

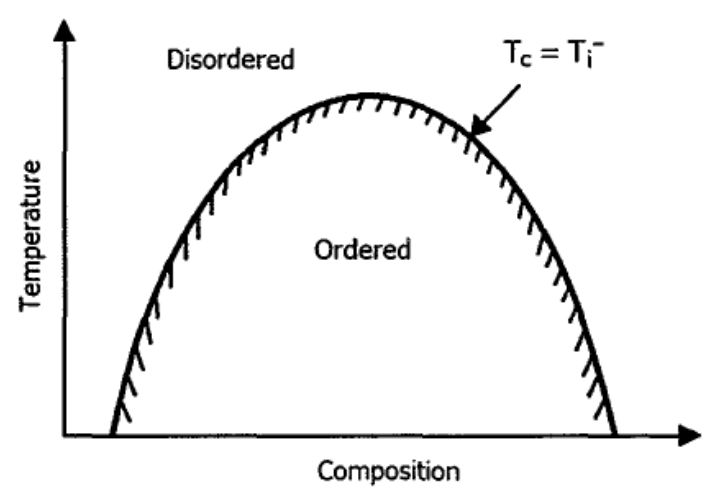

Figure 1.9: Schematic phase diagrams for (a) first order and (b) second/higher order disorderorder transformations. ${ }^{10}$

The change in free energy upon ordering has been described by Landau as a function of the order parameter using a power series expansion:

$\mathrm{G}(\eta)-\mathrm{G}(0)=\mathrm{A} \eta^{2}+\beta \eta^{3}+\tau \eta^{4}+\ldots$

where $G(\eta)$ is the free energy of the ordered phase associated with an order parameter $\eta$, at a given temperature $T, G(0)$ is the reference free energy of the disordered state, $A=\alpha\left(T-T_{0}\right)$, while $\alpha, \beta$ and $\tau$ are essentially constants.

The variation of free energy with order parameter for a first order phase transformation is shown in Figure 1.10 (a). In this case, $G(\eta)-G(0)=A \eta^{2}+\beta \eta^{3}+\tau \eta^{4}$ and $T_{o}=T_{i}^{-}$. At $T>T_{c}$, the minimum in the free energy versus order parameter curve occurs at $\eta=0$ and the disordered state is stable. As the alloy is cooled, a secondary minimum is seen to appear at $\eta>0$ and the phase corresponding to the lower energy minimum of the two will be stable. The two minima correspond to equal values of $G(\eta)-G(0)$ at $T=T_{c}$. The second minimum at $\eta>0$ will correspond to lower energy at $\mathrm{T}_{\mathrm{i}}^{-}<\mathrm{T}<\mathrm{T}_{\mathrm{c}}$, and the parent phase is metastable in that temperature range. The transformation proceeds by continuous ordering at $\mathrm{T}<\mathrm{T}_{\mathrm{i}}^{-19}$

The variation of free energy with order parameter for a second order phase transformation is schematically illustrated in Figure $1.10(\mathrm{~b})$. In this case, $G(\eta)-G(0)=A \eta^{2}+\tau \eta^{4} \cdot T_{o}=T_{c}=T_{i}^{-}$ 
At $\mathrm{T}>\mathrm{T}_{\mathrm{c}}$, the minimum in the free energy versus order parameter curve occurs at $\eta=0$ and the disordered state is stable. For $\mathrm{T}<\mathrm{T}_{\mathrm{c}}$, additional minima occur symmetrically at $|\eta|>0$. Thus, unlike in first order transformations, the disordered parent phase is unstable below $\mathrm{T}_{\mathrm{c}}$ and the ordered phase, which forms continuously, is the stable phase at $\mathrm{T}<\mathrm{T}_{\mathrm{c}}$.

a)
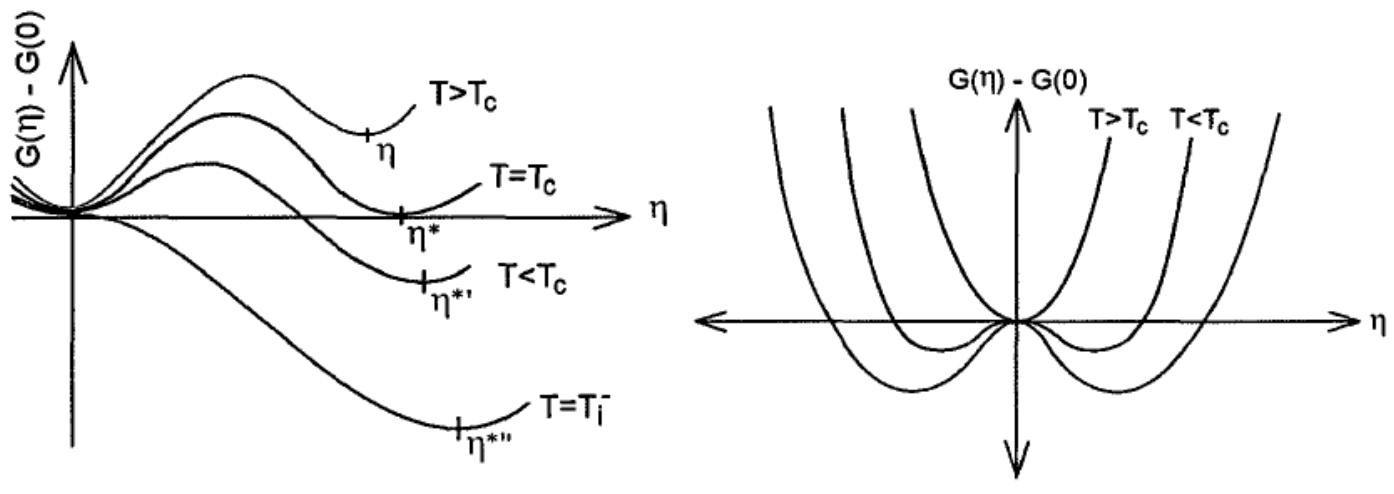

b)

Figure 1.10: Free energy versus order parameter curves according to Landau's expansion for (a) first order and b) second/higher order disorder-order transformations. ${ }^{10}$

\subsubsection{Eutectoid reaction and the nanochessboard structure in the Co-Pt system}

Apart from the phase transformations which yield either the $\mathrm{L} 1_{0}$ or the $\mathrm{L} 1_{2}$ phase based on the stoichiometry of the Co-Pt alloy, there exists a very interesting, largely unexplored region, highlighted in Figure 1.3, where the two ordered phases coexist. Compositions near the eutectoid composition $\left(\sim 60\right.$ atom \% Pt) undergo the eutectoid transformation $\mathrm{A} 1 \rightarrow \mathrm{L}_{0}+\mathrm{L}_{2}$ upon cooling through the eutectoid temperature, $730 \pm 5{ }^{\circ} \mathrm{C}$, to produce a mixture of the $\mathrm{L} 1_{0}$ and $\mathrm{L}_{2}$ ordered phases. According to this diagram, the eutectoid reaction isotherm spans a very narrow composition range, 59.5 to 61.5 atom $\% \mathrm{Pt}( \pm 0.5$ atom $\% \mathrm{Pt})$ and the two phase region extends from 59 to 63 atom \% Pt at low temperature. Leroux, et al., ${ }^{1}$ have demonstrated that appropriate thermal processing of near-eutectoid Co-Pt alloys results in the development of the two-phase mixture, possessing a characteristic nanochessboard microstructure, shown in the 110 Dark Field TEM image in Figure 1.11 (a).

Samples were first annealed at an elevated temperature, $930{ }^{\circ} \mathrm{C}$, and then water quenched to retain the disordered FCC phase. This was followed by continuous cooling from $740{ }^{\circ} \mathrm{C} @ 20$ 
${ }^{\circ} \mathrm{C} /$ day upto the temperature of interest followed by annealing at this temperature, ranging from 'a few days' at $700{ }^{\circ} \mathrm{C}$ to three weeks at $530{ }^{\circ} \mathrm{C}$, although the exact thermal treatment leading to the development of the chessboard structure in Figure 1.11 (a) was not reported ${ }^{1}$. The idealized chessboard structure is schematically illustrated in Figure 1.11 (b).

The nanochessboard structure contains alternating single crystal $\mathrm{L}_{2}$ and $\mathrm{L}_{0}$ rods which share a common parent lattice with coherent interfaces between the two phases. These parallel coherent rods are packed together on a 20-30 $\mathrm{nm}$ lengthscale and are aligned along one of the three cube axes of the parent phase, i.e., three spatial orientations of the chessboard are possible within a given grain of the parent A1 phase. Viewed end on, Dark Field TEM imaging reveals the dark and bright diamond-shaped tiling ${ }^{2}$, i.e., the 'chessboard' pattern, seen in Figures 1.11 (a). Here, the bright contrast comes from regions containing the $\mathrm{L} 1_{2}$ phase. The $\mathrm{L} 1_{0}$ phase is out of contrast; two orientation variants of the $\mathrm{L} 1_{0}$ phase, with mutually perpendicular $\mathrm{c}$ axes, are present in this structure, appearing as dark tiles in these images. ${ }^{1}$ The $\mathrm{c}$ axis of the $\mathrm{L} 1_{0}$ nanorods is perpendicular to the rod axis. ${ }^{2}$ Viewing these chessboards side-on would show the nanorods instead of the tiling. This can be easily understood from Figure 1.1.

A more accurate depiction of the tile geometry is shown in Figure 1.11 (c). The orientation relationship between the $\mathrm{L} 1_{0}$ and $\mathrm{L} 1_{2}$ tiles is (110) $\mathrm{L}_{0} \|(110) \mathrm{L}_{2}$ and [100] $\mathrm{L}_{0} \|$ [100] $\mathrm{L1}_{2}$. The $\mathrm{L} 1_{0} / \mathrm{L1}_{2}$ interfaces are close to the $\{110\}$ planes, with a deviation of $\pm 11^{\circ}$. The 'tiles' form zigzag bands along $\langle 110\rangle$ with alternate bands containing tiles of $\mathrm{L1}_{2}+\mathrm{X}$ variant of $\mathrm{L1}_{0}$ and $\mathrm{L}_{2}+\mathrm{Y}$ variant of $\mathrm{L} 1_{0}$, respectively. ${ }^{1}$ It may be noted that only $\mathrm{L} 1_{0}$ and $\mathrm{L}_{2}$ phases share interfaces; $\mathrm{L1}_{0}$ orientation variants do not share common interfaces.

Le Bouar et al. $^{2}$ have reported this nanochessboard structure in a $\mathrm{Co}_{39.5} \mathrm{Pt}_{60.5}$ alloy, shown in Figure 1.12 , which was slow cooled from 750 to $650^{\circ} \mathrm{C}$, then held $650{ }^{\circ} \mathrm{C}$ for 15 days, followed by water quenching. 

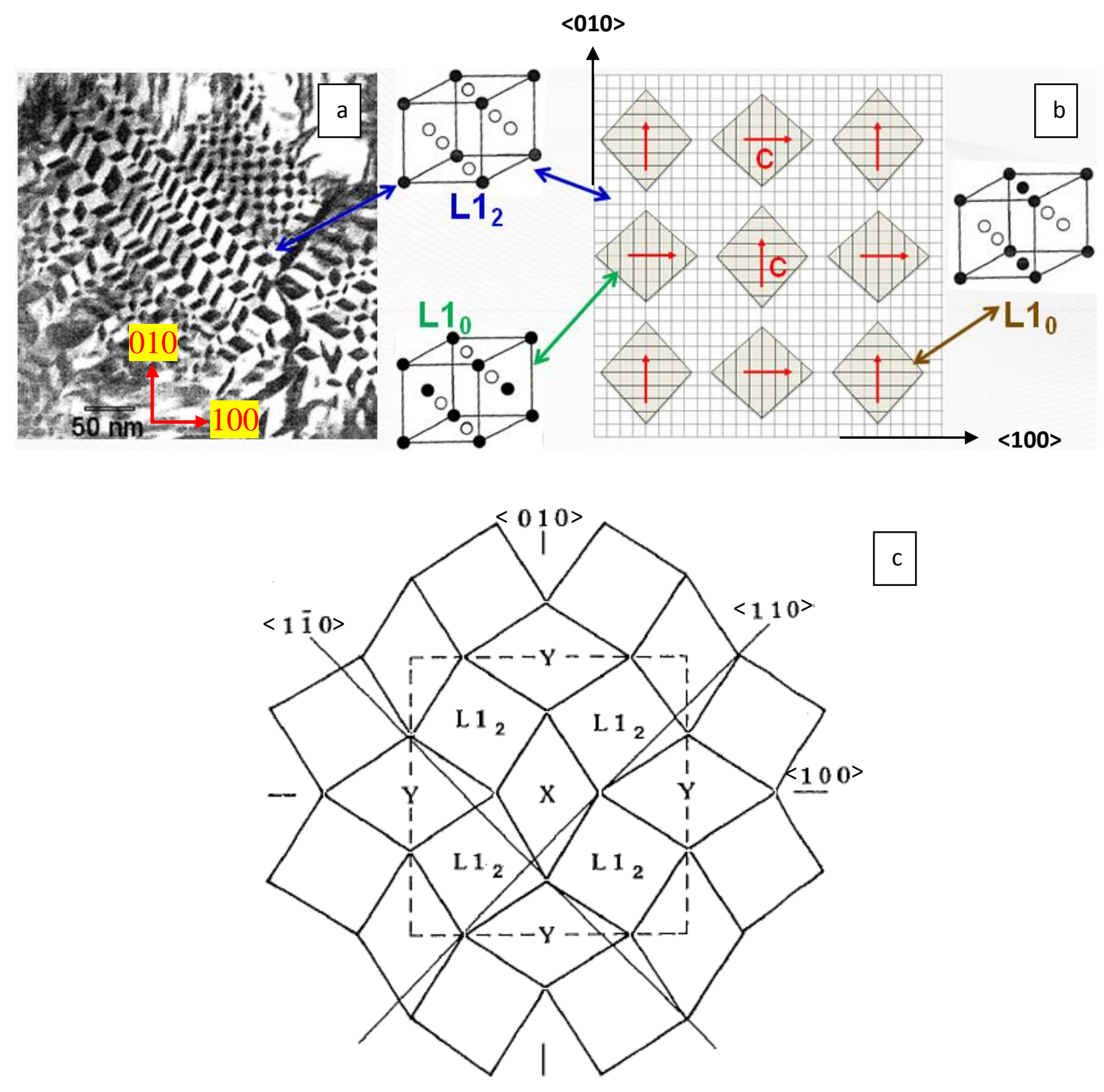

Figure 1.11: (a) 110 dark field image of the nanochessboard structure in a near-eutectoid Co-Pt alloy viewed along the [001] zone axis, obtained by Leroux et al. ${ }^{1}$. The bright tiles correspond to the $\mathrm{L}_{2}$ phase while the dark tiles correspond to the $\mathrm{L}_{0}$ phase. (b) Schematic, idealized representation of the chessboard structure seen in (a), highlighting the crystallography of the constituent structural domains. ${ }^{2}$ Red arrows represent the c-axis directions of $\mathrm{L} 1_{0}$. (c) More accurate depiction of the tile geometry. 


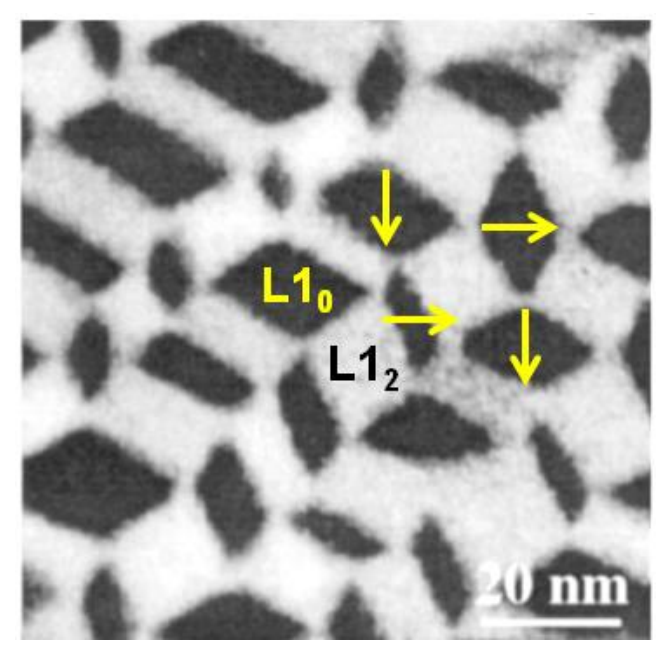

Figure 1.12: Nanochessboard structure with tiling of ferromagnetic soft $\mathrm{L}_{2}$ and hard $\mathrm{L} 1_{0}$ phases, on a $20 \mathrm{~nm}$ lengthscale, obtained by Le Bouar et al. ${ }^{2}$ Yellow arrows represent the c-axis directions of $\mathrm{L} 1_{0}$.

\subsubsection{Characterizing the presence of ordered phases}

The disorder - order transformation can be detected from x-ray diffraction (XRD) patterns or from selected area diffraction patterns (SADPs) obtained using TEM. The structure factor (F) accounts for the arrangements of atoms within a unit cell to describe the resultant wave scattered by the atoms in the unit cell. ${ }^{20}$ Structure factor calculations determine allowed reflections and the reflection intensity is directly proportional to $\mathrm{F}^{2}$. In XRD patterns, these reflections will be manifested as diffraction peaks and in case of SADPs, they will be seen as diffraction spots.

The structure factor for the disordered FCC phase in a Co-Pt alloy, for a plane (hkl) is given as:

$$
\mathrm{F}_{\mathrm{FCC}}=\mathrm{f}_{\mathrm{av}}\{1+\exp [-\pi \mathrm{i}(\mathrm{h}+\mathrm{k})]+\exp [-\pi \mathrm{i}(\mathrm{k}+\mathrm{l})]+\exp [-\pi \mathrm{i}(\mathrm{l}+\mathrm{h})]\} \quad \ldots(\mathrm{Eq} .1 .3)
$$

$\mathrm{f}_{\mathrm{av}}$ being the atomic scattering factor of the average atom. Reflections corresponding to unmixed hkl, i.e., where h,k,l are either all odd or all even, such as $\{111\},\{200\}$ etc., have a structure factor of $4 \mathrm{f}_{\mathrm{av}}$ and are thus present in the diffraction pattern; these are called fundamental reflections. Reflections corresponding to mixed hkl, such as $\{100\},\{110\}$ etc., have a structure factor of 0 and are thus absent in the diffraction pattern of the disordered phase; these are called 
superlattice reflections since they can occur in the ordered phases due to a change in the translational symmetry.

Ordering produces weak superlattice reflections and hence, the diffraction patterns of ordered phases contain these in addition to the fundamental reflections. Superlattice reflections are weaker in intensity compared to the fundamental reflections since their structure factors involve a difference between the atomic scattering factors (f) of constituent atoms as opposed to their sum in case of fundamental reflections. ${ }^{20}$

The structure factors for the orientation variants of the conventional stoichiometric $\mathrm{L}_{0}(\mathrm{CoPt})$ unit cell shown in Figure 1.7 are:

$\mathrm{F}_{\mathrm{L} 10-\mathrm{Z}}=\mathrm{f}_{\mathrm{Co}}\{1+\exp [-\pi \mathrm{i}(\mathrm{h}+\mathrm{k})]\}+\mathrm{f}_{\mathrm{Pt}}\{\exp [-\pi \mathrm{i}(\mathrm{k}+\mathrm{l})]+\exp [-\pi \mathrm{i}(1+\mathrm{h})]\}$

$\mathrm{F}_{\mathrm{L} 10-\mathrm{X}}=\mathrm{f}_{\mathrm{Co}}\{1+\exp [-\pi \mathrm{i}(\mathrm{k}+\mathrm{l})]\}+\mathrm{f}_{\mathrm{Pt}}\{\exp [-\pi \mathrm{i}(\mathrm{h}+\mathrm{k})]+\exp [-\pi \mathrm{i}(1+\mathrm{h})]\}$

$\mathrm{F}_{\mathrm{L} 10-\mathrm{Y}}=\mathrm{f}_{\mathrm{Co}}\{1+\exp [-\pi \mathrm{i}(1+\mathrm{h})]\}+\mathrm{f}_{\mathrm{Co}}\{\exp [-\pi \mathrm{i}(\mathrm{h}+\mathrm{k})]+\exp [-\pi \mathrm{i}(\mathrm{k}+\mathrm{l})]\}$

Let us consider the $\{100\}$ reflection. Eq. 1.4 indicates that while the (001) reflection is permitted for the Z-variant of the $\mathrm{L}_{0}$ structure, (100) and (010) are not. Similarly, from Eq. 1.5, the (100) reflection is present in the $\mathrm{X}$ variant, but not (010) or (001); and from Eq. 1.6, the (010) reflection is allowed in the Y variant, but not (100) or (001). However, all these reflections from the orientation variants correspond to the ordering half plane, with identical values of the interplanar spacing ' $d$ ', where $d=c / 2$. The three orientation variants will thus contribute to a single $\{100\}$ peak in an XRD pattern. Similarly, all peaks, superlattice as well as fundamental, contain contributions from all three orientation variants.

The d-spacing for a plane $\{h \mathrm{kl}\}$ in an FCC structure with a lattice parameter 'a' is given as:

$\mathrm{d}_{\mathrm{FCC}}=\mathrm{a} / \sqrt{ }\left(\mathrm{h}^{2}+\mathrm{k}^{2}+\mathrm{l}^{2}\right)$

The d-spacing for a plane $\{\mathrm{hkl}\}$ in an $\mathrm{L} 1_{0}$ structure with lattice parameters ' $\mathrm{a}$ ', 'c' is given as:

$\mathrm{d}_{\mathrm{L} 10}=1 /\left\{\mathrm{a} / \sqrt{ }\left[\left(\mathrm{h}^{2} / \mathrm{a}^{2}\right)+\left(\mathrm{k}^{2} / \mathrm{a}^{2}\right)+\left(\mathrm{1}^{2} / \mathrm{c}^{2}\right)\right]\right\}$

Due to tetragonality, each orientation variant of the $\mathrm{L} 1_{0}$ phase, contributes to split $\{200\} /(002)$ peaks in the XRD diffraction pattern, since they correspond to different d spacings, respectively 
a and c, as can be seen from equations 1.7 and 1.8. In general, except for those with identical values of h,k,l like $\{111\},\{222\}$ etc., fundamental reflections, for e.g., $\{202\} /(220),\{311\} /(113)$, exhibit peak splitting. Superlattice reflections from the $\mathrm{L} 1_{0}$ phase do not undergo this peak splitting, on the basis of the structure factor calculations.

In case of the stoichiometric $\mathrm{L1}_{2}\left(\mathrm{CoPt}_{3}\right)$ unit cell shown in Figure 1.3,

$\mathrm{F}_{\mathrm{L} 12}=\mathrm{f}_{\mathrm{Co}}+\mathrm{f}_{\mathrm{Pt}}\{\exp [-\pi \mathrm{i}(\mathrm{h}+\mathrm{k})]+\exp [-\pi \mathrm{i}(\mathrm{k}+\mathrm{l})]+\exp [-\pi \mathrm{i}(\mathrm{l}+\mathrm{h})]\}$

$F_{\mathrm{L} 12}$ equals $\left(\mathrm{f}_{\mathrm{Co}}+3 \mathrm{f}_{\mathrm{Pt}}\right)$ for fundamental reflections, i.e., for unmixed hkl. For superlattice reflections, i.e., for mixed hkl, $\mathrm{F}_{\mathrm{L} 12}$ equals $\left(\mathrm{f}_{\mathrm{Co}}-\mathrm{f}_{\mathrm{Pt}}\right) .{ }^{20}$ Peak splitting is not observed as the $\mathrm{L}_{2}$ unit cell is cubic, unlike tetragonal $\mathrm{L}_{0}{ }^{20}$

Thus, the presence of superlattice reflections is evidence for the presence of an ordered phase. Furthermore, in a single phase ordered Co-Pt alloy, the splitting of fundamental XRD peaks clearly indicates the presence of the $\mathrm{L} 1_{0}$ phase. Lack of peak splitting in spite of the presence of superlattice reflections indicates either an $\mathrm{L}_{2}$ phase or an $\mathrm{L} 1_{0}$ phase with very low tetragonality. Apparent peak splitting in a superlattice reflection is seen when both ordered phases are simultaneously present, such as in the chessboard structure.

A schematic [001] SADP from an area containing all three orientation variants of the $\mathrm{L}_{0}$ phase is schematically illustrated in Figure 1.13 (a). The yellow diffraction spots are the fundamental reflections whereas the red diffraction spots correspond to the superlattice reflections and would be absent in the disordered phase. The 100 diffraction spot corresponds to the $\mathrm{X}$ variant, the 010 spot to the $\mathrm{Y}$ variant whereas the 110 spot corresponds to the $\mathrm{Z}$ variant. Dark field imaging using these superlattice reflections allows determination of regions containing the ordered phase. The regions corresponding to one particular variant, say the $\mathrm{X}$ variant, will light up in a dark field image formed using the corresponding diffraction spot, here $\mathbf{g}=100$, while the regions containing the other two variants of the $\mathrm{L}_{0}$ phase or the disordered phase will remain out of contrast. If only one orientation variant is present, say the $\mathrm{X}$-variant, only the superlattice reflection corresponding to it, 100, will be present in the SADP while the 010 and 110 reflections would be absent. 
The schematic illustration in Figure 1.13(a) would also correspond to the SADP from a region containing the $\mathrm{L}_{2}$ phase. Being cubic, the $\mathrm{L}_{2}$ phase will not have orientation variants and thus, the 100,010 and 110 reflections must simultaneously be present if the $\mathrm{L} 1_{2}$ phase is present; if not, the ordered phase corresponding to the SADP cannot be $\mathrm{L}_{2}$. Dark field TEM using any one of these reflections will lead to the entire $\mathrm{L}_{2}$ region lighting up.

As described above, the nanochessboard structure contains the $\mathrm{L}_{2}$ phase and two orientation variants of the $\mathrm{L}_{0}$ phase. The $\mathrm{L}_{0}$ orientation variants in question would be $\mathrm{X}$ and $\mathrm{Y}$ for an endon chessboard viewed along the [001] direction. Let us consider the [001] SADP from a region containing this structure, shown in Figure 1.13 (b) and schematically illustrated and labeled in Figure 1.13 (c), where we see all three superlattice reflections discussed above. The $\mathrm{X}$ and $\mathrm{Y}$ variants of the $\mathrm{L} 1_{0}$ phase present in this structure would contribute to the 100 and 010 diffraction spots, respectively, but the absence of the third orientation variant in this structure implies that the $\mathrm{L}_{0}$ phase does not contribute to the 110 diffraction spot intensity. The $\mathrm{L}_{2}$ phase will contribute to the 100, 010 and 110 diffraction spot intensities. Thus, all phases present: any untransformed FCC, as well as the ordered $\mathrm{L1}_{0}$ and $\mathrm{L1}_{2}$ phases, would contribute to the fundamental reflections, indicated by yellow circles in Figures 1.13 (b), (c). The 100 and 010 spots, indicated by red circles, would arise from both ordered phases: $\mathrm{L} 1_{0}$ as well as $\mathrm{L} 1_{2}$ whereas the 110 spot, indicated in green, corresponds exclusively to the $\mathrm{L1}_{2}$ phase. Thus, the $\mathrm{L1}_{2}$ phase will exclusively exhibit bright contrast in a 110 DF image for an area viewed along the [001] zone axis. Dark field images formed with superlattice reflections reveal the tiling characteristic of end-on chessboards. 


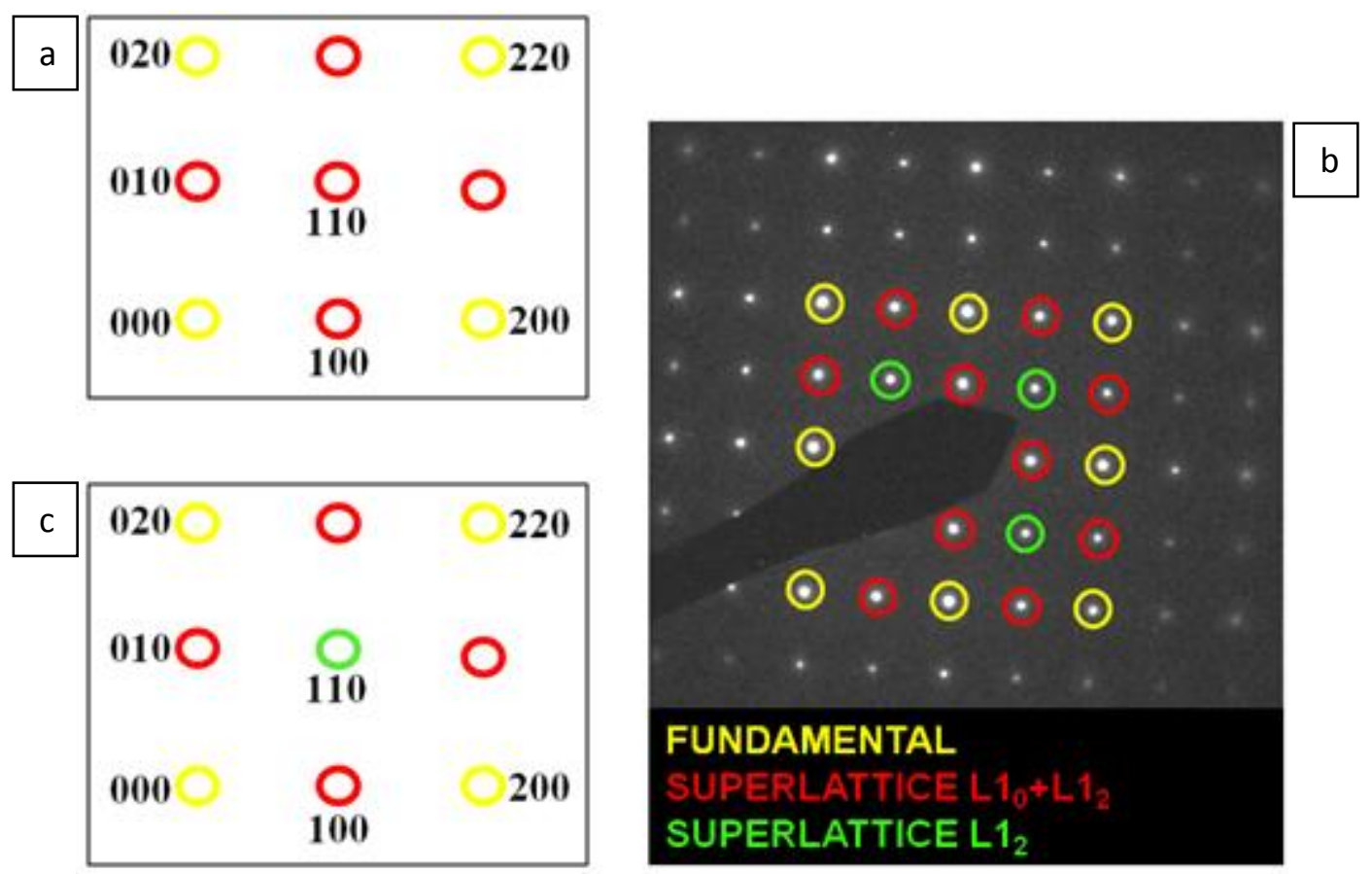

Figure 1.13: (a) Schematic [001] SADP corresponding to a region containing all three orientations of the $\mathrm{L}_{0}$ phase or containing the $\mathrm{L1}_{2}$ phase. (b) [001] SADP and (c) schematic [001] SADP corresponding to the chessboard microstructure.

\subsection{Evolution of Microstructure}

The ordering transformation is accompanied by the formation of several internal interfaces due to the existence of multiple variants of the ordered phase. ${ }^{21}$ Typical microstructures that develop in Co-Pt alloys undergoing the disorder - order transformation are described below.

\section{$\underline{1.3 .1 \mathrm{FCC} \rightarrow \mathrm{L}_{\underline{0}}}$}

Alloys that transform from the disordered FCC structure to the ordered $\mathrm{L}_{0}$ structure upon cooling below the critical temperature $\mathrm{T}_{\mathrm{c}}$ (Figure 1.2) are Co-rich with respect to the composition range of the eutectoid isotherm (Figure 1.3). The microstructural evolution in equiatomic ordering systems such as CoPt, FePd and Fe-37Pt alloys has been the most widely studied of such alloys. In such alloys, the transformation starts with the formation and alignment of the $\mathrm{L}_{0}$ 
nuclei on the $\{110\}$ planes, along $<110>$ directions in the disordered matrix. ${ }^{22}$ These tetragonal strain centers result in a nanoscale-modulated matrix strain responsible for "tweed contrast" observed in the early stages of transformation, as shown in Figure 1.14. Initially, particles of all three $\mathrm{L} 1_{0}$ orientation variants are formed. However, one variant is annealed out and two variants persist through further development and coarsening of the structure. This produces the alternating bands shown in Figure 1.15, with alternate bands containing particles of alternate orientation variants.

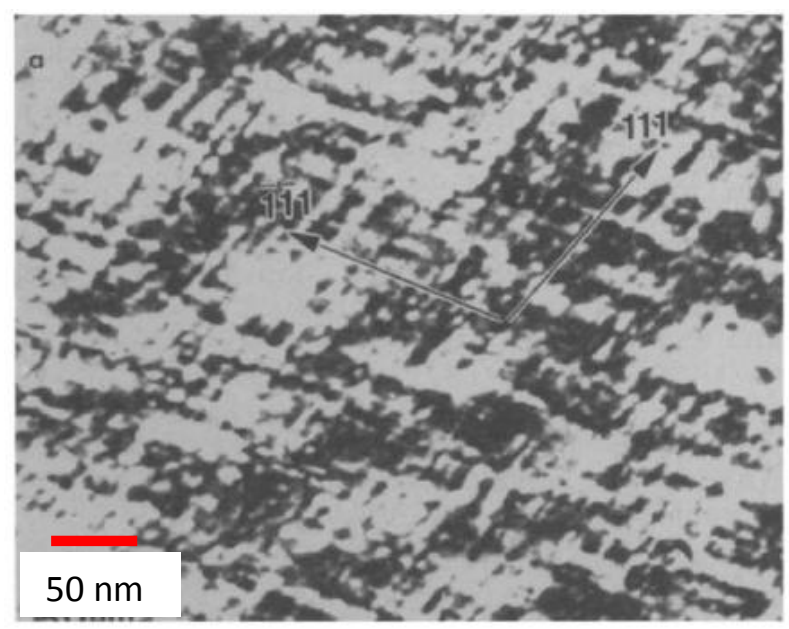

Figure 1.14: Tweed contrast in Fe-Pt alloy. ${ }^{23}$
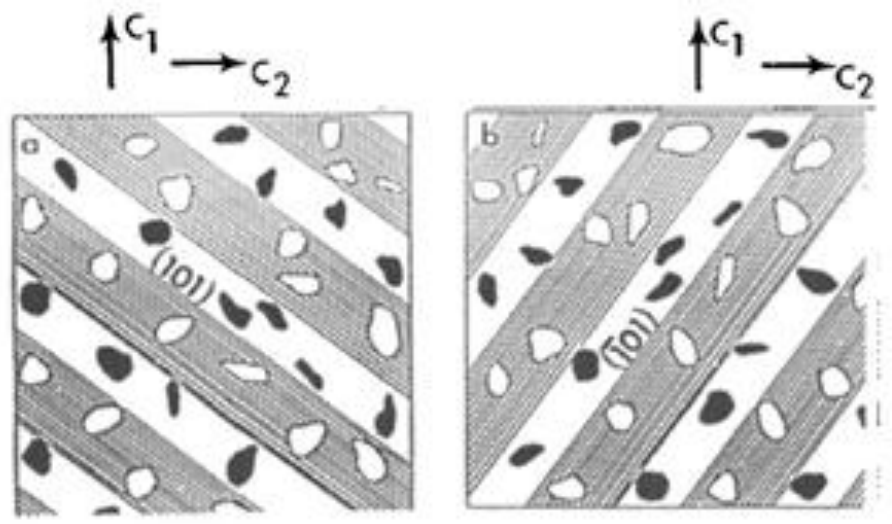

Figure1.15: Schematic diagram showing the alignment, distribution of the $\mathrm{L} 1_{0}$ precipitates on alternating $\{110\}$ bands. The dark and bright particles have mutually perpendicular c axes, $\mathrm{c}_{1}$ and $c_{2}$ and lie on alternating regularly spaced bands; $a$ and $b$ are two possible configurations of the $\{110\}$ bands on which these particles are distributed. Note that the black and white bands are only meant to indicate the regions over which particles will coalesce. ${ }^{23,24}$ 
With continued annealing, the particles impinge and coalesce in the $\{110\}$ bands forming fine parallel plate-shaped c-variants or 'microtwins' (since they have a formal twining relation). This impingement and coalescence of particles results in a high density of APBs. ${ }^{22,} 25$ These microtwins are conjugated along $\{110\}$ planes (which produce invariant plane strain), creating a modulation of the $\mathrm{c}$ axis across the twin related regions on a scale of 10-100 $\mathrm{nm}$. The $\{110\}$ microtwin boundaries are coherent interfaces by the mutual rotation of the c axes, such that they are misoriented by $88^{\circ}$ (as opposed to $90^{\circ}$ ) across the $\{110\}$ microtwin interface. ${ }^{22}$ This is schematically illustrated in Figure 1.16.

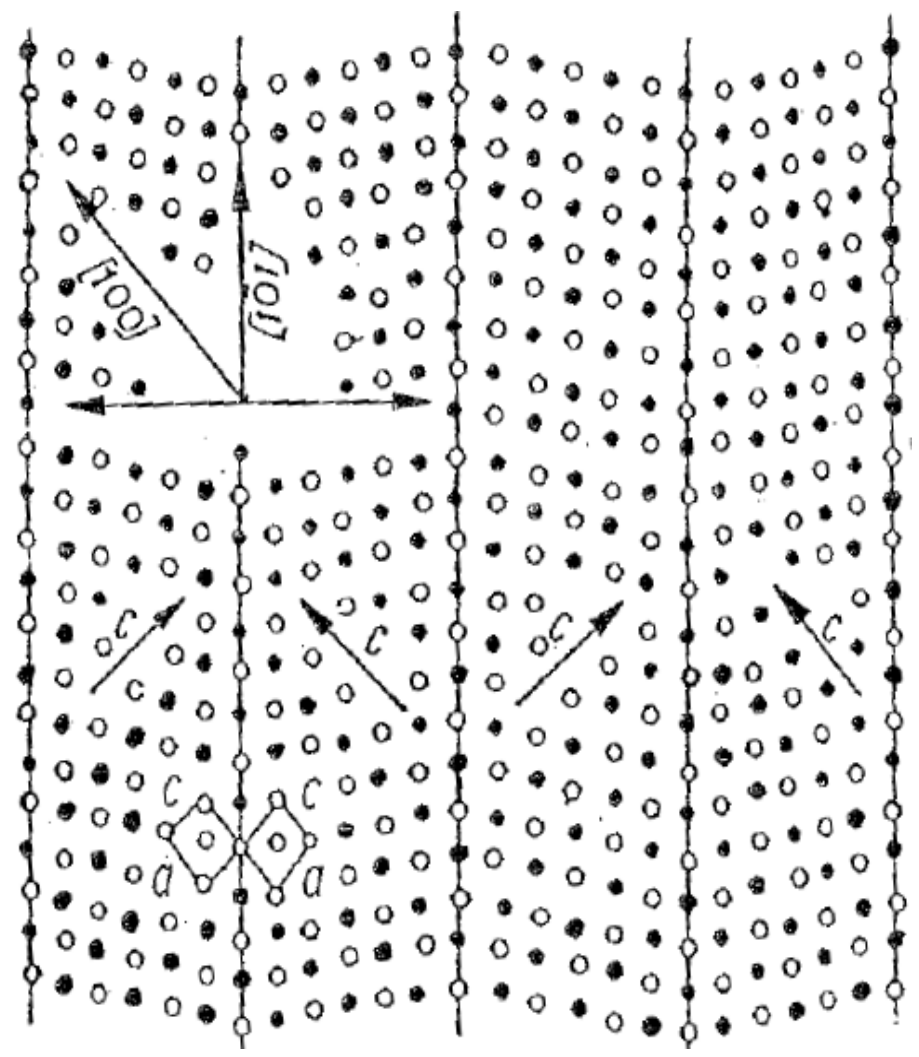

Figure 1.16: Schematic diagram depicting the atomic rearrangement in microtwins and strain free microtwin boundaries within a macrotwin. $\mathrm{C}$ axes are misoriented by $88^{\circ}$ across the microtwin boundary. ${ }^{26}$

Figure 1.17 shows a schematic representation of a "polytwinned" crystal containing fine packets of polytwinned plates that grew from many centers, occurring when the rate of growth of transformation centers is significantly larger than their rate of nucleation. A hierarchy of 
structural elements is observed here. A row of parallel microtwins of thickness $\mathrm{d} \approx 0.1 \mu \mathrm{m}$ comprises a polytwinned plate/macrotwin of thickness $\mathrm{L}_{1} \approx 1-5 \mu \mathrm{m}$. Extended packets (or colonies) of parallel polytwinned plates can have a thickness $\mathrm{L} \approx 50-100 \mu \mathrm{m} .{ }^{21} \mathrm{TEM}$ images of the polytwinned structure in $\mathrm{L} 1_{0}$ alloys are shown in Figure 1.18. The polytwinned structure forms in order to accommodate the transformation strain arising from ordering. ${ }^{22}$

In case of rapid nucleation of transformation centers, a finely dispersed twinned microstructure is obtained. In this case, the hierarchy of structures consists of the microtwins or c-domains, blocks of c-domains and groups of blocks of c-domains; where the dimensions of blocks of microtwins are comparable to the dimensions of the microtwins, i.e., fractions of a micron. ${ }^{21}$

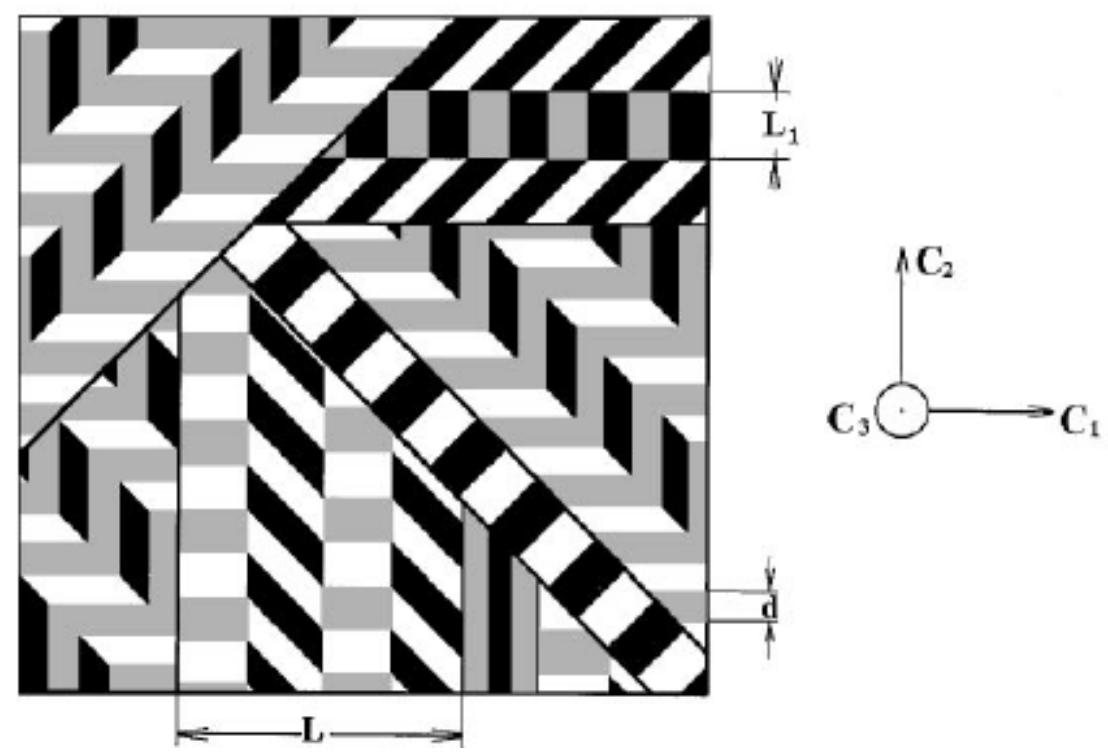

Figure 1.17: Schematic representation of the polytwinned crystal, where the colors white, gray and black are used to represent the three orientation variants of the ordered $\mathrm{L} 1_{0}$ phase. ${ }^{27}$ 

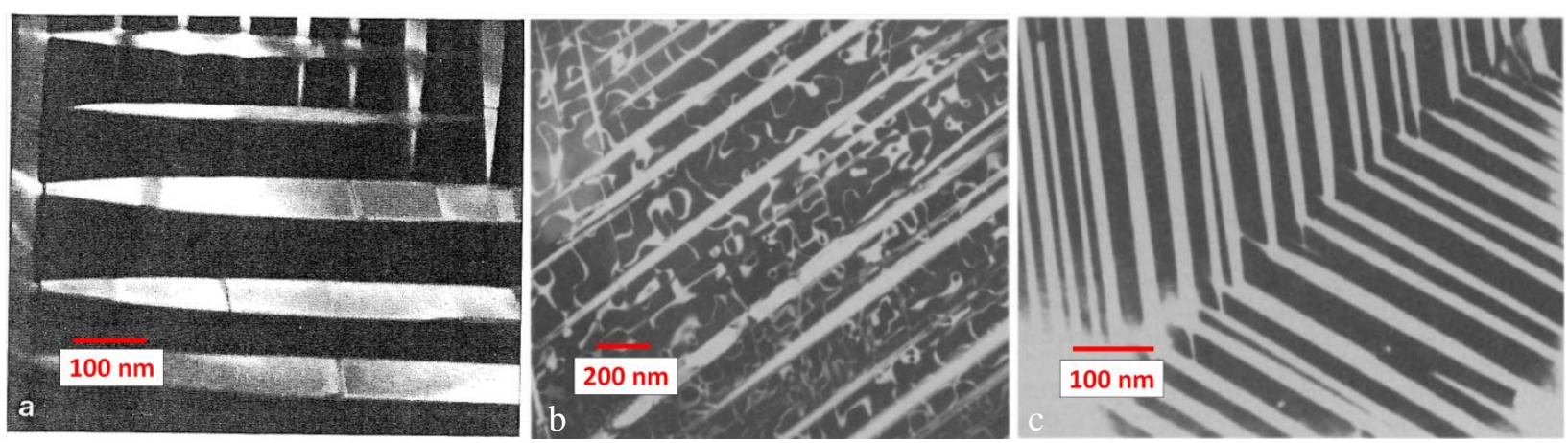

Figure 1.18: Polytwinned structure in $\mathrm{L}_{0}$ alloys: (a) $100 \mathrm{DF}$ image showing microtwins in $\mathrm{CoPt}^{1}$, (b) BF image showing microtwins in FePd; profusion of APBs may be noted and (c) two macrotwins/polytwinned plates containing microtwins in FePd. ${ }^{23}$

\section{$\underline{\text { 1.3.2 } \mathrm{FCC} \rightarrow \mathrm{L1}_{2}}$}

In case of the disorder - order transformation from $\mathrm{FCC}$ to $\mathrm{L}_{2}$, large domains, containing numerous APBs are formed. ${ }^{1}$ This is illustrated in Figure 1.19.

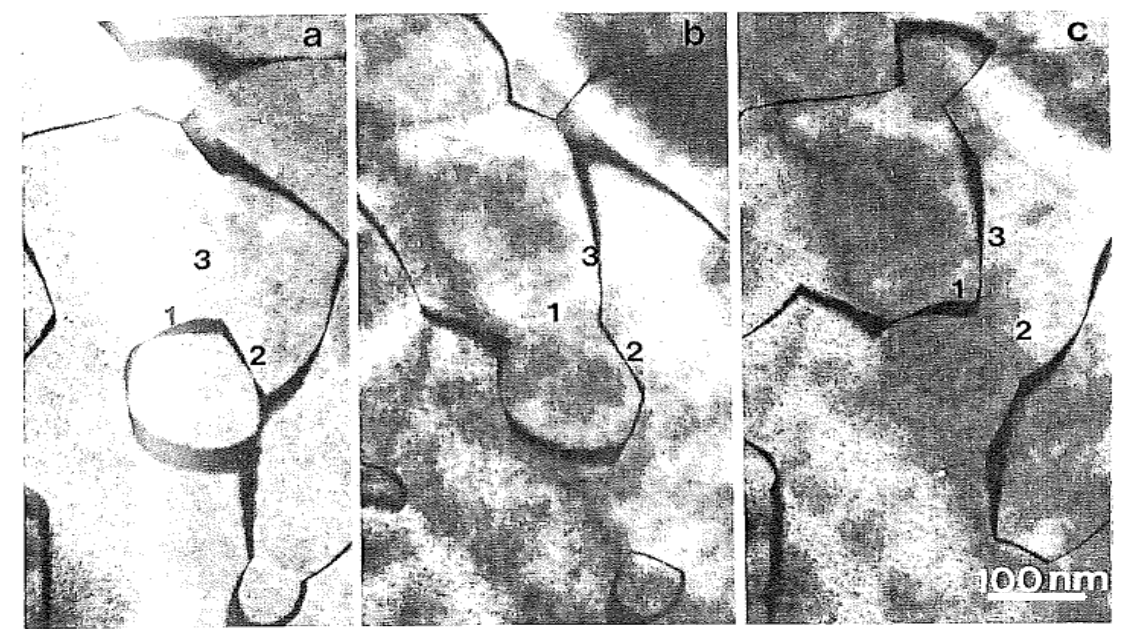

Figure 1.19: (a) $110 \mathrm{DF}$, (b) $010 \mathrm{DF}$ and (c) $100 \mathrm{DF}$ images of $\mathrm{CoPt}_{3}$ oriented along [001], showing characteristic $\mathrm{L}_{2}$ microstructure containing APBs. ${ }^{1}$ 


\section{$\underline{1.3 .3 \mathrm{FCC} \rightarrow \mathrm{L}_{1}} \underline{\underline{0}}+\underline{\mathrm{L}_{2}} \underline{\underline{2}}$}

The eutectoid decomposition of Co-Pt: FCC $\rightarrow \mathrm{L}_{0}+\mathrm{L1}_{2}$, can lead to the formation of the chessboard microstructure. This microstructure results from strain-driven assembling of the $\mathrm{L} 1_{0}$ and $\mathrm{L}_{2}$ phases. Microstructural evolution is governed by the minimization of the interfacial energy and the coherency strain energy arising from the disorder-order transformation. ${ }^{2}$

According to theoretical and experimental studies by Le Bouar et al. ${ }^{2}$, the early stages in this phase transformation involve formation and alignment of $\mathrm{L}_{0}$ nuclei along the $\langle 110\rangle$ directions, giving rise to the tweed contrast seen in Figures 1.20 (a), (d). Small, irregularly shaped structural domains of the $\mathrm{L}_{0}$ phase aligned along <110> directions are seen in the next stage, as shown in Figures 1.20 (b), (e). Le Bouar et al. predict that APBs would be annealed out in early stages, being energetically unfavorable compared to matrix-precipitate interfaces. Upon further annealing, the structural domains eventually assume a diamond-shape with facets normal to elastically soft directions, as shown in Figures 1.20 (c), (f). These 'tiles' are arranged in alternating bands/rows (where in each row, the $\mathrm{L} 1_{0}$ tiles have the same c-axis orientation, and where adjacent rows alternate the c-axis orientation), indicated by red arrows in Figure 1.20 (c); these bands/rows of tiles vary in size. Ideally, with continued annealing, the tiles become nearly homogeneous in size and shape. This is a metastable state, that can be destroyed by coarsening, which proceeds by the disappearance of rows of tiles. ${ }^{2}$

This microstructural development is entirely in keeping with the pseudospinodal decomposition mechanism. Hence, we may conclude that the formation of the nanochessboard structure via eutectoid decomposition in Co-Pt alloys proceeds by pseuodospinodal decomposition. 2,28 Interestingly, the theory of Le Bouar et al. $^{2}$ does not explain how the disordered cubic matrix transforms to $\mathrm{L} 1_{2}$ but focuses on annealing in the $\mathrm{A} 1+\mathrm{L} 1_{0}$ coexistence region. Since the $\mathrm{L} 1_{2}$ phase is never considered, the theory does not truly describe the eutectoid decomposition process; nevertheless it does discuss the transformation for the case of a pseudospinodal reaction. 


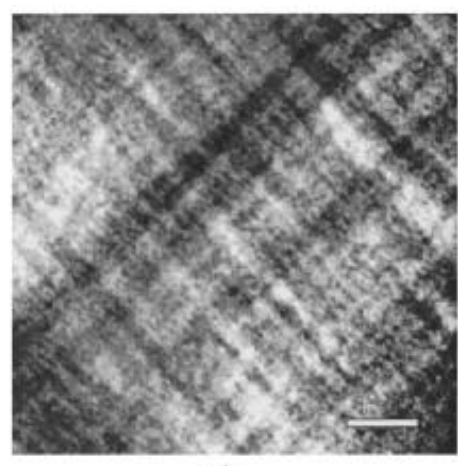

a)

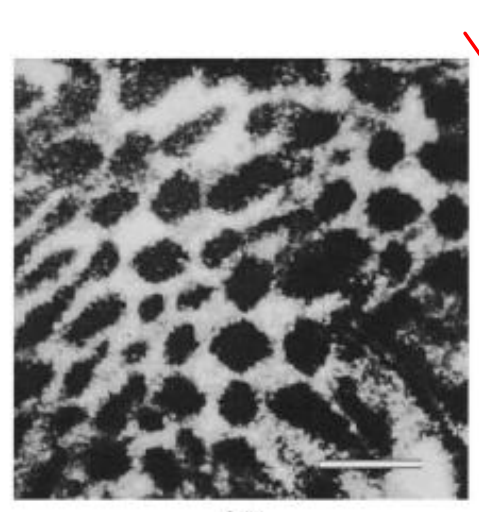

b)

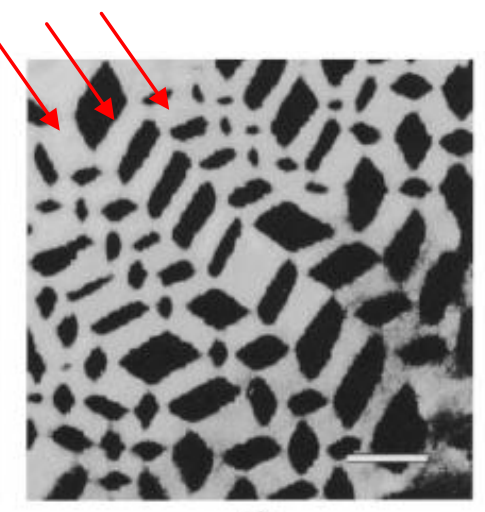

c)

Time

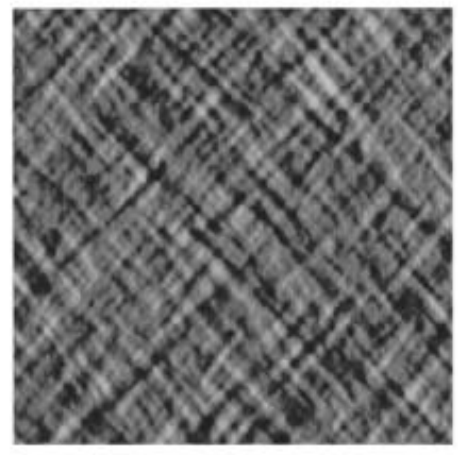

d)

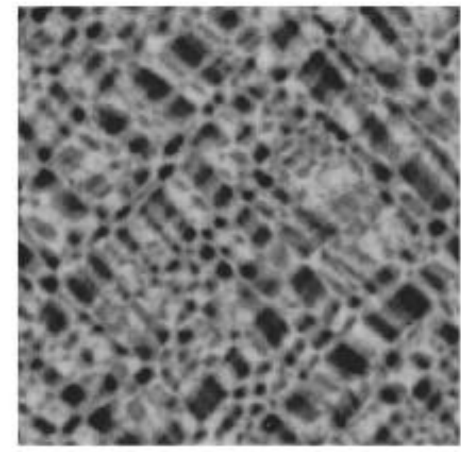

e)

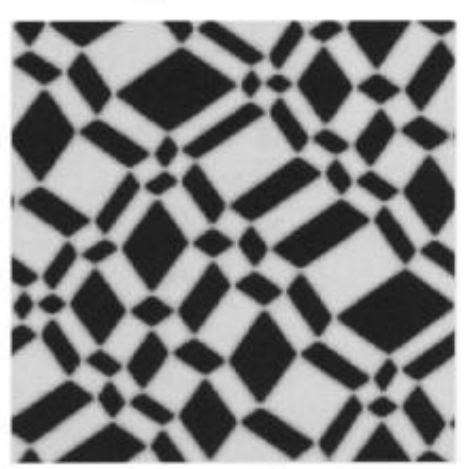

f)

Figure 1.20: Stages of microstructural development in the formation of the nanochessboard structure based on (a),(d),(f) experimental results and (b),(e),(f) simulation results. ${ }^{2}$ (a),(d) reveal tweed contrast, (b),(e) reveal irregular $\mathrm{L1}_{0}$ domains and (c),(f) reveal the chessboard structure.

The pseudospinodal mechanism has been discussed in the context of nucleation of the ordered $\mathrm{L1}_{0}$ phase in the supersaturated parent FCC phase, wherein the initial nuclei emerge with significantly non-equilibrium compositions due to small composition fluctuations; thereafter the compositions of the matrix and nuclei continuously change towards the equilibrium compositions upon further annealing. ${ }^{29}$ Thus, this reaction brings around a simultaneous change in composition and lattice arrangement via fluctuations of composition and the long range order parameters. Like the true spinodal mechanism, the pseudospinodal mode of transformation involves a continuous change in the compositions of the decomposed phases towards their equilibrium values. The difference between the two is that the decomposed phases arising from the pseudospinodal reaction have symmetries different from those of the parent phase whereas 
the products of spinodal decomposition have the same symmetries as the parent phase. Unlike pseudospinodal or spinodal decomposition reactions, conventional decomposition occurring via nucleation and growth leads to the formation of precipitates with composition nearly equal to equilibrium value, right from the outset. ${ }^{3}$

$\mathrm{Ni}$ and Khachaturyan ${ }^{28}$ specifically analyzed the cubic $(\alpha) \rightarrow$ cubic $(\alpha)+$ tetragonal $(\beta)$ transformation, where the cubic parent phase produces a coherent mixture of the product cubic and tetragonal phases (note that the product cubic phase in this case is the same structure as the parent phase, but will have a different composition in equilibrium). Consider the corresponding free energy curves of the cubic and tetragonal phases in an alloy undergoing the cubic $\rightarrow$ tetragonal disorder to order transformation at a temperature T, shown in Figure 1.21.

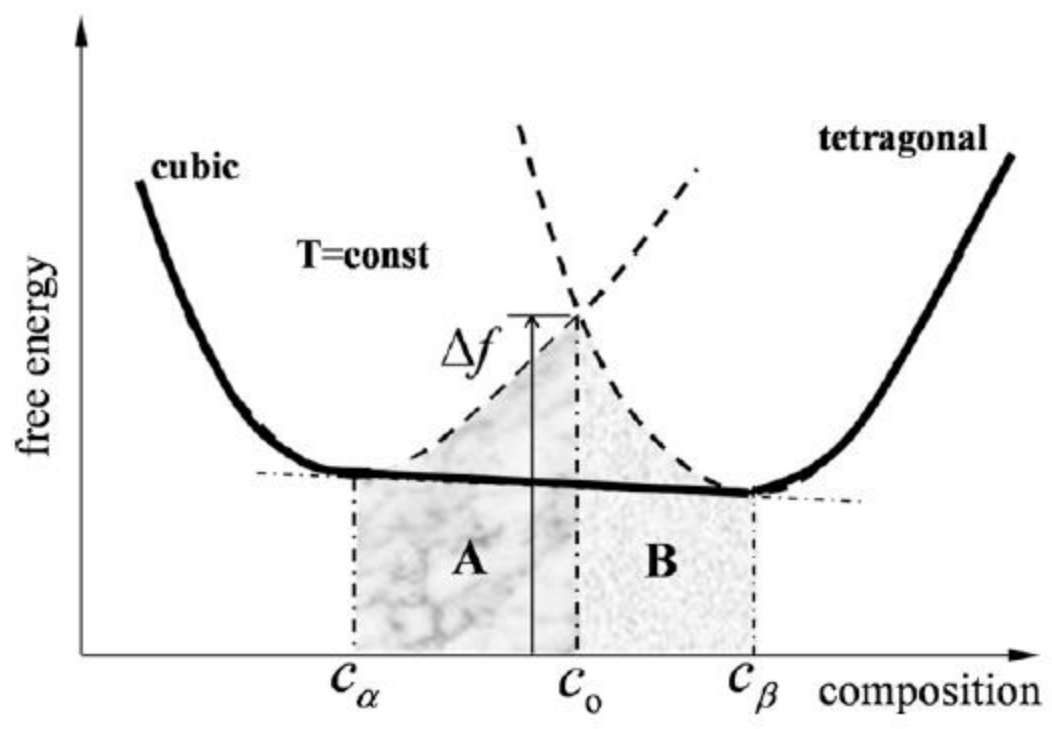

Figure 1.21: Schematic plot showing the variation of free energy with composition for coherent cubic, tetragonal phases, at a temperature $\mathrm{T}^{28}$

The point of intersection of the two curves at $\mathrm{T}$, corresponding to equal free energies of both phases is referred to as the crossover, $\mathrm{c}_{\mathrm{o}}$. For a given system composition $\mathrm{c}^{\prime}$, the free energy of the cubic phase is lower than the free energy of the tetragonal phase for $c^{\prime}<c_{o}$ while the free energy of the tetragonal phase is lower for $c^{\prime}>c_{o}$ in the two phase field considered here. The 
system composition with respect to $\mathrm{c}_{\mathrm{o}}$ governs the phase transformation sequence and the resultant microstructure, as described below for compositions in the vicinity of the crossover.

For the free energy curves shown in Figure 1.21, a diffusionless cubic-tetragonal transformation at constant composition cannot occur for $\mathrm{c}^{\prime}<\mathrm{c}_{\mathrm{o}}$, on account of the lower energy of the cubic phase with respect to the tetragonal phase. Instead, it is initiated by nucleation of the tetragonal phase, which requires simultaneous lattice rearrangement and change in composition. Ni and Khachaturyan's ${ }^{28}$ simulations confirm the formation of tweed, wherein the nuclei of the ordered phase differ only slightly in composition from the matrix, leading up to the chessboard microstructure, as shown in Figure 1.22 (A). This confirms that the mechanism of phase transformation in this regime is pseudospinodal decomposition. The tweed structure is found to have the same geometry as the chessboard.

On the other hand, for $\mathrm{c}^{\prime}>\mathrm{c}_{\mathrm{o}}$, where the tetragonal phase is associated with lower free energy, the transformation starts as a congruent lattice rearrangement via either displacive transformation or ordering, instead of by the much slower long range diffusion. This is followed by long range diffusion when the tetragonal single phase decomposes to yield the equilibrium phases. If the initial lattice rearrangement occurs by congruent ordering, an irregular chessboard is eventually produced from the initial tweed structure, as shown in Figure 1.22 (B). Initial lattice rearrangement by displacive transformation produces tweed which develops into (110) polytwinned structure containing lamellae of two orientation variants of the tetragonal phase separated by lamellae of the equilibrium cubic phase, as shown in Figure 1.22 (C).

Thus, we can conclude that while tweed is a precursor for the chessboard structure, it may also be produced in other cases. The pseudospinodal decomposition is initiated in the vicinity of the crossover and leads to the development of the nanochessboard structure for a composition where the free energy of the cubic phase is slightly lower than that of the tetragonal phase. 

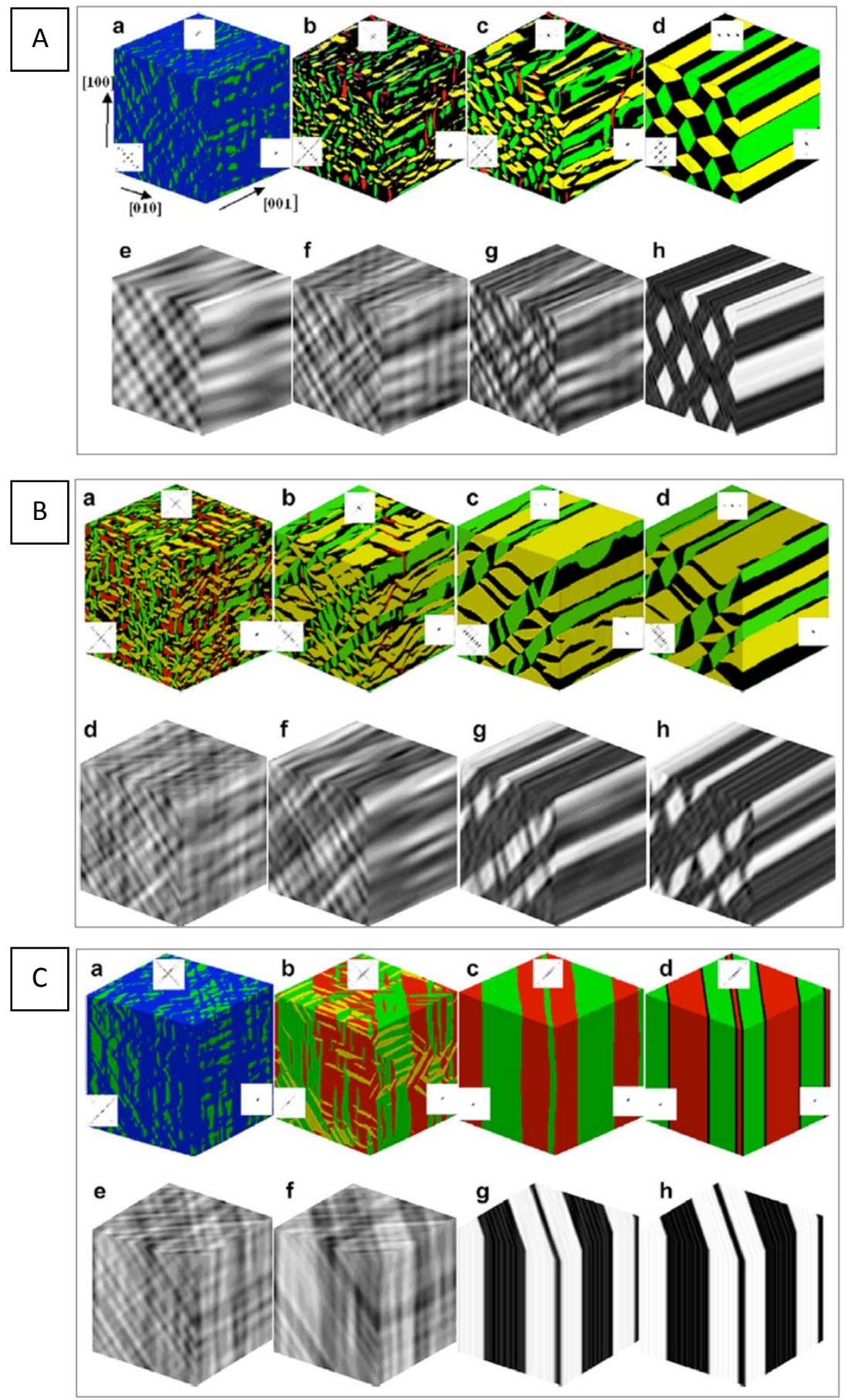

Figure 1.22: 3-D modeling of microstructure evolution involving disorder $\rightarrow$ order transformation for $(\mathrm{A}) \mathrm{c}^{\prime}<\mathrm{c}_{\mathrm{o}}$, (B) $\mathrm{c}^{\prime}>\mathrm{c}_{\mathrm{o}}$ and initiated by ordering and $(\mathrm{C}) \mathrm{c}^{\prime}>\mathrm{c}_{\mathrm{o}}$ and initiated by displacive transformation. In the upper (i.e., color) images, black represents the cubic phase while yellow, green and red respectively represent the $\mathrm{X}, \mathrm{Y}$ and $\mathrm{Z}$ variants of the tetragonal 
phase. The upper images in (A), (B), C) were processed to reveal more information obscured by perturbations to give the lower images. ${ }^{28}$

The above analysis by $\mathrm{Ni}$ and Khachaturyan ${ }^{28}$ can be extended to the eutectoid transformation in Co-Pt: $\underline{\mathrm{A} 1 \text { ( FCC ) } \rightarrow \mathrm{L}_{1}}+\underline{\mathrm{L}_{1}} \underline{2}$, which occurs by pseudospinodal decomposition and leads to the chessboard structure. The three-phase equilibrium is schematically illustrated in Figure 1.23 (a) for a slightly hypoeutectoid composition C'at the eutectoid temperature. It may be noted that the crossover refers to the intersection of the free energy curves of the $\mathrm{L}_{0}$ and $\mathrm{A} 1$ phases. Also, the composition is favorable for the pseudospinodal reaction, i.e., such that the free energy of the cubic phase is slightly lower than that of the tetragonal phase.

Let us assume that the compositions corresponding to minima in the $\mathrm{L} 1_{0}, \mathrm{~A} 1$ and $\mathrm{L}_{2}$ free energy curves, respectively $C_{L 10}, C_{A 1}$ and $C_{L 12}$, are constant for $T=T_{1}, T_{2}, T_{3}$ and $T_{4}$, where $T_{1}>T_{2}>T_{3}$ $>\mathrm{T}_{4}$. At $\mathrm{T}=\mathrm{T}_{1}$, the tetragonal phase is nucleated with non-equilibrium composition $\left(\mathrm{C}_{\mathrm{L} 10}<\mathrm{c}^{\prime}<\right.$ $\mathrm{C}^{\prime}$ ) indicated by the red tie line in Figure 1.23 (a). [Note that tweed could start forming at $\mathrm{T}>$

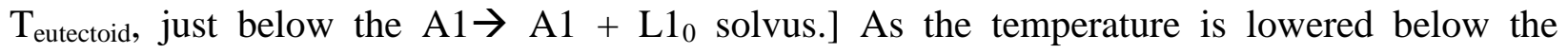
eutectoid and as the transformation proceeds, the compositions of the $\mathrm{L}_{0}$ precipitate and FCC matrix change simultaneously and continuously - towards $\mathrm{C}_{\mathrm{L} 10}$ in case of the precipitates, and towards and beyond $\mathrm{C}_{\mathrm{A} 1}$ for the matrix. This is illustrated in Figures 1.23 (b) $-(\mathrm{d})$. When the transformation is at the stage illustrated in Figure $1.23(\mathrm{~d})$, the composition of the $\mathrm{L} 1_{0}$ phase may approach its equilibrium value. However, the matrix phase is still FCC, now energetically unfavorable relative to the $\mathrm{L}_{2}$ phase at that composition, $\mathrm{C}_{\mathrm{L} 12}^{\prime}$. The matrix, then, undergoes congruent ordering to form the $\mathrm{L}_{2}$ phase with a composition $\mathrm{C}_{\mathrm{L} 12}^{\prime}$. Hereafter, the compositions of the $\mathrm{L}_{0}$ precipitates and $\mathrm{L}_{2}$ matrix continue to change simultaneously and continuously towards their equilibrium compositions - $\mathrm{C}_{\mathrm{L} 10}$ in case of the precipitate, and $\mathrm{C}_{\mathrm{L} 12}$ for the matrix. 

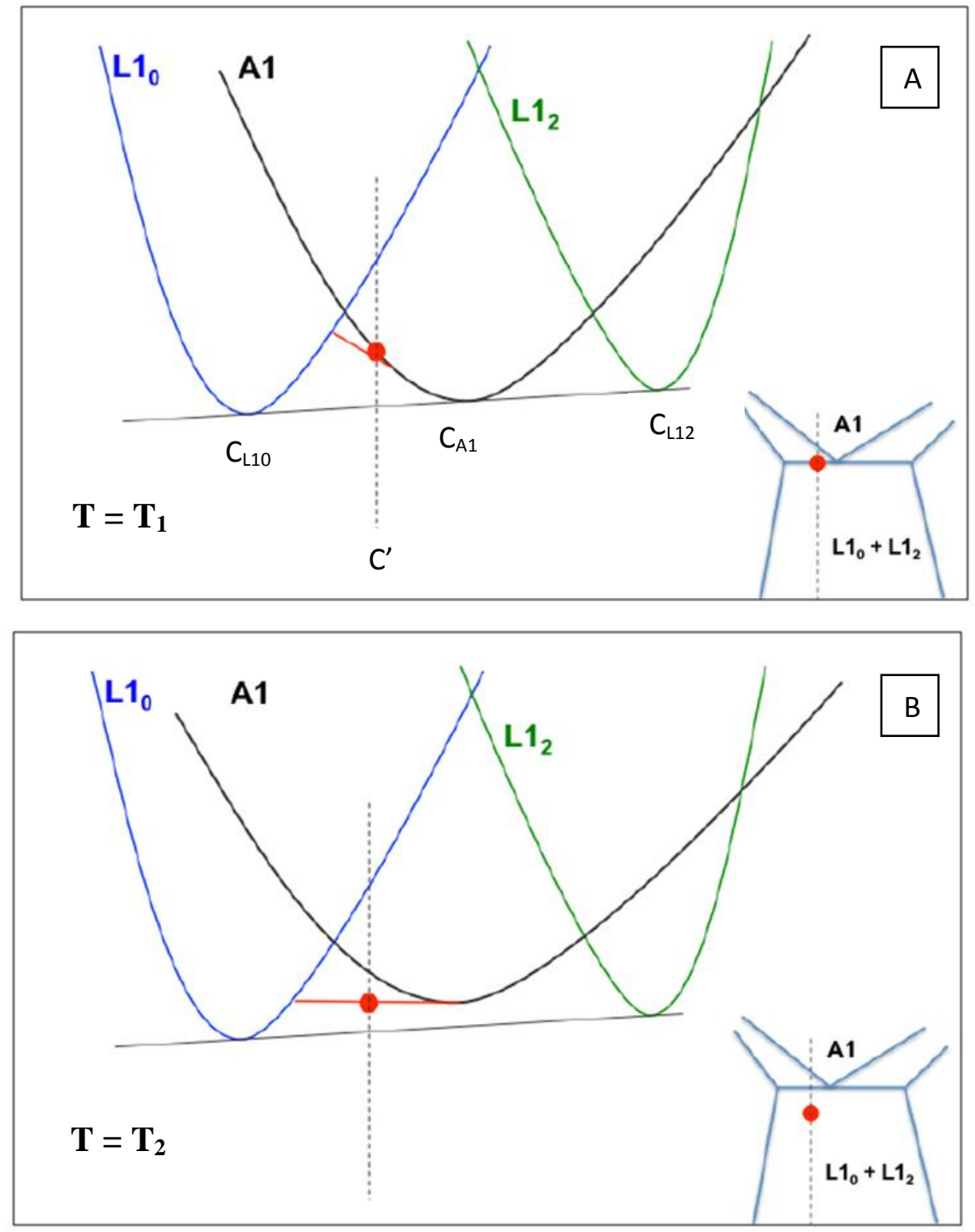

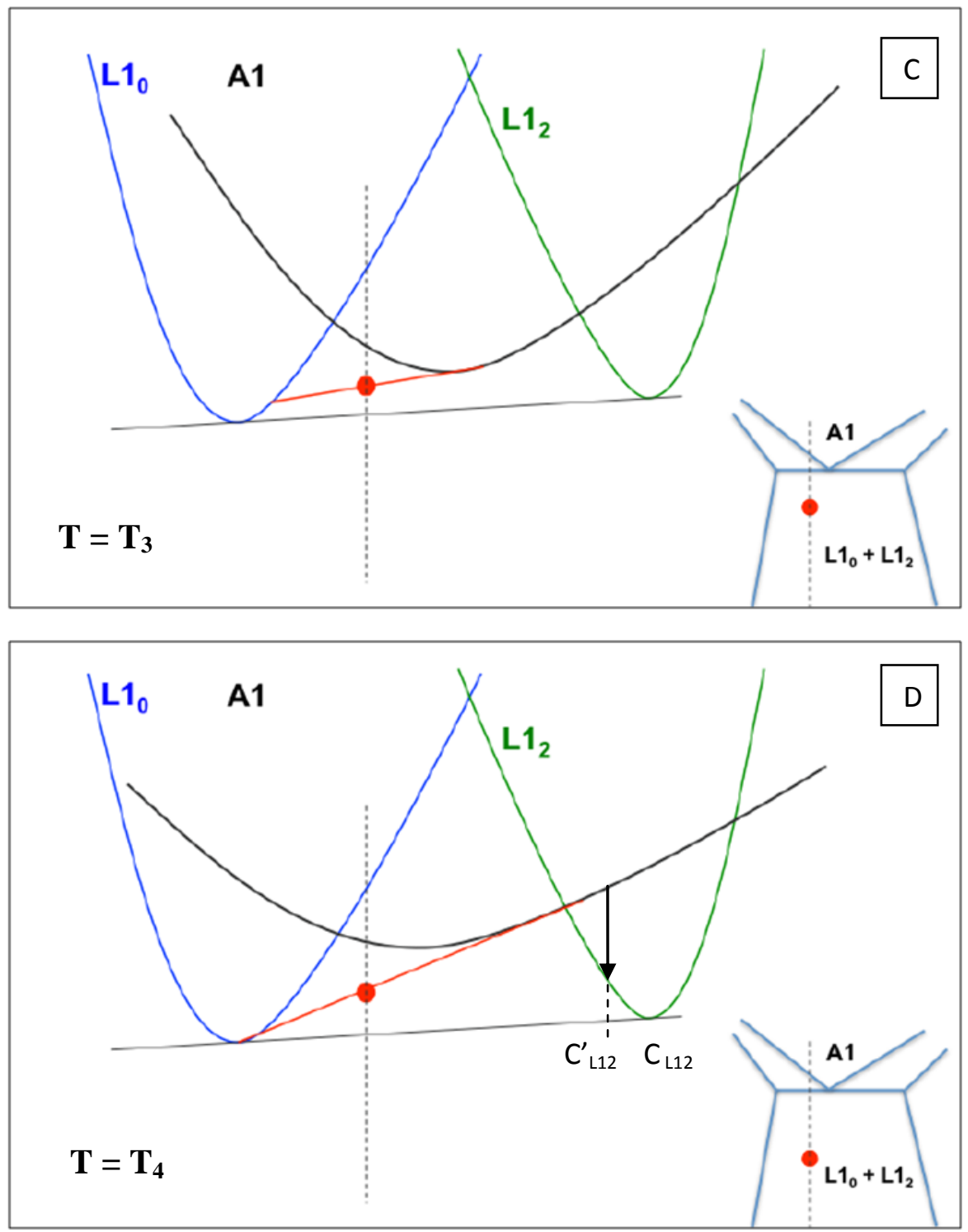

Figure 1.23: Schematic free energy curves for an alloy undergoing the eutectoid transformation. 


\subsection{Magnetic Behavior}

\subsubsection{Magnetic properties}

The schematic hysteresis loop in Figure 1.24 shows the variation of the magnetic moment per unit volume of a sample i.e., its magnetization $\mathrm{M}$, with the applied external magnetic field $\mathrm{H}$. The magnetic flux density, $\mathrm{B}=\mathrm{H}+4 \pi \mathrm{M}$, in CGS units. As an initially demagnetized sample is subjected to an increasing external magnetic field, its magnetization increases until the sample becomes saturated at $\mathrm{M}=\mathrm{M}_{\mathrm{s}}$; this portion of the figure is called the initial magnetization curve. When the applied field is then reduced to zero, the residual magnetization is called remanence, $\mathrm{M}_{\mathrm{r}}$. The reverse field required to reduce the magnetization to zero is called the intrinsic coercivity, $\mathrm{H}_{\mathrm{ci}}$, whereas the reverse field required to reduce $\mathrm{B}$ to zero is called the coercivity $\mathrm{H}_{\mathrm{c}}$. In the forthcoming chapters, however, we will use $\mathrm{H}_{\mathrm{c}}$ to denote $\mathrm{H}_{\mathrm{ci}}$. Further increasing the reverse field leads to saturation in the reverse direction; applying an increasing field in the original direction leads eventually to saturation in that direction, as shown in the hysteresis loop below.

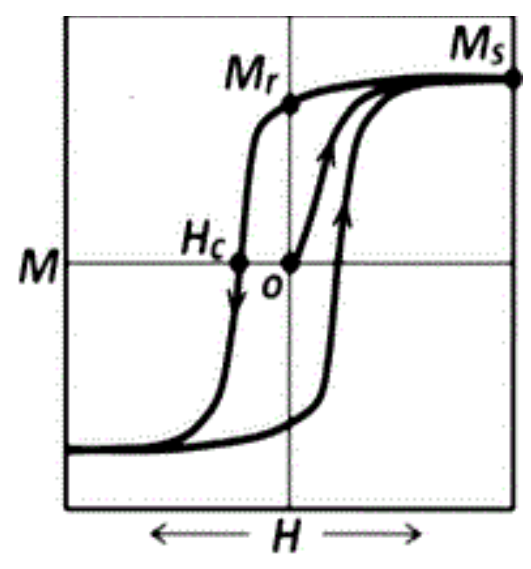

Figure 1.24: Schematic M-H hysteresis loop. ${ }^{30}$

The free poles of a magnet lead to a demagnetizing field $\mathrm{H}_{\mathrm{d}}$, which is the internal field produced in a magnetized sample in a direction opposite to that of its magnetization, resulting in a flux density lower than the remanent induction $\mathrm{B}_{\mathrm{r}}$. Consider a permanent magnet which was 
magnetized using a strong field. The actual magnetization after withdrawal of the applied field is indicated by point $\mathrm{P}$, as shown in Figure 1.25. This actual operating point $\mathrm{P}$ occurs at the intersection of the load line OC, whose slope depends on the geometry of the magnet, and the B$\mathrm{H}$ curve in the second quadrant, i.e., the demagnetization curve. The usefulness of the magnet is thus determined by the remnant induction, $\mathrm{B}_{\mathrm{r}}$, the shape of the demagnetization curve and the demagnetization field (and hence, the geometry of the sample). For a magnet of any volume, the energy stored in the magnetic field is proportional to $\mathrm{BH}$, which is called the energy product. The area $\mathrm{B}_{\mathrm{d}} * \mathrm{H}_{\mathrm{d}}$ enclosed by the rectangle $\mathrm{P}-\mathrm{H}_{\mathrm{d}}-\mathrm{O}-\mathrm{B}_{\mathrm{d}}$ in Figure 1.25 indicates the energy product for a magnet operating at point $\mathrm{P}$. The value of $\mathrm{BH}$ varies over the demagnetization curve, as shown in Figure 1.26. The highest efficiency of the magnet corresponds to the situation where the load line passes through the point corresponding to the maximum value of $\mathrm{BH}, \mathrm{BH}_{\max } \cdot{ }^{10,31}$

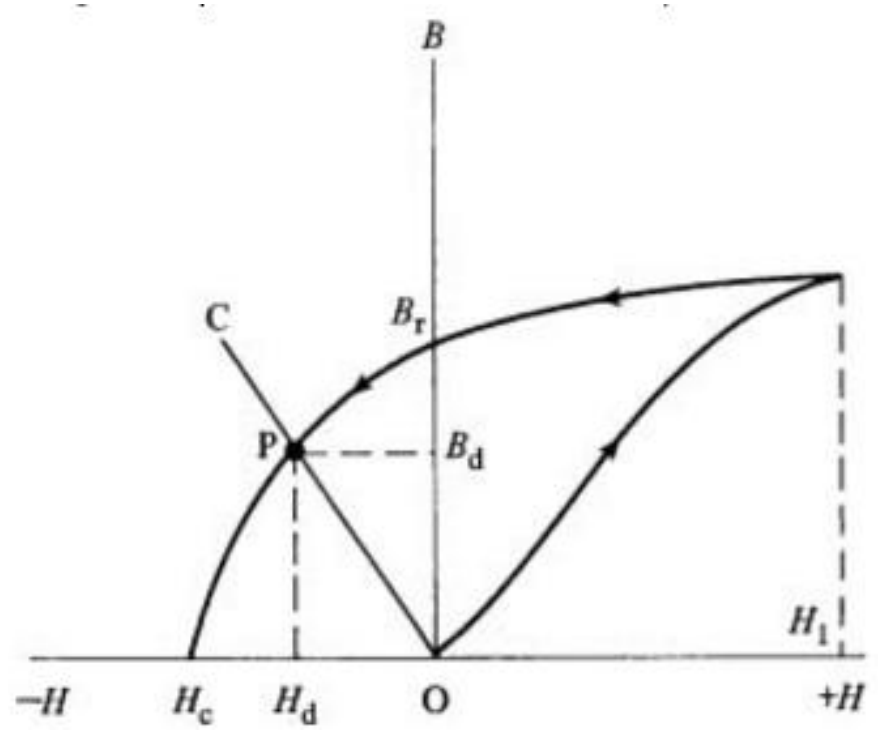

Figure 1.25: Initial magnetization, demagnetization curves for a permanent magnet and the operating point $\mathrm{P}^{31}$ 


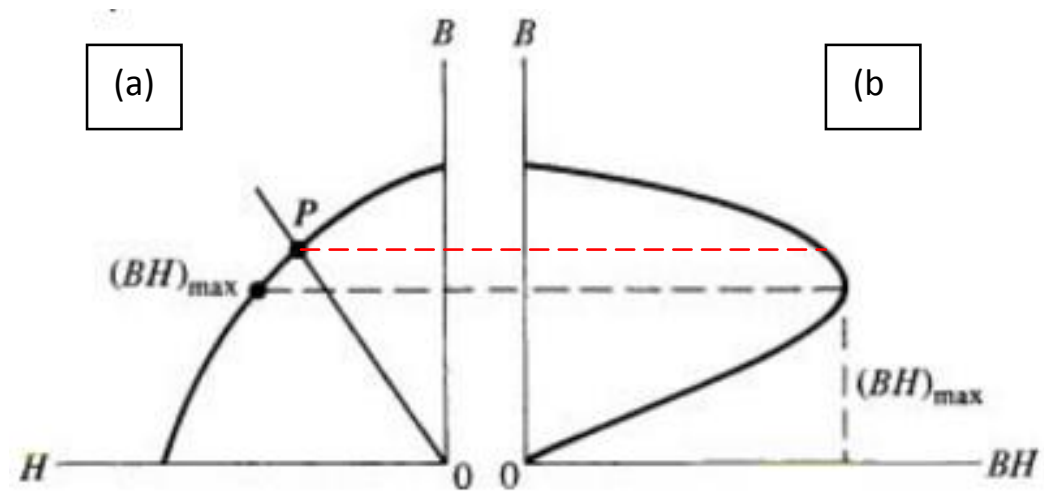

Figure 1.26: (a) Demagnetization curve and (b) variation of the energy product based on location of the operating point. ${ }^{31}$

\subsubsection{Domains and domain walls}

Domains are regions containing parallel magnetic moments and the interfaces between adjacent domains are called domain walls. In the demagnetized state, the vector sum of the magnetizations in all domains in the sample is zero. The process of magnetization progressively leads to the parallel alignment of all magnetization vectors within the sample. Ferromagnetic materials have associated magnetostatic, exchange, magnetocrystalline and magnetostrictive energies. Magnetostatic energy is associated with the demagnetizing field of a sample. As shown in Figure 1.27, this demagnetizing field, and consequently, the magnetostatic energy can be reduced by the formation of domains.

Multiple (versus single) domains are formed if this results in a decrease in the total energy of the ferromagnetic material. In other words, a particle/grain needs to be large enough to support a domain wall such that the interfacial energy gained is less than the magnetostatic energy reduced. The exchange energy is minimized by the alignment of electron spins and hence, magnetic moments. On account of the magnetocrystalline anisotropy, it is energetically favorable for the magnetization in a crystal to be aligned along a preferential crystallographic direction. Thus, the domains prefer to be oriented along the easy axis.

Domain walls represent regions of increased exchange energy on account of the non - parallel alignment of magnetic moments across the boundary. Due to exchange energy, the direction of 
magnetization varies gradually between two domains, i.e., the wall contains a range of orientations transitioning from the direction in one domain to another, in order to minimize the misorientation of spins. This would motivate infinitely large domain walls were it not for the fact that magentocrystalline energy is minimized if the moments are parallel to the easy axis and thus the magnetcrystalline anisotropy works to minimize the domain wall width. The actual width depends on a balance between the exchange and magnetocrystalline energies. The magnetization rotates within the plane of the wall, i.e., about the domain wall normal, in case of Bloch walls and in a plane perpendicular to the domain wall in case of Néel walls. ${ }^{32}$ Magnetostriction refers to the change in length (strain) of the sample that accompanies magnetization and the associated energy influences the lengths/sizes of the domains illustrated in Figure 1.27. ${ }^{33}$

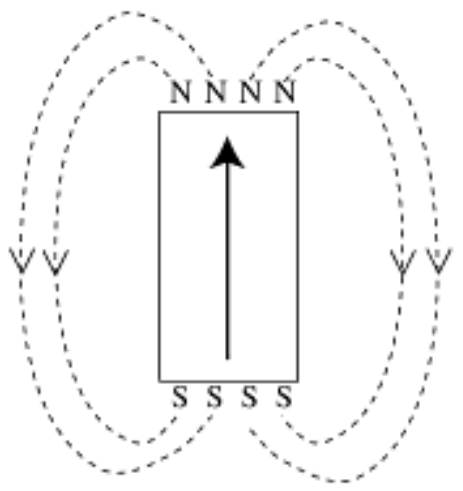

(a)

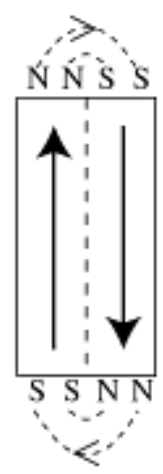

(b)

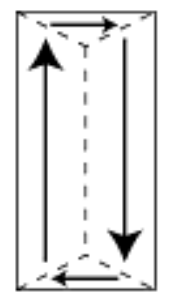

(c)

Figure 1.27: Domain formation in a ferromagnetic material leading to a reduction in the magnetostatic free energy. ${ }^{33}$

\subsubsection{Magnetization and magnetization reversal}

In particles/ large enough to support domain walls, magnetization and magnetization reversal proceed by domain wall motion. With the application of a magnetic field, the domains whose magnetization vectors are closest to the direction of the applied external field begin growing at the expense of other domains, by domain wall motion. The migrating domain wall may encounter crystal defects or regions having different magnetic properties, which can pin the wall if the defect or region represents a local energy minimum. Additional energy, provided by the 
external magnetic field, is required to unpin the domain wall. At a suitably high value of the external field, domain walls are eliminated and the sample consists of one domain; its magnetization lies along the easy axis closest to the direction of the applied field. A higher external field can help attain higher magnetization by rotating the magnetization parallel to its direction; the magnet is saturated at this stage. This describes the process of initial magnetization of the demagnetized sample. ${ }^{33}$

With removal of the magnetic field, the magnetization first rotates back to the easy axis. Thereafter, domains with reverse magnetization nucleate and grow under the influence of the demagnetizing field within the sample, resulting in partial demagnetization. The sample still possesses residual magnetization owing to the fact that the domain walls are unable to regain their original positions, since, unlike the applied-field driven magnetization described above, demagnetization depends on the demagnetizing field which is not strong enough to unpin domain walls from imperfections in the crystals. An additional field is needed in the reverse direction to reduce the magnetization to zero. ${ }^{33}$

As noted above, the motion of domain walls is hampered by crystal imperfections. These may be inclusions, i.e., regions with different spontaneous magnetization behavior compared to the rest of the crystal, for e.g., second phase particles, impurities, cracks, holes; or residual microstress. Interaction of domain walls with inclusions results in a reduction of the domain wall area and hence, energy. Furthermore, the intersection of a domain wall with an inclusion results in the redistribution of free poles and an accompanying decrease in the magnetostatic energy associated with the inclusion, as illustrated in Figure 1.28. The reduction in magnetostatic energy is much more significant compared to the reduction in domain wall energy. ${ }^{31}$ The presence of impurities may interact with the domain walls, and hence, the material's purity plays a role in the hysteresis behavior. A sample with many crystal defects will saturate at a higher field and require a higher reverse field to reduce magnetization to zero. A sample which is pure and has fewer crystal defects shows less resistance to magnetization as well as demagnetization. ${ }^{33}$

Residual microstress arises from crystal imperfections like dislocations and varies within the lengthscale of the grain diameter. When a $90^{\circ}$ wall interacts with the field associated with this residual microstress, the elastic distortion associated with magnetostriction in these walls interacts with the stress distribution associated with the imperfection. The effect of residual stress 
is to decrease the energy associated with magntostrictive strain and hence, the interaction results in a minimum in the magnetoelastic energy associated with the domain wall. No magnetostriction/ magnetostrictive strain is associated with a $180^{\circ}$ wall and the residual microstress results in a decrease in the energy of an interacting domain wall by introducing stress anisotropy. ${ }^{31}$

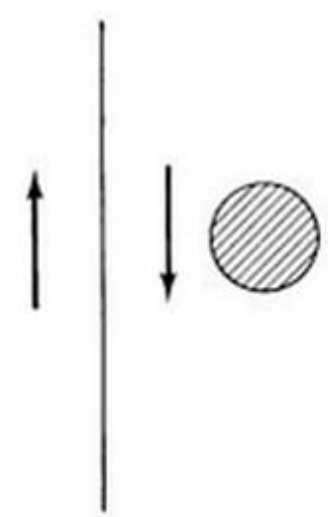

(a)

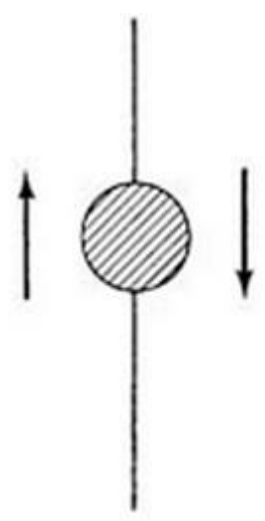

(b)

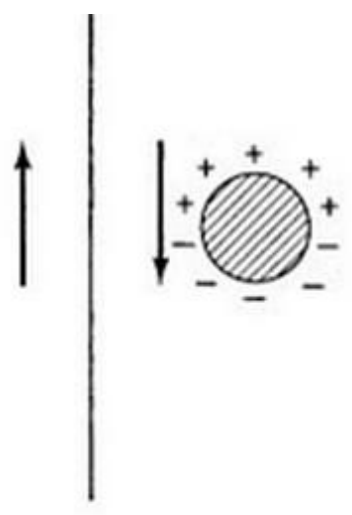

(c)

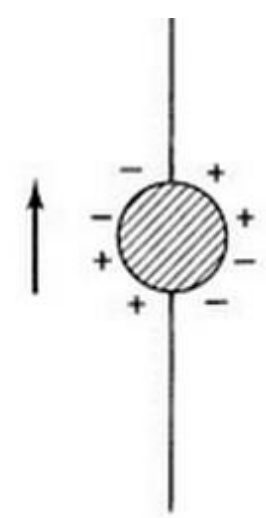

(d)

Figure 1.28: Interaction between a domain wall and an inclusion. ${ }^{31}$

Residual microstress and local changes in magnetic properties may also arise from APBs, a large number of which would occur within the twin plates of the polytwinned structures. Two types of magnetic domain walls may be identified in this microstructure: immobile or frozen domain walls which coincide with macrotwin boundaries and mobile serrated/kinked 180-degree domain walls which occur with macrotwin plates, cutting across the contained microtwin plates. ${ }^{22,34}$ This is illustrated in the micrograph presented in Figure 1.29 (a) and its schematic representation in Figure 1.29 (b). The high density of APBs in the microtwins leads to weak pinning of the mobile domain walls, which is proposed to be the controlling factor in magnetization reversal of alloys with the polytwinned structure. ${ }^{23,35}$ 


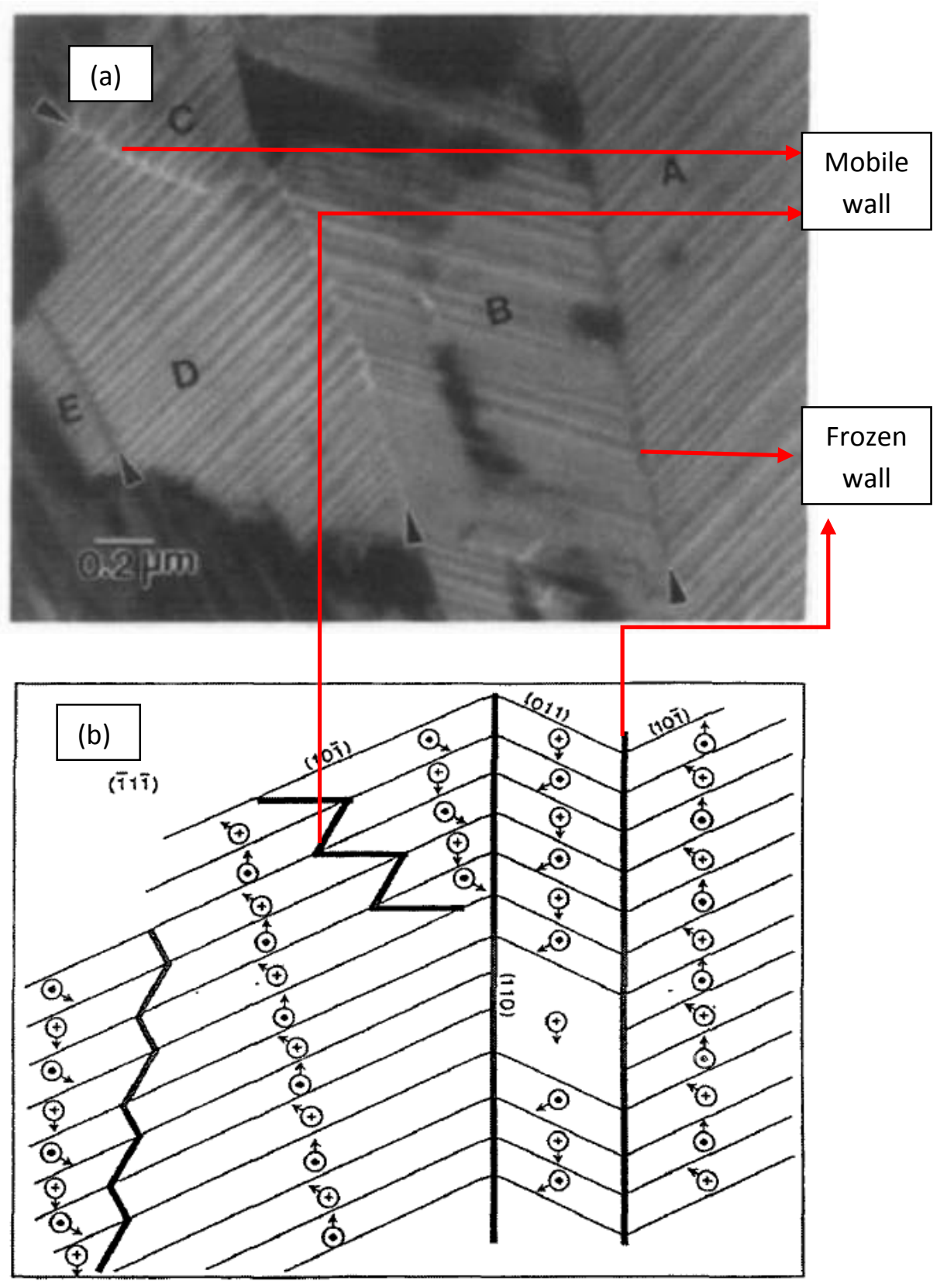

Figure 1.29: (a) Fresnel underfocus image revealing microtwins, macrotwins and domain walls in Fe-Pd. ${ }^{30}$ (b) Schematic representation of (a). ${ }^{19}$ 
Particles smaller than a critical minimum size contain a single domain, i.e., no domain walls. As discussed above, the contributions from domain wall energy and the magnetostatic energy help determine whether it is energetically favorable for a particle to exist as a single domain or multidomain particle. These energies depend on the particle radius: the domain wall energy varies as $r^{2}$ and the magnetostatic energy varies as $r^{3}$. In single domain particles, the magnetization lies parallel to the crystallographically easy axis. If an external field is applied parallel to the easy axis but along a direction opposite to that of the magnetization, magnetization reversal proceeds via rotation through the hard direction to the new easy direction (domain wall motion is not an option for the particle). This is schematically illustrated in Figure 1.30 (a) and the corresponding M-H loop is shown in Figure 1.30 (b). If the field is applied along a hard direction, the magnetization is gradually rotated into the hard direction of the field from the easy direction, in opposition to the anisotropy, but when the field is withdrawn, the magnetization rotates back to the easy direction. There is no hysteresis. ${ }^{33}$ The magnetization behavior and M-H loops for this case are shown in Figure 1.30 (c) and (d) respectively. If the spins of all atoms in the particle remain parallel to each other during rotation, as in Figure 1.30, the reversal mechanism is called coherent rotation and is associated with a coercivity of $2 \mathrm{~K} / \mathrm{M}_{\mathrm{s}}$, $\mathrm{K}$ being the magnetocrystalline anisotropy constant. If all spins do not remain parallel during rotation, the mechanism is called incoherent rotation. ${ }^{31}$ 


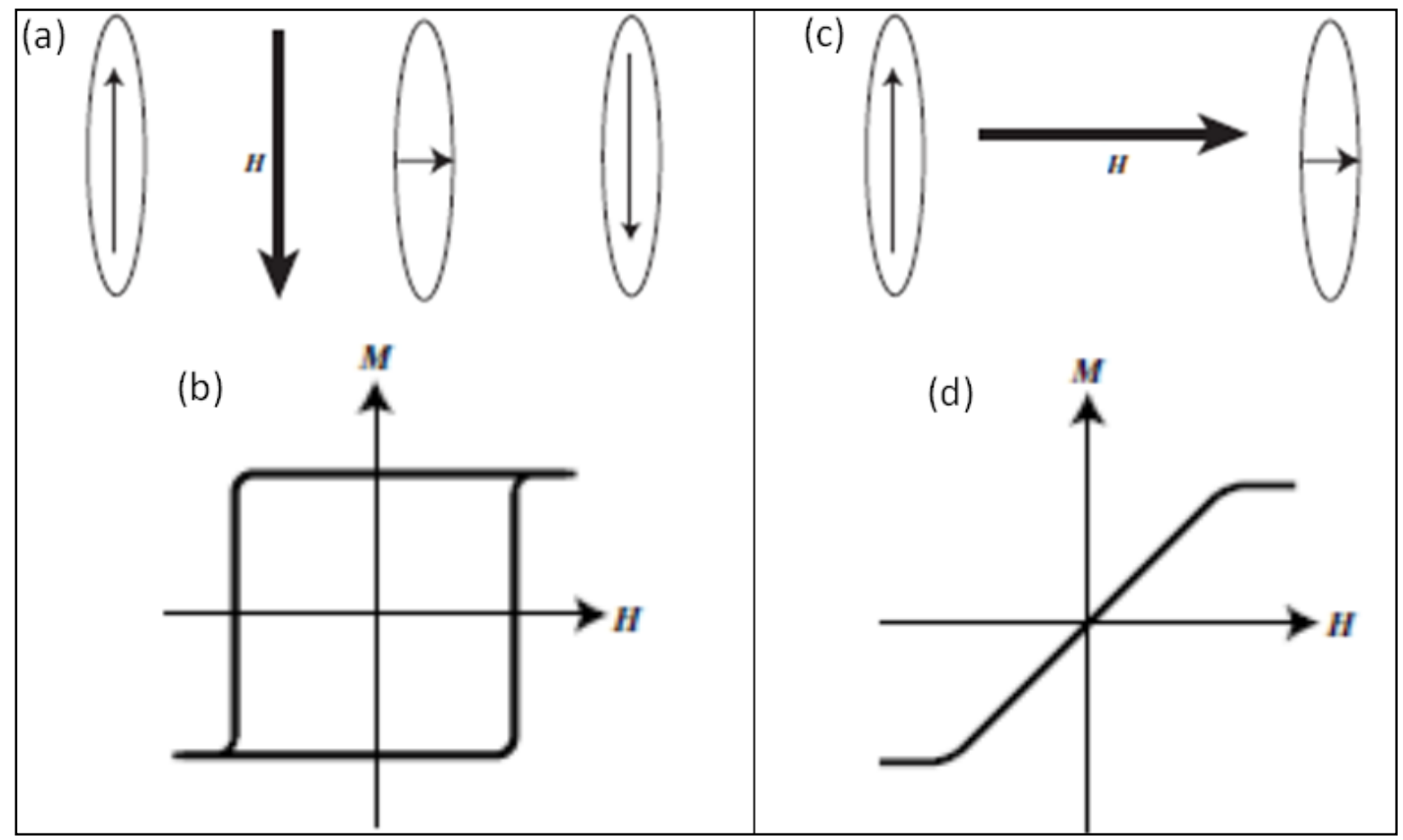

Figure 1.30: (a) Magnetization mechanism and (b) $\mathrm{M}-\mathrm{H}$ behavior when applied external field is parallel to an easy direction. (c) Magnetization mechanism and (d) M-H behavior when applied external field is along a hard direction.

\subsubsection{Exchange coupling and exchange spring behavior}

The Ising model describes ferromagnetism on the basis of the interaction between atoms in terms of an interaction energy $E_{\text {in }}$, as:

$E_{\text {in }}=-J \Sigma_{<i j>} S_{i} S_{j}-H \Sigma_{i} S_{i}$

Here, the spins are denoted as $\mathrm{S}_{\mathrm{i}}, \mathrm{s}_{\mathrm{j}}$, being 1 for spin up and -1 for spin down orientations; $\mathrm{H}$ represents the external magnetic field and $\mathrm{J}$, the interaction strength. $\mathrm{J}>0$ in ferromagnetic materials as the total energy is minimized if spins $\mathrm{S}_{\mathrm{i}}, \mathrm{S}_{\mathrm{j}}$ are oriented in the same direction. $\mathrm{J}<0$ in antiferromagnetic materials as the total energy is minimized if spins $S_{i}, S_{j}$ are oriented in opposite directions. $^{36}$ 
Ferromagnets with exchange-coupled two-phase microstructures can behave as a magnetic single phase. The soft and hard phases will reverse independently in the absence of inter-phase exchange coupling, for instance, if the size of the soft phase is sufficiently large. Factors governing exchange coupling include the magnetic properties, volume fractions, size and distribution and the nature of the interface between the constituent phases. ${ }^{37}$

In an exchange-coupled two-phase nanocomposite, the two phases - one with hard and the other with soft ferromagnetic properties - must be distributed on a fine scale. Coherent interphase interfaces help improve the coupling. In the Co-Pt eutectoid system, the hard $\mathrm{L}_{0}$ and soft $\mathrm{L}_{2}$ phases emerge from a common matrix phase and have fine lengthscales and coherent interfaces. 38 Thus, this system has a good potential for the development of exchanged coupled nanocomposites by means of suitable thermal processing.

The hysteresis loops for the single phase hard, soft materials and for the exchanged coupled composite comprising of these hard and soft phases are schematically illustrated in Figure 1.31. The exchange coupling of the hard ferromagnetic $\mathrm{L}_{0}$ and soft ferromagnetic $\mathrm{L}_{2}$ phases ideally results in improved magnetic properties. The $\mathrm{L}_{2}$ phase enhances the remanence, owing to its high saturation, while the $\mathrm{L} 1_{0}$ phase results in a larger coercivity. Upon exchange coupling, the energy product of the two phase permanent magnets is predicted to be higher than that resulting from constituent phases. ${ }^{38,39}$ Remanence enhancement, a remanence to saturation ratio $\mathrm{M}_{\mathrm{r}} / \mathrm{M}_{\mathrm{s}} \geq$ 0.5 and single phase demagnetization behavior indicate exchange coupling. ${ }^{4,38}$

Exchange coupled nanocomposites of hard and soft magnets phases are called exchange spring magnets if characterized by reversible demagnetization curves/ steep recoil loops. ${ }^{4,} 37,38 \mathrm{~A}$ schematic illustration of hysteresis loops of optimally aged and overaged exchange spring magnets, conventional single phase ferromagnets and a mixture of hard and soft magnets which reverse independently is shown in Figure 1.32.

The model by Kneller and Hawig was the first to consider exchange spring behavior, and it represents a simple and accessible approach ${ }^{38}$. Although it is simplistic, especially since it is only a 1D model, it does help motivate key considerations of length scales relevant to exchangespring ferromagnetism. Also, this model is effectively a domain-wall-nucleation model, although most material systems it gets applied to reverse via rotation. 


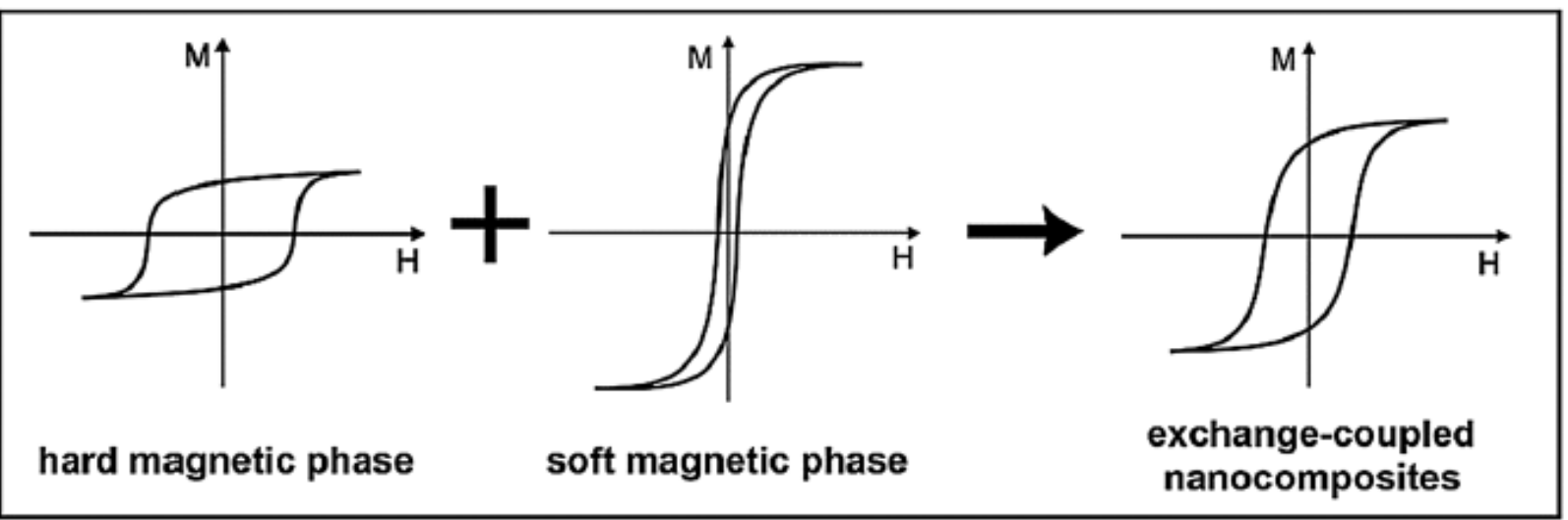

Figure 1.31: Schematic representation of hysteresis loops of single phase hard, soft magnets and an exchange-coupled nanocomposite of both these phases. $^{37}$

Consider an exchange coupled nanocomposite containing the hard ferromagnetic k-phase and soft ferromagnetic m-phase regions, of thickness $2 \boldsymbol{b}_{\boldsymbol{k}}$ and $2 \boldsymbol{b}_{\boldsymbol{m}}$ respectively, as shown in Figure 1.33. It is assumed that the hard k-phase is reasonably thick, about twice its equilibrium domain wall width $\left(\delta_{\mathrm{ok}}\right)$ :

$2 \mathrm{~b}_{\mathrm{k}} \approx 2 \delta_{\mathrm{ok}}=2 \pi\left(\mathrm{A}_{\mathrm{k}} / \mathrm{K}_{\mathrm{k}}\right)^{1 / 2}$,

where $A_{k}$ is the exchange constant and $K_{k}$ the magnetocrystalline anispotropy of the hard kphase. The state of saturation remanence where the magnetizations of both phases are parallel to the easy direction is shown in Figure 1.33(a). The magnetization begins to change reversibly in the soft phase upon application of an increasing reverse field. If the soft phase thickness is large, say, equal to twice its equilibrium domain wall thickness $\left(\delta_{\mathrm{om}}\right)$ :

$2 \mathrm{~b}_{\mathrm{m}} \approx 2 \delta_{\mathrm{om}}=2 \pi\left(\mathrm{A}_{\mathrm{m}} / \mathrm{K}_{\mathrm{m}}\right)^{1 / 2}$,

then, two equilibrium $180^{\circ}$ domain walls are formed reversibly in the m-phase, as shown in Figure 1. 33 (b). Here, $A_{m}$ is the exchange constant and $K_{m}$ the magnetocrystalline anispotropy of the soft m-phase. It may be noted that $\delta_{\mathrm{om}} \gg \delta_{\mathrm{ok}}$ since $\mathrm{K}_{\mathrm{m}}<<\mathrm{K}_{\mathrm{k}}$. When the reverse field is further increased, these domain walls in the soft m-phases are compressed reversibly towards the hard k-phase boundary, as shown in Figure 1.33 (c). Consequently, the energy density of these domain walls $\left(\mathrm{E}_{\gamma \mathrm{m}}=\gamma_{\mathrm{m}} / \delta_{\mathrm{m}}\right)$ becomes higher than the energy density of walls of equilibrium thickness $\left(\mathrm{E}_{\gamma \mathrm{om}}=\gamma_{\mathrm{om}} / \delta_{\mathrm{om}}\right)$. The magnetization in the hard k-phase continues unchanged. 


\section{EXCHANGE SPRING}

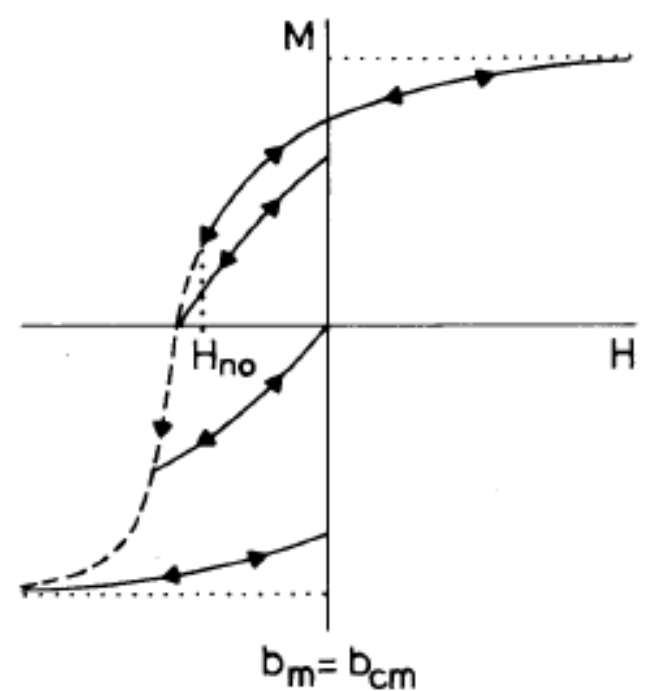

(a)

CONVENTIONAL

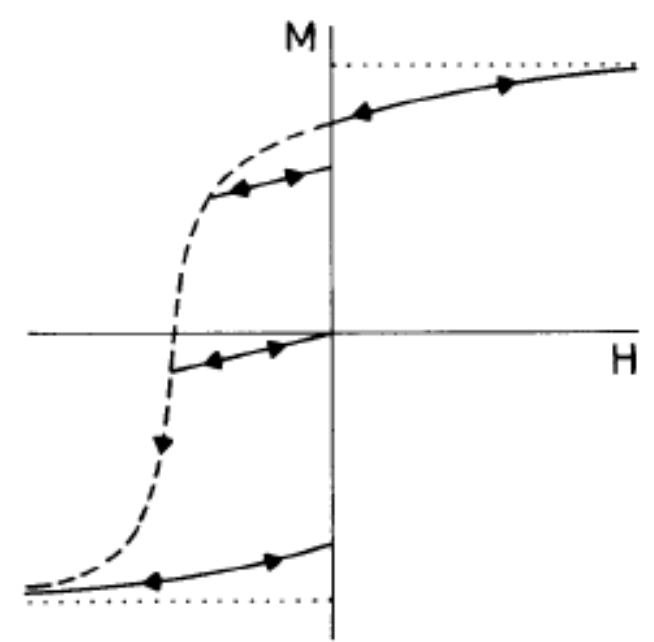

(c)

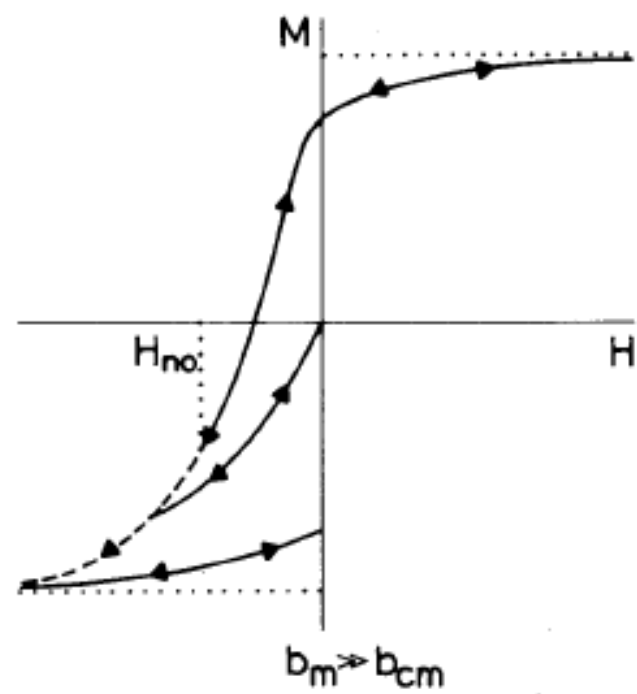

(b)

TWO INDEPENDENT PHASES

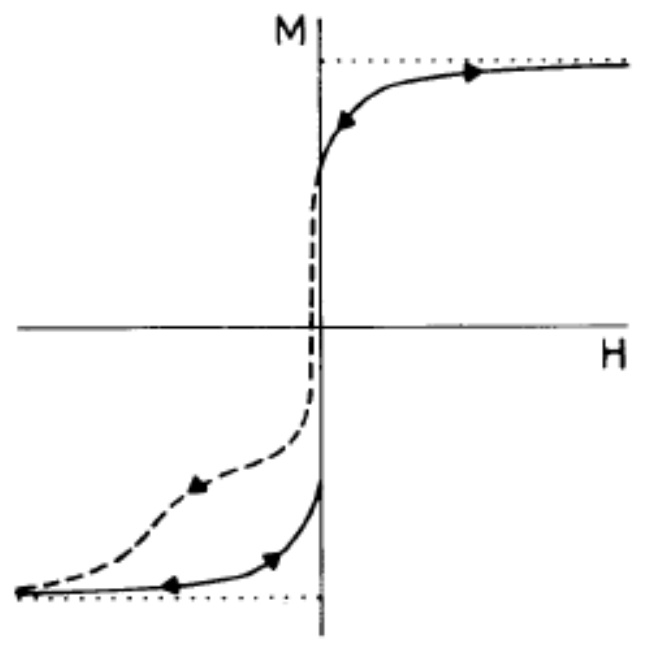

(d)

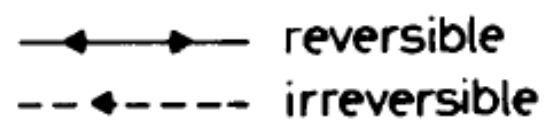

Figure 1.32: Schematic demagnetization curves in (a) exchanged coupled magnet with optimum microstructure resulting in superior magnetic properties compared to an (b) exchange coupled magnet with overaged microstructure; (c) a conventional single phase ferromagnet and (d) mixture of soft and hard ferromagnetic phases which switch independently. ${ }^{38}$ 
At a reverse field $\mathrm{H}=\mathrm{H}_{\mathrm{no}}$, the value of $\mathrm{E}_{\gamma \mathrm{m}}$ approaches the equilibrium energy density of a wall in the hard k-phase $\left(\mathrm{E}_{\gamma \mathrm{ok}}\right)$ :

$\mathrm{E}_{\gamma \mathrm{m}}=\gamma_{\mathrm{m}} / \delta_{\mathrm{m}} \approx \mathrm{E}_{\gamma \mathrm{ok}}=\gamma_{\mathrm{ok}} / \delta_{\mathrm{ok}}=\left[2 \pi\left(\mathrm{A}_{\mathrm{k}} \mathrm{K}_{\mathrm{k}}\right)^{1 / 2}\right] /\left[\pi\left(\mathrm{A}_{\mathrm{k}} / \mathrm{K}_{\mathrm{k}}\right)^{1 / 2}\right]=2 \mathrm{~K}_{\mathrm{k}}$.

At this point the domain wall in the soft m-phase moves into the hard k-phase, resulting in irreversible magnetization reversal of both phases, as shown in Figure 1.33 (d). $\mathrm{H}_{\mathrm{no}}$ is lower than the field required for coherent rotation of the hard phase, $\mathrm{H}_{\mathrm{Ak}}=2 \mathrm{~K}_{\mathrm{k}} / \mathrm{M}_{\mathrm{sk}}$, but of the same order of magnitude. ${ }^{38}$ Thus, the magnetization reversal is reversible until the attainment of $\mathrm{H}_{\text {no }}$.

Kneller and Hewig ${ }^{38}$ have established the optimum microstructure for an exchange spring magnet, wherein the spacing and size of the hard phase particles are nearly equal and equal to about twice the equilibrium domain width of the hard phase, $\approx 10 \mathrm{~nm}$, as indicated in Figure 1.34. This corresponds to a composition of nearly $10 \%$ by volume of the hard phase, which is distributed homogeneously in the soft matrix. The soft phase particles are too small to support a domain wall and would therefore reverse by rotation, in the optimum microstructure. This optimum microstructure results in the highest coercivity and a saturation magnetization that is nearly equal to that of the soft phase.

Figure 1.35 compares the exchange coupling and exchange spring behavior in hard/soft ferromagnetic nanocomposites as a function of the soft phase dimension. For the case shown in Figure 1.35 (a), the soft phase dimension is larger than twice the correlation length $\lambda_{c}$. When an external reverse magnetic field is applied, the hard phase resists magnetization reversal but the magnetization of the soft phase switches irreversibly. When the external magnetic field is removed, the magnetization of the soft phase does not switch back to its initial orientation. In this case, the hard and soft ferromagnetic phases are not exchange coupled and do not exhibit exchange spring behavior.

The situation where the soft phase dimension is smaller than twice the correlation length $\lambda_{c}$ is illustrated in Figure 1.35 (b). The magnetization of the hard phase does not rotate whereas the magnetization of the soft phase rotates reversibly in response to an external reverse field. 
However, as soon as the field is removed, the hard phase enables the magnetization of the soft phase to return to its original orientation. Thus, for suitably small dimensions of the soft phase, the magnetizations of the hard and soft phases are coupled and the nanocomposites exhibits exchange spring behavior.

(a)

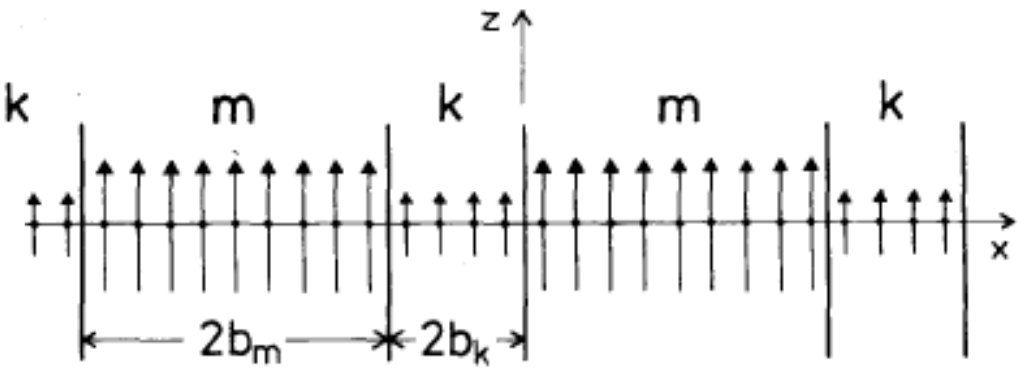

0

(b)
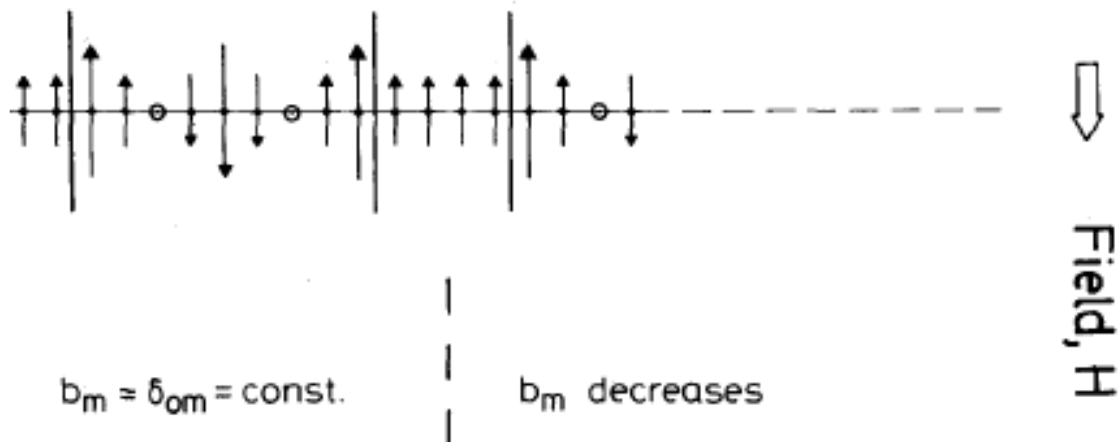

(c)

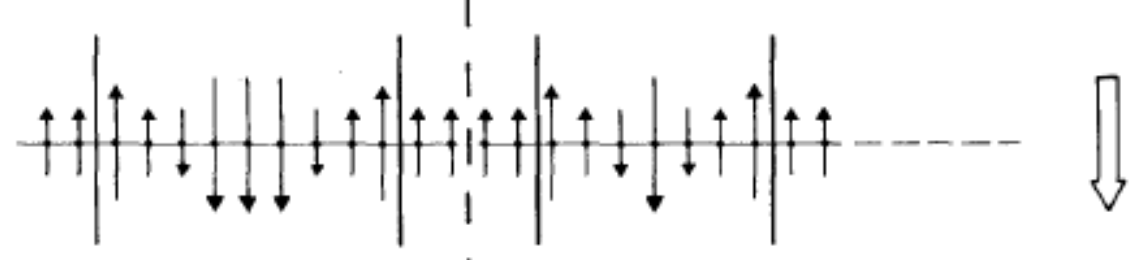

(d)

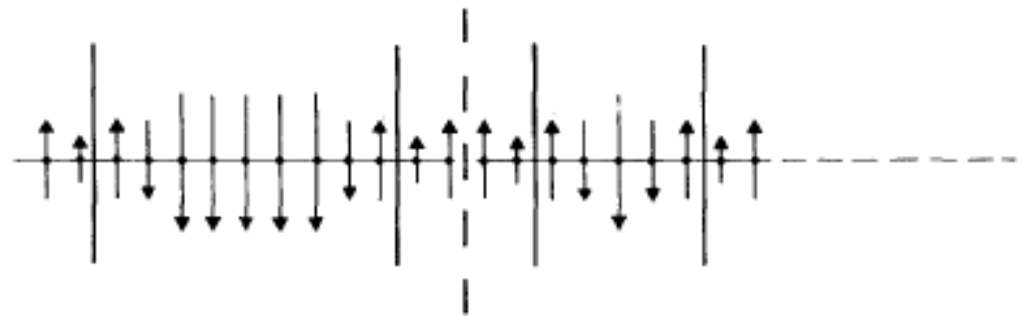

Figure 1.33: 1-D representation of the stages of magnetization reversal in an exchange-coupled composite showing (a) saturation remanence, (b) reversible formation of domain walls in soft $\mathrm{m}$ phase, (c) reversible compression of these domain walls towards the hard k-phase and (d) irreversible rotation in both phases. ${ }^{38}$ 


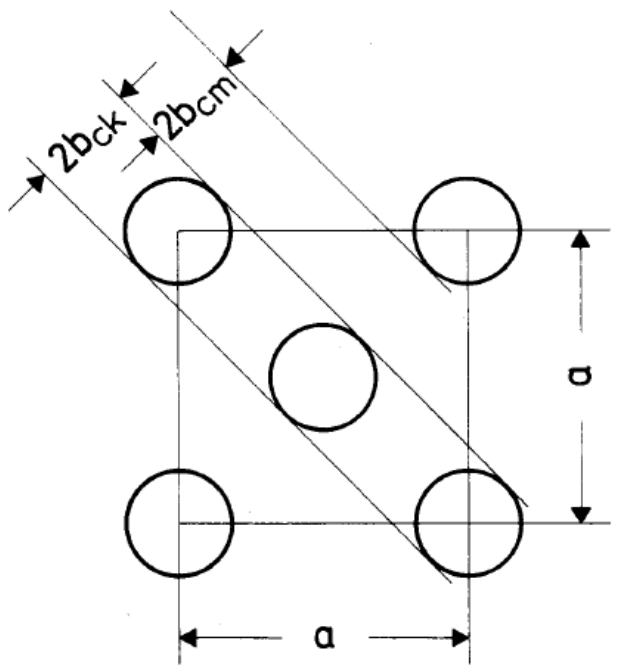

Figure 1.34: Schematic representation of the optimum microstructure in an exchange coupled magnet. $^{38}$

(a)

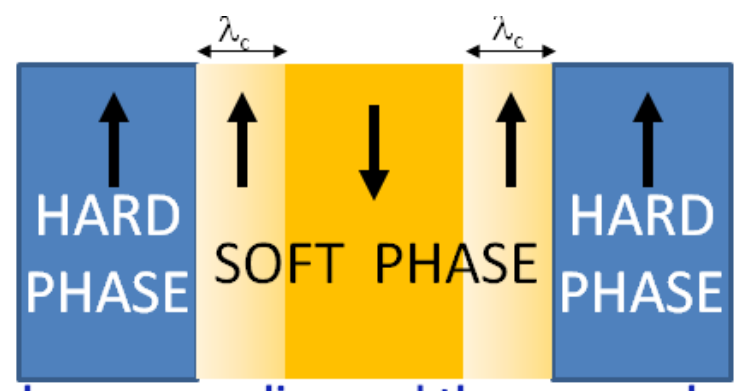

Large soft phase: No exchange coupling and thus, no exchange spring behavior

(b)

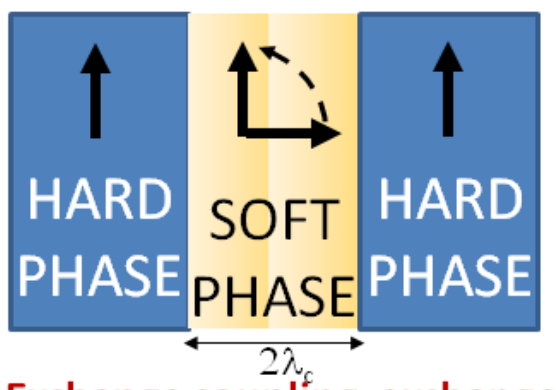

Optimum soft phase: Exchange coupling, exchange spring behavior

Figure 1.35: Exchange coupling, exchange spring behavior in a hard/soft nanocomposite as a function of soft phase dimension. 


\subsection{Research goals}

This research seeks to establish the interrelationships between processing, microstructure and magnetic properties in near-eutectoid Co-Pt alloys. We will attempt to reproduce the nanochessboard microstructure in Co-Pt alloys that undergo the eutectoid reaction. Relevant magnetic properties, exchange coupling, exchange spring behavior and magnetization reversal will be investigated in view of the phase transformation leading to the chessboard structure. Compositions marginally outside the eutectoid reaction isotherm will be examined to determine the individual behavior of the $\mathrm{L} 1_{0}$ and $\mathrm{L}_{2}$ phases in the vicinity of the two phase region.

Processing and characterization techniques are described in Chapter 2. Results pertaining to the processing - structure - property relationships in marginally off-eutectoid Co-Pt alloys are presented and analyzed in Chapter 3. Magnetic properties and their microstructural origins in CoPt alloys undergoing the eutectoid transformation are presented and discussed in Chapter 4. The results of our study are summarized, a comparison between the various compositions is presented and potential avenues for further research are outlined in Chapter 5. 


\section{Experimental Procedure}

The Co-Pt alloy compositions considered in this study were processed using electric arc melting. Following aging treatments, the magnetic properties and underlying microstructure of the alloys were investigated using X-Ray Diffraction, Vibrating Sample Magnetometry and Transmission Electron Microscopy. The techniques used for processing and characterizing the Co-Pt alloys are described below.

\subsection{Electric Arc Melting}

Bulk binary Co-Pt alloys were processed by arc melting high purity Co (99.9\%) and Pt (99.99\%) in an argon atmosphere. Pieces of Co and Pt were first cleaned by sonicating in an ethanol bath, dried and then carefully weighed out. The total weight of the 'charge' was measured, accurate to 3 decimal places. The charge was then transferred to an insert, which fits onto the hearth. Another smaller insert containing Ti slugs was also placed onto the hearth. The arc melting chamber was then closed and evacuated using roughing and diffusion pumps to a pressure of $5^{*} 10^{-6}$ torr. A few minutes before melting, the chamber was back-filled with Argon upto a partial pressure of 10 inches $\mathrm{Hg}$; the indicator in the sub-atmospheric pressure gauge indicated 20 inches $\mathrm{Hg}$.

An arc was initially struck on the Ti slugs using a tungsten 'stinger' with a sharp tip. First melting the 'getter', in this case $\mathrm{Ti}$, allows any residual oxygen in the chamber atmosphere to be captured. The arc was then moved onto the charge. The charge was melted down completely and continuously stirred with the help of the arc, until it became a sessile drop. The arc was then extinguished and the Co-Pt button allowed to solidify and cool. The button was then overturned with the help of the stinger. The melting process was repeated again. The sample was overturned

and melted two more times, to ensure homogeneity of the alloy. Upon complete cooling, the button was removed from the chamber. We have processed buttons ranging from $50-70 \mathrm{~g}$ in mass and $1.75-2.25$ inches in diameter. 


\subsection{Inductively Coupled Plasma - Optical Emission Spectroscopy (ICP-OES)}

This technique was employed to determine the composition of the Co-Pt alloys processed by arc melting. The analytes present in the solid samples must first be obtained in solution by acid digestion. This solution, when introduced into the ICP-OES apparatus, is converted into an aerosol. The aerosol quickly transforms to vapor state when directed into the core of the plasma where equivalent temperatures are as high as $10000 \mathrm{~K}$. In the gaseous states, the elements present in solution become available as free atoms. Furthermore, the energy from collisions within the plasma gives rise to atomic and ionic excited state species. Both kinds of species release photons of characteristic wavelengths when they relax to the ground state, thus enabling identification of elements. ${ }^{40}$

We dissolved $25 \mathrm{mg}$ samples of each alloy in $4 \mathrm{ml}$ hot aqua regia and analyzed them using the iCAP 6200 spectrometer. The compositions of the four different alloys we processed were determined to be: (1) $\mathrm{Co}_{41.7} \mathrm{Pt}_{58.3}$, (2) $\mathrm{Co}_{37.6} \mathrm{Pt}_{62.4}$ which lie on either side of the eutectoid isotherm and (3) $\mathrm{Co}_{40.5} \mathrm{Pt}_{59.5}$, (4) $\mathrm{Co}_{38.5} \mathrm{Pt}_{61.2}$ which lie on the eutectoid isotherm, as shown in Figure 2.1 below. Statistical analysis gave an error of \pm 0.2 atom $\%$ in the compositions determined using this technique. The calculations pertaining to this error analysis are included in Appendix 1. 


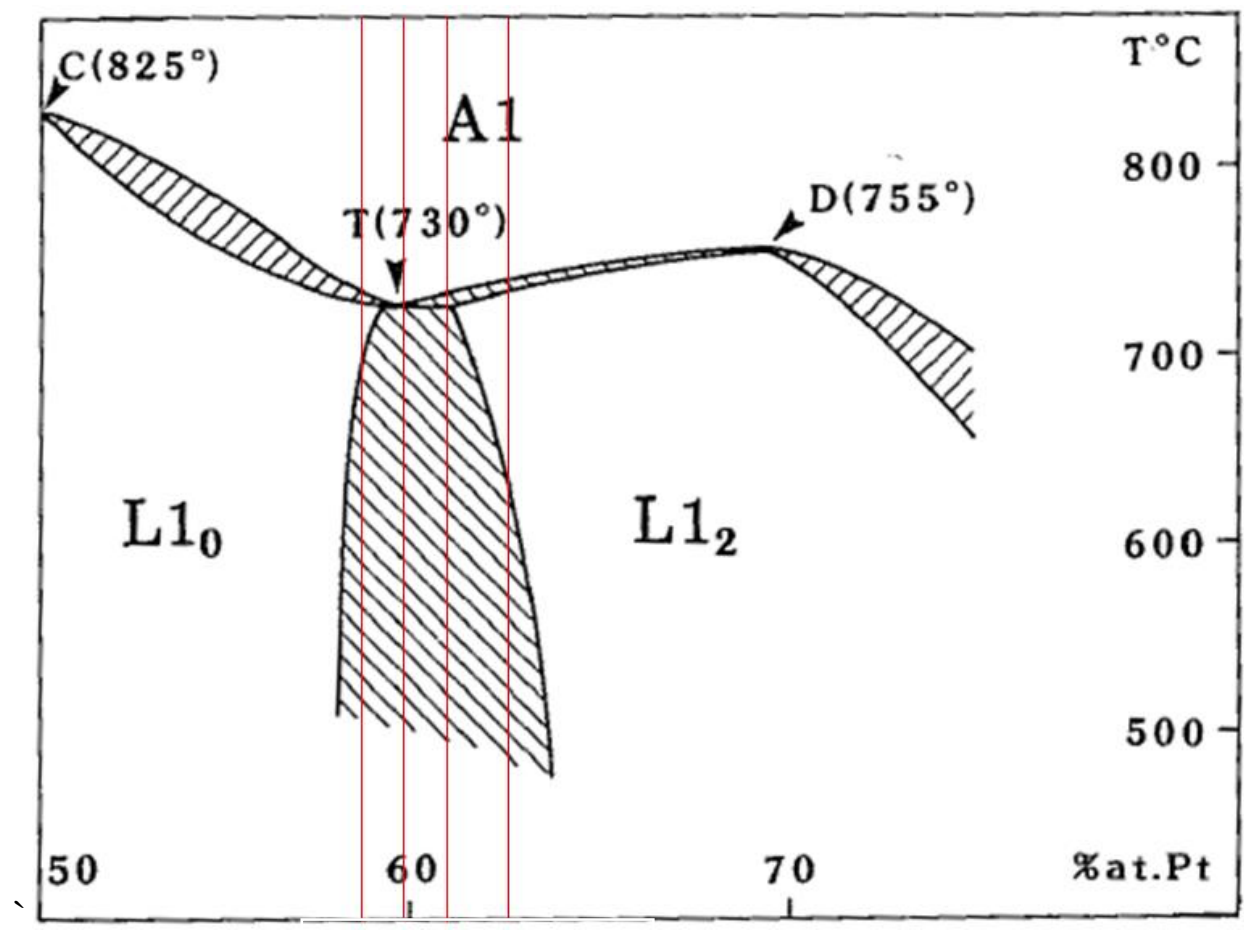

(1) (3)(4) (2)

Figure 2.1: Binary Co-Pt alloy compositions under consideration in this study: (1) $\mathrm{Co}_{41.7} \mathrm{Pt}_{58.3}$, (2) $\mathrm{Co}_{37.6} \mathrm{Pt}_{62.4}$ (3) $\mathrm{Co}_{40.5} \mathrm{Pt}_{59.5}$ and (4) $\mathrm{Co}_{38.5} \mathrm{Pt}_{61.2}$.

\subsection{Cold-Rolling}

The alloy buttons were subjected to $\approx 60 \%$ cold work over the course of several passes in the rolling mill. The highly cold-worked button was then cut into conveniently sized pieces using the Brilliant 220 cut-off saw. These pieces were then encapsulated and homogenized. Cold-rolling and homogenization/recrystallization by thermal annealing were alternated until a final thickness of $\approx 300$ microns was obtained. The details of encapsulation and the homogenizing/ recrystallizing treatment are discussed below.

\subsection{Encapsulation}

Prior to any homogenization or annealing treatment, alloy pieces were always encapsulated in quartz tubes, using a dedicated encapsulation set-up. We started with $4 \mathrm{ft}$ long quartz tubing with an inner diameter of either $10.5 \mathrm{~mm}$ or $17 \mathrm{~mm}$. A hydrogen torch was used to divide the tube 
into two halves, each half having one closed end. The sample to be encapsulated was then inserted into one of the halves and allowed to rest against the closed end. The torch was then used to form a 'neck', i.e., a region with a narrowed cross section, on the tube. Since the tube is later sealed off at the location of this neck, the distance of the neck from the closed end of the tubing is the size of our final encapsulated tube.

The open end of the tubing was then connected to a roughing pump and pumped down to $10^{-2}$ torr. The tubing was then back-filled with Argon upto a partial pressure of 10 inches $\mathrm{Hg}$; i.e., until the indicator in the sub-atmospheric pressure gauge indicated 20 inches $\mathrm{Hg}$. This evacuation and backfilling was repeated two more times and finally the neck was sealed off with the torch, which allowed us to detach the sealed tube.

\subsection{Homogenization}

Homogenization/ recrystallization was conducted at $925^{\circ} \mathrm{C}$ for 8 hours in a tube furnace. The samples were then water quenched to retain the disordered FCC phase. This material consisting of the chemically disordered FCC phase shall be henceforth referred to as the base material.

\subsection{Annealing treatments}

$15 \mathrm{~mm} \times 5-8 \mathrm{~mm}$ samples were cut from the FCC base material using a cut-off saw and used for various annealing treatments, a synopsis of which is presented here. Samples of all four compositions were annealed at $600{ }^{\circ} \mathrm{C}$ and $700{ }^{\circ} \mathrm{C}$, i.e., below the eutectoid temperature $\left(730{ }^{\circ} \mathrm{C}\right)$, for different time durations, ranging from 15 minutes to 30 days. Samples with compositions within the range of the eutectoid isotherm were additionally continuously cooled from $750{ }^{\circ} \mathrm{C}$, in a programmable furnace. The final quench temperatures ranged from 600 to $690{ }^{\circ} \mathrm{C}$, hold times at the final temperature before quenching varied from 0 to 2 weeks. We also employed two different cooling rates: $20^{\circ} \mathrm{C} /$ day and $40^{\circ} \mathrm{C} /$ day. 


\subsection{X-Ray Diffraction (XRD)}

$\mathrm{XRD}$ investigation using $\mathrm{Cu}-\mathrm{K} \alpha$ radiation was conducted on the base material as well as on each $15 \mathrm{~mm}$ X 5-8 mm coupon upon completion of the annealing treatments. The samples were typically $\approx 400$ microns thick which was greater than the extinction length for $\mathrm{Cu} \mathrm{K \alpha} \mathrm{X}$-rays at all incident angles. The X'Pert Panalytical Pro Multi Purpose diffractometer was employed for this investigation. The XRD patterns indicate the phases present in the heat treated samples. A program called Magic Plot was used to fit the XRD peaks and a comparison of the peak intensities of the fundamental $\{002\}$ and superlattice $\{001\}$ reflections in the XRD pattern from a sample enabled estimation of the corresponding order parameter. Additional details regarding optimization of this technique for our sample compositions geometries have been included in Appendix 2.

\subsection{Vibrating Sample Magnetometry (VSM)}

VSM is a standard technique used to determine the magnetic properties of materials. VSM operates on the principle that the oscillatory motion of a magnetized sample resulting in changing magnetic flux will induce a voltage in a pickup coil, in accordance with Faraday's law of induction. The VSM set-up is illustrated schematically in Figure 2.2. Herein, we see that the sample is attached to the end of a long rod which is vibrated vertically with a certain frequency and amplitude. The rod is positioned between the pole pieces of an electromagnet so that a static magnetic field may magnetize the oscillating sample; and a set of pick up coils are mounted on these electromagnets. ${ }^{41,42}$ 


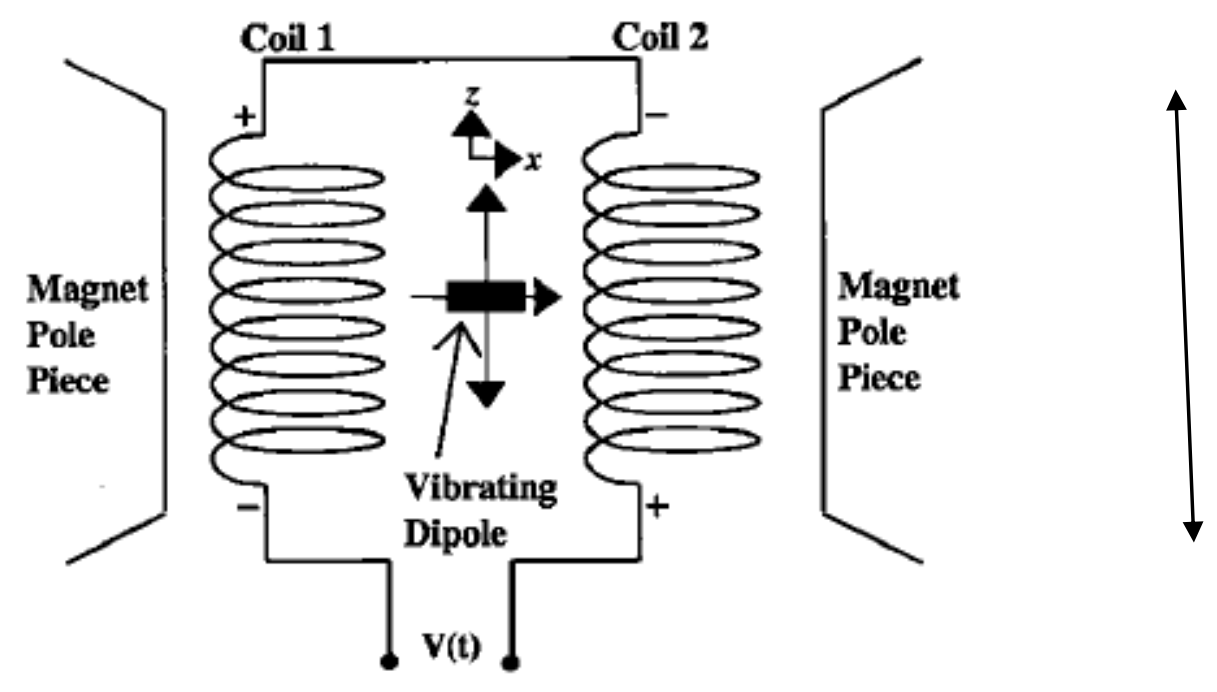

Figure 2.2: Schematic representation of the VSM set-up. ${ }^{43}$

The voltage induced in the pickup coil, $\mathrm{V}_{\text {coil }}$, is given by the following equation:

$\mathrm{V}_{\text {coil }}=\mathrm{d}(\Phi) / \mathrm{dt}=\mathrm{d}(\Phi) / \mathrm{dz} * \mathrm{~d}(\mathrm{z}) / \mathrm{dt}$

' $\Phi$ ' being the magnetic flux enclosed by the detector coil, ' $z$ ' the vertical position of the sample, and ' $t$ ' the time. For a sample oscillating sinusoidally, the voltage induced in the pickup coil is given as :

$\mathrm{V}_{\text {coil }}=2 \pi \mathrm{fN} \mu \mathrm{D} \sin (2 \pi v \mathrm{t})$

where $\mathrm{N}$ is a coupling constant, $\mu$ is the magnetic moment of the sample, $\mathrm{D}$ is the amplitude of oscillation, and $v$ is the frequency of oscillation. ${ }^{42}$ The induced voltage is thus proportional to the sample's magnetization. When a new static magnetic field is applied via the electromagnets, it produces a corresponding moment in the sample and a change in the induced voltage. Thus, the entire hysteresis curve may be generated by varying the static field applied to the vibrating sample.

This technique was employed to study the room temperature magnetic response of the alloys, using the Lakeshore 7407 magnetometer. The base material as well as annealed samples were 
first ground down to a thickness of $\approx 150$ microns using a succession of 180 grit, 600 grit, 800 grit and finally 1200 grit $\mathrm{SiC}$ abrasive discs. Subsequently, discs $3 \mathrm{~mm}$ in diameter were punched out using a Gatan Disc Punch.

The samples were first degaussed to eliminate residual magnetization for proper determination of the initial magnetization curve. Then, a high number of data points were collected to generate the entire M-H hysteresis loop. The magnetic field was applied in the plane of the sample. The sample shape helped minimize the demagnetizing field and its effect on the MH loops.

\subsection{Curie temperature determination}

We determined the Curie temperatures of predominantly-L1 $\mathrm{L}_{0} \mathrm{Co}_{41.7} \mathrm{Pt}_{58.3}$ and $\mathrm{L1}_{2} \mathrm{Co}_{37.6} \mathrm{Pt}_{62.4}$ samples. Disc specimens previously used for VSM were attached to ferrite or alnico magnets and gradually heated in a tube furnace. The temperature at which the disc was detached from the magnets was recorded as the Curie temperature for the corresponding composition.

\subsection{Transmission Electron Microscopy (TEM)}

Samples displaying interesting magnetic properties were selected for conventional TEM investigation. TEM samples were prepared as follows. Annealed samples were ground down to $\approx 150$ microns using 180 grit and 600 grit $\mathrm{SiC}$ abrasive discs and $3 \mathrm{~mm}$ diameter discs were punched out using a Gatan Disc Punch. The disc samples were further ground to a thickness of 50-80 microns using a succession of 600 grit, 800 grit and 1200 grit $\mathrm{SiC}$ abrasive discs, to ensure a smoother surface finish. It is recommended that the thickness of the disc be closer to 50 microns than 80 microns. This will ensure quicker dimpling without compromising the ease of handling of the foil specimens. Grinding was followed by dimpling, i.e., selectively thinning the center of the disc specimens to 10-20 microns using a dimpling grinder. A 3-micron diamond paste was used for dimpling with a grinding rate of 2 microns/minute under a grinding wheel load of $40 \mathrm{~g}$, for the bulk of the thinning. In the final stages of dimpling, a 1-micron diamond paste was employed, with a grinding rate of $1 \mathrm{micron} / \mathrm{minute}$ minute under a grinding wheel load of $15 \mathrm{~g}$, to attain a good surface polish. During all stages, the polishing wheel and sample stage were gently rotated. Some of our earliest samples, however, were not dimpled before ion- 
milling. Additional details regarding the grinding/polishing and dimpling techniques using available apparatus are included in Appendix 3.

The dimpled samples were finally ion-milled to electron transparency, at low temperatures (-100 to $-50{ }^{\circ} \mathrm{C}$ ). High angle milling produced artifacts in the TEM sample, as shown in Figure 2.3. Thus, it is of utmost importance to use a very shallow incident ion angle during milling to ensure high sample quality and minimize/avoid artifacts. To produce high quality samples, we milled the sample from both sides with the top gun releasing ions at an angle of 5 degrees and the bottom gun firing ions at an angle of 3 degrees to the surface of the sample, with an incident ion energy of $5 \mathrm{keV}$, until perforation was achieved. Following perforation, the sample was milled for 30 minutes with the top gun releasing ions at an angle of 5 degrees and the bottom gun firing ions at a reduced angle of 2 degrees to the surface of the sample, with a lower incident ion energy, $3 \mathrm{keV}$. This allowed for a larger electron- transparent area. The sample surface was then cleaned up by gently milling with an incident ion energy of $2.5 \mathrm{keV}$ for 5 minutes The samples thus prepared were carefully cleaned with ethanol and then analyzed using the JEOL $2000 \mathrm{FX}$, using both bright field and dark field imaging techniques.

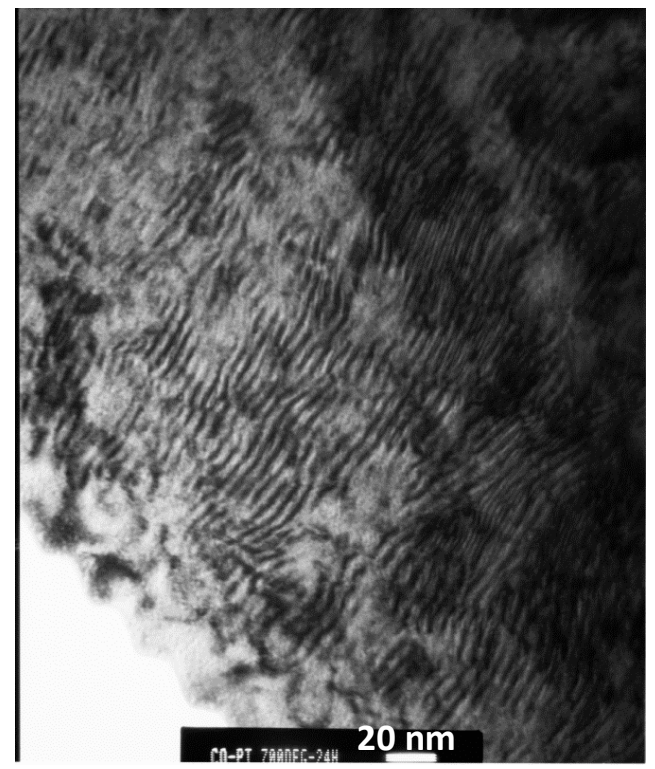

Figure 2.3: $\mathrm{BF}$ image from an early TEM sample evidencing ion milling damage as a consequence of high angle milling using top and bottom milling angles of $10^{\circ}$. Such damage was prevented in subsequent samples by using shallow milling angles. 


\subsection{Differential Scanning Calorimetry (DSC)}

Thermal characterization using DSC was conducted at Northeastern University in the Laura Lewis group to identify the solid-solid eutectoid transition from caloric changes detected during a controlled temperature program. A Netzsch STA 449 F3 Differential Scanning Calorimeter was employed to perform heating/cooling experiments at heating/cooling rates of $10 \mathrm{~K} / \mathrm{min}$. The experiments were performed in an argon atmsphere to prevent oxidation of the samples. The sample slow cooled from 750 to $650{ }^{\circ} \mathrm{C}$ and then water quenched was used for the determination of the eutectoid temperature. 


\section{Compositions bordering the eutectoid isotherm: $\mathrm{Co}_{41.7} \mathrm{Pt}_{58.3}$ and $\mathrm{Co}_{37.6} \mathbf{P t}_{62.4}$}

This detailed study of compositions marginally outside the eutectoid reaction isotherm provides an understanding of the behavior of the $\mathrm{L}_{0}$ and $\mathrm{L}_{2}$ phases for compositions in the vicinity of the two phase region, i.e., well off the nominal stoichiometries of $\mathrm{CoPt}$ and $\mathrm{CoPt}_{3}$. It also facilitates a comparison of the microstructure and properties between these alloys versus alloys located on the eutectoid isotherm that exhibit the nanochessboard structure.

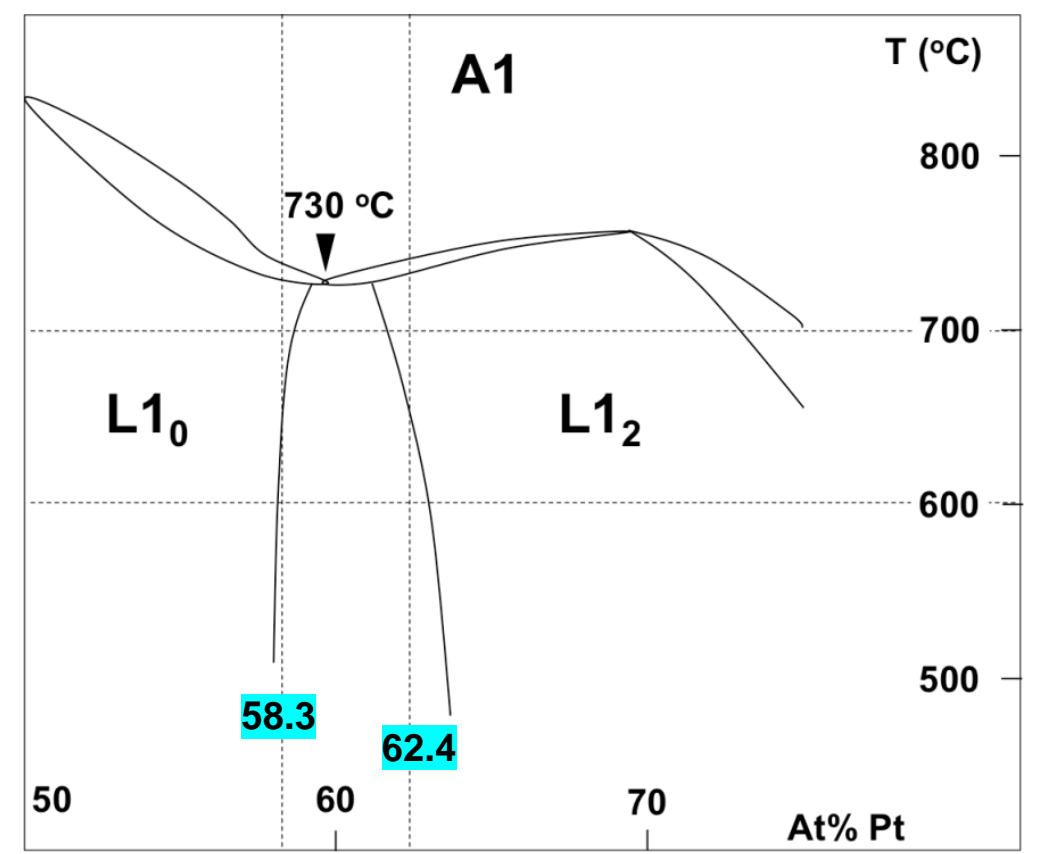

Figure 3.1: Phase diagram near the eutectoid, as determined by Leroux, et al. ${ }^{1}$. The vertical dashed lines show the compositions under consideration in this chapter. The horizontal lines indicate the isothermal aging temperatures that were studied in detail.

$\mathrm{Co}_{41.7} \mathrm{Pt}_{58.3}$ was the first composition to be processed and investigated during the course of this research. As illustrated in Figure 3.1, this composition has a slightly lower Pt content with respect to the compositions on the eutectoid reaction isotherm, i.e., it lies in the $\mathrm{L} 1_{0}$ regime at the published eutectoid temperature. Following appropriate annealing treatment, this alloy exhibited the highest coercivity and the maximum exchange coupling among all compositions investigated, associated with formation of $\mathrm{L1}_{0}$ nanoparticles. The other composition studied, $\mathrm{Co}_{37.6} \mathrm{Pt}_{62.4}$ is also 
indicated on the phase diagram in Figure 3.1, and has a Pt content slightly higher than the compositions on the eutectoid reaction isotherm, i.e., it lies in the $\mathrm{L}_{2}$ regime at the eutectoid temperature. Upon annealing, this alloy ordered to $\mathrm{L}_{2}$ and consequently evidenced soft magnetic behavior. The dependence of magnetic properties, phases present and microstructure on annealing time and temperature for both these alloys is presented and discussed in this chapter.

\section{$\underline{\text { A.Results: }}$}

\subsection{Magnetic Properties}

\subsubsection{Curie Temperature}

Our experiments reveal that the $\mathrm{Co}_{41.7} \mathrm{Pt}_{58.3}$ alloy contained predominantly the $\mathrm{L}_{0}$ phase upon annealing for a prolonged duration. This phase evidenced the maximum degree of order possible for the alloy composition and demonstrated a Curie temperature of $419^{\circ} \mathrm{C}$. On the other hand, the $\mathrm{Co}_{37.6} \mathrm{Pt}_{62.4}$ alloy transformed to the $\mathrm{L1}_{2}$ phase, also showing the highest extent of ordering theoretically ascertained for this composition, in response to extended annealing. The $\mathrm{L1}_{2}$ phase had a Curie temperature of $354{ }^{\circ} \mathrm{C}$. From the literature, we know that stoichiometric $\mathrm{L} 1_{0}-$ structured CoPt has a Curie Temperature of $567{ }^{\circ} \mathrm{C}{ }^{9}$ whereas stoichiometric $\mathrm{L1}_{2}$ - structured $\mathrm{CoPt}_{3}$ has a Curie temperature of $15{ }^{\circ} \mathrm{C}{ }^{5}$. So, while stoichiometric $\mathrm{L}_{2}$ would have been expected to be paramagnetic at room temperature, the non-stoichiometric phase is ferromagnetic. Thus, we see that deviations from stoichiometry significantly affect the value of the Curie temperature and magnetic behavior of the alloy. The Curie temperatures of the two compositions bracketing the two phase region would also apply to the compositions within the two-phase coexistence region, and hence, to the chessboard structure. The variation of the Curie temperature with composition is graphically depicted in Figure 3.2. 


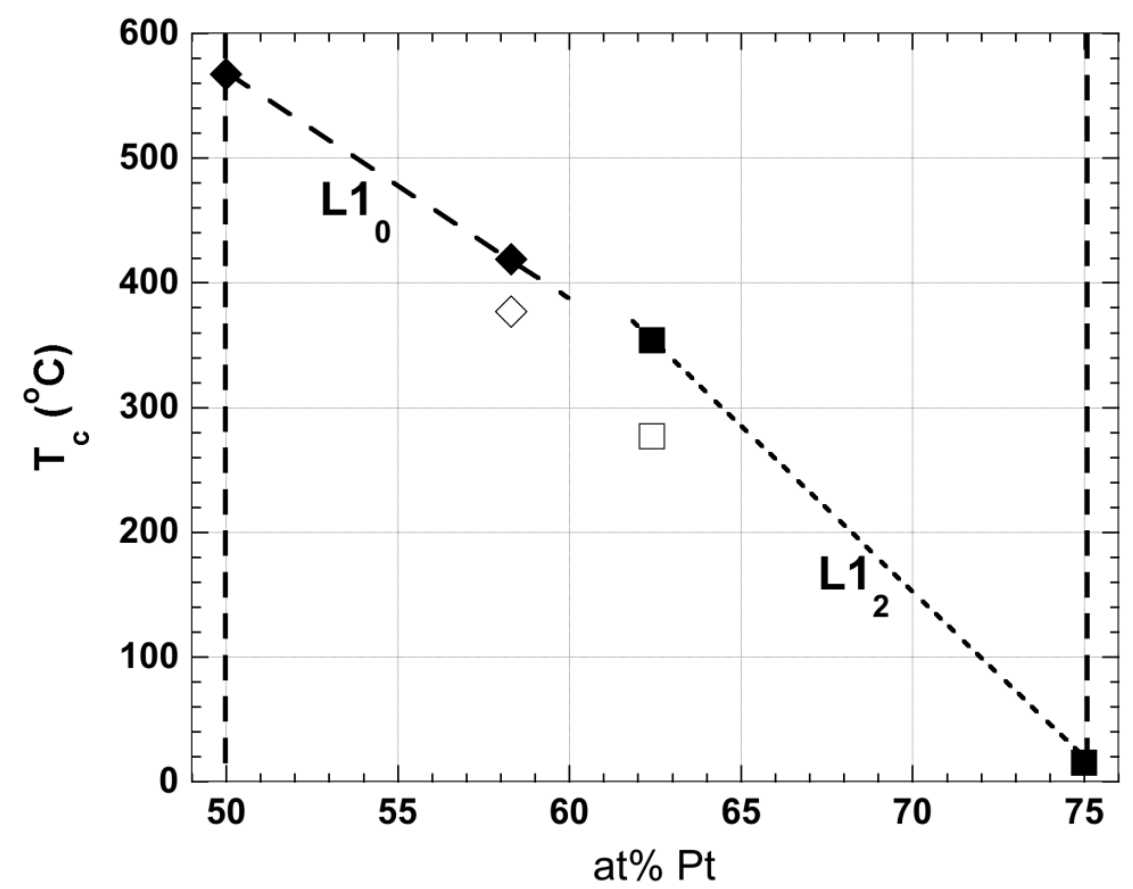

Figure 3.2: Curie temperatures for the two most highly transformed samples. $\mathrm{Co}_{41.7} \mathrm{Pt}_{58.3}$ annealed at $700{ }^{\circ} \mathrm{C}$ for 504 hours, containing predominantly $\mathrm{L} 1_{0}$ is indicated by the filled diamond. $\mathrm{Co}_{37.6} \mathrm{Pt}_{62.4}$ annealed at $700{ }^{\circ} \mathrm{C}$ for 233 hours contains $\mathrm{L1}_{2}$, and is indicated by the filled square. Also shown are Curie temperature values for the stoichiometric $\mathrm{CoPt}^{9}$ and $\mathrm{CoPt}_{3}$ phases 5 , indicated by the filled symbols, as well as for our compositions, extrapolated from data by Mehaddene, et al. ${ }^{5}$, denoted by open symbols. Lines are just intended as guides to the eye.

Magnetic properties, viz. the coercivity, saturation magnetization, remanence, remanence ratio and the maximum energy product, are observed to change significantly with annealing temperature and time, corresponding to a related change in microstructural length scale, phases present, pinning mechanisms etc. We have extensively studied the variation of magnetic properties with annealing time and temperature and present the results below. 


\section{$\underline{3.1 .2 \mathrm{Co}_{41.7}} \underline{\mathrm{Pt}_{58.3}}$}

Samples cut from the base material were isothermally annealed at $600{ }^{\circ} \mathrm{C}$ for $16-720$ hours, or at $700{ }^{\circ} \mathrm{C}$ for 15 minutes -504 hours. The soft ferromagnetic base material underwent magnetic hardening, the degree of which depended on the aging time and temperature. The variation of magnetic properties with aging time and temperature is shown in Figure 3.3.

Of all samples annealed at $600{ }^{\circ} \mathrm{C}$ in the course of this research work, an annealing time of 96 hours produced peak hardness corresponding to a coercivity $\left(H_{c}\right)$ of $\approx 4000$ Oe. The coercivity was observed to drop to a local minimum upon further aging, to a total of 168 hours. Continued annealing resulted in a gradual rise in coercivity. $\mathrm{H}_{\mathrm{c}}$ returned to $\approx 4000$ Oe upon annealing for 720 hours at $600{ }^{\circ} \mathrm{C}$. A sample annealed at $575{ }^{\circ} \mathrm{C}$ for 96 hour produced an even higher coercivity of 4600 Oe.

Annealing at $700{ }^{\circ} \mathrm{C}$ produced a more rapid evolution of magnetic properties due to accelerated kinetics at this higher temperature, and more complex age hardening behavior, as indicated by a comparison of the shapes of the age hardening curves. The coercivity attained an observed maximum value of 2300 Oe in only 2.5 hours. Presumably, the actual maximum occurs at an annealing time intermediate between 0.5 and 2.5 hours. With subsequent annealing, the value of $\mathrm{H}_{\mathrm{c}}$ initially fell, later rising to a secondary local maximum value of 1000 Oe after 24 hours of aging. Upon further aging, this value decreased to yet another secondary minimum at 120 hours, followed thereafter by a gradual increase upon extended aging to the maximum annealing time examined, 504 hours. A sample step cooled from $740{ }^{\circ} \mathrm{C}$ to $700{ }^{\circ} \mathrm{C}$ at the rate of $10{ }^{\circ} \mathrm{C}$ per 12 hours and then held at $700{ }^{\circ} \mathrm{C}$ for 72 hours, i.e., heat treated for a total of 120 hours, shows a significantly higher coercivity, $740 \mathrm{Oe}$, versus the 270 Oe corresponding to isothermal annealing at $700{ }^{\circ} \mathrm{C}$. The magnetic age hardening behavior is shown in Fig 3.3a. The time and temperature dependence of the remanent magnetization $\left(\mathrm{M}_{\mathrm{r}}\right)$, shown in Figure 3.3b, qualitatively showed a trend similar to the coercivity. 

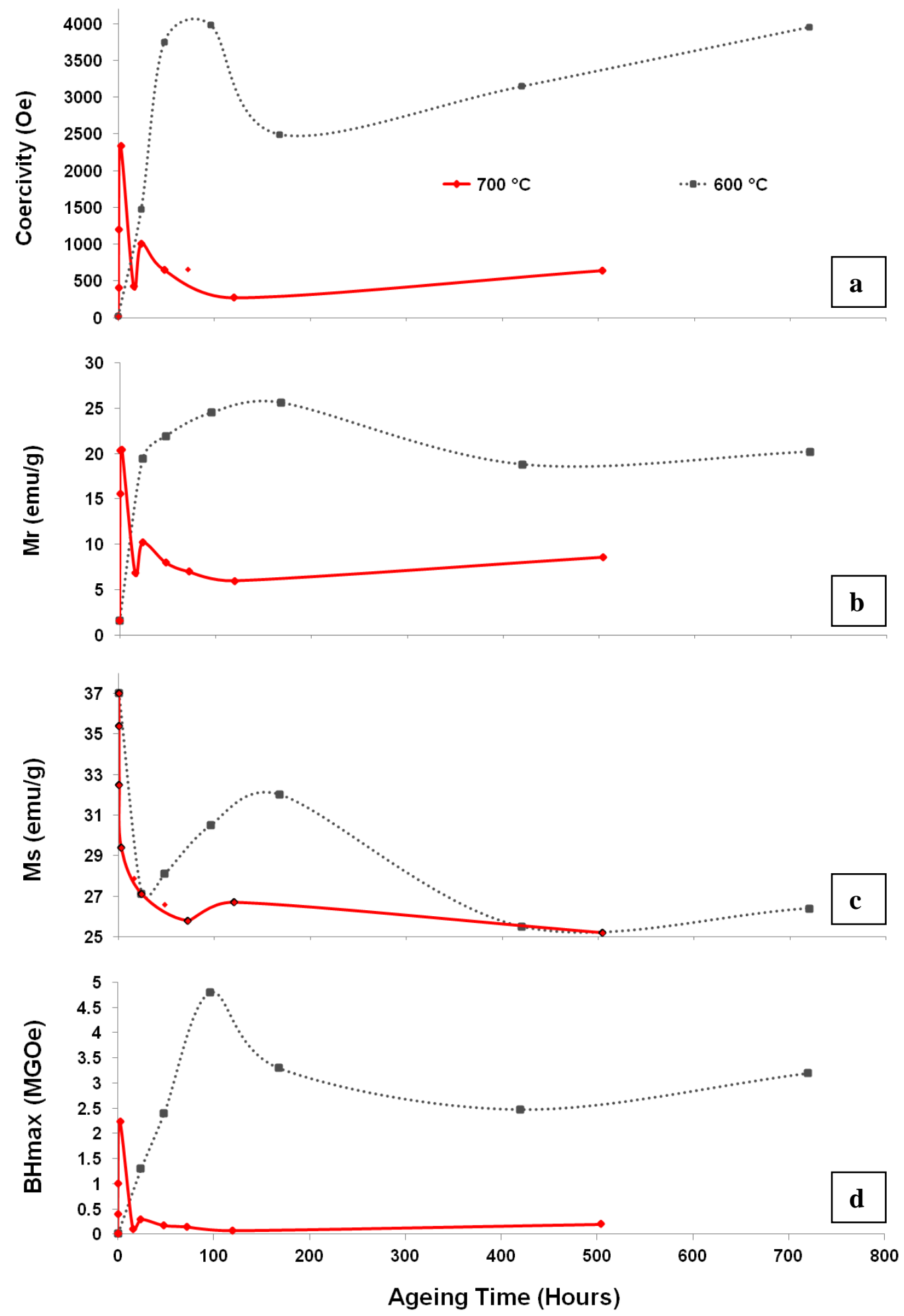
Figure 3.3: Variation of magnetic properties (a) Coercivity, (b) remanence and (c) saturation magnetization and (d) BHmax, as a function of aging time and temperature. Red circles indicate an annealing temperature of $700{ }^{\circ} \mathrm{C}$ while gray squares denote $600{ }^{\circ} \mathrm{C}$. Lines through the data are intended only as guides to the eye.

The annealing-time and temperature dependence of the saturation magnetization, $\mathbf{M}_{\mathrm{s}}$ is shown in Figure 3.3c. $\mathrm{Co}_{41.7} \mathrm{Pt}_{58.3}$ exhibited the highest $\mathrm{M}_{\mathrm{s}}(37 \mathrm{emu} / \mathrm{g})$ for the disordered A1 (FCC) phase; assuming lower values upon undergoing the disorder $\rightarrow$ order phase transformation in response to annealing treatments. Interestingly, a significant secondary maximum in $\mathbf{M}_{\mathrm{s}}$ was observed, corresponding to an aging time of 168 hours at $600{ }^{\circ} \mathrm{C}$, which may be potentially attributed to the presence of an intermediate metastable phases such as the L1', which disappear upon further annealing.

Attainment of a steady value of the saturation magnetization may serve to indicate the completion of the ordering transformation. From Figure 3.3c, we see that samples annealed at $700{ }^{\circ} \mathrm{C}$ for an extended duration of 504 hours appears to have attained or almost attained a steady state value of $\mathrm{M}_{\mathrm{s}}, 26 \mathrm{emu} / \mathrm{g}$. Our XRD results below will further confirm the completion of ordering transformation at this stage. XRD also indicates that annealing at $600{ }^{\circ} \mathrm{C}$ did not lead to complete ordering, even after 720 hours, owing to relatively sluggish kinetics at that temperature.

The $\mathrm{BH}_{\max }$ showed a trend similar to the coercivity and the saturation magnetization (Figure $3.3 \mathrm{~d})$. In the process of determining the impact of annealing conditions on magnetic properties, we observed that samples annealed at $600{ }^{\circ} \mathrm{C}$ for 96 hours showed the best permanent magnet magnetic properties, of all samples annealed at $600{ }^{\circ} \mathrm{C}$ and $700{ }^{\circ} \mathrm{C}$. In addition to maximum coercivity, this annealing treatment resulted in maximum exchange coupling between the constituent phases, as determined by the corresponding remanence to saturation ratio $\left(\mathrm{M}_{\mathrm{r}} / \mathrm{M}_{\mathrm{s}}\right)$ of 0.8 or $80 \%$. Although exhibiting a higher coercivity, the sample annealed for the same duration, i.e., 96 hours at $575{ }^{\circ} \mathrm{C}$ had a slightly lower value of $\mathrm{M}_{\mathrm{r}} / \mathrm{M}_{\mathrm{s}}, 0.77$. The variation of $\mathrm{M}_{\mathrm{r}} / \mathrm{M}_{\mathrm{s}}$ with aging conditions and duration is shown in Figure3.4. 


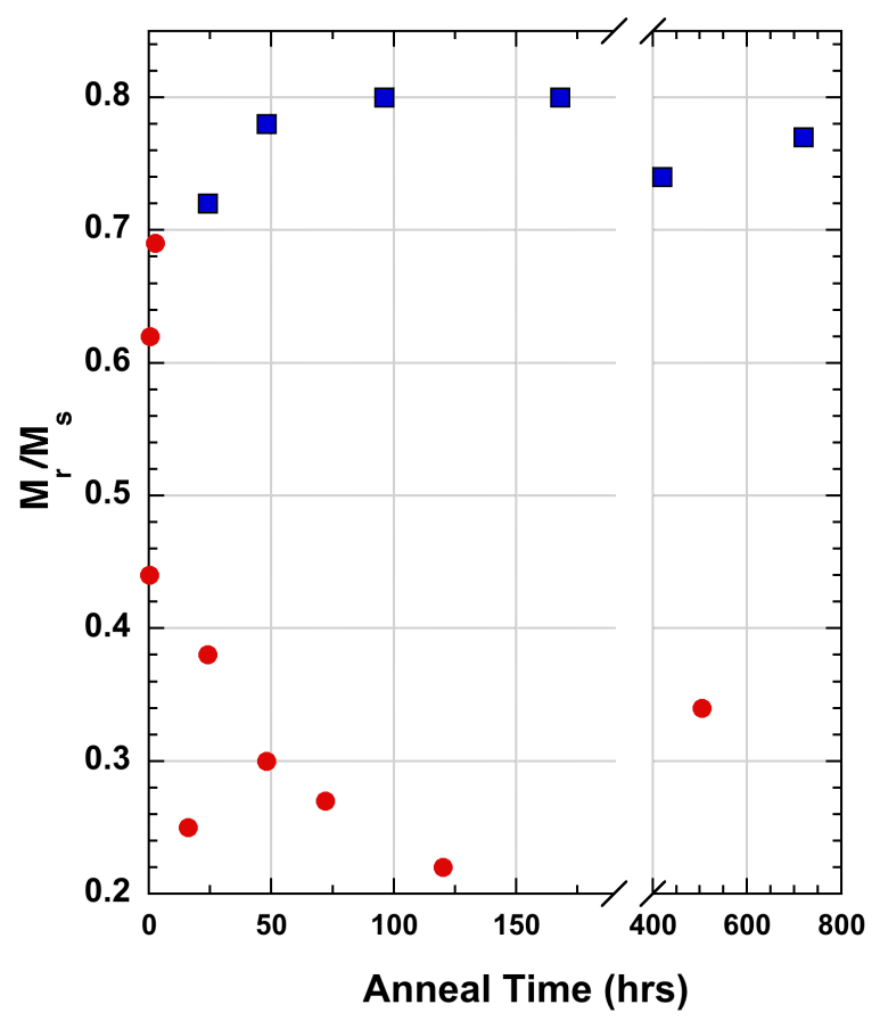

Figure 3.4: Remanence ratio vs. aging time at $600{ }^{\circ} \mathrm{C}$ (blue squares) and $700{ }^{\circ} \mathrm{C}$ (red circles).

The M-H hysteresis loops of the base material and peak hardness samples obtained at $600{ }^{\circ} \mathrm{C}$ and $700{ }^{\circ} \mathrm{C}$ are shown in Figure 3.5(a). The initial magnetization curves reflect the magnetization reversal mechanisms; and have been highlighted in Figure 3.5(b). The initial magnetization curve for the base material demonstrated an extremely high susceptibility, $\mathrm{M} / \mathrm{H}$, in agreement with a soft, nucleation-type behavior. On the other hand, the peak hardness sample annealed that $600{ }^{\circ} \mathrm{C}$ for 96 hours revealed an especially gradual change in magnetization in response to applied magnetic field. The initial magnetization behavior was indicative of domain wall pinning, as will be further justified in the discussion section. The high coercivity sample annealed at $700{ }^{\circ} \mathrm{C}$ for 2.5 hours exhibited a low initial susceptibility, followed later by a sharp rise. This suggests that the domain wall pinning mechanism operational at low applied fields was later replaced by another mechanism at higher applied field, following the discontinuity in the initial magnetization curve. A weak pinning mechanism would effectively explain the observed 
scenario at higher applied fields, with the susceptibility lying intermediate between the typical values for nucleation and domain wall pinning in that regime.
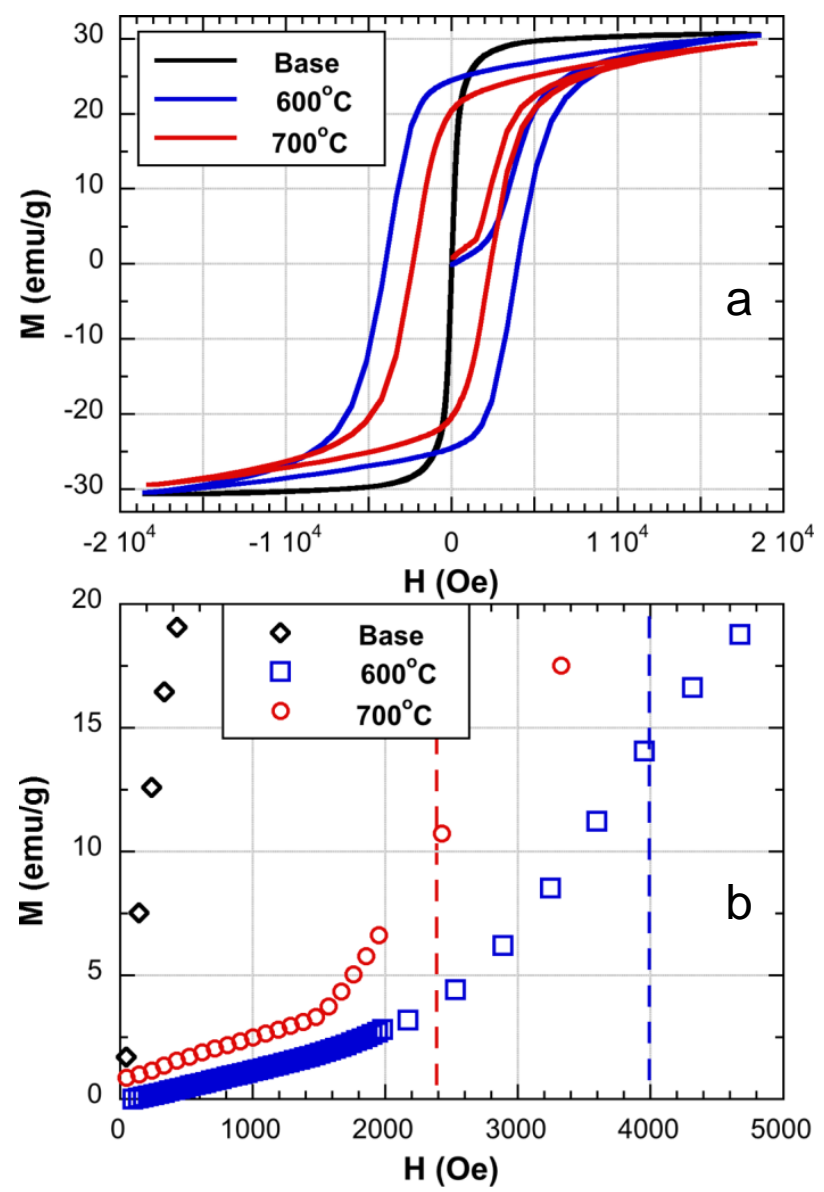

Figure 3.5: (a) A comparison of the $\mathrm{M}-\mathrm{H}$ hysteresis loops comparing the base material with the peak hardness samples annealed at $600{ }^{\circ} \mathrm{C}$ for 96 hours and $700{ }^{\circ} \mathrm{C}$ for 2.5 hours, for the $\mathrm{Co}_{41.7} \mathrm{Pt}_{58.3}$ alloy. (b) An enlarged view highlighting the initial magnetization behavior for these samples. The dashed lines indicate the corresponding coercivities.

\subsection{3. $\mathrm{Co}_{37.6} \underline{\mathrm{Pt}}_{62.4}$}

The second alloy processed was the more Pt-rich $\mathrm{Co}_{37.6} \mathrm{Pt}_{62.4}$. Samples were annealed by first stepwise cooling from $740{ }^{\circ} \mathrm{C}$ to $700{ }^{\circ} \mathrm{C}$ at the rate of $10^{\circ} \mathrm{C}$ per 12 hours and then holding at 700 ${ }^{\circ} \mathrm{C}$ for 24,34 and 223 hours. Other samples from were directly annealed at $600{ }^{\circ} \mathrm{C}-$ for 96 hours, 
to facilitate comparison with the $\mathrm{Co}_{41.7} \mathrm{Pt}_{58.3}$ alloy subjected to an identical aging treatment; as well as for an extended time of 310 hours.

The alloy continued to display soft magnetic properties, similar to those of the single phase FCC base material, even after prolonged annealing. Compared to $\mathrm{Co}_{41.7} \mathrm{Pt}_{58.3}$, the reduced Co content in this $\mathrm{Co}_{37.6} \mathrm{Pt}_{62.4}$ alloy produced a relatively smaller $\mathrm{M}_{\mathrm{s}}$ in the FCC-base material, $32 \mathrm{emu} / \mathrm{g}$, which decreased to $\approx 30 \mathrm{emu} / \mathrm{g}$ after aging for long durations. The remanence-to-saturation ratios were found to be particularly low, $\approx 0.1$, in the samples annealed at $700{ }^{\circ} \mathrm{C}$; though comparatively higher, $\approx 0.3$, in samples annealed at $600{ }^{\circ} \mathrm{C}$, likely stemming from the finer microstructural length-scales at the lower temperature. Samples isothermally annealed at $600{ }^{\circ} \mathrm{C}$ also resulted in relatively harder magnetic properties. This possibly arises from the fact that a higher density of anti-phase boundaries (APBs), which weakly pin domain walls, was available at $600{ }^{\circ} \mathrm{C}$, resulting from increased nucleation density and decreased recovery at that temperature, compared to $700{ }^{\circ} \mathrm{C}$. $\mathrm{BH}_{\max }$ was observed to be very low, $\approx 0.005 \mathrm{MG}$ Oe. A summary of the magnetic properties as a function of annealing time and temperature is presented in Table 1.

\begin{tabular}{|c|c|c|c|}
\hline $\begin{array}{c}\text { Annealing } \\
\text { Time } \\
\text { (Hours) }\end{array}$ & $\begin{array}{c}\mathbf{M}_{\mathbf{s}} \\
\text { (emu/g) }\end{array}$ & $\begin{array}{c}\mathbf{M}_{\mathbf{r}} \\
\text { (emu/g) }\end{array}$ & $\mathbf{H}_{\text {ci }}$ (Oe) \\
\hline \multicolumn{4}{|c|}{ Base } \\
\hline 0 & 32.1 & 1.9 & 19 \\
\hline Slow cooling from $740{ }^{\circ}$ C to $700{ }^{\circ} \mathrm{C}$, annealing \\
at $700{ }^{\circ} \mathrm{C}$ \\
\hline 24 & 33 & 3 & 41 \\
34 & 29.3 & 1.9 & 41 \\
223 & 30.6 & 1.6 & 39 \\
\hline \multicolumn{4}{|c|}{ Annealing at $600{ }^{\circ} \mathrm{C}$} \\
\hline 96 & 31.9 & 9.9 & 158 \\
310 & 30.4 & 9.8 & 175 \\
\hline
\end{tabular}

Table 3.1: Variation of magnetic properties viz. saturation magnetization, remanent magnetization and coercivity, with annealing in $\mathrm{Co}_{37.6} \mathrm{Pt}_{62.4}$. 


\subsection{Phase Identification and Order Parameter Determination}

\subsection{1. $\mathrm{Co}_{41.7} \underline{\mathrm{Pt}_{58.3}}$}

X-Ray Diffraction (XRD) patterns from representative samples are shown in Figure3.6. From these plots, we learn that the FCC base material underwent the disorder $\rightarrow$ order transformation with annealing. Chemical ordering, to either the $\mathrm{L}_{0}$ or $\mathrm{L}_{2}$ phase, was indicated by the appearance of the (001), (110) and higher-index superlattice reflections, which are not allowed for the disordered FCC phase ${ }^{44}$. As the $\mathrm{Co}_{41.7} \mathrm{Pt}_{58.3}$ alloy transformed to $\mathrm{L}_{0}$ upon annealing, a peak assigned as (002) emerged and grew from the $\{200\}$ peak. This splitting of the $\{200\}$ peak into $\{200\}$ and (002) peaks arises from tetragonality of the ordered phase and is thus the signature of the $\mathrm{L}_{0}$ phase; it cannot occur in the $\mathrm{L}_{2}$ phase. Together with the observed hard magnetic properties, this information from XRD underscores the fact that the A1 phase transformed primarily to $\mathrm{L}_{0}$ for the case of $\mathrm{Co}_{41.7} \mathrm{Pt}_{58.3}$. The evolving (002) peak position may indicate a continuous ordering transition, although it could also arise from evolving coherency strain, depending on the relative volume fractions and lengthscales of the constituent phases. A fixed peak position accompanied only by a change in peak intensity would have been expected for a nucleation and growth type transition.

It may be pointed out that the tetragonal distortion indicated by the $\{200\} /(002)$ peak splitting was not evident in case of the peak hardness sample at either annealing temperatures. Small quantities of the $\mathrm{L}_{2}$ were also inferred to form after annealing at elevated temperatures for extended durations. Our analysis was based on a series of observations, including the timedependence of magnetic properties, the relative intensities of the $\{200\}$ and (002) peaks, order parameters as well as the 'splitting' of the (001) peak, modeled by a 2-peak fit of this wide superlattice reflection, which occurred after prolonged annealing. While the (001) peak is a permitted reflection for the $\mathrm{L} 1_{0}$ phase, its splitting due to tetragonal distortion is forbidden, based on structure factor calculations. Thus, the 'splitting' of the (001) peak arises from the emergence of the $\mathrm{L}_{2}$ phase; and actually represents the presence of both ordered phases located at slightly different values of $2 \theta$. 


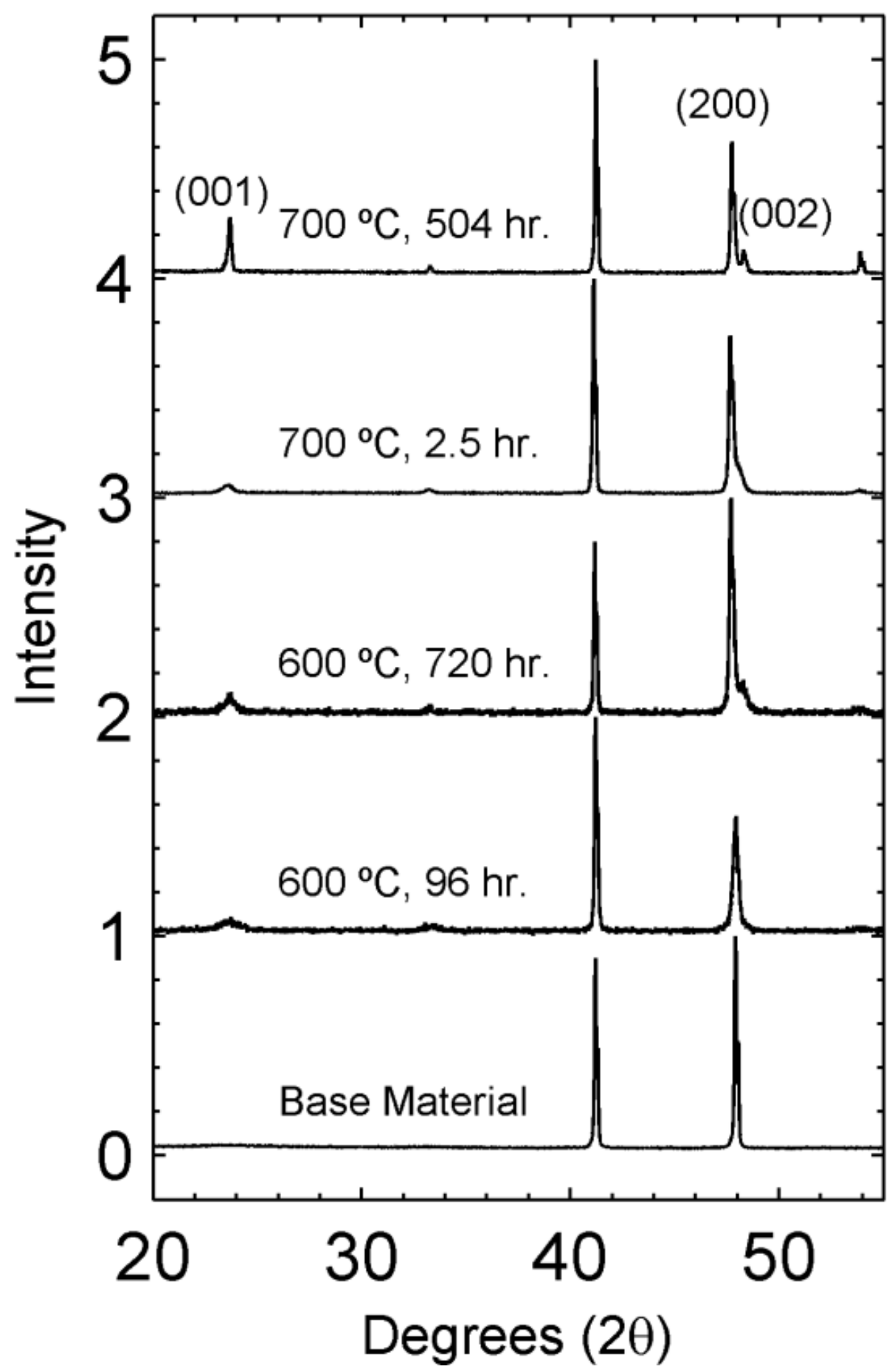

Figure 3.6: XRD spectra from the $\mathrm{Co}_{41.7} \mathrm{Pt}_{58.3}$ alloy for (a) base material, and samples annealed at (b) $600{ }^{\circ} \mathrm{C}$ for 96 hours, (c) $600{ }^{\circ} \mathrm{C}$ for 720 hours, (d) $700{ }^{\circ} \mathrm{C}$ for 2.5 hours, and (e) $700{ }^{\circ} \mathrm{C}$ for 504 hours. 
A comparison of the (001) and $\{200\} /(002)$ reflections in the samples annealed at $600{ }^{\circ} \mathrm{C}$ for 720 hours and at $700{ }^{\circ} \mathrm{C}$ for 504 hours is presented in Figure 3.7. Due to the potential presence of three or more phases (untransformed $\mathrm{FCC}, \mathrm{L1}_{0}, \mathrm{L1}_{2}$, potentially $\mathrm{L} 1^{\prime}$ ) in the sample annealed at $600{ }^{\circ} \mathrm{C}$ for 720 hours, an analysis of the data for lattice parameters, peak widths, order parameters, and particle sizes is particularly challenging. On the basis of the volume fraction calculations below, we conclude that samples annealed at $700{ }^{\circ} \mathrm{C}$ for an extended time of 504 hours were better behaved with data analysis producing internally consistent results.

The order parameters of the $\mathrm{L}_{0}$ phase were calculated for the extended anneals at both $600{ }^{\circ} \mathrm{C}$ and $700{ }^{\circ} \mathrm{C}$. This was accomplished by comparing the integrated intensities of the (001) superlattice and the (002) fundamental peaks, obtained from fitting analysis of the data in Figure 3.7. This eliminated the influence of texture on the relative peak intensities, and hence, on order parameter calculations. 

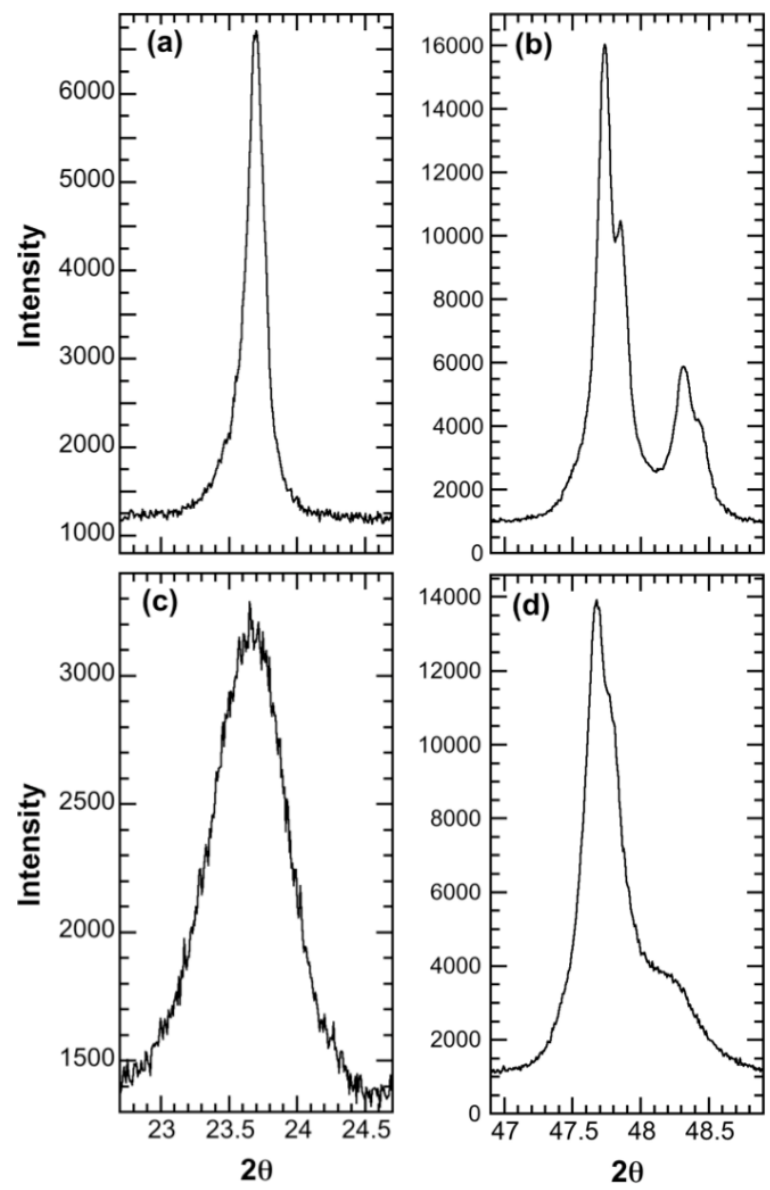

Figure 3.7: (a) (001) and (b) \{200\}/ (002) X-ray diffraction peaks from the annealing treatment at $700{ }^{\circ} \mathrm{C}$ for 504 hours. (c) (001) and (d) \{200\}/ (002) peaks from the annealing treatment at $600{ }^{\circ} \mathrm{C}$ for 720 hours. Based on appropriate peak fitting, the (001) peak actually reflected contributions from two components: $(001)_{\mathrm{L} 10}$ and $\{100\}_{\mathrm{L} 12}$, occurring at slightly different values of $2 \theta$.

The general procedure for order parameter calculation of a non-stoichiometric $\mathrm{L}_{0}$ phase is illustrated below:

$\mathbf{I}=\mathbf{F}^{2} \cdot \mathbf{m} \cdot \mathbf{L P}$

where $\mathbf{I}$ is the peak intensity; $\mathbf{F}$ is the structure factor and $\mathbf{m}$ is the peak multiplicity. $\mathbf{L P}$ is the Lorentz Polariation factor $=\left[1+\cos ^{2}(2 \theta)\right] /\left[\sin ^{2}(2 \theta) * \cos (\theta)\right]$, at the corresponding peak 
position, $2 \theta$. The subscripts $\mathbf{s}$ and $\mathbf{f}$ are used to denote superlattice and fundamental reflections, respectively.

$\mathbf{I}_{\mathrm{s}}=\mathbf{F}_{\mathrm{s}}{ }^{2} \cdot \mathbf{m}_{\mathrm{s}} \cdot \mathbf{L} \mathbf{P}_{\mathrm{s}} \quad \ldots(\mathrm{Eq} \cdot 3 \cdot 1 \mathrm{~A})$

Here, $\left.\mathrm{F}_{\mathrm{s}}=\eta .2\left(\mathrm{f}_{\mathrm{Pt}, \mathrm{s}}-\mathrm{f}_{\mathrm{Co}, \mathrm{s}}\right)\right]$, the ' $\eta$ ' representing the order parameter ${ }^{44}$;

For the (001) superlattice reflection occuring at $2 \theta_{001} \approx 23.7^{\circ}$,

$\mathrm{F}_{001}=\eta .2\left(\mathrm{f}_{\mathrm{Pt}, 001}-\mathrm{f}_{\mathrm{Co}, 001}\right)$

For $\left[\sin \left(\theta_{001}\right)\right] / \lambda=[\sin (23.7 / 2)] / 1.54 \AA, \mathrm{f}_{\mathrm{Pt}, 001}=71.84$ and $\mathrm{f}_{\mathrm{Co}, 001}=23.64$;

$\mathbf{F}_{001}=\eta * 2(71.84-23.64)$.

$\mathbf{m}_{001}=2$.

$\mathrm{LP}_{\mathbf{0 0 1}}=\left[1+\cos ^{2}\left(2 \theta_{001}\right)\right] /\left[\sin ^{2}\left(2 \theta_{001}\right) * \cos \left(\theta_{001}\right)\right] ;$ here $\mathbf{L} \mathbf{P}_{\mathbf{0 0 1}}=\mathbf{1 1 . 6 3}$

Thus, Eq. 3.1A may be re-written as $\mathbf{I}_{001}=[\eta * \mathbf{2}(\mathbf{7 1 . 8 4}-\mathbf{2 3 . 6 4})]^{2} * \mathbf{2} * \mathbf{1 1 . 6 3} \ldots$ (Eq. 3.2)

$\mathrm{I}_{\mathrm{f}}=\mathrm{F}_{\mathrm{f}}^{2} \cdot \mathrm{m}_{\mathrm{f}} \cdot \mathrm{LP}_{\mathrm{f}} \quad$ (E) $\left.\mathrm{Eq} \cdot 3 \cdot 1 \mathrm{~B}\right)$

For the (002) fundamental reflection occuring at $2 \theta=48.4^{\circ}$,

$\mathrm{F}_{\mathrm{f}}=4\left(\mathrm{X}_{\mathrm{Co}} \mathrm{f}_{\mathrm{Co}, 002}+\mathrm{X}_{\mathrm{Pt}} \mathrm{f}_{\mathrm{Pt}, 002}\right), \mathrm{X}$ being the mole fraction ${ }^{44}$. Here, $\mathrm{X}_{\mathrm{Co}} \approx 0.4, \mathrm{X}_{\mathrm{Co}} \approx 0.6$.

For $\left[\sin \left(\theta_{002}\right)\right] / \lambda=[\sin (48.4 / 2)] / 1.54 \AA$, $\mathrm{f}_{\mathrm{Pt}, 002}=61.6$ and $\mathrm{f}_{\mathrm{Co}, 002}=19.04$;

$\mathbf{F}_{002}=4[(0.4 * 19.04)+(0.6 * 61.6)]$

$\mathbf{m}_{002}=2$.

$\mathrm{LP}_{002}=\left[1+\cos ^{2}\left(2 \theta_{002}\right)\right] /\left[\sin ^{2}\left(2 \theta_{002}\right) * \cos \left(\theta_{002}\right)\right]$, here $\mathbf{L} \mathbf{P}_{\mathbf{0 0 2}}=\mathbf{2 . 8 1}$

Thus, Eq. 3.1B may be re-written as $I_{002}=\{4[(\mathbf{0 . 4} * \mathbf{1 9 . 0 4})+(\mathbf{0 . 6} * \mathbf{6 1 . 6})]\}^{2} * \mathbf{2} * \mathbf{2 . 8 1} \ldots$ (Eq. 3.3)

It may be noted that small variations in the precise value of $2 \theta$ do not cause significant changes in the calculations. 
Dividing Eq. 3.3 by Eq. 3.2 and rearranging, we get $\eta=0.9 * \sqrt{ }\left[\mathbf{I}_{\mathbf{0 0 1}} / \mathbf{I}_{\mathbf{0 0 2}}\right]$, for $\mathrm{L}_{0}$ with composition $\approx \mathbf{C o}_{40} \mathbf{P t}_{60}$. As demonstrated in Appendix 4 , the order parameter, $\eta$, is associated with an accuracy of \pm 0.06 , presumably associated with the curve fitting.

The maximum attainable order parameter $\eta_{\max }=2 * x$ for non-stoichiometric alloys of composition $\mathrm{Co}_{\mathrm{x}} \mathrm{Pt}_{1-\mathrm{x}}$ or $\mathrm{Co}_{1-\mathrm{x}} \mathrm{Pt}_{\mathrm{x}}$, such that $\mathrm{x} \leq 0.5$. Thus, $\eta$ max $=0.83$ for the composition $\mathrm{Co}_{41.7} \mathrm{Pt}_{58.3}$. For the case of the annealing treatment conducted at $700{ }^{\circ} \mathrm{C}$ for 504 hours, we found that calculating the order parameter based on a single peak fit of the superlattice (001) reflection resulted in $\eta=0.86$. Although this was only a small discrepancy, we fit two peaks to the (001) reflection which gave $\eta=0.80$ while additionally allowing us to justify the presence of the $\mathrm{L}_{2}$ phase upon extended annealing, as demonstrated below.

Based on the rapid achievement of peak coercivity at the higher annealing temperature (Figure 3.2a), and also from TEM results, the transformation kinetics were noted to be significantly more rapid at $700{ }^{\circ} \mathrm{C}$ versus $600{ }^{\circ} \mathrm{C}$. $\mathrm{M}_{\mathrm{s}}$ also approached saturation after extended annealing at $700{ }^{\circ} \mathrm{C}$ (Figure 3.2a). Thus, the phase transformation in the $\mathrm{Co}_{41.7} \mathrm{Pt}_{58.3}$ alloy was expected to have progressed to completion upon extended annealing for 504 hours at the higher annealing temperature, i.e, $700{ }^{\circ} \mathrm{C}$. If the sample completely transformed to single-phase $\mathrm{L} 1_{0}$, then all three c-axis variants would be expected to be present in equal volume fractions, when averaged over macroscopic regions.

We know that the tetragonality of the $\mathrm{L} 1_{0}$ unit cell leads to splitting of the $\{200\} /\{002)$ peaks. Then, owing to multiplicity considerations, the intensity ratio $\left(\mathrm{I}_{200}+\mathrm{I}_{020}\right) / \mathrm{I}_{002}$ must theoretically equal 2 for single-phase $\mathrm{L} 1_{0}$. A value exceeding 2 would indicate either incomplete $\mathrm{A} 1 \rightarrow \mathrm{L} 1_{0}$ transformation (which we reject for this sample annealed at $700{ }^{\circ} \mathrm{C}$ for 504 hours), or the additional presence of the $\mathrm{L}_{2}$ phase. From the experimental data presented in Figure 3.6b, we have $\left(I_{200}+I_{020}\right) / I_{002}=2.7$. From the calculations below, this implies that the present material contained $81 \% \mathrm{~L}_{0}$ and $19 \% \mathrm{L1}_{2}$ by volume. We obtained an identical value for the volume fractions from calculations based on fitting the $\{001\}$ superlattice reflection to two peaks attributed to contributions from the $\mathrm{L}_{0}$ and the $\mathrm{L}_{2}$ phases. In this case, the intensity ratio employed was $\mathrm{I}_{\{100\}-\mathrm{L} 12} / \mathrm{I}_{(001) \text { - L10 }}$; and the correct multiplicity factors were accounted for. 


\section{Volume fraction determination using the $\{200\}$ / (002) peaks:}

$$
\left[\left(\mathrm{I}_{200}+\mathrm{I}_{020}\right)_{\mathrm{L} 10} /\left(\mathrm{I}_{200}+\mathrm{I}_{020}\right)_{\mathrm{L} 12}\right]=\mathrm{F}_{\mathrm{L} 10}^{2} \cdot \mathrm{m}_{\mathrm{L} 10} \cdot \mathrm{LP}_{\mathrm{L} 10} /\left(\mathrm{F}_{\mathrm{L} 12}^{2} \cdot \mathrm{m}_{\mathrm{L} 12} \cdot \mathrm{LP}_{\mathrm{L} 12}\right) \ldots(\mathrm{Eq} \cdot 3.4)
$$

The structure factor for fundamental reflections equals $\left[4 *\left(X_{\mathrm{Co}} f_{\mathrm{Co}, 002}+\mathrm{X}_{\mathrm{Pt}} f_{\mathrm{Pt}, 002}\right)\right]$ for non-

stoichiometric $\mathrm{L}_{0}$ as well as $\mathrm{L}_{2}{ }^{44}$. The narrow two-phase region in the Co-Pt phase diagram indicates that there is only a slight difference between the compositions $\left(\mathrm{X}_{\mathrm{Co}}, \mathrm{X}_{\mathrm{Pt}}\right)$ of the $\mathrm{L}_{0}$ and $\mathrm{L}_{2}$ phases obtained upon annealing the $\mathrm{Co}_{41.7} \mathrm{Pt}_{58.3}$ base material at $700{ }^{\circ} \mathrm{C}$ or $600{ }^{\circ} \mathrm{C}$. The $\{200\}$ peaks for both phases are very closely spaced, resulting in similar atomic form factors $\left(\mathrm{f}_{\mathrm{Co}}\right.$ , $f_{\mathrm{Pt}}$ ) for both phases. Thus, $\mathrm{F}_{\mathrm{L} 10}=\mathrm{F}_{\mathrm{L} 12}$.

$\mathrm{LP}_{\mathrm{L} 10}=\mathrm{LP}_{\mathrm{L} 12}$ due to the small difference in peak positions. The multiplicity of the $\{200\}$ reflection is 6 for $\mathrm{L}_{2}$ and 4 for $\mathrm{L} 1_{0}$, owing to tetragonality in the $\mathrm{L} 1_{0}$ phase.

Hence, $\left[\left(I_{200}+I_{020}\right)_{L 10} /\left(I_{200}+I_{020}\right) L_{12}\right]=4 / 6=2 / 3 \ldots($ Eq. 3.5$)$

If volume $\mathrm{V}$ of the $\mathrm{L} 1_{0}$ phase corresponds to an intensity $\left(\mathrm{I}_{200}+\mathrm{I}_{020}\right)_{\mathrm{L} 10}=2 \mathrm{x}$, then, from Eq.3.5, an equal volume $\mathrm{V}$ of $\mathrm{L}_{2}$ corresponds to an intensity $\left(\mathrm{I}_{200}+\mathrm{I}_{020}\right)_{\mathrm{L} 12}=3 \mathrm{x}$.

Hence, a given volume $\mathrm{V}^{\prime}$ of $\mathrm{L}_{2}$ corresponds to an intensity $\left(\mathrm{I}_{200}+\mathrm{I}_{020}\right)_{\mathrm{L} 12}=3 \mathrm{x} * \mathrm{~V}^{\prime} / \mathrm{V}$.

Thus, the ratio of observed intensities may be rewritten in terms of the volumes of the phases as:

$$
\begin{aligned}
& \left(\mathrm{I}_{200}+\mathrm{I}_{020}\right)_{\mathrm{L} 10} /\left(\mathrm{I}_{200}+\mathrm{I}_{020}\right)_{\mathrm{L} 12}=2 /\left[3\left(\mathrm{~V}^{\prime} / \mathrm{V}\right)\right] \\
& \Rightarrow \mathrm{V}^{\prime} / \mathrm{V}=2 /\left[3\left(\left(\mathrm{I}_{200}+\mathrm{I}_{020}\right)_{\mathrm{L} 10} /\left(\mathrm{I}_{200}+\mathrm{I}_{020}\right)_{\mathrm{L} 12}\right)\right] \quad \ldots(\mathrm{Eq} .3 .6)
\end{aligned}
$$

For the sample annealed at $700{ }^{\circ} \mathrm{C}$ for 504 hours:

The observed intensity ratio $\left(\mathrm{I}_{200}+\mathrm{I}_{020}\right)_{\text {total }} / \mathrm{I}_{002}=2.72$

$$
\Rightarrow\left[\left(\mathrm{I}_{200}+\mathrm{I}_{020}\right)_{\mathrm{L} 10}+\left(\mathrm{I}_{200}+\mathrm{I}_{020}\right)_{\mathrm{L} 12}\right] / \mathrm{I}_{002}=2.72 \quad \ldots(\mathrm{Eq} .3 .7)
$$

For the completely ordered $\mathrm{L}_{0}$ phase, $\left(\mathrm{I}_{200}+\mathrm{I}_{020}\right)_{\mathrm{L} 10}=2 * \mathrm{I}_{002}$

From Eq. 3.7 and Eq. 3.8, we get:

$$
\left(\mathrm{I}_{200}+\mathrm{I}_{020}\right)_{\mathrm{L} 12}=0.72 * \mathrm{I}_{002} \quad \ldots(\text { Eq. 3.9) }
$$


Substituting Eq. 3.8 and Eq. 3.9 in Eq. 3.6, $\mathrm{V}^{\prime} / \mathrm{V}=2 * 0.72 /(3 * 2)=0.24 / 1$

$\Rightarrow$ Volume fraction of $L 1_{2}=0.24 /(0.24+1)=0.19$

$\Rightarrow$ Volume fraction of $L 1_{0}=1-0.19=0.81$

The volumes have an associated error of $\pm 4 \%$ as long as the baseline fit is within one fourth of the standard deviation of the average noise, which usually holds true for visually reasonable baseline fits.Visually unreasonable baseline fits are seen to produce larger errors. Only low signal to noise x-ray data may be quantitatively analyzed in a meaningful way. Error fraction estimation associated with volume fraction determination is outlined in Appendix 4.

\section{Volume fraction determination using the $\{100\}_{\mathrm{L} 12} /(001)_{\mathrm{L} 10}$ peaks}

$\left(\mathrm{I}_{001}\right)_{\mathrm{L} 10} /\left(\mathrm{I}_{001}\right)_{\mathrm{L} 12}=\mathrm{F}_{\mathrm{L} 10}^{2} \cdot \mathrm{m}_{\mathrm{L} 10} \cdot \mathrm{LP}_{\mathrm{L} 10} /\left(\mathrm{F}_{\mathrm{L} 12}^{2} \cdot \mathrm{m}_{\mathrm{L} 12} \cdot \mathrm{LP}_{\mathrm{L} 12}\right) \ldots(\mathrm{Eq} \cdot 3 \cdot 10)$

The structure factor for superlattice reflections is $\left[\eta * 2\left(\mathrm{f}_{\mathrm{Pt}, \mathrm{s}}-\mathrm{f}_{\mathrm{Co}, \mathrm{s}}\right)\right]$ for the $\mathrm{L} 1_{0}$ phase, and $\left[\eta *\left(f_{\mathrm{Pt}, \mathrm{s}}-\mathrm{f}_{\mathrm{Co}, \mathrm{s}}\right)\right]$ for the $\mathrm{L}_{2}$ phase ${ }^{44}$. As discussed previously, the compositions $\left(\mathrm{X}_{\mathrm{Co}}, \mathrm{X}_{\mathrm{Pt}}\right)$ of the $\mathrm{L}_{0}$ and $\mathrm{L1}_{2}$ phases under consideration are quite similar. The $\{200\}$ peaks for both phases are very closely spaced, resulting in similar atomic form factors $\left(\mathrm{f}_{\mathrm{Co}}, \mathrm{f}_{\mathrm{Pt}}\right)$ for both phases. Thus, $\mathrm{F}_{\mathrm{L} 10}=2 * \mathrm{~F}_{\mathrm{L} 12} \cdot \mathrm{LP}_{\mathrm{L} 10}=\mathrm{LP}_{\mathrm{L} 12}$ for the closely spaced $\{100\}_{\text {L12 }}$ and (001) L10 peaks. The multiplicity of $\{100\}_{\mathrm{L} 12}$ reflection is 6 , while that of the $(001)_{\mathrm{L} 10}$ reflection is 2 .

Hence, $\left(I_{001}\right)_{L 10} /\left(I_{001}\right)_{L 12}=2^{2} * 2 / 6=4 / 3$

If volume $\mathrm{V}$ if $\mathrm{L} 1_{0}$ corresponds to an intensity $\left(\mathrm{I}_{001}\right)_{\mathrm{L} 10}=4 \mathrm{x}$, then, from Eq. 3.11, an equal volume $\mathrm{V}$ of $\mathrm{L}_{2}$ corresponds to an intensity $\left\{\mathrm{I}_{100}\right\}_{\mathrm{L} 12}=3 \mathrm{x}$.

Hence, a given volume $\mathrm{V}^{\prime}$ of $\mathrm{L}_{2}$ corresponds to an intensity $\left\{\mathrm{I}_{100}\right\}_{\mathrm{L} 12}=3 \mathrm{x} * \mathrm{~V}^{\prime} / \mathrm{V}$.

Thus, the ratio of observed intensities may be rewritten in terms of the volumes of the phases as:

$$
\begin{aligned}
& \Rightarrow\left(\mathrm{I}_{001}\right)_{\mathrm{L} 10} /\left\{\mathrm{I}_{100}\right\}_{\mathrm{L} 12}=4 /\left[3\left(\mathrm{~V}^{\prime} / \mathrm{V}\right)\right] \\
& \Rightarrow \mathrm{V}^{\prime} / \mathrm{V}=4 /\left[3\left(\mathrm{I}_{001}\right)_{\mathrm{L} 10} /\left\{\mathrm{I}_{100}\right\}_{\mathrm{L} 12}\right]
\end{aligned}
$$


For the sample annealed at $700{ }^{\circ} \mathrm{C}$ for 504 hours:

The $1^{\text {st }}$ peak $\left(\right.$ at $2 \theta \approx 23.5^{\circ}$ ) corresponds to $\mathrm{LI}_{2}$ and the second peak (at $2 \theta \approx 23.7^{\circ}$ ) corresponds to $\mathrm{L} 1_{0}$ based on expected lattice parameters of the two phases.

The observed value of $\left(\mathrm{I}_{001}\right)_{\mathrm{L} 10} /\left\{\mathrm{I}_{100}\right\}_{\mathrm{L} 12}=1170 / 204$ and substituting in Eq. 3.12, $\mathrm{V}^{\prime} / \mathrm{V}=$ $0.23 / 1$.

$\Rightarrow$ Volume fraction of $\mathrm{LI}_{2}=0.23 /(0.23+1)=0.19$

$\Rightarrow$ Volume fraction of $\mathrm{LI}_{0}=0.81$

From these calculations, that the volume fractions calculated from the split $\{100\} /(001)$ peaks and from the split 200/002 peaks for the sample annealed at $700{ }^{\circ} \mathrm{C}$ for 504 hours sample are equal. This sample is then fully transformed and consists of $19 \% \mathrm{~L}_{2}$ and $81 \% \mathrm{~L}_{0}$ by volume.

Lattice parameters obtained from $\{311\}$ peaks and order parameter, obtained from the analysis of this fully ordered transformed sample are summarized in Table 3.2. There is an error of \pm $0.006 \AA$ associated with lattice parameter determination based on the nature of the peak fit.

\begin{tabular}{|c|c|c|c|c|}
\hline & $\eta$ & $\mathbf{c}(\mathbf{A})$ & $\mathbf{a}(\mathbf{A})$ & $\mathbf{c / a}$ \\
\hline $\begin{array}{c}\mathrm{L}_{0} \text { from sample annealed at } \\
700{ }^{\circ} \mathrm{C} \text { for } 504 \mathrm{hrs} .\end{array}$ & 0.86 & 3.773 & 3.818 & 0.988 \\
\hline
\end{tabular}

Table 3.2: Results from quantitative $x$-ray analysis of fully transformed $\mathrm{Co}_{41.7} \mathrm{Pt}_{58.3}$ sample. .

The sample annealed for an extended duration at $600{ }^{\circ} \mathrm{C}$, for 720 hours, is similarly analyzed below. As in the case of the sample annealed at $700{ }^{\circ} \mathrm{C}$ for an extended time period, we fit the '(001)' reflection to two peaks: $\left(\mathrm{I}_{001}\right)_{\mathrm{L} 10}$ and $\left\{\mathrm{I}_{100}\right\}_{\mathrm{L12}}$. We assumed that the second peak was associated with the $\mathrm{L}_{0}$ phase, as was previously justified. Corresponding calculations indicated a physically reasonable $\mathrm{L} 1_{0}$ order parameter of 0.88 , which, accounting for the curve fitting dependent- error in $\eta$, implies complete ordering of the $L 1_{0}$ phase. In contrast, a single-peak fit to the (001) reflection yielded an order parameter of 1.01, greater than the maximum attainable 
value for this composition. Volume fraction calculations shown below indicate the presence of $72 \% \mathrm{L1}_{0}, 7 \% \mathrm{L1}_{2}$ and a significant amount of untransformed $\mathrm{A} 1,21 \%$, after annealing at $600{ }^{\circ} \mathrm{C}$ for 720 hours.

Volume fraction determination using the $\{100\}_{\mathrm{L} 12} /(001)_{\mathrm{L10}}$ peaks

For the sample annealed at $600{ }^{\circ} \mathrm{C}$ for 720 hours:

If the $1^{\text {st }}$ peak $\left(\right.$ at $2 \theta \approx 23.5^{\circ}$ ) corresponds to $L 1_{2}$ and the second peak (at $2 \theta \approx 23.7^{\circ}$ ) corresponds to $\mathrm{Ll}_{0}$.

The observed value of $\left(\mathrm{I}_{001}\right)_{\mathrm{L} 10} /\left\{\mathrm{I}_{100}\right\}_{\mathrm{L} 12}=1258 / 88$ and substituting in Eq. 3.12, $\mathrm{V}^{\prime} / \mathrm{V}=0.09 / 1$.

$\Rightarrow$ Volume fraction of $\mathrm{LI}_{2}=1.56 /(1.56+1)=0.09$

$\Rightarrow$ Volume fraction of $\mathrm{LI}_{0}=0.91$

Volume fraction determination using the $\{200\}$ / (002) peaks:

For the sample annealed at $600^{\circ} \mathrm{C}$ for 720 hours:

The observed intensity ratio $\left(\mathrm{I}_{200}+\mathrm{I}_{020}\right)_{\text {total }} / \mathrm{I}_{002}=3.17$

$\left[\left(\mathrm{I}_{200}+\mathrm{I}_{020}\right)_{\mathrm{L} 10}+\left(\mathrm{I}_{200}+\mathrm{I}_{020}\right)_{\mathrm{L} 12}\right] / \mathrm{I}_{002}=3.17 \quad \ldots($ Eq. 3.13)

For the completely ordered $\mathrm{L}_{0}$ phase, $\left(\mathrm{I}_{200}+\mathrm{I}_{020}\right)_{\mathrm{L} 10}=2 * \mathrm{I}_{002}$

From Eq. 3.8 and Eq. 3.13, we get:

$\left(\mathrm{I}_{200}+\mathrm{I}_{020}\right)_{\mathrm{L} 12}=1.17 * \mathrm{I}_{002}$

Substituting Eq. 3.8 and Eq. 3.14 in Eq. 3.6, $\mathrm{V}^{\prime} / \mathrm{V}=2 * 1.17 /(3 * 2)=0.39 / 1$

$\Rightarrow$ Volume fraction of $\mathrm{LI}_{2}=0.39 /(0.39+1)=0.28$

$\Rightarrow$ Volume fraction of $\mathrm{LI}_{0}=0.72$

The calculations from $\{100\}_{\mathrm{L} 12} /(001)_{\mathrm{L} 10}$ peak and $\{200\} /(002)$ analyses appear inconsistent but were based on the assumption that the sample was completely transformed and contained 
only the $\mathrm{L}_{0}$ and $\mathrm{L1}_{2}$ phases at this stage. However, it is highly likely that residual A1 was also present which may be accounted for as follows:

$\left[\left(\mathrm{I}_{200}+\mathrm{I}_{020}\right)_{\mathrm{L} 10}+\left(\mathrm{I}_{200}+\mathrm{I}_{020}\right)_{\mathrm{soft}}\right] / \mathrm{I}_{002}=3.17 \quad \ldots$ (Eq. 3.13)

Where $\left(\mathrm{I}_{200}+\mathrm{I}_{020}\right)_{\mathrm{soft}}=\left(\mathrm{I}_{200}+\mathrm{I}_{020}\right)_{\mathrm{A} 1}+\left(\mathrm{I}_{200}+\mathrm{I}_{020}\right)_{\mathrm{L} 12}$

For the completely ordered $\mathrm{L1}_{0}$ phase, $\left(\mathrm{I}_{200}+\mathrm{I}_{020}\right)_{\mathrm{L} 10}=2 * \mathrm{I}_{002}$

From Eq. 3.8 and Eq. 3.13, we get:

$\left(\mathrm{I}_{200}+\mathrm{I}_{020}\right)_{\mathrm{soft}}=1.17 * \mathrm{I}_{002} \quad \ldots$ (Eq. 3.14a)

Substituting Eq. 3.8 and Eq. 3.14 in Eq. 3.6, V'"/V =2*1.17/(3*2) =0.39/1

where $\mathrm{V}^{\prime \prime}$ is the volume of the soft phase.

$\Rightarrow$ Volume fraction of soft phase $=0.39 /(0.39+1)=0.28$

$\Rightarrow$ Volume fraction of $\mathrm{LI}_{0}=0.72$

Of the soft phase, 9/91*.72=0.07 is L12 and $0.28-0.07=0.23$ is A1.

Furthermore, at this stage of intermediate phase transformation, as well as for the peak hardness sample annealed at $600{ }^{\circ} \mathrm{C}$ for 96 hours, strain broadening of peaks impedes rigorous determination of lattice parameters for these samples. The low tetragonality of the $\mathrm{L}_{0}$ phase, i.e., absence of split fundamental reflections also prevents volume fraction and order parameter estimation in the peak hardness sample. An estimation of lattice strain is shown in Appendix 5. While the linear dependence of lattice parameter on strain results in a dramatic impact on the lattice parameter, strain broadening does not have a significant influence on the Scherrer particle size, as shown below. Thus, the (001) peaks from these samples may still be used to estimate the Scherrer particle size.

Scherrer particle size $\Lambda=0.9 \lambda / \mathcal{B} \cos (\theta)$

...(Eq.3.15) 
where $\lambda$ is the incident $\mathrm{x}$-ray wavelength $=0.154 \mathrm{~nm}, \mathcal{B}$ is the peak width at half the maximum intensity and $\theta$ is the peak position (FWHM).

Accounting for instrumental and strain broadening, we have:

$\mathcal{B}_{\text {corrected }}=\sqrt{ }\left(\mathcal{B}_{\text {measured }}^{2}-\mathcal{B}_{\text {standard }}{ }^{2}-\mathcal{B}_{\text {strain }}{ }^{2}\right)$

where $\mathcal{B}_{\text {measured }}$ is the experimental peak width at half the maximum intensity FWHM, $\mathcal{B}_{\text {standard }}$ is the peak width associated with instrumental broadening and $\mathcal{B}_{\text {strain }}$ is the strain broadening. Eq. 3.16 is appropriate to Gaussian-shaped peaks, which we have used to fit the partially transformed samples due to the large degree of broadening. The uncorrected and uncorrected Scherrer sizes from samples annealed for extended durations at 700 and $600{ }^{\circ} \mathrm{C}$ as well as the peak coercivity sample annealed for 96 hours at $600{ }^{\circ} \mathrm{C}$ are tabulated in Table 3.3. Scherrer particle size depends on the curve fitting and the corresponding error is estimated to be $\pm 2 \mathrm{~nm}$.

\begin{tabular}{|l|l|l|}
\hline Sample & Scherrer size (uncorrected) & $\begin{array}{l}\text { Scherrer particle size } \\
\text { (corrected) }\end{array}$ \\
\hline $600 / 96$ & 11 & 12 \\
\hline $600 / 720$ & 13 & 14 \\
\hline $700 / 504$ & 126 & 228 \\
\hline
\end{tabular}

Table 3.3: Scherrer particle size of key $\mathrm{Co}_{41.7} \mathrm{Pt}_{58.3}$ samples

\section{2. $\mathrm{CO}_{37.6} \underline{\mathrm{Pt}_{62.4}}$}

The XRD patterns from the $\mathrm{Co}_{37.6} \mathrm{Pt}_{62.4}$ alloy samples annealed at $600{ }^{\circ} \mathrm{C}$ and $700{ }^{\circ} \mathrm{C}$ for extended durations are shown in Figure 3.8. While superlattice reflections are clearly seen, the $\{200\} /$ (002) peak splitting was absent at all stages of annealing, indicating an absence of tetragonal distortion. Consequently, we conclude that ordering involves the $\mathrm{A} 1 \rightarrow \mathrm{L}_{2}$ transformation in this alloy, i.e., there is no $\mathrm{L1}_{0}$ phase. This conclusion was further verified by the soft magnetic properties consistently displayed by this material, even post-annealing. 


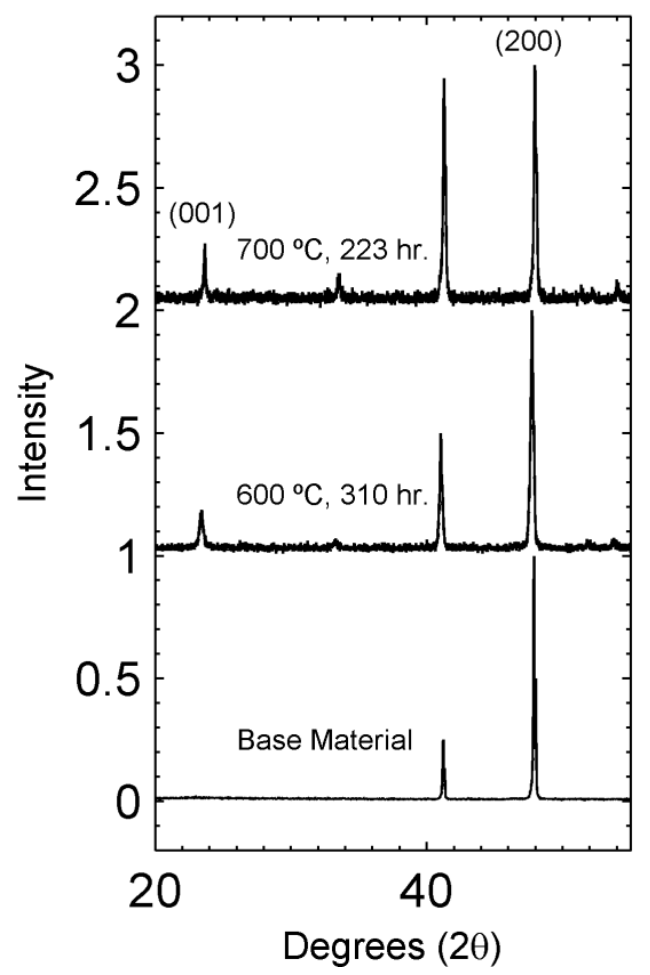

Figure 3.8: $\mathrm{XRD}$ spectra from the $\mathrm{Co}_{37.6} \mathrm{Pt}_{62.4}$ alloy: (a) the base material; samples annealed at (b) $600{ }^{\circ} \mathrm{C}$ for $310 \mathrm{hr}$., and (c) $700{ }^{\circ} \mathrm{C}$ for $233 \mathrm{hr}$.

\begin{tabular}{|c|c|}
\hline Phase/Sample & Lattice Parameter $(\AA)$ \\
\hline $\mathrm{L}_{0}$ from $\mathrm{Co}_{41.7} \mathrm{Pt}_{58.3}$ annealed at 700 for $504 \mathrm{hrs.}$ & $\mathrm{c}=3.773, \mathrm{a}=3.818$ \\
\hline $\mathrm{A} 1$ from $\mathrm{Co}_{41.7} \mathrm{Pt}_{58.3}$ base material & 3.79 \\
\hline $\mathrm{L}_{2}$ from $\mathrm{Co}_{37.6} \mathrm{Pt}_{62.4}$ annealed at 700 for $223 \mathrm{hrs}$. & 3.813 \\
\hline $\mathrm{A} 1$ from $\mathrm{Co}_{37.6} \mathrm{Pt}_{62.4}$ base material & 3.804 \\
\hline
\end{tabular}

Table 3.4: Lattice parameters of off-stoichiometric $\mathrm{L} 1_{0}, \mathrm{~L} 1_{2}$ phases in the vicinity of the twophase coexistence region, as well as corresponding A1 base materials. 
Table 3.4 presents the lattice parameters for the nearly strain-free off-stoichiometric $\mathrm{L}_{0}, \mathrm{~L}_{2}$ phases from the $\mathrm{Co}_{41.7} \mathrm{Pt}_{58.3}$ and $\mathrm{Co}_{37.6} \mathrm{Pt}_{62.4}$ alloys respectively; and for the corresponding $\mathrm{A} 1$ base materials.

We have calculated the order parameter of the $\mathrm{L}_{2}$ phase for the samples annealed for extended durations at both temperatures by comparing the integrated intensities of the $\{100\}$ superlattice and 200) fundamental peaks obtained from fitting analysis of the data in Figure 3.8, as shown below. This eliminated any influence of texture on the relative peak intensities, and hence, on the order parameter calculations. Note, that we could comfortably fit the $\{001\}$ reflection using one peak instead of two, which yielded a better fit in this case. As discussed previously, the $\{100\}$ superlattice reflection can arise only from the $\mathrm{L}_{2}$ phase, but contains no contribution from the disordered phase, being a forbidden reflection for FCC. On the other hand, the $\{200\}$ reflection can arise from both the ordered as well as disordered phases. ${ }^{44}$

\section{$\underline{\text { Order parameter calculation for non-stoichiometric } \mathrm{L}_{2}}$}

$\mathbf{I}_{\mathrm{s}}=\mathbf{F}_{\mathrm{s}}{ }^{2} \cdot \mathbf{m}_{\mathrm{s}} \cdot \mathbf{L} \mathbf{P}_{\mathrm{s}} \quad \ldots(\mathrm{Eq} \cdot 3 \cdot 1 \mathrm{~A})$

$\left.F_{s}=\eta \cdot\left(f_{P t, s}-f_{C o, s}\right)\right]^{44} ;$

For the $\{100\}$ superlattice reflection occuring at $2 \theta_{100} \approx 23.7^{\circ}$,

$$
F_{100}=s .\left(f_{P t}, 100-f_{C o, 100}\right) \text {; }
$$

For $\left[\sin \left(\theta_{100}\right)\right] / \lambda=[\sin (23.7 / 2)] / 1.54 \AA, f_{P t, 100}=71.84$ and $f_{C o, 100}=23.64$;

$$
\mathbf{F}_{100}=\eta *(71.84-23.64) .
$$

$\mathbf{m}_{100}=6$.

$\mathrm{LP}_{100}=\left[1+\cos ^{2}\left(2 \theta_{100}\right)\right] /\left[\sin ^{2}\left(2 \theta_{100}\right) * \cos \left(\theta_{100}\right)\right] ;$ here $\mathbf{L P} \mathbf{P}_{100}=\mathbf{1 1 . 6 3}$

Thus, Eq. 3.1A may be re-written as $\mathbf{I}_{100}=[\eta *(71.84-23.64)]^{2} * 6 * 11.63 \ldots$ (Eq. 3.15)

$\mathbf{I}_{\mathbf{f}}=\mathbf{F}_{\mathrm{f}}{ }^{2} \cdot \mathbf{m}_{\mathbf{f}} \cdot \mathbf{L} \mathbf{P}_{\mathrm{f}} \quad$ WEq. $3 \cdot 1 \mathrm{~B}$

For the $\{200\}$ fundamental reflection occuring at $2 \theta=48.4^{\circ}$, $\mathrm{F}_{\mathrm{f}}=4\left(\mathrm{X}_{\mathrm{Co}} \mathrm{f}_{\mathrm{Co}, 200}+\mathrm{X}_{\mathrm{Pt}} \mathrm{f}_{\mathrm{Pt}, 200}\right), \mathrm{X}$ being the mole fraction. ${ }^{44}$ Here, $\mathrm{X}_{\mathrm{Co}} \approx 0.4, \mathrm{X}_{\mathrm{Co}} \approx 0.6$. 
For $\left[\sin \left(\theta_{200}\right)\right] / \lambda=[\sin (48.4 / 2)] / 1.54 \AA, f_{\mathrm{Pt}, 200}=61.6$ and $\mathrm{f}_{\mathrm{Co}, 200}=19.04$;

$$
\Rightarrow F_{200}=4[(0.4 * 19.04)+(0.6 * 61.6)] .
$$

$\mathbf{m}_{200}=6$.

$\mathbf{L P}_{200}=\left[1+\cos ^{2}\left(2 \theta_{200}\right)\right] /\left[\sin ^{2}\left(2 \theta_{200}\right) * \cos \left(\theta_{200}\right)\right]$, here $\mathbf{L} \mathbf{P}_{200}=\mathbf{2 . 8 1}$

Thus, Eq. 3.1B may be re-written as $\mathrm{I}_{200}=\{\mathbf{4}[(\mathbf{0 . 4} * \mathbf{1 9 . 0 4})+(\mathbf{0 . 6} * \mathbf{6 1 . 6})]\}^{2} * \mathbf{6} * \mathbf{2 . 8 1} \ldots$ Eq. 3.16

Dividing Eq. 3.16 by Eq. 3.15 and rearranging, we get $\eta=\mathbf{1 . 8 1} * \sqrt{ }\left[\mathbf{I}_{\mathbf{0 0 1}} / \mathbf{I}_{\mathbf{2 0 0}}\right]$, for the case of non-stoichiometric $\mathrm{L}_{2}$.

Theoretically, the maximum possible order parameter for an alloy of composition $\mathrm{Co}_{37.6} \mathrm{Pt}_{62.4}$ is $2 * 0.376=0.75$. While the sample may contain a range of order parameters at this stage, calculations based on XRD results indicated an average order parameter of 0.78 for the $\mathrm{L}_{2}$ phase in the sample annealed at $700{ }^{\circ} \mathrm{C}$ for 223 hours, quite close to the theoretical maximum. The superlattice (001) reflection does not present a strong case for 2-peak fitting, nor is there any evidence for (200) / (002) peak splitting after this prolonged aging treatment at $700{ }^{\circ} \mathrm{C}$. Thus, the additional presence of the ordered $\mathrm{L}_{0}$ phase is not indicated. We may argue that the $\mathrm{FCC} \rightarrow \mathrm{L}_{2}$ phase transformation is complete and that the resulting $\mathrm{L}_{2}$ phase is completely ordered upon annealing for 223 hours at $700{ }^{\circ} \mathrm{C}$. As with the case for the $\mathrm{Co}_{41.7} \mathrm{Pt}_{58.3}$ alloy, calculations based on XRD results for samples that underwent extended annealing at $600{ }^{\circ} \mathrm{C}$ suggested an average order parameter of 0.85 , higher than the maximum attainable. As discussed previously, there is no evidence for the $\mathrm{L}_{0}$ phase, even after extended annealing at higher annealing temperatures. However, it is possible that the presence of hybrid ordering phases may impede an accurate quantitative analysis at this stage of incomplete phase transformation.

Order parameter calculations based on fundamental peaks occurring at larger $2 \theta$ values and superlattice reflections occurring at lower $2 \theta$ values are known to result in greater accuracy. ${ }^{44}$ We have used the (001) superlattice reflection for the purpose of our calculations, in keeping with this recommendation, although the fundamental reflection employed was the (002). Were we to use a higher fundamental reflection, such as the (113), instead of the (002) reflection, we would no longer be able to overlook the impact of texture on peak intensities. 


\subsection{Microstructure and Grain Size}

We investigated aged $\mathrm{Co}_{41.7} \mathrm{Pt}_{58.3}$ samples exhibiting the most intriguing magnetic properties using conventional TEM. The time evolution of the transformation microstructures was seen to fully agree with predominant ordering to the $\mathrm{L}_{0}$ phase, confirming the results from X-Ray analysis. Figure 3.9 shows the microstructures and length-scales for samples aged at different temperatures and for different durations, and hence, at different stages of phase transformation.

Figure $3.9(\mathrm{a}, \mathrm{d})$ reveal the microstructure of the alloy resulting from an annealing treatment at $600{ }^{\circ} \mathrm{C}$ for 96 hours, in Bright Field (BF) and Dark Field (DF) modes respectively. At this stage of phase transformation, the sample displayed tweed contrast on a fine length-scale of 15-20 nm. Tweed is a manifestation of strain contrast associated with coherently-supported tetragonal distortions caused by interleaved c-axis orientations of the $\mathrm{L} 1_{0}$ phase, lying on (011) and (0 1 -1) habit planes within the untransformed disordered FCC matrix ${ }^{22,24}$. 
(a)
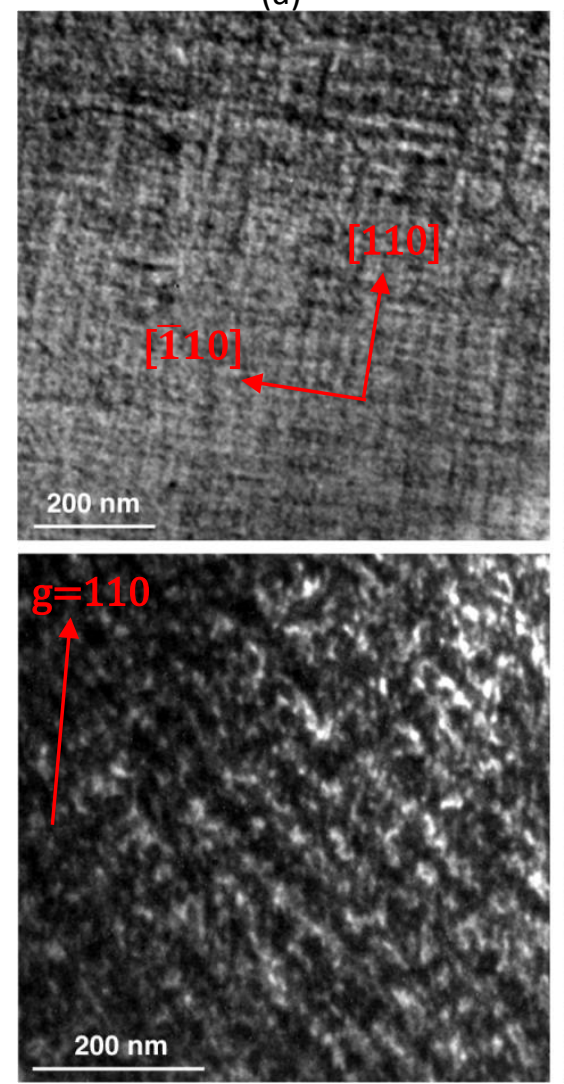

(d) (b)
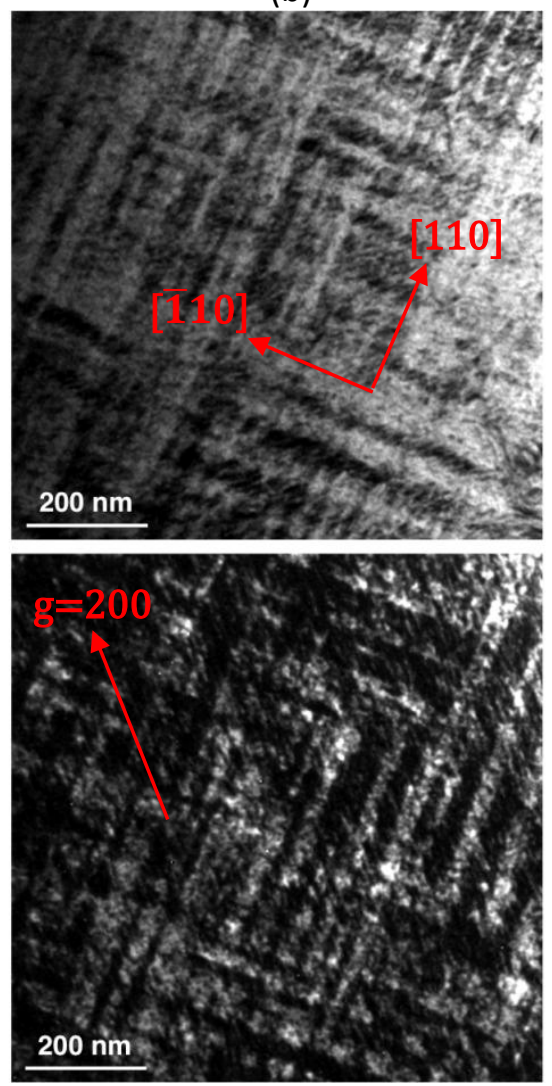

(e) (c)
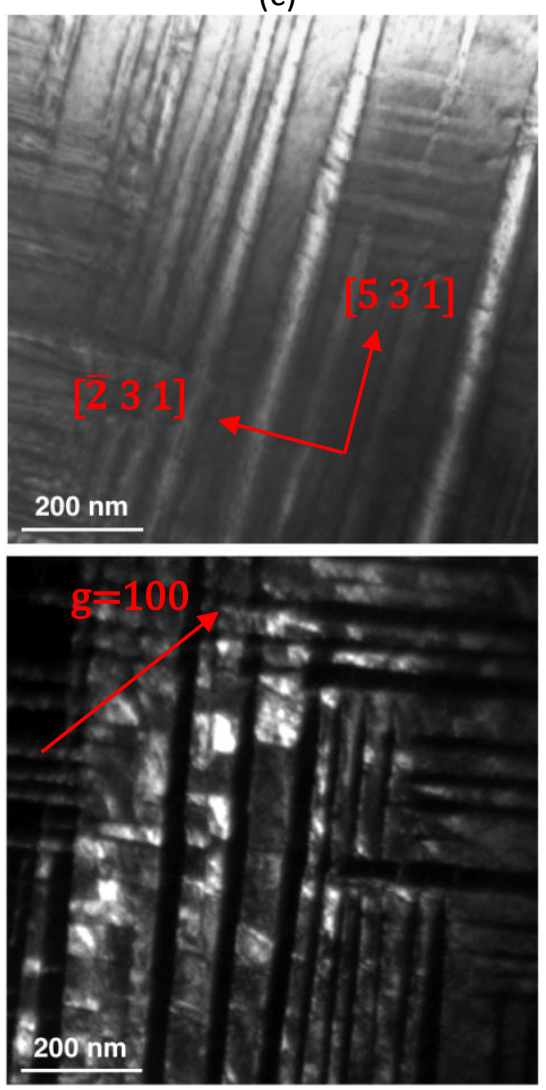

(f)

Figure 3.9: (a-c) Bright Field and (d,e) Dark Field (bottom row) and (f) Dirty Dark Field TEM micrographs for $\mathrm{Co}_{41.7} \mathrm{Pt}_{58.3}$ samples annealed at (a,d) $600{ }^{\circ} \mathrm{C}$ for 96 hours; (b,e) $600{ }^{\circ} \mathrm{C}$, for 720 hours; and (c,f) $700{ }^{\circ} \mathrm{C}$ for 24 hours. All images are along the <001> zone axis except for (c), which is along the $\langle 310>$ zone axis. The Bright Field images are all on the same length scale to facilitate a direct comparison.Corresponding crystal directions and $\mathbf{g}$ vectors are shown.

Linear intercept analysis was performed on micrographs of these samples to obtain corresponding mean microstructural lengthscales, $\lambda^{\prime}$, as shown in Appendix.5. $\lambda^{\prime}$ is measured as the periodicity of alternating contrast, as indicated in Fig. 3.10. The results are summarized in Table 3.5. 


\begin{tabular}{|c|c|}
\hline $\mathbf{C o}_{\mathbf{4 1 . 7}} \mathbf{P t}_{\mathbf{5 8 . 3}}$ sample & Microstructural lengthscale: $\boldsymbol{\lambda}^{\prime}(\mathbf{n m})$ \\
\hline $600{ }^{\circ} \mathrm{C}$ for $96 \mathrm{hrs}$. (peak hardness) & $12 \pm 2$ \\
\hline $600{ }^{\circ} \mathrm{C}$ for 720 hrs. & $23 \pm 5$ \\
\hline $700{ }^{\circ} \mathrm{C}$ for 24 & $38 \pm 13$ \\
\hline
\end{tabular}

Table 3.5: Microstructural lengthscales for key samples

The Scherrer particle size for the peak coercivity sample and the structural periodicity obtained from TEM agree very well for the peak hardness sample annealed at $600{ }^{\circ} \mathrm{C}$ for 96 hours. The coercivity of this sample will be quantitatively modeled in the next section. For the coarsened sample obtained from annealing at $600{ }^{\circ} \mathrm{C}$ for 720 hours, the TEM structural periodicity obtained from intercept analysis, $23 \mathrm{~nm}$, is larger than the Scherrer particle size, $14 \mathrm{~nm}$. This may be because particles have begun to coalesce by this stage, forming bands of alternate c variants, as shown in Figure 3.10. Thus, XRD analysis gives a measure of the particle size whereas TEM provides an estimate of the width of the incipient band.

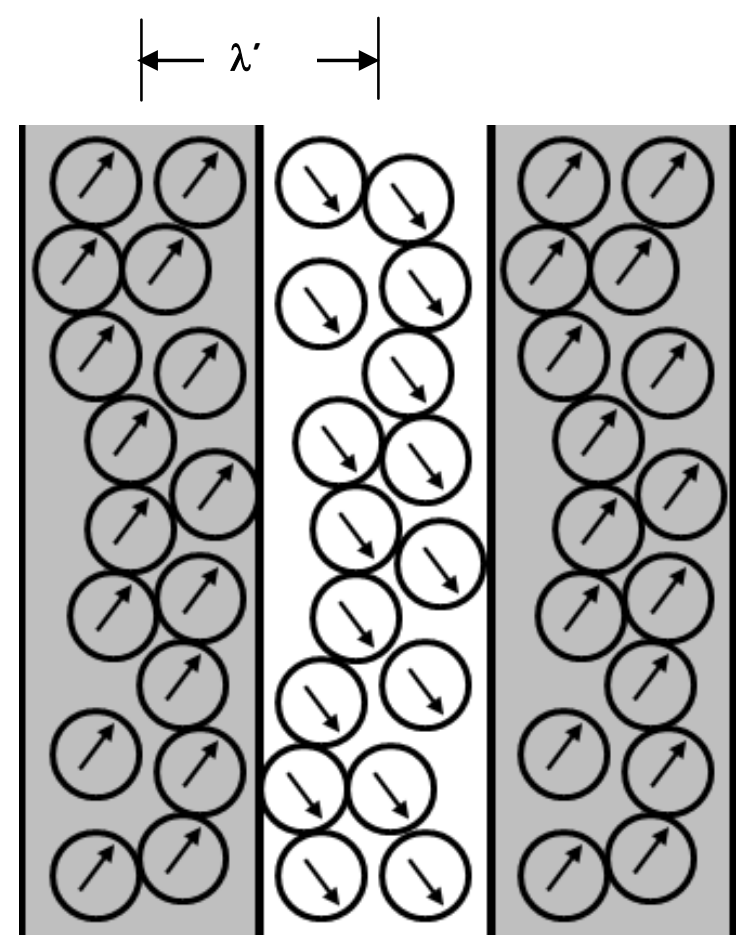

Figure 3.10: Schematic microstructure in $\mathrm{Co}_{41.7 .} \mathrm{Pt}_{58.3}$ sample annealed at $600{ }^{\circ} \mathrm{C}$ for 720 hours. 
From Figure 3.11a, we see that the <001> Selected Area Diffraction Pattern (SADP) for this peak hardness sample aged at $600{ }^{\circ} \mathrm{C}$ for 96 hours contains the (100), (010) as well as the (110) superlattice reflections, indicated by red circles. A schematic representation of this diffraction pattern is shown in Figure 3.11b wherein red and black circles have been used to identify the superlattice and fundamental reflections, respectively. The simultaneous presence of the abovementioned superlattice reflections can arise either from the presence of particles of all three c-axis variants of the $\mathrm{L} 1_{0}$ phase or alternately, from the presence of $\mathrm{L}_{2}$ particles. As can be seen from Table 3.1, the coercivity of the $\mathrm{Co}_{37.6} \mathrm{Pt}_{62.4}$ sample subjected to an identical aging treatment at $600{ }^{\circ} \mathrm{C}$ for 96 hours is significantly low, and explained by the presence of the $\mathrm{L}_{2}$ phase in this sample. However, the observed high coercivity in the peak hardness $\mathrm{Co}_{41.7} \mathrm{Pt}_{58.3}$ sample cannot be explained by the $\mathrm{L}_{2}$ phase nor does our XRD analysis support its existence. Hence, we rule out the formation and presence of the $\mathrm{L1}_{2}$ phase and conclude that at this early stage in phase transformation corresponding to peak coercivity and maximum exchange-coupling, the alloy is comprised of very small $\mathrm{L}_{0}$ particles embedded in an FCC matrix.
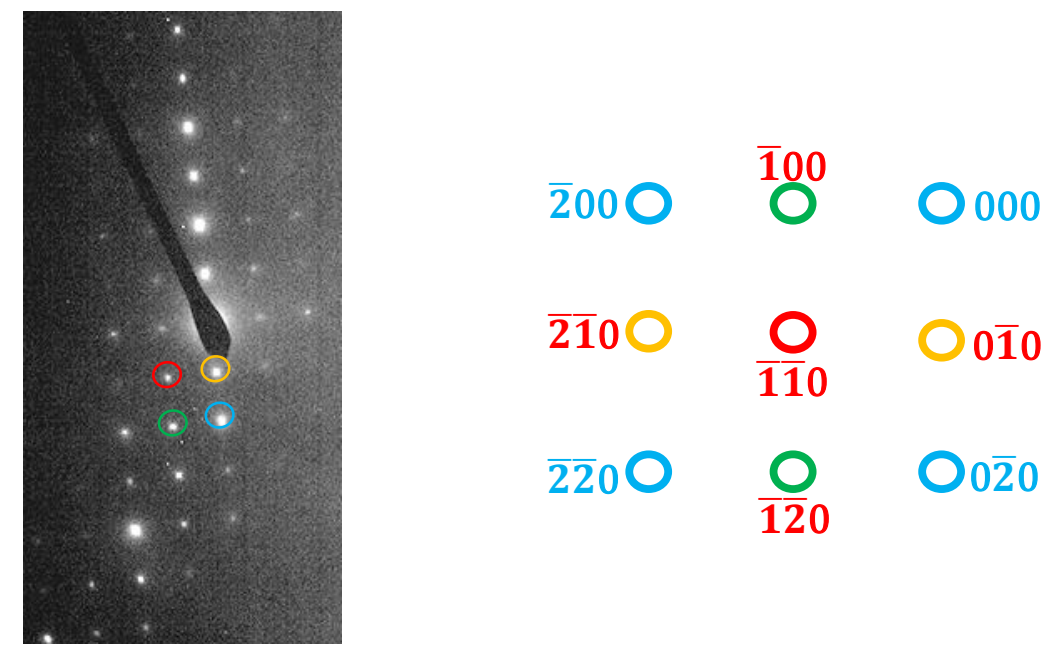

Figure 3.11: (a) Selected area diffraction pattern along the [001] zone axis for the peak hardness sample annealed at $600{ }^{\circ} \mathrm{C}$ for 96 hours. Key superlattice reflections are identified using red circles. The pointer is over the zero beam. (b) Schematic representation of the (001) diffraction pattern. Red circles indicate 110 type superlattice reflections, yellow circles indicate 010 type superlattice reflections, green circles indicate 100 type superlattice reflections and blue circles indicate fundamental reflections. 
An extended annealing time of 720 hours at this temperature led to a better organization of the $\mathrm{L1}_{0}$ particles into lamellae, as shown in Figure 3.9 (b, e) using BF and DF imaging techniques, respectively. However, dark-field microscopy clearly indicates that the spacing between the lamellae is only 20-40 nm, suggesting that the dimensions of particles within these lamella would be of that order, if not lower. With respect to Figure 3.11, the SADP of the sample annealed at $600{ }^{\circ} \mathrm{C}$ for 720 hours contains the (100) and (010) superlattice reflections while the (110) reflection is missing. This of course ruled out the possible presence of $\mathrm{L}_{2}$ at this stage. We thus conclude that two of the three $\mathrm{L1}_{0}$ variants grew at the cost of the third, upon annealing beyond 96 hours at 600 C. By 720 hours, the third variant did not exist in any detectable quantity.

On the other hand, aging at $700{ }^{\circ} \mathrm{C}$ for only 24 hours produced the polytwinned structure comprising coarsened plates of the $\mathrm{L} 1_{0}$ phase, as shown in Figure 3.9c. The mutually aligned plates were associated with one of two $\mathrm{L}_{0}$ orientation variants, as confirmed by the DF image in Figure 3.9f. Anti-phase domain boundaries were clearly present within the coarsened plates, at this stage in the phase transformation.

In comparison, the $\mathrm{BF}$ image obtained from the sample step-cooled from $740{ }^{\circ} \mathrm{C}$ to $700{ }^{\circ} \mathrm{C}$ at the rate of $10{ }^{\circ} \mathrm{C}$ per 12 hours and then held at $700{ }^{\circ} \mathrm{C}$ for 72 hours produces a considerably macrotwinned structure, as shown in Figure 3.12. The higher density of pinning sites likely results in high coercivity in this sample. 


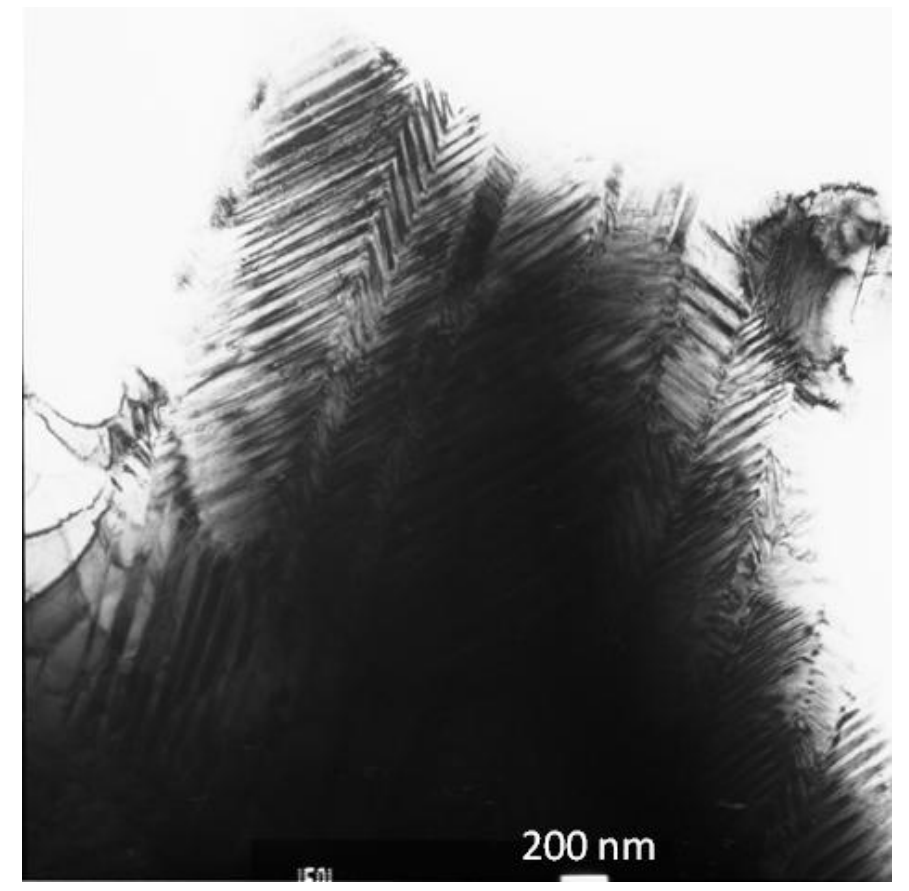

Figure 3.12: Bright field TEM image from sample which underwent the sequenced heat treatment.

Grain sizes were determined to range from $21-28 \mu \mathrm{m}$ from optical metallography, using the mean intercept method. The grain sizes were likely set by the homogenization anneals.

\section{B. Discussion:}

Chemical ordering in eutectoid and near-eutectoid Co-Pt alloys can produce strongly exchangecoupled hard/soft magnetic nanocomposites consisting of interleaved $\mathrm{L}_{0}$ and $\mathrm{L}_{2}$ phases in the self-assembled, highly regular 'nanochessboard' structure. The structure - magnetic property relationships in this novel eutectoid microstructure are unexplored.

We have characterized the non-stoichiometric ordered phases occurring at the boundaries of the $\mathrm{L}_{0}+\mathrm{L}_{2}$ two-phase region, which provides a framework for the study of eutectoid alloys described in Chapter 4, using X-Ray Diffraction, Vibrating Sample Magnetometry and 
Transmission Electron Microscopy. The present study helps establish the behavior for the $\mathrm{L} 1_{0}$ and $\mathrm{L}_{2}$ phases at compositions near the two-phase boundaries. This will allow us to understand the baseline properties of the constituent phases of the two-phase chessboard region on the phase diagram. Specifically, we have examined the disorder - order phase transformation: its kinetics, degree of order, the saturation magnetization; magnetic properties viz., coercivity, remanence, remanence ratio and the Curie temperatures. This study has additionally uncovered interesting and complex magnetic age hardening behavior as a function of time and temperature, corresponding to accompanying transformation in microstructural lengthscales, order parameters, phases and phase fractions. We will now discuss the phase transformation, the presence of exchange coupling, and domain wall pinning to describe the nature of magnetization reversal.

The $\mathrm{A} 1 \rightarrow \mathrm{L1}_{0}$ transition for the $\mathrm{Co}_{41.7} \mathrm{Pt}_{58.3}$ sample proceeds slowly at $600{ }^{\circ} \mathrm{C}$, where even after an annealing time of 720 hours, XRD suggests that only partial transformation has occurred; and the microstructural lengthscale is still $\approx 23 \mathrm{~nm}$. Annealing at $600{ }^{\circ} \mathrm{C}$ for 96 hours produced simultaneously the highest coercivity, $4 \mathrm{kOe}$, and remanence ratio, 0.8. TEM indicates that after 96 hours, the sample is still in the early stages of the transformation, based on the observation of tweed contrast with a quasi-periodicity of $12 \mathrm{~nm}$. Annealing at $700{ }^{\circ} \mathrm{C}$ results in a significantly rapid transition, with a peak coercivity of 2500 Oe after only $2.5 \mathrm{hr}$. annealing, and a microstructure comprising of coarse $\mathrm{L} 1_{0}$ plates, formed after only 24 hours annealing.

After 504 hours at $700{ }^{\circ} \mathrm{C}$, based on the evidence of the intensity ratios of the split $\{200\} /(002)$ peaks (further corroborated by calculations based on the relative intensities of the split (001) peak), we conclude that the $\mathrm{L}_{0}$ and $\mathrm{L}_{2}$ phases co-exist after this extended annealing period. It is a reasonable assumption that the volume fractions determined for the two phases: $81 \% \mathrm{~L} 1_{0}$ and $19 \% \mathrm{~L}_{2}$ by volume, are equilibrium values, given the extended annealing time. It therefore follows, using the lever rule, that for a composition $\mathrm{Co}_{41.7} \mathrm{Pt}_{58.3}$, the boundaries for the two-phase region determined by Leroux, et al. ${ }^{1}$, are somewhat inaccurate. Based on our volume fraction analysis for the $\mathrm{Co}_{41.7} \mathrm{Pt}_{58.3}$ sample, applying the lever rule at $700{ }^{\circ} \mathrm{C}$ under the assumption that Leroux, et al.'s solvus bordering the $\mathrm{L}_{2}$ phase at $700{ }^{\circ} \mathrm{C}$ (Figure 3.1), which occurs at $61.6 \% \mathrm{Pt}$, is accurate, we find that the solvus bordering $\mathrm{L} 1_{0}$ should have a value of $57.5 \% \mathrm{Pt}$, compared to $58.8 \% \mathrm{Pt}$ as determined by Leroux et al. Thus, the two-phase field subtends about 4 atom $\%$ at $700{ }^{\circ} \mathrm{C}$, as opposed to about 3 atom $\%$ indicated in previously published studies. 
Although this investigation was conducted on samples with a Pt- rich composition relative to the equimolar alloy, the $\mathrm{A} 1 \rightarrow \mathrm{L}_{0}$ ordering transformation is similar to reports in the literature for both compositions. In situ annealing of CoPt single crystals in the TEM by Penisson, et al. ${ }^{35}$ was observed to lead to the formation of tweed contrast, which then evolved to form small, ramified plates of $\mathrm{L}_{0}$ on $\{110\}$ habit planes, and finally this develops into a coarse, fully transformed lamellar microstructure. While annealing at $700{ }^{\circ} \mathrm{C}$, our $\mathrm{Co}_{41.7} \mathrm{Pt}_{58.3}$ alloy attained the observed peak hardness in 2.5 hours. The coercivity was still very low after annealing for 15 minutes at that temperature. However, Hadjipanayis and Gaunt, who used TEM and XRD to correlate magnetic properties with microstructure in $\mathrm{Co}_{48} \mathrm{Pt}_{52}$ alloys ${ }^{45}$, observed that the peak hardness of 4000 Oe occurred after only a $17 \mathrm{~min}$ annealing treatment at $700{ }^{\circ} \mathrm{C}$, corresponding to microstructural length-scale of $20 \mathrm{~nm}$. Xiao, et al. ${ }^{46}$, obtained a peak coercivity of $5 \mathrm{kOe}$, again with microstructural evolution similar to what we observed.

Exchange-coupling behavior is suggested by the high remanence ratios observed in all the $\mathrm{Co}_{41.7} \mathrm{Pt}_{58.3}$ samples annealed at $600{ }^{\circ} \mathrm{C}$, (Figure 3.4). The peak hardness sample, aged at $600{ }^{\circ} \mathrm{C}$ for 96 hours which has a $15 \mathrm{~nm}$ period to the tweed contrast [Figure 3.9 (a,d)], also evidences the highest $\mathrm{M}_{\mathrm{r}} / \mathrm{M}_{\mathrm{s}}=0.80$. This declines only slightly, to 0.77 , upon extended annealing to 720 hours, when the $\mathrm{L}_{0}$ particle length-scale increases to $20-40 \mathrm{~nm}$. For aging at $700{ }^{\circ} \mathrm{C}$, exchangecoupling is rapidly lost as the system coarsens very rapidly from accelerated kinetics at this temperature. Exchange-coupling in nanoscale, partially-transformed CoPt samples has been previously reported by others. Hadjipanayis and Gaunt ${ }^{45}$ asserted that exchange coupling occurred between adjacent hard-magnetic $\mathrm{L1}_{0}$ particles in coherent twin orientations, although the sample had only partially transformed from the A1 matrix to the ordered $\mathrm{L} 1_{0}$ phase at the stage corresponding to the maximum remanence ratio. Xiao, et al. ${ }^{46}$, also reported remanence ratios as high as 0.78 in partially transformed films, which they associated with coupling between the hard magnetic $\mathrm{L} 1_{0}$ phase and the soft $\mathrm{A} 1$ phases. Using recoil analysis, they showed that recoverability (as a proxy for reversibility) varied as a function of annealing conditions, even when comparing samples with identical remanence ratios. Recoil analysis on our samples is currently underway. However, it is clear from the preliminary results as well as from the previous studies that interpretation of the exchange coupling behavior in partially transformed $\mathrm{L1}_{0}$ samples is challenging since a range of transformation length-scales occurs, and more 
importantly, a range of order parameters is present, indicating a distribution of magnetocrystalline anisotropy values.

The mechanisms for magnetization reversal in our peak coercivity sample (aged at $600^{\circ} \mathrm{C}$ for 96 hours) are quite interesting. As we have noted previously, TEM strain contrast indicates an array of nanoscale $\mathrm{L}_{0}$ transformation centers embedded in the A1 matrix, shown in Figure 3.9 (a,d) with a particle spacing of approximately $15 \mathrm{~nm}$, suggesting an even smaller particle diameter. From Brown's ${ }^{47}$ prediction for the lower bound on the critical single domain particle size $\left(2 \mathrm{r}_{\mathrm{c} 0}\right)$ for spherical particles in zero applied field, we have $2 r_{c 0}=2\left(3.61 / M_{s}\right)(A / 4 \pi)^{1 / 2}$, all quantities being in cgs units. From our data, $\mathrm{a} \approx 3.8 \mathrm{~A}$ and $\mathrm{M}_{\mathrm{s}}=511 \mathrm{emu} / \mathrm{cc}$; we have estimated the exchange constant to be $\mathrm{A}=\mathrm{k}_{\mathrm{B}} \mathrm{T}_{\mathrm{c}} / \mathrm{a}=2.5 \times 10^{-6} \mathrm{ergs} / \mathrm{cm}$. This yields a critical single domain particle size of $2 \mathrm{r}_{\mathrm{c} 0}=124 \mathrm{~nm}$. Thus, the $\mathrm{L}_{0}$ transformation centers in the $\mathrm{Co}_{41.7} \mathrm{Pt}_{58.3}$ sample are rigorously single domain under zero applied field. In this context, Xiao, et al. ${ }^{46}$, have applied the coherent rotation of magnetization reversal to explain the observed coercivity, using the anisotropy field, $\mathrm{H}_{\mathrm{a}}$, to estimate the coercivity as $\mathrm{H}_{\mathrm{a}}=2 \mathrm{~K} / \mathrm{M}_{\mathrm{s}}=60-120 \mathrm{kOe}$, requiring inputs for the values used for $\mathrm{K}$ and $\mathrm{M}_{\mathrm{s}}$. In order to explain their observed $\mathrm{H}_{\mathrm{c}} \approx 5 \mathrm{kOe}$, they had to employ unusually low values for the magnetocrystalline anisotropy. Thus, we can see that it is not properly justified to invoke the Stoner-Wohlfarth theory for coherent rotation. Furthermore, this theory assumes non-interacting nanoparticles ${ }^{48}$. In case of partially transformed $\mathrm{A} 1 \rightarrow \mathrm{L} 1_{0}$, the hard magnetic $\mathrm{L} 1_{0}$ nanoparticles are embedded in, and exchange coupled to a soft ferromagnetic A1 matrix having a larger saturation magnetization. Zhou, et al. ${ }^{49}$ have used micromagnetics to investigate magnetization reversal in magnetically hard nanoparticles in a soft ferromagnetic matrix as a function of exchange coupling strength. According to them, at low exchange coupling, individual particles undergo magnetization reversal by coherent rotation in a spatiallyindependent fashion, which they referred to as "nucleation" behavior. However, for sufficiently large exchange coupling, extended regions of particles with parallel moments are formed, which can be thought of as domains ${ }^{50}$. In that case, i.e., for high remanence ratios, their calculations indicate the formation of a classical domain wall, which is mostly in the soft matrix, and moves by "jumping" the hard nanoparticles. While the individual nanoparticles may still coherently rotate, they do so in a cooperative manner that reduces the effective anisotropy field. 
The coercivity can, in principle, be quite low relative to the intrinsic anisotropy field for samples exhibiting this behavior, which they refer to as "pinning" ${ }^{4}$. In a subsequent paper Zhou, et al. further published similar calculations for $\mathrm{FePt} \mathrm{L1}_{0}$ nanoparticles embedded in a semi-hard FeNiPt matrix ${ }^{51}$. They found similar behavior, where for an exchange constant $\mathrm{A}>1.5 \times 10^{-6}$ ergs/cm, the coercivity was seen to be dominated by 'pinning' of domain walls in the softer phase as they 'jumped' the hard-phase nanoparticles. While their predicted coercivities are higher $\left(18 \mathrm{kOe}\right.$ for $\left.\mathrm{A}=2.5 \times 10^{-6} \mathrm{ergs} / \mathrm{cm}\right)$ than observed here, the predicted value would clearly be reduced for $\mathrm{L}_{0}$ particles embedded in the magnetically softer matrix present in our case, on account of the particles having reduced $\mathrm{K}$ from incomplete ordering and non-stoichiometry.

The shape of the initial magnetization curve of our peak hardness $\mathrm{Co}_{41.7} \mathrm{Pt}_{58.3}$ sample is in accordance with domain wall pinning as the dominant mechanism for magnetization reversal. Although we do not employ directly-relevant micromagnetics calculation of the modified critical rotation field as discussed by Zhou et al. ${ }^{49}$, we are able to successfully apply a classical pinning model to estimate the expected coercivity associated with motion of a wall through the soft ferromagnetic matrix as it encounters partially-ordered non-stoichiometric $\mathrm{L}_{0}$ nanoparticles. A $180^{\circ}$ domain wall experiences a resistive force as it moves through a heterogeneous region under an applied magnetic field, from a phase with magnetocrystalline anisotropy constant $\mathrm{K}^{\prime}$, the FCC phase in our case, to one with anisotropy constant $\mathrm{K}$ corresponding to the $\mathrm{L} 1_{0}$ phase. The coercive field, $\mathrm{H}_{\mathrm{c}}$, is defined when the force exerted by the magnetic field per unit area of the domain wall exactly balances the resistive force per unit area of the wall. The magnetic force per unit area equals $2 \mathrm{H}_{\mathrm{c}} \mathrm{M}_{\mathrm{s}}$. Gaunt proposed a simple but descriptive model wherein the force resisting wall motion is due to the energy gradient of the domain wall, $\mathrm{d} \gamma / \mathrm{dx}, \gamma$ being the domain

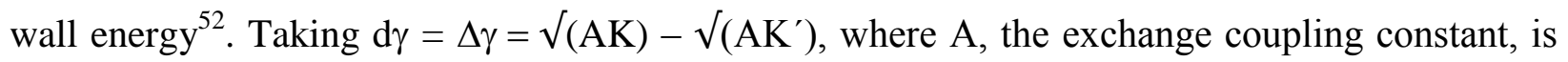
taken to be $2.5 \times 10^{-6} \mathrm{ergs} / \mathrm{cm}$ as discussed previously, and assumed to be equal in both phases since there is no significant variation in the Curie temperature of the two phases. $\Delta \mathrm{x}$ is taken to be $\lambda^{\prime}, \lambda^{\prime}$ being the characteristic microstructural lengthscale obtained from the TEM micrograph of this peak hardness $\mathrm{Co}_{41.7} \mathrm{Pt}_{58.3}$ sample (Figure 3.9 a). Gaunt's equation may then be written as: $2 \mathrm{H}_{\mathrm{c}} \mathrm{M}_{\mathrm{s}}=A\left(4 \sqrt{(\mathrm{AK})}-4 \sqrt{\left.\left(\mathrm{AK}^{\prime}\right)\right) /\left(\lambda^{\prime}\right)}\right.$ 
$A$ being the area of $\mathrm{L}_{0}$ pinning sites per unit area of domain wall, which can have a value ranging from $0-1$. Assuming that $K\left(\mathrm{L1}_{0}\right) \gg \mathrm{K}^{\prime}(\mathrm{A} 1)$, we have

$$
\mathrm{H}_{\mathrm{c}} \approx 2 A \sqrt{ }(\mathrm{AK}) /\left(\lambda^{\prime} \mathrm{M}_{\mathrm{s}}\right)
$$

From our data, we have $\lambda^{\prime} \approx 12 \mathrm{~nm}$ and $\mathrm{M}_{\mathrm{s}}=511 \mathrm{emu} / \mathrm{cc}$ in the $\mathrm{Co}_{41.7} \mathrm{Pt}_{58.3}$ sample exhibiting maximum coercivity. For the case of stoichiometric, fully-ordered $\mathrm{L}_{0} \mathrm{CoPt}, \mathrm{K}=5 \times 10^{7} \mathrm{erg} / \mathrm{cc}^{9}$. However, accounting for the Pt content in our samples and the facts that the $\mathrm{L} 1_{0}$ particles formed are not fully ordered, stoichiometric nor fully tetragonal at peak coercivity, we extrapolate the work of Shima, et al. ${ }^{53}$, to arrive at a relevant value for this analysis, $\mathrm{K}=1.5 \times 10^{7} \mathrm{erg} / \mathrm{cm}^{3}$. Substituting these values in Eq. 3.18, we can reproduce the peak hardness observed here, $\mathrm{H}_{\mathrm{c}}=$ 4000 Oe for $\mathcal{A}=0.2$, which is a plausible value (i.e., it is less than unity).

At first glance, it may not appear justified to use a simple continuum pinning model to predict the coercivity when the actual predominant mechanism is based on the domain wall jumping past nanoparticles. However, all domain wall pinning models can be thought of in terms of an energy landscape, where the wall, which is initially in a low energy configuration, must pass through the activation barrier presented by the pinning sites so as to reach the next equivalent low energy configuration. In the present continuum model, we have used the hard-phase wall energy in lieu of the activation barrier associated with the coherent rotation energy of the nanoparticles in their local exchange-coupled environment employed by Zhou, et al. ${ }^{49,51}$ In order for this to be reasonable, the wall thickness must be similar to the dimensions of the particle. The wall thickness is given by $\delta=4(\mathrm{~A} / \mathrm{K})^{1 / 2}$ and using the abovementioned values for $\mathrm{A}$ and $\mathrm{K}$ gives $\delta=$ $16 \mathrm{~nm}$, which is similar to the mean particle size of $15 \mathrm{~nm}$. A schematic representation of the domain wall advancement process shown in Figure 3.13 illustrates how the advancement of the domain wall might proceed by the motion of "kinks", in a two-dimensional analog of dislocation glide, as suggested by the work of Zhou, et al. ${ }^{49}$ As shown in the figure, the kink portion of the wall would have a Néel configuration of spins, whereas the planar wall portions would be in Bloch configuration. Advancement past an $\mathrm{L} 1_{0}$ nanoparticle to move the kink would happen via coherent rotation, but in our approximate treatment, the Néel and Bloch portions would pass through the $\mathrm{L}_{0}$ particle. 
The peak hardness material among samples annealed at $700{ }^{\circ} \mathrm{C}$, corresponding to an annealing time of 2.5 hours has a more sharply kinked initial magnetization curve, possibly arising from the Stoner-Wohlfarth rotation mechanism for non-interacting particles. However, in the absence of TEM analysis for this sample, we cannot conclusively articulate how the magnetization reversal mechanisms in this sample would differ from that described above.

There is a very intriguing evolution in magnetic properties with aging time and temperature, as shown in Figure 3.3. Continued aging at $600{ }^{\circ} \mathrm{C}$, beyond peak hardness corresponding to 96 hours annealing time, produces a subsequent decrease in coercivity, attributed to the coarsening of lengthscales, as indicated by Eq. 3.18. Prolonged annealing at this temperature gradually allows for a recovery of the hardness. For instance, the progress in transformation would lead to an increase in tetragonality, and hence $\mathrm{K}$, as well as an increase in pinning sites, $\mathcal{A}$. Although after an extended annealing time of 720 hours $\lambda$ is about twice that in the peak hardness sample, a net doubling of $A \sqrt{ } \mathrm{K}$, which is reasonable, would account for the coercivity of $\sim 4000$ Oe, in accordance with Eq. 3.18. However, it is quite possible that the model described by Figure 3.13 and Eq. 3.18 does not apply to the coarsened sample at 720 hours since the sample contains $72 \%$ by volume of the $\mathrm{L}_{0}$ phase in only $28 \%$ matrix. It is possible that by this stage the coercivity mechanism transitions from domain wall pinning by nanoparticles to domain wall pinning by anti phase boundaries. APBS are formed as $\mathrm{L1}_{0}$ particles grow to impingement. Magnetization reversal via domain wall pinning by APBs has been discussed for polytwinned $\mathrm{L} 1_{0}$ alloys in the literature. $^{22}$

It may be noted that a local maximum occurs in the data for the $\mathrm{M}_{\mathrm{s}}$ versus annealing plot at $600{ }^{\circ} \mathrm{C}$ [Figure 3.3(c)] at an annealing time of 168 hours annealing. Although this is perhaps unexpected, we think that the initial rapid decline in $\mathrm{M}_{\mathrm{s}}$ after 24 hours annealing at $600{ }^{\circ} \mathrm{C}$ results from the extensive inhomogeneous strain and local disorder concomitant to the formation of the $\mathrm{L}_{0}$ transformation centers. Further complexity is observed in the magnetic aging behavior exhibited at $700{ }^{\circ} \mathrm{C}$, where the enhanced diffusion kinetics facilitate and expedite the complete transformation to coarse $\mathrm{L}_{0}$ plates. A maximum in $\mathrm{H}_{\mathrm{c}}$ occurs at only 2.5 hours. A secondary maximum in $\mathrm{H}_{\mathrm{c}}$ is observed thereafter, at 24 hours annealing time, which possibly originates from a change in the dominant pinning mechanisms in the largely transformed sample containing $\mathrm{L1}_{0}$ plates. For instance, the magnetization reversal in case of this sample could be governed by 
domain wall pinning by the antiphase domain boundaries present in the $\mathrm{L} 1_{0}$ plates ${ }^{23}$. The $\mathrm{L} 1_{2}$ phase was seen to form following very long anneals as discussed previously based on the quantitative analysis of the x-ray data. The final increase in coercivity is observed at extended annealing times, and we attribute this to domain wall pinning by these $\mathrm{L}_{2}$ nanoparticles embedded in the $\mathrm{L} 1_{0}$ matrix.

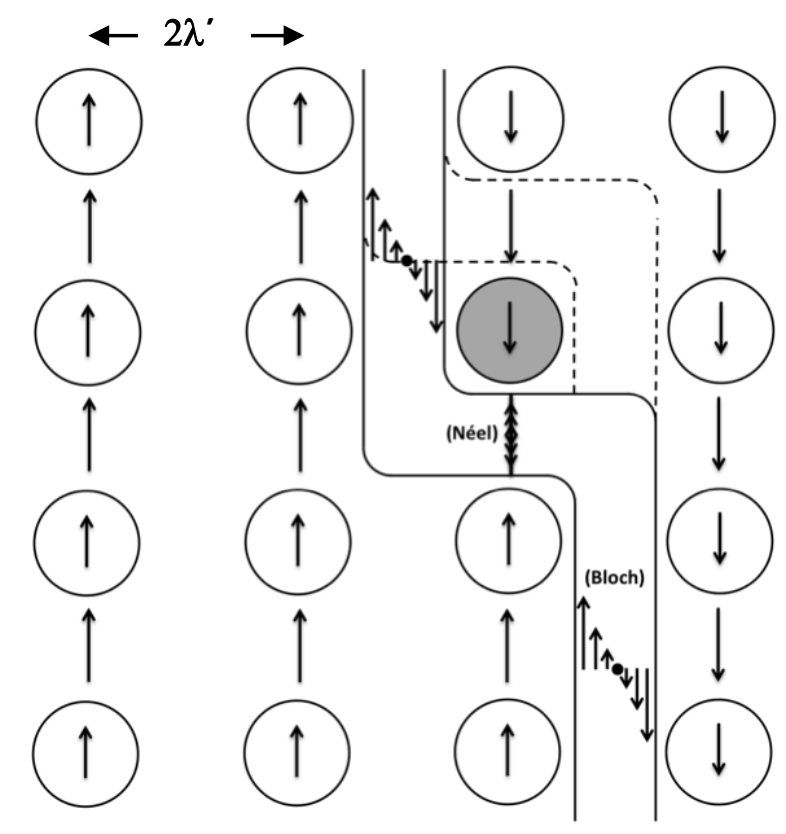

Figure 3.13: $\mathrm{L} 1_{0}$ nanoparticles (circles) embedded in a softer $\mathrm{A} 1$ matrix, showing a domain wall and its internal spin configurations. The domain wall advances by movement of the Néel kink to the new position shown with dashed lines, thus reversing the particle (and nearby matrix) shown in gray. 


\section{Summary}

Compositions in the vicinity of the Co-Pt eutectoid isotherm were investigated, with a focus on establishing processing-structure-property relationships, and elucidating the nature of the phase transformation. Upon annealing, $\mathrm{Co}_{41.7} \mathrm{Pt}_{58.3}$ underwent the disorder $\rightarrow$ order transformation, from FCC to predominantly non-stoichiometric $\mathrm{L} 1_{0}$. $\mathrm{L}_{2}$ formation was inferred to occur in the later stages of this transformation, redefining the published boundaries of the $\mathrm{L}_{0}+\mathrm{L}_{2}$ twophase region. The magnetic properties of this alloy varied intricately as a function of aging time and temperature, accompanying changes in the microstructural lengthscales, phase evolution, and dominant reversal mechanisms. The maximum room temperature coercivity of $4000 \mathrm{Oe}$, and remanence to saturation ratio of 0.8 , indicative of strong exchange coupling between the $\mathrm{L} 1_{0}$ and A1 matrix, were attained after annealing at $600^{\circ} \mathrm{C}$ for 96 hours. This peak hardness was obtained in the early stages of the disorder $\rightarrow$ order transformation, with an $\mathrm{L}_{0}$ particle size of $\sim 15 \mathrm{~nm}$. Domain wall pinning by $\mathrm{L}_{0}$ nanoparticles is proposed to control magnetization reversal in the peak coercivity sample. XRD and VSM indicated that the $\mathrm{Co}_{37.6} \mathrm{Pt}_{62.4}$ alloy ordered exclusively to the soft ferromagnetic $\mathrm{L}_{2}$ phase. 


\section{Compositions on the eutectoid isotherm: $\mathrm{Co}_{40.2} \mathrm{Pt}_{59.8}$ and $\mathrm{Co}_{38.8} \mathrm{Pt}_{61.2}$}

The unique nanochessboard structure, a quasi-periodic two-dimensional tiling of $\mathrm{L}_{0}$ and $\mathrm{L}_{2}$ nanorods resulting from self assembly via eutectoid decomposition, was described in Chapter 1. We have obtained this chessboard microstructure following appropriate thermal processing of alloys of specific compositions. Alloy compositions lying on the eutectoid isotherm, $\mathrm{Co}_{40.2} \mathrm{Pt}_{59.8}$ and $\mathrm{Co}_{38.8} \mathrm{Pt}_{61.2}$, were selected for the study in order to ensure accessing the $\mathrm{L}_{0}+\mathrm{L} 1_{2}$ two-phase regime and the desired microstructure. These compositions are indicated in Figure 2.1. The formation of the nanochessboard necessitates the proper development of the template for the chessboard structure, i.e., the periodic composition fluctuations associated with the pseudospinodal decomposition process. Continuous cooling from 750 to $600{ }^{\circ} \mathrm{C} @ 40{ }^{\circ} \mathrm{C} /$ day fulfills this requirement and readily produced a chessboard microstructure.

A summary of the samples processed via continuous cooling is presented in Figure 4.1. As illustrated in the figure, samples were continuously cooled from $750{ }^{\circ} \mathrm{C}$ to $690{ }^{\circ} \mathrm{C}, 650{ }^{\circ} \mathrm{C}$ and $600{ }^{\circ} \mathrm{C}$. Although a chessboard structure has been observed in Co-Pt by Leroux et al. ${ }^{1}$ and Le Bouar et al. ${ }^{2}$, we are the first to determine the associated magnetic properties and explore the underlying structure-property relationship. The variation in magnetism data with thermal processing variables and composition is a valuable tool in understanding the underlying microstructure and phases present, as well as the magnetization reversal mechanism. 

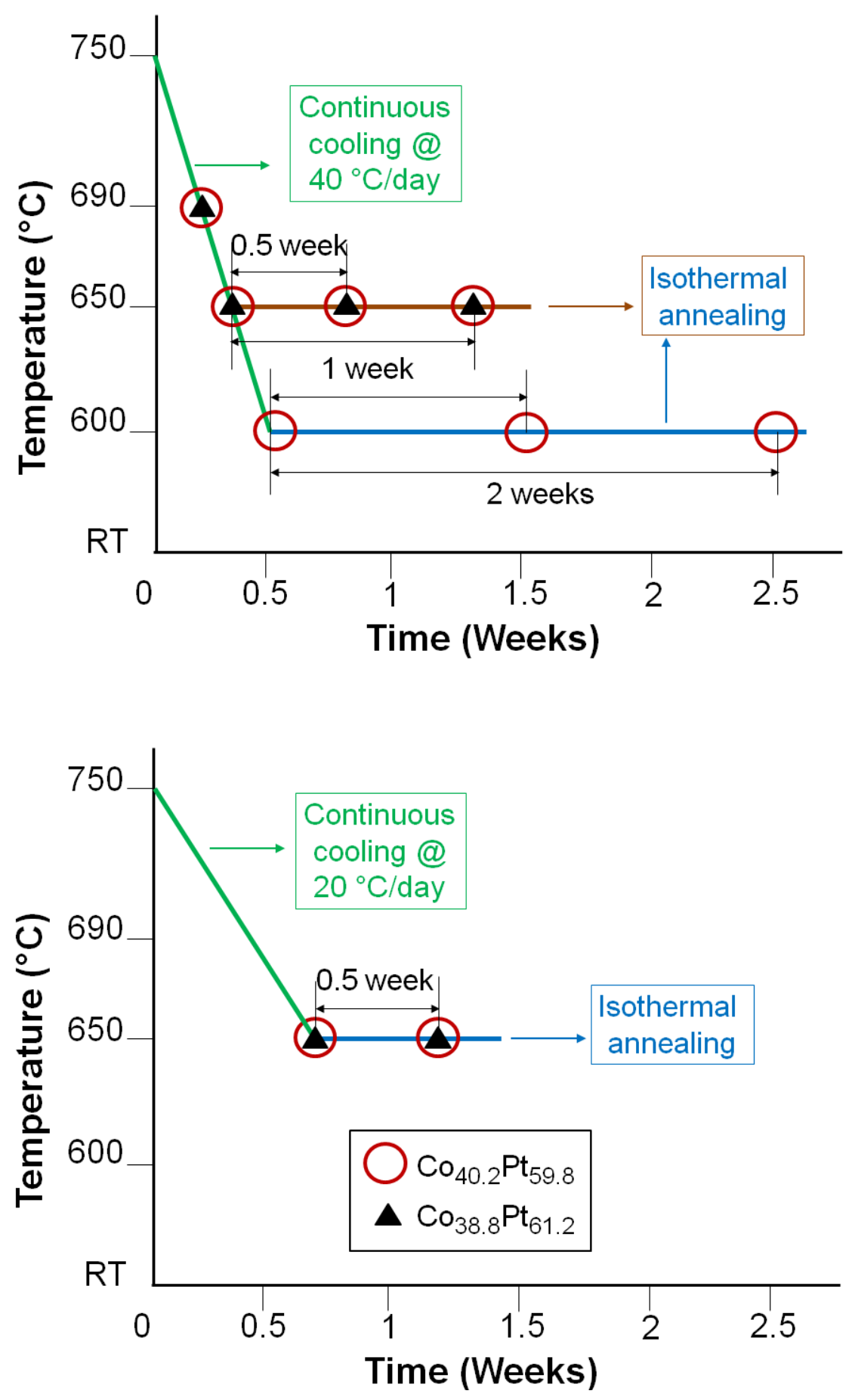

Figure 4.1: The processing space for continuously cooled samples from $\mathrm{Co}_{40.2} \mathrm{Pt}_{59.8}$ and $\mathrm{Co}_{38.8} \mathrm{Pt}_{61.2}$ alloys. 


\section{$\underline{\text { A. Results and Discussion }}$}

\subsection{Progress of phase transformation: XRD}

A comparison of the high resolution XRD patterns obtained from the continuously cooled $\mathrm{Co}_{40.2} \mathrm{Pt}_{59.8}$ samples annealed for 0,1 and 2 weeks at $600{ }^{\circ} \mathrm{C}$ is presented in Figure 4.2. All three samples evidenced the nanochessboard microstructure in the TEM, as will be shown in subsection 4.1.3. An analysis of this data with respect to order parameters and volume fractions of the various phases gives valuable insights into the progress of the phase transformation with annealing time.
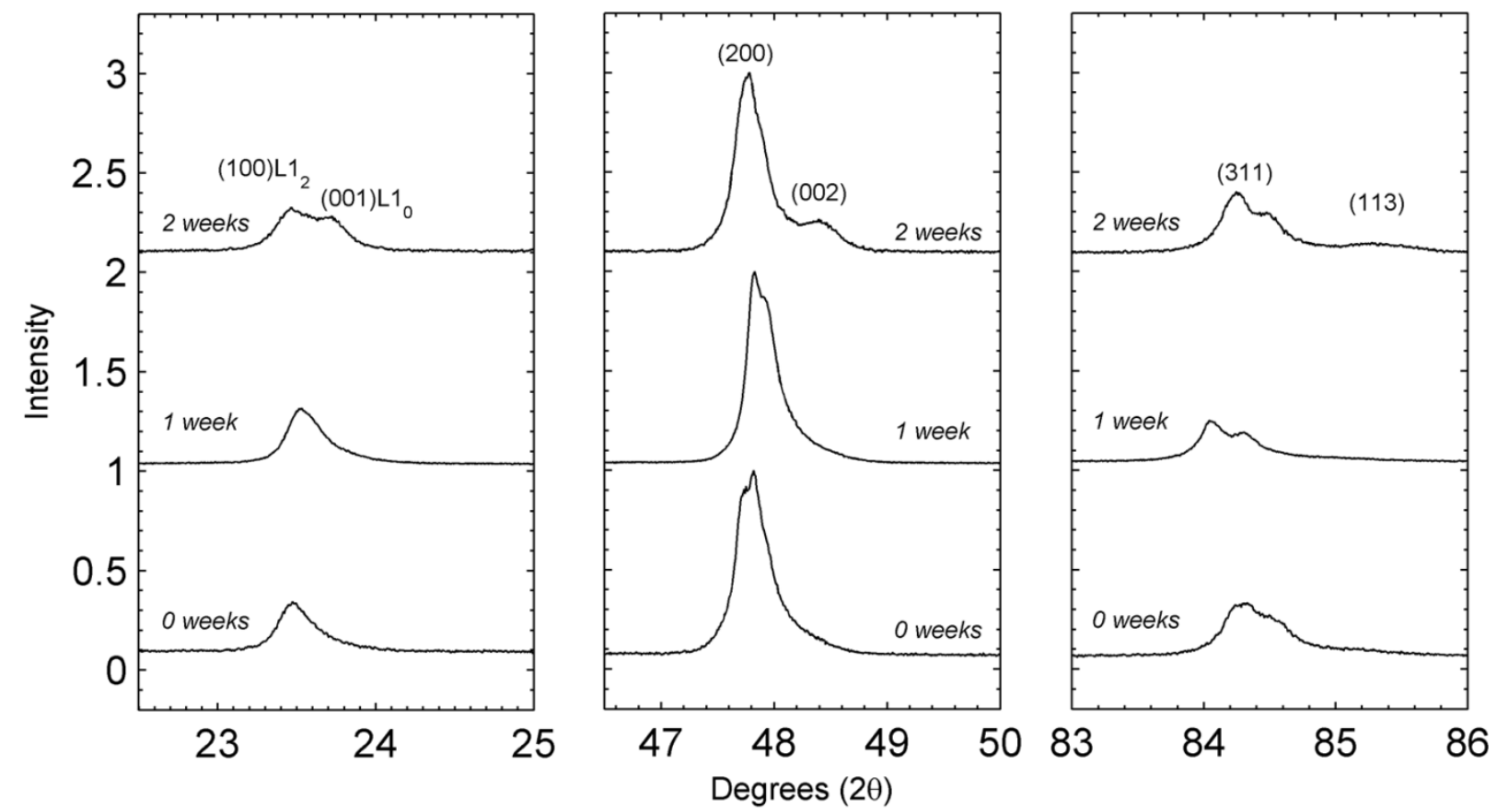

Figure 4.2: $\{001\},\{200\} /(002),\{311\} /(113) \mathrm{XRD}$ peaks from $\mathrm{Co}_{40.2} \mathrm{Pt}_{59.8}$ samples continuously cooled from 750 to $600{ }^{\circ} \mathrm{C} @ 40{ }^{\circ} \mathrm{C} /$ day, as a function of annealing time at $600{ }^{\circ} \mathrm{C}$.

Figure 4.2 focuses on the evolution of the $\{001\},\{200\} /\{002\},\{311\} /\{113\}$ peaks. The presence of an $\{001\}$ superlattice reflection clearly indicates the presence of an ordered phase. For the samples under consideration, this peak should contain contributions from the (001) $\mathrm{L} 1_{0}$ and the $\{100\} \mathrm{L}_{2}$ phases, which manifest as peak asymmetry or even peak splitting. The peak occurring at the lower (higher) value of $2 \theta$ corresponds to the $\mathrm{L1}_{2}\left(\mathrm{L1}_{0}\right)$ phase, as discussed in 
Chapter 3. We see that the (001) peak is asymmetric in case of the samples annealed for 0 and 1 week. The (001) $\mathrm{L1}_{0}$ and the $\{100\} \mathrm{L}_{2}$ peaks progressively separate out as the phase transformation progresses with increasing annealing time to result in distinct peak splitting in the 'overaged' sample produced after an annealing time of 2 weeks. The $\mathrm{L}_{0}$ phase must be present to enable forming the tweed modulation via the tetragonal transformation strain, which then develops into the chessboard microstructure. Hence, on the basis of the chessboard microstructure of the alloys under discussion, we believe that the separation of the (001) superlattice peaks with phase transformation corresponds to the formation and evolution of the $\mathrm{L1}_{2}$ phase.

A split $\{200\} /(002)$ and $\{311\} /$ (113) fundamental peak is an indicator of the tetragonality of the ordered $\mathrm{L} 1_{0}$ phase. This peak splitting is present at 0 and 1 weeks, since we require three peaks, corresponding to $\left\{200 \mathrm{~K}_{1}\right\},\left\{200 \mathrm{~K} \alpha_{2}\right\}$ and (002), to obtain a good fit to the XRD reflection. However, this splitting is more readily apparent in the sample annealed for 2 weeks. This further indicates that the tetragonality of the $\mathrm{L} 1_{0}$ phase increases with annealing time and that the strain evolves with the lengthscale.

As discussed in Chapter 3, the order parameter ' $\eta$ ' of the $L 1_{0}$ phase may be estimated from the intensities of the (001) and (002) peaks associated with this phase. From $\boldsymbol{\eta}=\mathbf{0 . 9} * \sqrt{ }\left[\mathbf{I}_{\mathbf{0 0 1}} / \mathbf{I}_{\mathbf{0 0 2}}\right]$, we obtain order parameters of $0.44,0.86$ and 0.78 for annealing periods of 0,1 and 2 weeks, respectively. Based on the phase diagram which suggests an equilibrium $\mathrm{L} 1_{0}$ composition of $\mathrm{Co}_{58.4} \mathrm{Pt}_{41.6}$ at $600{ }^{\circ} \mathrm{C}$, the maximum attainable order parameter would be $2 *(1-0.416)$ or 0.83 . That the intermediate annealing period appears to yield an unfeasible order parameter may indicate the limits of our analysis, since we have to fit overlapping, relatively weak Bragg peaks in order to perform this analysis. The data does suggest that the $\mathrm{L} 1_{0}$ phase becomes progressively more ordered with annealing time, until the order parameter attains its maximum achievable value.

The transformed material contains the soft retained A1 and/or ordered $\mathrm{L1}_{2}$ phases and the hard $\mathrm{L1}_{0}$ phase. Thus, the total volume of material, $\mathrm{V}_{\text {tot, }}$ may be expressed as the sum of the volumes of the soft and hard phases, $\mathrm{V}_{\text {soft }}+\mathrm{V}_{\text {hard }}$. The relative volume fractions of the soft and hard phases, $\mathrm{V}_{\text {soft }} / \mathrm{V}_{\text {hard }}$, may be estimated as follows: 


$$
\mathrm{V}_{\text {tot }}=\mathrm{V}_{\text {soft }}+\mathrm{V}_{\text {hard }}=\left(\mathrm{V}_{\mathrm{A} 1}+\mathrm{V}_{\mathrm{L} 12}\right)+\mathrm{V}_{\mathrm{L} 10}=100 \text {, where } \mathrm{V}_{\text {soft }}=\left(\mathrm{V}_{\mathrm{A} 1}+\mathrm{V}_{\mathrm{L} 12}\right) \text { and } \mathrm{V}_{\text {hard }}=\mathrm{V}_{\mathrm{L} 10} \text {. }
$$

From Eq. 3.12, we can use the $\{001\}$ peak intensities to estimate the ratio $\mathrm{V}_{\mathrm{L} 12} / \mathrm{V}_{\mathrm{L} 10}$ :

$$
\mathrm{V}_{\mathrm{L} 12} / \mathrm{V}_{\mathrm{L} 10}=4 /\left[3\left(\mathrm{I}_{001}\right)_{\mathrm{L} 10} /\left\{\mathrm{I}_{100}\right\}_{\mathrm{L} 12}\right] \text {. }
$$

We may obtain the ratio of the soft and hard phases with using the $\{311\}$ and (113) split peaks.

$$
\left[\left(\mathrm{I}_{311}+\mathrm{I}_{131}\right)_{\text {hard }} /\left(\mathrm{I}_{311}+\mathrm{I}_{131}\right)_{\text {soft }}\right]=\mathrm{F}_{\text {hard }}^{2} \cdot \mathrm{m}_{\text {hard }} \cdot \mathrm{LP}_{\text {hard }} /\left(\mathrm{F}_{\text {soft }}^{2} \cdot \mathrm{m}_{\text {soft }} \cdot \mathrm{LP}_{\text {soft }}\right) \ldots \text { (Eq. 4.1) }
$$

As discussed in Chapter 3, the structure factors for these non-stoichiometric phases are identical for both the hard and soft phases: $4\left(\mathrm{X}_{\mathrm{A}} \mathrm{f}_{\mathrm{A}}+\mathrm{X}_{\mathrm{B}} \mathrm{f}_{\mathrm{B}}\right)$ for the $\mathrm{L} 1_{0}$ and $\mathrm{L1}_{2}$ phases and $4 \mathrm{f}_{\mathrm{av}}=$ $4\left(\mathrm{X}_{\mathrm{A}} \mathrm{f}_{\mathrm{A}}+\mathrm{X}_{\mathrm{B}} \mathrm{f}_{\mathrm{B}}\right)$ for $\mathrm{A} 1$; the Lorentz-polarization factors are similar as well. The multiplicity of the $\{311\}$ reflection is 6 for the $\mathrm{L}_{2}$ and $\mathrm{A} 1$ phases, and 4 for the hard $\mathrm{L} 1_{0}$ phase.

$$
\begin{gathered}
4\left(\mathrm{X}_{\mathrm{A}} \mathrm{f}_{\mathrm{A}}+\mathrm{X}_{\mathrm{B}} \mathrm{f}_{\mathrm{B}}\right), 4 \mathrm{f} \text { av } \\
\left\{\left(\boldsymbol{I}_{311}+\boldsymbol{I}_{131}\right)_{\text {hard }} /\left(\boldsymbol{I}_{311}+\boldsymbol{I}_{131}\right) \boldsymbol{L}_{\text {soft }}\right\}=4 / 6=2 / 3 \ldots(\text { Eq. } 4.2)
\end{gathered}
$$

If volume $\mathrm{V}$ of the hard phase corresponds to an intensity $\left(\mathrm{I}_{311}+\mathrm{I}_{131}\right)_{\text {hard }}=2 \mathrm{x}$, then, from Eq.4.2, an equal volume $\mathrm{V}$ of the soft phase corresponds to an intensity $\left(\mathrm{I}_{311}+\mathrm{I}_{131}\right)_{\text {soft }}=3 \mathrm{x}$.

Hence, a given volume $\mathrm{V}^{\prime}$ of the soft phase corresponds to an intensity $\left(\mathrm{I}_{311}+\mathrm{I}_{131}\right)_{\text {soft }}=3 \mathrm{x} * \mathrm{~V}^{\prime} /$ V. Thus, the ratio of observed intensities may be rewritten in terms of the volumes of the phases as:

$$
\left(\mathrm{I}_{311}+\mathrm{I}_{131}\right)_{\text {hard }} /\left(\mathrm{I}_{311}+\mathrm{I}_{131}\right)_{\mathrm{soft}}=2 /\left[3\left(\mathrm{~V}^{\prime} / \mathrm{V}\right)\right]
$$

Thus,

$$
\mathrm{V}_{\text {soft }} / \mathrm{V}_{\text {hard }}=2 /\left[3\left(\mathrm{I}_{311}+\mathrm{I}_{131}\right)_{\text {hard }} /\left(\mathrm{I}_{311}+\mathrm{I}_{131}\right)_{\text {soft }}\right]
$$

An observed intensity ratio $\left(\mathrm{I}_{311}+\mathrm{I}_{131}\right)_{\text {total }} / \mathrm{I}_{113}=\mathrm{X}$

$$
\Rightarrow\left[\left(\mathrm{I}_{311}+\mathrm{I}_{131}\right)_{\text {hard }}+\left(\mathrm{I}_{311}+\mathrm{I}_{131}\right)_{\text {soft }}\right] / \mathrm{I}_{113}=\mathrm{X}
$$

For the completely ordered $\mathrm{L} 1_{0}$ phase,

$$
\left(\mathrm{I}_{311}+\mathrm{I}_{131}\right)_{\text {hard }}=2 * \mathrm{I}_{113}
$$


From Eq. 4.4 and Eq. 4.5, we get:

$$
\left.\left(\mathrm{I}_{311}+\mathrm{I}_{131}\right)_{\mathrm{soft}}=(\mathrm{X}-2)^{*} \mathrm{I}_{113} \quad \text {...(Eq. } 4.6\right)
$$

Substituting Eq. 4.5 and Eq. 4.6 in Eq. 4.3,

$$
\mathrm{V}_{\text {soft }} / \mathrm{V}_{\text {hard }}=2 *(\mathrm{X}-2) /(3 * 2)
$$

We have estimated the relative volumes of the $\mathrm{L} 1_{0} / \mathrm{L} 1_{2}$ phases from an analysis of the split $\{001\}$ peaks and the relative volumes of the soft phases/ hard phase by analyzing the split $\{311\} /(113)$ peaks for the continuously cooled $\mathrm{Co}_{40.2} \mathrm{Pt}_{59.8}$ samples annealed for 0,1 and 2 weeks at $600{ }^{\circ} \mathrm{C}$. These results are presented below.

\section{Annealing time $=0$ weeks:}

From $\{001\}$ peak analysis, this sample contains $65 \% \mathrm{~L}_{2}$ and $35 \% \mathrm{L1}_{0}$, by volume.

From $\{311\} /$ (113) peak analysis, this sample contains $76 \%$ of the soft phase and $24 \%$ of the hard phase, by volume.

$\Rightarrow$ From $\{311\} /(113)$, let $V_{\text {soft }}=76, V_{\text {hard }}=24$

Then, $\{001\}: \mathrm{V}_{\mathrm{L} 12}=(65 / 35) * 24=44$

$\Rightarrow \mathrm{V}_{\mathrm{A} 1}=76-44=32$

\section{Annealing time = 1week:}

From $\{001\}$ peak analysis, this sample contains $50 \% \mathrm{~L}_{2}$ and $50 \% \mathrm{~L}_{0}$, by volume.

From $\{311\} /$ (113) peak analysis, this sample contains $64 \%$ of the soft phase and $36 \%$ of the hard phase, by volume.

$\Rightarrow$ From $\{311\} /(113)$, let $V_{\text {soft }}=64, V_{\text {hard }}=36$

Then, $\{001\}: \mathrm{V}_{\mathrm{L} 12}=(50 / 50) * 36=36$

$\Rightarrow \mathrm{V}_{\mathrm{A} 1}=64-36=28$ 


\section{Annealing time $=2$ weeks:}

From $\{001\}$ peak analysis, this sample contains $80 \% \mathrm{~L}_{2}$ and $20 \% \mathrm{~L}_{0}$, by volume.

From $\{311\} /$ (113) peak analysis, this sample contains $76.5 \%$ of the soft phase and $23.5 \%$ of the hard phase, by volume.

Given the uncertainties in the peak fit for (001), it is reasonable to take the ratio thus obtained to be the same as that obtained from the $\{311\} /(113)$ analysis. Assuming that the two numbers are almost equal could imply that we have no residual A1 at this stage, and that the sample contains $\approx 78 \%$ of the $\mathrm{L}_{2}$ phase and $22 \%$ of the $\mathrm{L}_{2}$ phase, by volume.

A summary of the results from this analysis is presented below in Table 4.1. The accuracy of volume fractions is estimated to be \pm 4 volume percent for a reasonable visual fit to the xrd background noise.

$\mathbf{L 1}_{\mathbf{0}}$

0 weeks

$21 \%$

1 week

2 weeks
$37 \%$

$22 \%$
$40 \%$

$40 \%$

$78 \%$

\section{A1}

$39 \%$

$23 \%$

$0 \%$

Table 4.1: Volume fractions of constituent phases in $\mathrm{Co}_{40.2} \mathrm{Pt}_{59.8}$ samples continuously cooled from 750 to $600{ }^{\circ} \mathrm{C} @ 40{ }^{\circ} \mathrm{C} /$ day, as a function of annealing time at $600{ }^{\circ} \mathrm{C}$.

From this data, it appears that even with only continuous cooling followed by water quenching, i.e., without isothermal annealing following the continuous cooling, the phase transformation is already at stage (d) in Figure 1.23 in that the $\mathrm{FCC} \rightarrow \mathrm{L}_{2}$ decomposition is already underway. We observe that the residual disordered A1 phase is continuously consumed with progressive isothermal annealing, and is completely consumed by 2 weeks. The variation in $\mathrm{L} 1_{0}$ fraction in 
the samples annealed for 1 and 2 weeks might suggest that small compositional inhomogeneities may exist in the $\mathrm{Co}_{40.2} \mathrm{Pt}_{59.8}$ alloy. If, for example, the 1 week sample had a slightly higher Co content than the 2 week sample, higher by about 0.7 at $\%$, then it would have an equilibrium volume fraction of $37 \% \mathrm{L1}_{0}$, as compared to $22 \% \mathrm{L1}_{0}$ in the $\mathrm{Co}_{40.2} \mathrm{Pt}_{59.8}$ sample at equilibrium, according to the lever rule at $600{ }^{\circ} \mathrm{C}$.

A comparison of the XRD patterns from $\mathrm{Co}_{40.2} \mathrm{Pt}_{59.8}$ and $\mathrm{Co}_{38.8} \mathrm{Pt}_{61.2}$ samples continuously cooled from 750 to $650{ }^{\circ} \mathrm{C} @ 40{ }^{\circ} \mathrm{C} /$ day and then water quenched reveals similar trends in both alloys, as shown in Figure 4.3. A superlattice (001) reflection is detected in both cases, confirming the presence of an ordered phase. However, we do not see any significant evidence for asymmetry or splitting of the (001) reflection for either composition, indicating that we have only one ordered phase present at this stage. As will be shown in subsection 4.1.4, a chessboard microstructure is present in both these alloys - which necessitates the presence of the $\mathrm{L} 1_{0}$ phase, since the transformation strain drives the spatial self-assembly. The $\{200\}$ and $\{311\}$ peaks do not appear appreciably split. Thus, we believe that an $\mathrm{L}_{0}$ phase with a low degree of tetragonal distortion and a disordered A1 phase are present at this stage of the phase transformation. From the lever rule, one would expect a greater volume fraction of the $\mathrm{L1}_{0}$ phase in the more Co-rich alloy, i.e., in $\mathrm{Co}_{40.2} \mathrm{Pt}_{59.8}$, and this is confirmed here based on the relatively higher intensity of the superlattice (001) reflection. 

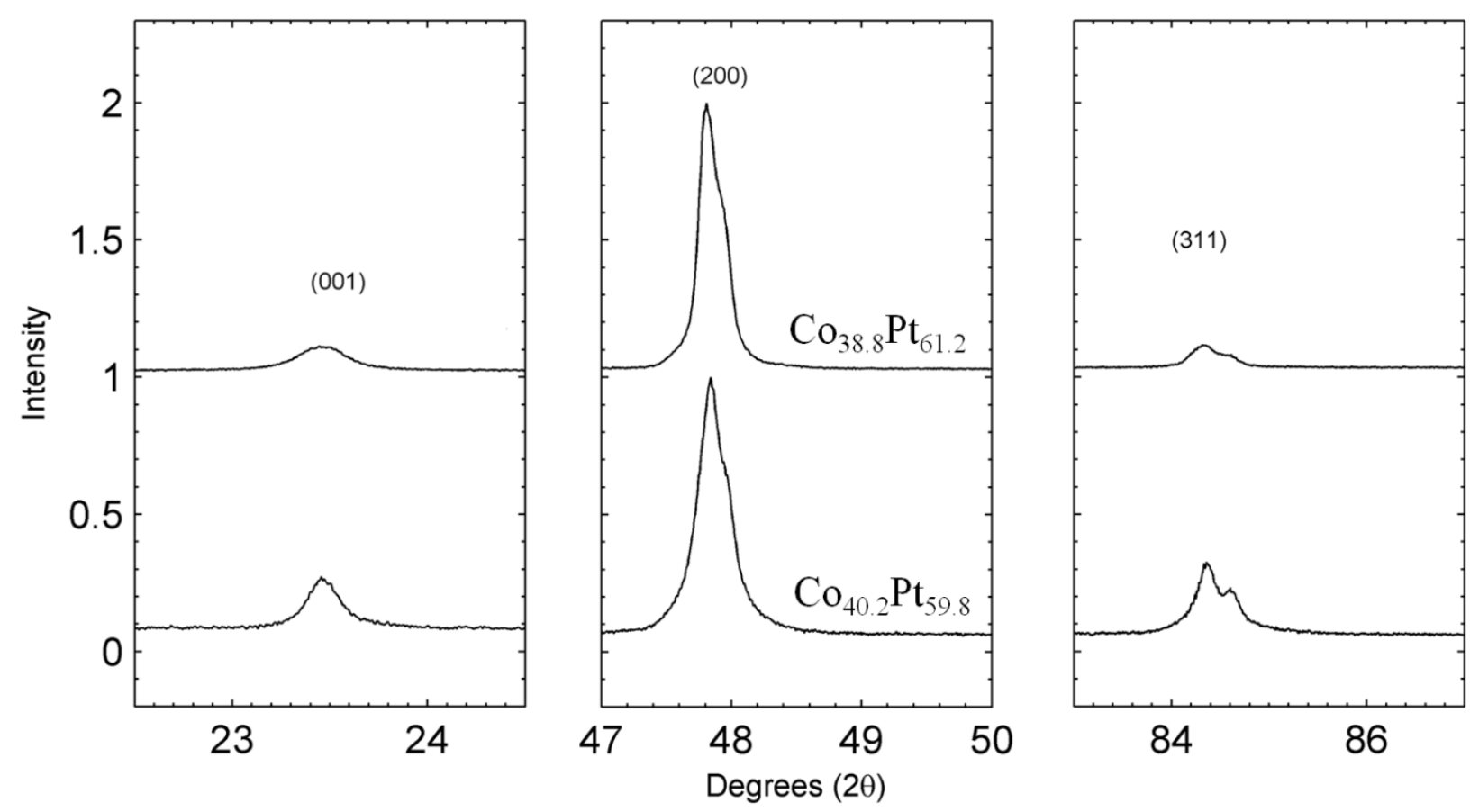

Figure 4.3: $\{001\},\{200\} /(002),\{311\} /(113)$ XRD peaks from $\mathrm{Co}_{40.2} \mathrm{Pt}_{59.8}$ and $\mathrm{Co}_{38.8} \mathrm{Pt}_{61.2}$ samples continuously cooled from 750 to $650{ }^{\circ} \mathrm{C} @ 40{ }^{\circ} \mathrm{C} /$ day and then water quenched.

\subsection{Accuracy of phase diagram: XRD}

Based on the XRD data for the $\mathrm{Co}_{40.2} \mathrm{Pt}_{59.8}$ chessboard sample continuously cooled from 750 to $600{ }^{\circ} \mathrm{C} @ 40{ }^{\circ} \mathrm{C} /$ day and then annealed for 2 weeks, and applying the lever rule, we have:

Mole fraction of $\mathrm{L}_{12}=\left(59.8-\mathrm{C}_{\mathrm{L} 10}\right) /\left(\mathrm{C}_{\mathrm{L} 12}-\mathrm{C}_{\mathrm{L} 10}\right) \approx$ Volume fraction of $\mathrm{L}_{2}$ (since density of the two phases may be taken to be similar); where $\mathrm{C}_{\mathrm{L} 10}$ and $\mathrm{C}_{\mathrm{L} 12}$ refer to the composition of the $\mathrm{L1}_{0}$ and $\mathrm{L1}_{2}$ phases respectively, with respect to Pt content.

$$
\Rightarrow\left(59.8-\mathrm{C}_{\mathrm{L} 10}\right) /\left(\mathrm{C}_{\mathrm{L} 12}-\mathrm{C}_{\mathrm{L} 10}\right) \approx 0.78
$$

This may be re-written as:

$$
0.22 \mathrm{C}_{\mathrm{L} 10}+0.78 \mathrm{C}_{\mathrm{L} 12} \approx 59.8
$$

From the previously published phase diagram ${ }^{1}$, the solvi bordering the $\mathrm{L}_{0}$ and $\mathrm{L}_{2}$ phases at 600 ${ }^{\circ} \mathrm{C}$ occur at 58.3 at.\% Pt and 62.9 at.\% Pt respectively. Thus, $\mathrm{C}_{\mathrm{L} 12}-\mathrm{C}_{\mathrm{L} 10}=4.6$ at.\% $\mathrm{Pt}$ at $600{ }^{\circ} \mathrm{C}$. If we assume the published value of $\mathrm{C}_{\mathrm{L} 12}=62.9$ at.\% $\mathrm{Pt}$ to be correct and substitute this in Eq. 
4.8, we get $\mathrm{C}_{\mathrm{L} 10}=48.8$ at.\% $\mathrm{Pt}$, implying a $\mathrm{C}_{\mathrm{L} 12}-\mathrm{C}_{\mathrm{L} 10}$ of 14.1 at.\% $\mathrm{Pt}$, far greater than the published value, which seems unreasonable. On the other hand, assuming the published value of $\mathrm{C}_{\mathrm{L} 10}=58.3$ at.\% Pt to be correct and substituting in Eq. 4.8, we get $\mathrm{C}_{\mathrm{L} 12}=60.2$ at.\% Pt, and implies that $\mathrm{C}_{\mathrm{L} 12}-\mathrm{C}_{\mathrm{L} 10}$ of 1.9 at.\% $\mathrm{Pt}$, which is smaller than the published value and also than the width of the eutectoid isotherm, and again seems unreasonable.

A third approach is based on the analysis of XRD data in Chapter 3, allowing us to instead estimate what the system composition would have to be, given the solvus compositions. The composition at the solvus bordering the $\mathrm{L}_{2}$ phase at $700{ }^{\circ} \mathrm{C}$ published in the phase diagram ${ }^{1}$, 61.6 at.\% $\mathrm{Pt}$, is assumed here to be correct since our Pt-rich $\mathrm{Co}_{37.6} \mathrm{Pt}_{62.4}$ alloy exclusively contained the $\mathrm{L1}_{2}$ phase after extended annealing at $700{ }^{\circ} \mathrm{C}$. Furthermore, the $\mathrm{Co}_{41.7} \mathrm{Pt}_{58.3}$ alloy contained $81 \% \mathrm{~L}_{0}$ and $19 \% \mathrm{L1}_{2}$ after extended annealing at $700{ }^{\circ} \mathrm{C}$. From the lever rule, the solvus bordering the $\mathrm{L}_{0}$ phase at $700{ }^{\circ} \mathrm{C}$ was estimated to be at 57.5 at. \% Pt. Based on the calculations at $700{ }^{\circ} \mathrm{C}$, the reported composition at the $\mathrm{L} 1_{0}$ solvus at $600{ }^{\circ} \mathrm{C}$ is adjusted and estimated to be 57 at. \% Pt. For the volume fractions estimated for the $\mathrm{Co}_{40.2} \mathrm{Pt}_{59.8}$ alloy from XRD analysis: $78 \% \mathrm{L1}_{2}, 22 \% \mathrm{~L}_{0}$, the lever rule predicts a system composition of $\mathrm{Co}_{38.3} \mathrm{Pt}_{61.7}$, i.e., 2 at\% greater than the average composition of $\mathrm{Co}_{40.2} \mathrm{Pt}_{59.8}$ indicated by ICP.

The disagreement with ICP is concerning. We showed with repeated trials that the error in precision of ICP is quite small, $<0.2$ at. \%. We do not know the accuracy of ICP associated with systematic errors, but calibration standards are applied to these analyses. These results then suggest that our samples may be compositionally inhomogeneous on a scale of hundreds of microns. A suggestion of this was already seen in the discussion of Table 4.1, where the $\mathrm{L}_{0}$ volume fraction differed between the samples annealed for 1 week and 2 weeks. Below, additional evidence for compositional inhomogeneity from TEM is presented.

\subsection{Evolution of microstructure: TEM}

The nanochessboard is not a true equilibrium structure but is metastable and hence, transformation kinetics are very important in influencing its formation or persistence during the

course of the heat treatment. ${ }^{28}$ The formation of the chessboard structure was verified and the effects of thermal processing parameters on microstructure were investigated using dark field 
(DF) TEM. TEM is the only technique to detect the presence or absence of an underlying chessboard microstructure; however it does not allow the location of residual A1 to be determined.

A chessboard structure was obtained in $\mathrm{Co}_{40.2} \mathrm{Pt}_{59.8}$ and $\mathrm{Co}_{38.8} \mathrm{Pt}_{61.2}$ samples upon gradual cooling to from 750 to $650{ }^{\circ} \mathrm{C} @ 40{ }^{\circ} \mathrm{C} /$ day followed by water quenching, as shown in Figure 4.4 (a) and (b) respectively. Chessboard colonies are formed along all three cube-axis directions of any given grain; i.e., the rod axis of chessboard colonies may lie along any of the three cube directions. The observation of colonies of side-on chessboards, i.e., with rod axes aligned orthogonal to the rod axes of the end-on chessboards, in addition to the familiar end-on chessboards confirmed this, as shown in Figure 4.4 (a). Side-on chessboards are indicated by green arrows here. SADPs of the two samples, from the regions imaged in Figs. 4.4 (a) and (b) can be seen in Figs. 4.4 (c) and (d) respectively. The presence of a chessboard structure containing the $\mathrm{L}_{2}$ phase and two orientation variants of the $\mathrm{L} 1_{0}$ phase is consistent with the dark field images and electron diffraction patterns.

Continuous cooling from 750 to $600{ }^{\circ} \mathrm{C} @ 40{ }^{\circ} \mathrm{C} /$ day followed by isothermal annealing at 600 ${ }^{\circ} \mathrm{C}$ also produced the nanochessboard structure in $\mathrm{Co}_{40.2} \mathrm{Pt}_{59.8}$ samples. Figure 4.5 shows an overview of $110 \mathrm{DF}$ images of the chessboard structure seen in samples continuously cooled from 750 to $600{ }^{\circ} \mathrm{C} @ 40{ }^{\circ} \mathrm{C} /$ day and then annealed for various durations - 0,1 and 2 weeks at $600{ }^{\circ} \mathrm{C}$ (a). In addition to end-on chessboard colonies, colonies of side-on chessboards were also seen in the sample annealed for 0 weeks and are indicated by green arrows. Thus, here as well, chessboard colonies formed along all three cube-axis directions.

Additional images corresponding to these samples annealed for 0,1 and 2 weeks are shown in Figure 4.6 (a), 4.7 (a) and 4.8 (a) respectively. Figure 4.6 (a) provides a magnified view of the chessboard observed on the lower right hand corner of the ' 0 week' sample in Figure 4.5. Figure 4.7 (a) shows a 110 DF image of a well-developed chessboard from another region in the '1 week' sample. Figure 4.8 (a) corresponds to another area in the '2 week' sample. SADPs from the regions imaged in Figures 4.6 (a), 4.7 (a) and 4.8 (a) are included as Figures 4.6 (b), 4.7 (b) and 4.8 (b) respectively. Together, dark field imaging and electron diffraction patterns both confirm the presence of the chessboard structure containing $\mathrm{L}_{2}$ and two orientation variants of the $\mathrm{L} 1_{0}$ phase. 


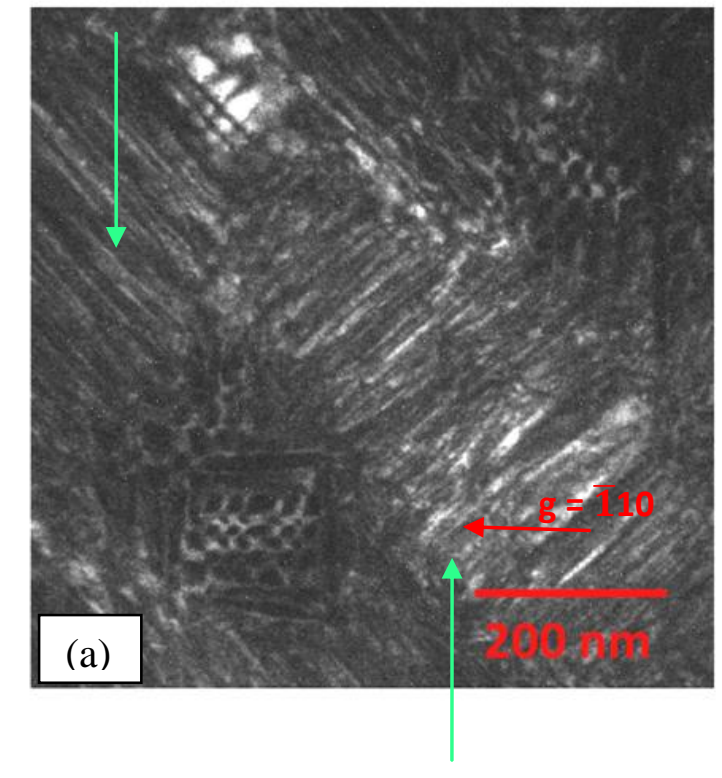

$\mathrm{Co}_{40.2} \mathrm{Pt}_{59.8}:$ DF Image, $\mathrm{g}=110$

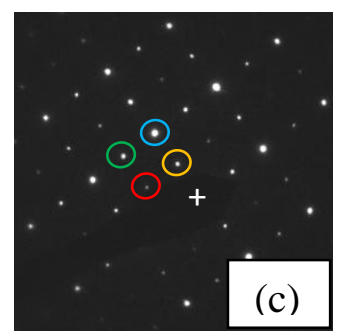

Figure 4.4: 110 DF images comparing (a) $\mathrm{Co}_{40.2} \mathrm{Pt}_{59.8}$ and (b) $\mathrm{Co}_{38.8} \mathrm{Pt}_{61.2}$ samples, both continuously cooled from 750 to $650{ }^{\circ} \mathrm{C} @ 40{ }^{\circ} \mathrm{C} /$ day and then water quenched. Green arrows indicate side-on chessboards in (a). [001] SADPs from the regions imaged in (a) and (b) are shown in (c) and (d) respectively. Red circles indicate 110 type superlattice reflections, yellow circles indicate 010 type superlattice reflections, green circles indicate 100 type superlattice reflections and blue circles indicate fundamental reflections. The beam blocker indicates the zero beam, which has been additionally indicates by a white crosshair. 


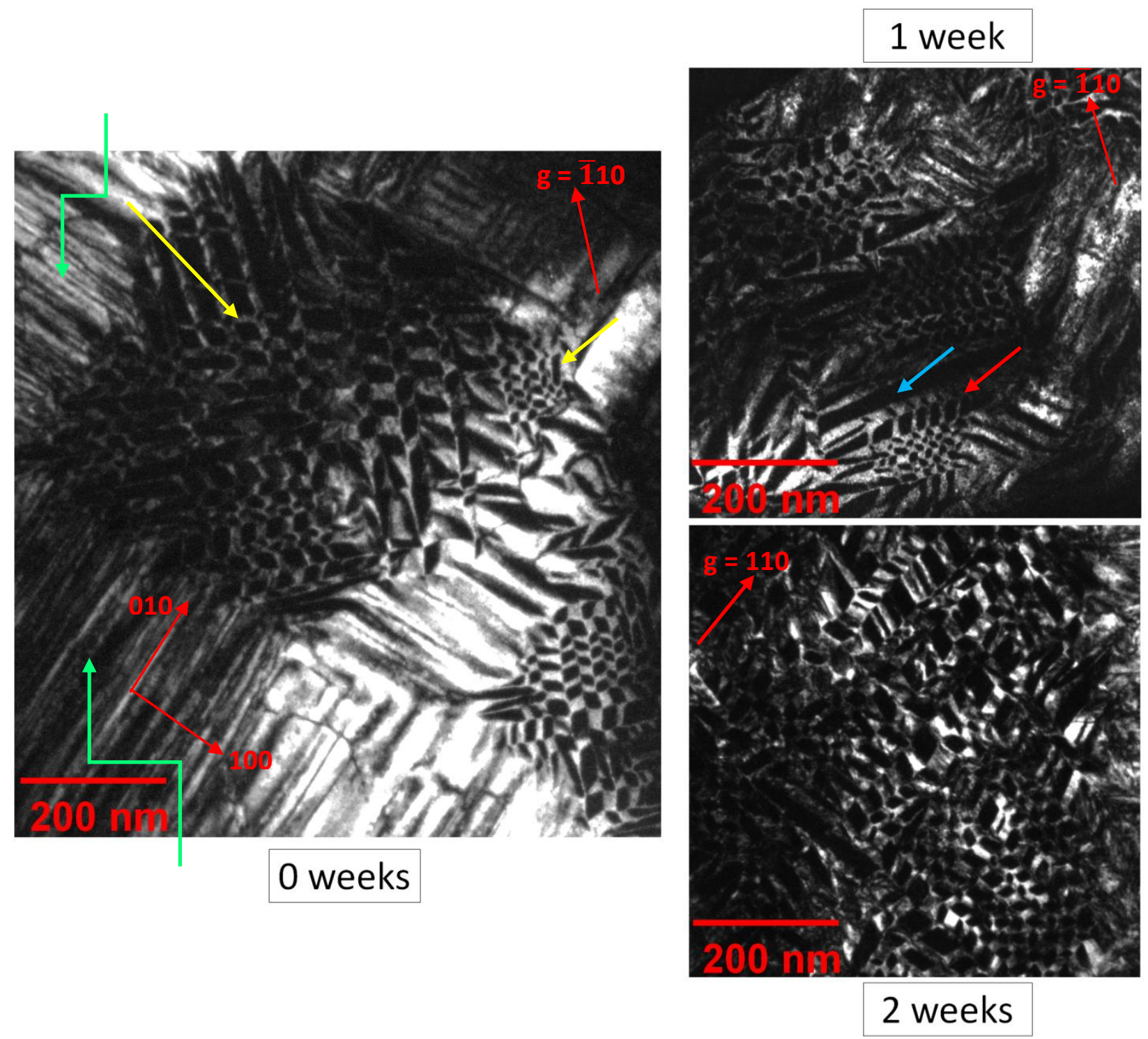

Figure 4.5: An overview of $110 \mathrm{DF}$ images from $\mathrm{Co}_{40.2} \mathrm{Pt}_{59.8}$ samples continuously cooled from 750 to $600{ }^{\circ} \mathrm{C} @ 40{ }^{\circ} \mathrm{C} /$ day and then annealed for 0,1 and 2 weeks at $600{ }^{\circ} \mathrm{C}$. In the sample annealed for 0 weeks, yellow arrows within the large colony point to sub-colonies while green arrows indicate side-on chessboard colonies. The blue arrow in the sample annealed for 1 week indicates an $\mathrm{L1}_{0}$ plate and red arrows indicate regions with altered tile geometries in samples annealed for 1 and 2 weeks. 

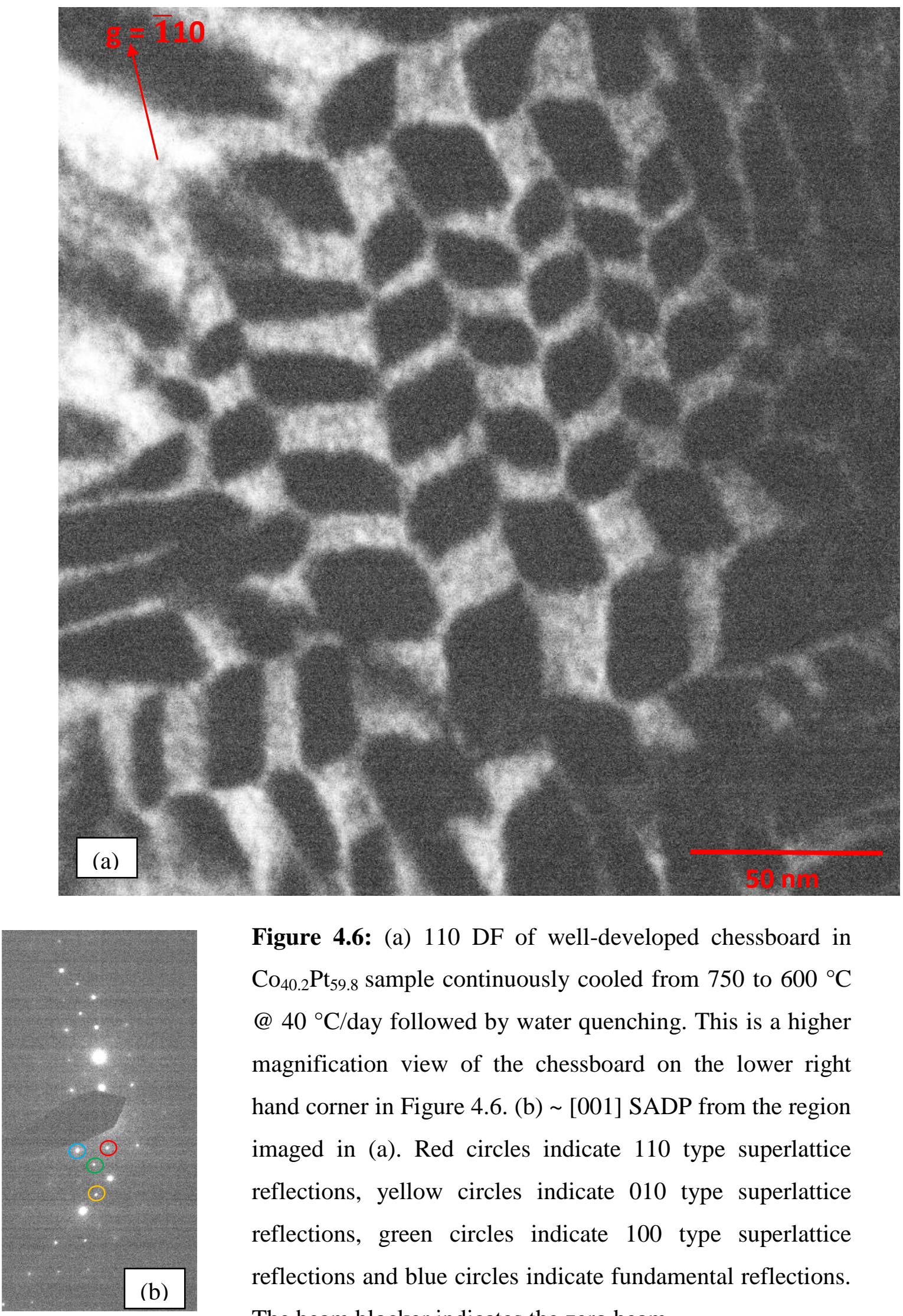

Figure 4.6: (a) 110 DF of well-developed chessboard in $\mathrm{Co}_{40.2} \mathrm{Pt}_{59.8}$ sample continuously cooled from 750 to $600{ }^{\circ} \mathrm{C}$ @ $40{ }^{\circ} \mathrm{C} /$ day followed by water quenching. This is a higher magnification view of the chessboard on the lower right hand corner in Figure 4.6. (b) [001] SADP from the region imaged in (a). Red circles indicate 110 type superlattice reflections, yellow circles indicate 010 type superlattice reflections, green circles indicate 100 type superlattice reflections and blue circles indicate fundamental reflections. The beam blocker indicates the zero beam. 
As discussed in Chapter 1, pseudospinodal decomposition begins with fluctuations in composition and order parameter which develop into the tweed structure, which in turn decomposes into the chessboard structure upon continued annealing. ${ }^{28}$ It is likely that the chessboard lengthscale and pattern is set during the quenching process, governed by the quench temperature and the cooling rate. Each chessboard colony is defined and discernible from neighboring colonies based on the direction of the nano-rod axis therein, which may be along one of the three cube axes. The chessboard colonies may possibly coarsen at the expense of colonies with orthogonal c-axis orientations, upon continued annealing. For instance, in the 0 week sample in Figure 4.5 (a), it appears that the regions in the colony indicated by yellow arrows in the big chessboard developed at different stages and that the smaller sub-colony just starting to form was previously part of a colony containing an edge-on chessboard variant. Alternately, the sub-colony might have developed from a region where the untransformed A1 phase persisted longer than in neighboring areas.

In the sample annealed for 1 week, the regions examined in Figure 4.5 and Figure 4.7 (a) showed colonies of chessboards separated by $\mathrm{L}_{0}$ plates, indicated by blue arrows and/or by regions evidencing altered tile geometries, indicated by red arrows. This latter type of region appears to evidence altered tile geometry; as is especially evident in Figure 4.7 (a). Herein, the $\mathrm{L}_{0}$ tiles are parallelogram - shaped with adjacent sides of different lengths unlike the rhombus-shaped tiles in the ideal chessboard structure. As shown in Figure 1.1 (Chapter 1), the rhombus-shaped tiles of the chessboard are symmetrical about the tile diagonals; however, this does not hold true for the altered tile geometry. 

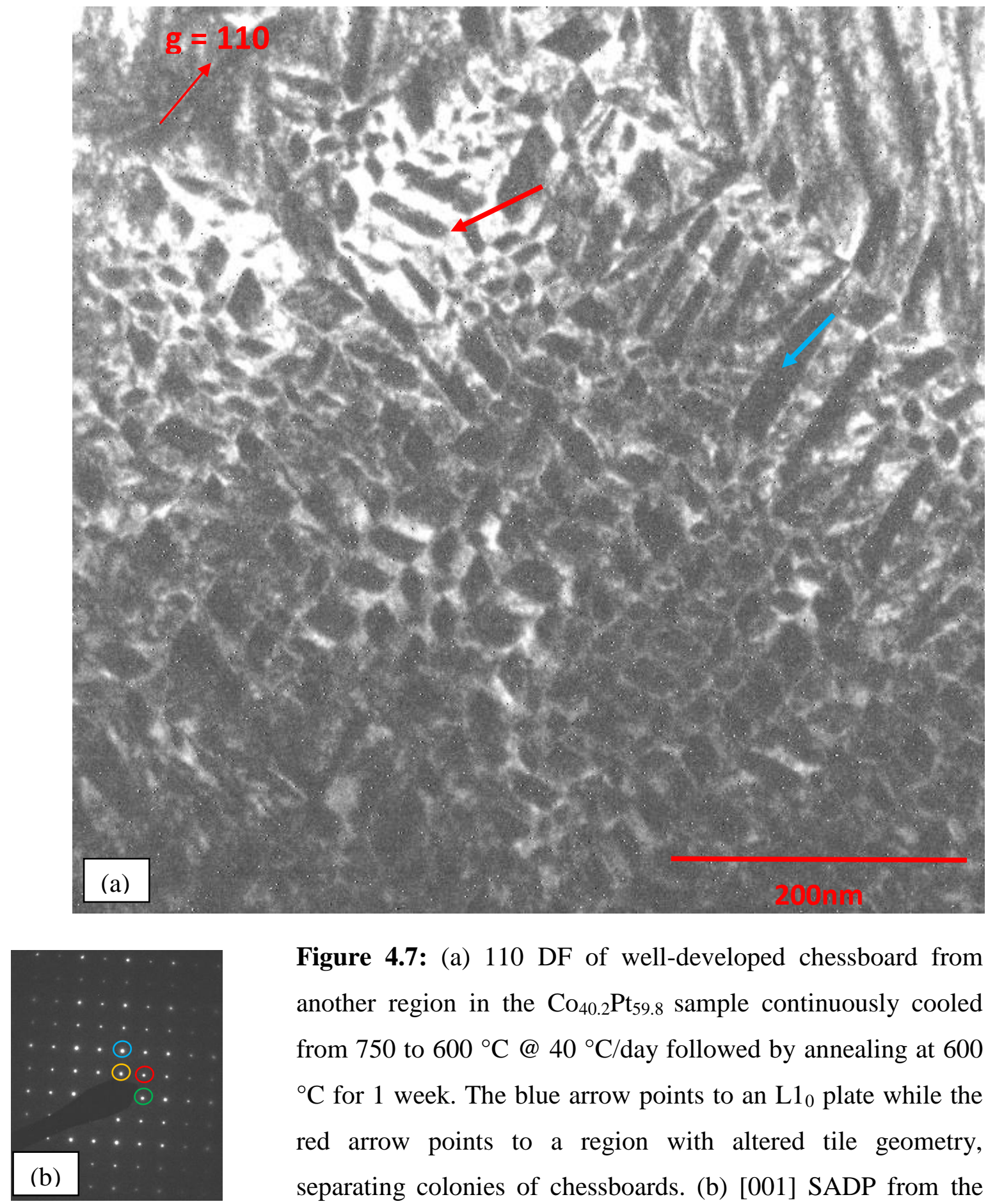

Figure 4.7: (a) 110 DF of well-developed chessboard from another region in the $\mathrm{Co}_{40.2} \mathrm{Pt}_{59.8}$ sample continuously cooled from 750 to $600{ }^{\circ} \mathrm{C} @ 40{ }^{\circ} \mathrm{C} /$ day followed by annealing at 600 ${ }^{\circ} \mathrm{C}$ for 1 week. The blue arrow points to an $\mathrm{L} 1_{0}$ plate while the red arrow points to a region with altered tile geometry, separating colonies of chessboards. (b) [001] SADP from the region imaged in (a). Red circles indicate 110 type superlattice reflections, yellow circles indicate 010 type superlattice reflections, green circles indicate 100 type superlattice reflections and blue circles indicate fundamental reflections. The beam blocker indicates the zero beam. 

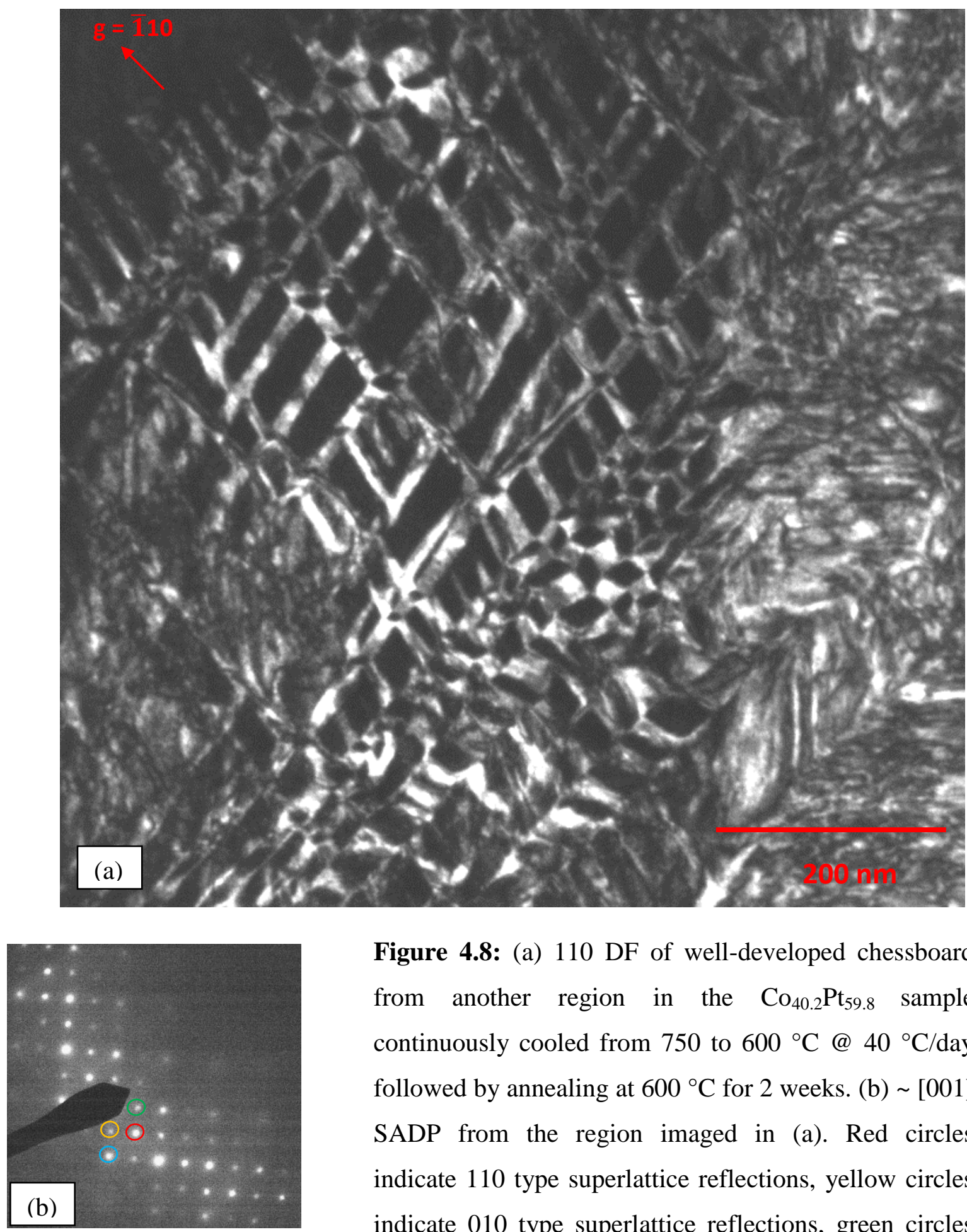

Figure 4.8: (a) 110 DF of well-developed chessboard from another region in the $\mathrm{Co}_{40.2} \mathrm{Pt}_{59.8}$ sample continuously cooled from 750 to $600{ }^{\circ} \mathrm{C} @ 40{ }^{\circ} \mathrm{C} / \mathrm{day}$ followed by annealing at $600{ }^{\circ} \mathrm{C}$ for 2 weeks. (b) [001] SADP from the region imaged in (a). Red circles indicate 110 type superlattice reflections, yellow circles indicate 010 type superlattice reflections, green circles indicate 100 type superlattice reflections and blue circles indicate fundamental reflections. The beam blocker indicates the zero beam. 
In the $\mathrm{Co}_{40.2} \mathrm{Pt}_{59.8}$ sample annealed for 2 weeks, the region examined in Figure 4.5 also indicated the distortion of the chessboard structure whereas the area in Figure 4.8 (a) revealed apparent broadening/breakdown of the chessboard structure, but clear trends with process conditions have not been isolated. However, it does appear that the chessboard tiling undergoes a change in geometry, gradually coarsening into plates, resulting in a disintegration of the chessboard structure with annealing times. It may be noted that identical heat treatment resulted in a wellformed chessboard microstructure in $\mathrm{Co}_{39.5} \mathrm{Pt}_{60.5}$ (Figure 1.10) as reported by Le Bouar et al. ${ }^{2}$ where the tiling was on a $20 \mathrm{~nm}$ lengthscale. On the other hand, even after an annealing time of 0 weeks, the tiling of our $\mathrm{Co}_{40.2} \mathrm{Pt}_{59.8}$ sample subjected to identical slow cooling was on a coarser length scale of $30-40 \mathrm{~nm}$.

A clear relationship between chessboard colony size, shape and process conditions has not been identified. It was observed, however, that the colony shape, was variable, ranging from equiaxed, as shown previously in this section, to banded. Images in 4.9 (a) and (b) show images of bandshaped chessboard colonies observed in the $\mathrm{Co}_{40.2} \mathrm{Pt}_{59.8}$ sample continuously cooled from 750 to $600{ }^{\circ} \mathrm{C} @ 40{ }^{\circ} \mathrm{C} /$ day followed by water quenching. APBs extending across several nanorods were frequently observed in the chessboard colonies viewed side-on and may potentially impact the coercivity, as discussed in Chapter 1. APBs are indicated by red arrows Figure 4.9 (b). In order to conclusively establish that the contrast features are APBs requires $\mathbf{g . R}$ contrast analysis using the three superlattice reflections viz. 001,100 and 110, as shown by Leroux et al. ${ }^{1}$ Unfortunately, this was not done. However, a comparison of the morphology with the literature suggests that these features are indeed APBs. ${ }^{9,10,22,23,54}$ 

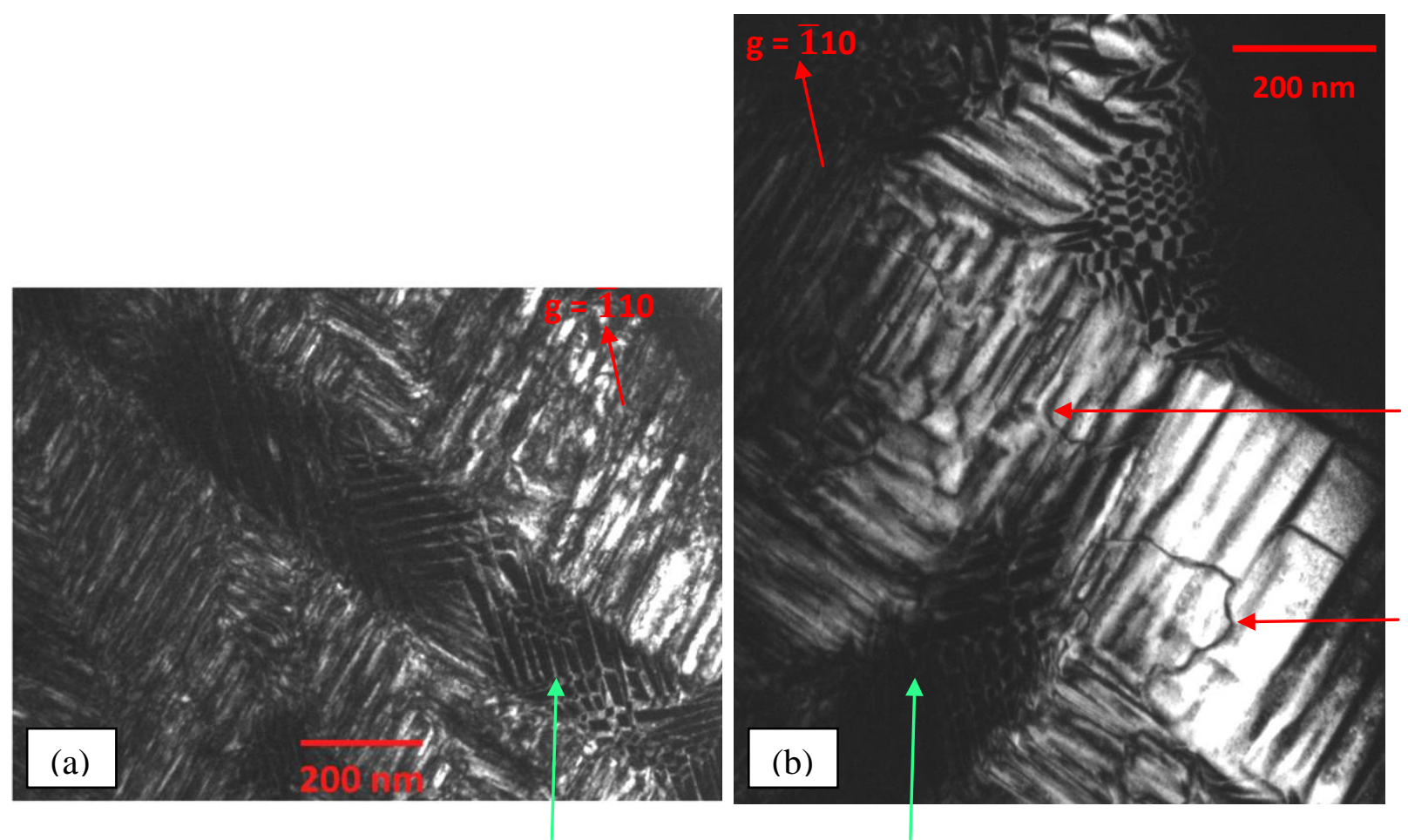

Figure 4.9: $110 \mathrm{DF}$ images $\mathrm{Co}_{40.2} \mathrm{Pt}_{59.8}$ sample continuously cooled from 750 to $600{ }^{\circ} \mathrm{C} @ 40$ ${ }^{\circ} \mathrm{C} /$ day followed by water quenzching highlighting $(\mathrm{a}, \mathrm{b})$ band-shaped chessboard colonies indicated by green arrows and (b) APBs indicated by red arrows.

Qualitatively different band-shaped colonies were also observed in the sample annealed for 2 weeks post-slow cooling, as shown in Figure 4.10 (a). The [001] SADP from this region, shown in Figure 4.10 (b), contained the 110, 001 and 100 superlattice reflections. In addition to exhibiting bright contrast in 001, 100 dark field images, the $\mathrm{L}_{2}$ phase, if present, would light up exclusively in the 110 dark field image. We note the absence of the $\mathrm{L} 1_{2}$ phase in the bands labeled 1 and 2 based on the lack of bright contrast in the corresponding 110 dark field image in Figure 4.10 (a). While equiaxed chessboard colonies occur in other regions of this sample, these bands appear to be comprised of $\mathrm{L} 1_{0}$ plates in the classic polytwin configuration ${ }^{22,27}$. It may be useful to note here that two $\mathrm{L} 1_{0}$ orientation variants are not in contact in the chessboard structure, unlike in the polytwinned structure.

Despite some apparent similarities between the structures shown in Figs. 4.9 (a) and 4.10, these in fact are very different. This variation of microstructure within the same TEM sample are again 
consistent with local variations in composition on the order of the tens-hundreds of microns, as discussed above, since an all- $\mathrm{L1}_{0}$ microstructure implies a Co-rich region outside the two-phase coexistence region of the phase diagram. It may be pointed out that the sample used for TEM was processed separately from the material used for investigation by XRD and VSM. With XRD and TEM both hinting at inhomogeneity, even greater efforts are required to accomplish homogenization of future samples.
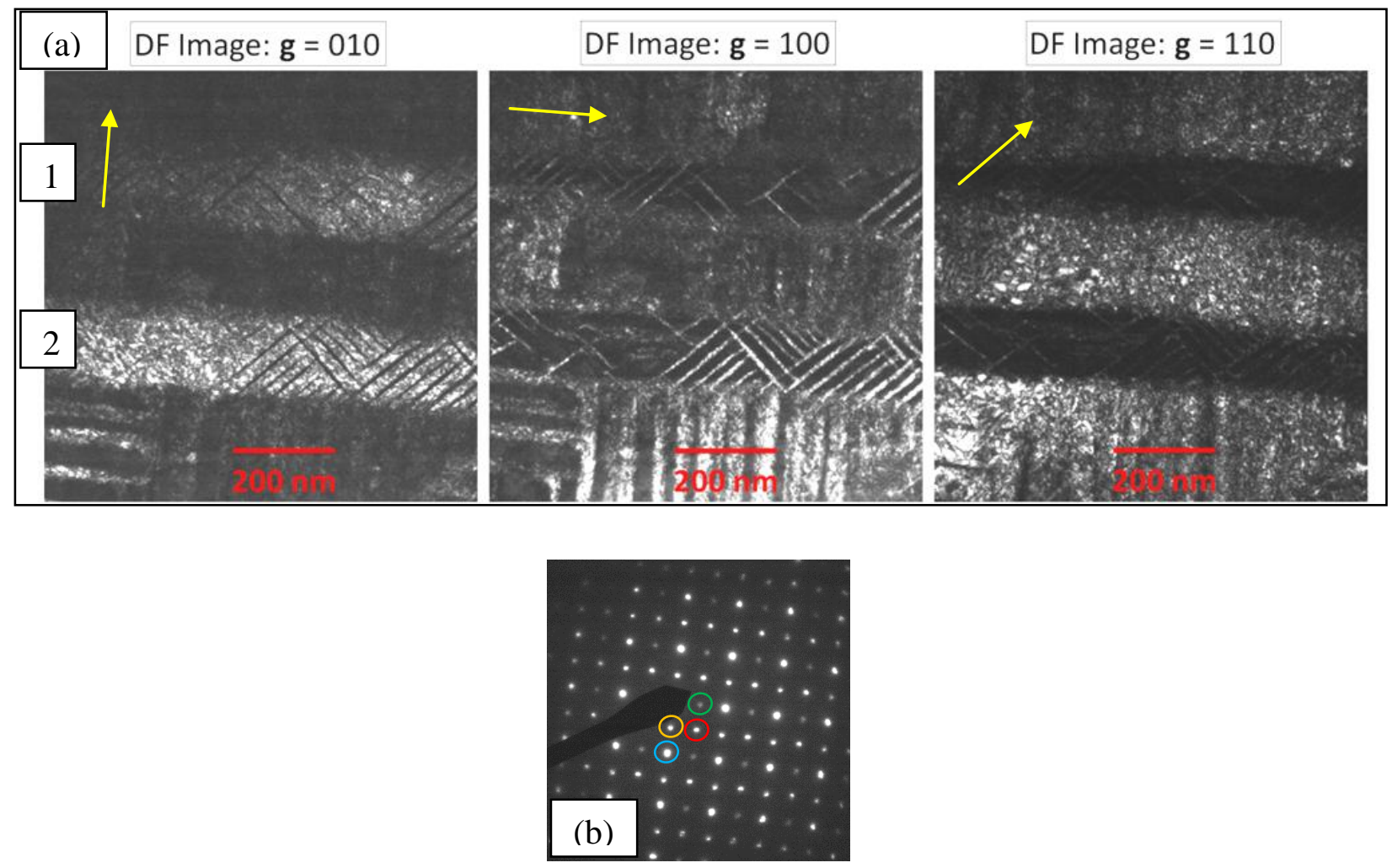

Figure 4.10: (a) 010, 100 and 110 DF images of band-shaped colonies in $\mathrm{Co}_{40.2} \mathrm{Pt}_{59.8}$ samples continuously cooled to $600{ }^{\circ} \mathrm{C}$ and annealed for 2 weeks (b) [001] SADP from the region imaged in (a). 


\subsection{Eutectoid transformation temperature: DSC}

DSC was used to determine the $\mathrm{A} 1 \rightarrow \mathrm{L} 1_{0}+\mathrm{L1}_{2}$ eutectoid transformation temperature in Co-Pt by detecting caloric changes during controlled heating and cooling experiments. Herein, a $\mathrm{Co}_{40.2} \mathrm{Pt}_{59.8}$ sample with chessboard microstructure obtained upon gradual cooling from 750 to $650{ }^{\circ} \mathrm{C} @ 40{ }^{\circ} \mathrm{C} /$ day followed by water quenching, was heated/cooled @ $10{ }^{\circ} \mathrm{C} / \mathrm{min}$ and corresponding variations of heat flow with temperature were monitored. From this thermal analysis, the experimentally determined eutectoid transformation temperature appeared to be $\approx$ $760{ }^{\circ} \mathrm{C}, \approx 30^{\circ} \mathrm{C}$ higher than the published value of $730^{\circ} \mathrm{C}^{1}$.

The DSC plots obtained from the heating experiments cannot be used for accurately determining the onset of the transformation due to "pre-disordering" resulting from a reduction in the longrange order parameter, which appears as an endothermic signal prior to the nucleation of the disordered phase. The cooling experiments however do not face the above complication. Reproducible plots were obtained upon repeating the cooling experiments, as shown in Figure 4.11. The onset of the disorder to order transition upon cooling was determined by drawing a sloped straight line from the peak down to the baseline. The onset seems to be around $760{ }^{\circ} \mathrm{C}$ in both cases. It is likely that this result does not capture the equilibrium temperature and that the transformation requires some undercooling to proceed at an appreciable rate. Hence, the eutectoid cannot be lower than $760{ }^{\circ} \mathrm{C}$, although it could possibly be higher. Note that the cooling rates used in the DSC are 360 times larger than those used to promote the chessboard formation in our furnace. Hence, it will be unlikely we can extract a meaningful heat of ordering from the DSC data, but the estimation of the ordering temperature $\geq 760^{\circ} \mathrm{C}$ should still be valid.

This implies that our current 'chessboard' samples, which were produced by slow cooling the A1 base material from $750{ }^{\circ} \mathrm{C}$, were not heated fully above this higher eutectoid temperature before beginning to slow cool. The ramp started $10{ }^{\circ} \mathrm{C}$ below the transition temperature, within the $\mathrm{L}_{0}$ $+\mathrm{L1}_{2}$ two phase region, and the decomposition would have likely started immediately. Thus, the alloys were not continuously cooled through the eutectoid. While this may not be important, it may affect the wavelength of the fluctuations leading to the tweed structure and ultimately, the chessboard structure, thereby affecting the final microstructural lengthscale and the corresponding magnetic properties. Interestingly, we do not see pro-eutectoid microconstituents forming, which may be a benefit of actually not going above the isotherm temperature. 
Processing samples via slow cooling from above the true eutectoid, say from $800{ }^{\circ} \mathrm{C}$, instead of from $750{ }^{\circ} \mathrm{C}$, would allow us to verify this.

From our preliminary results, the small exothermic peak at $355{ }^{\circ} \mathrm{C}$ appears to correspond well to the Curie temperature of the $\mathrm{L}_{2}$ phase determined in Chapter 3. However, it is not understood why an additional peak corresponding to the Curie temperature of the $\mathrm{L} 1_{0}$ phase is not obtained. The single broad Curie temperature peak in the AC susceptibility versus temperature curves for $\mathrm{L}_{0}+$ FCC CoPt observed by Xiao et al. ${ }^{55}$ suggests that there may be a broad distribution of Curie temperatures, rather than distinct peaks corresponding to individual phases in the 0 week sample investigated by this technique, as the order parameters undergo evolution in all phases present. Further work is needed to verify both the eutectoid temperature and the Curie temperature. 

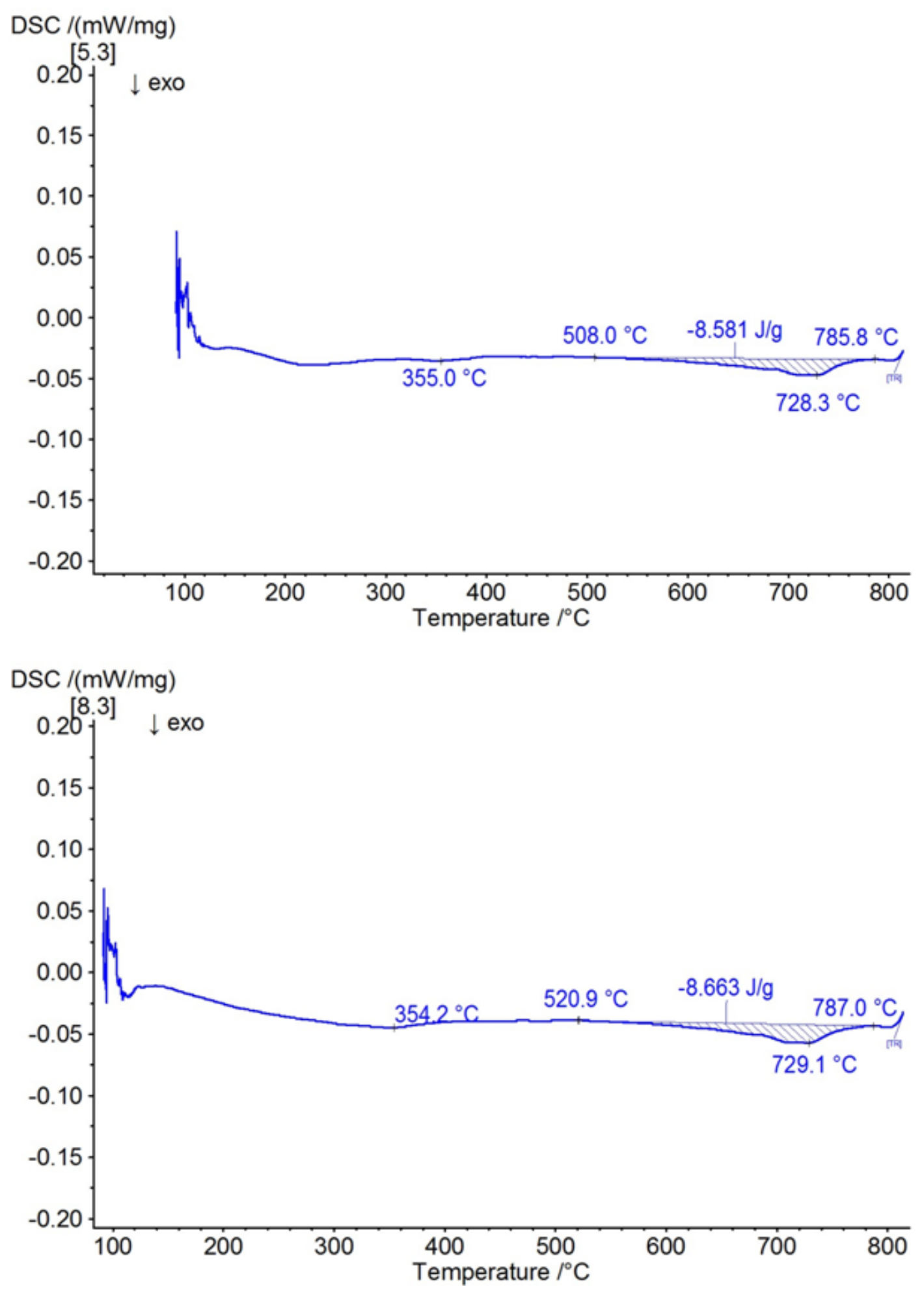

Figure 4.11: Heat flow versus temperature plots from two DSC cooling experiments performed on a $\mathrm{Co}_{40.2} \mathrm{Pt}_{59.8}$ sample originally processed via slow cooling to $650{ }^{\circ} \mathrm{C}$, followed by water quenching. 


\subsection{Magnetic Properties}

\subsubsection{Effect of annealing time}

$\mathrm{Co}_{40.2} \mathrm{Pt}_{59.8}$ samples continuously cooled from 750 to $600{ }^{\circ} \mathrm{C}$, and then quenched ("0 week") or annealed at $600{ }^{\circ} \mathrm{C}$ for 1 week and 2 weeks were verified to exhibit the chessboard microstructure using TEM. It may be noted that the latter sample was actually annealed for 15 days, following Le Bouar et al. ${ }^{2}$. For ease of designation and comparison with the samples annealed for 0 and 1 weeks, we have referred to the annealing duration of this sample as ' 2 weeks'. A comparison of the magnetic properties of these samples is shown in Figure 4.12. From this figure, we see that annealing at $600{ }^{\circ} \mathrm{C}$ for one week produces a local maximum in the coercivity, remanence, remanence to saturation ratio as well as the maximum energy product; and a local minimum in saturation magnetization. 


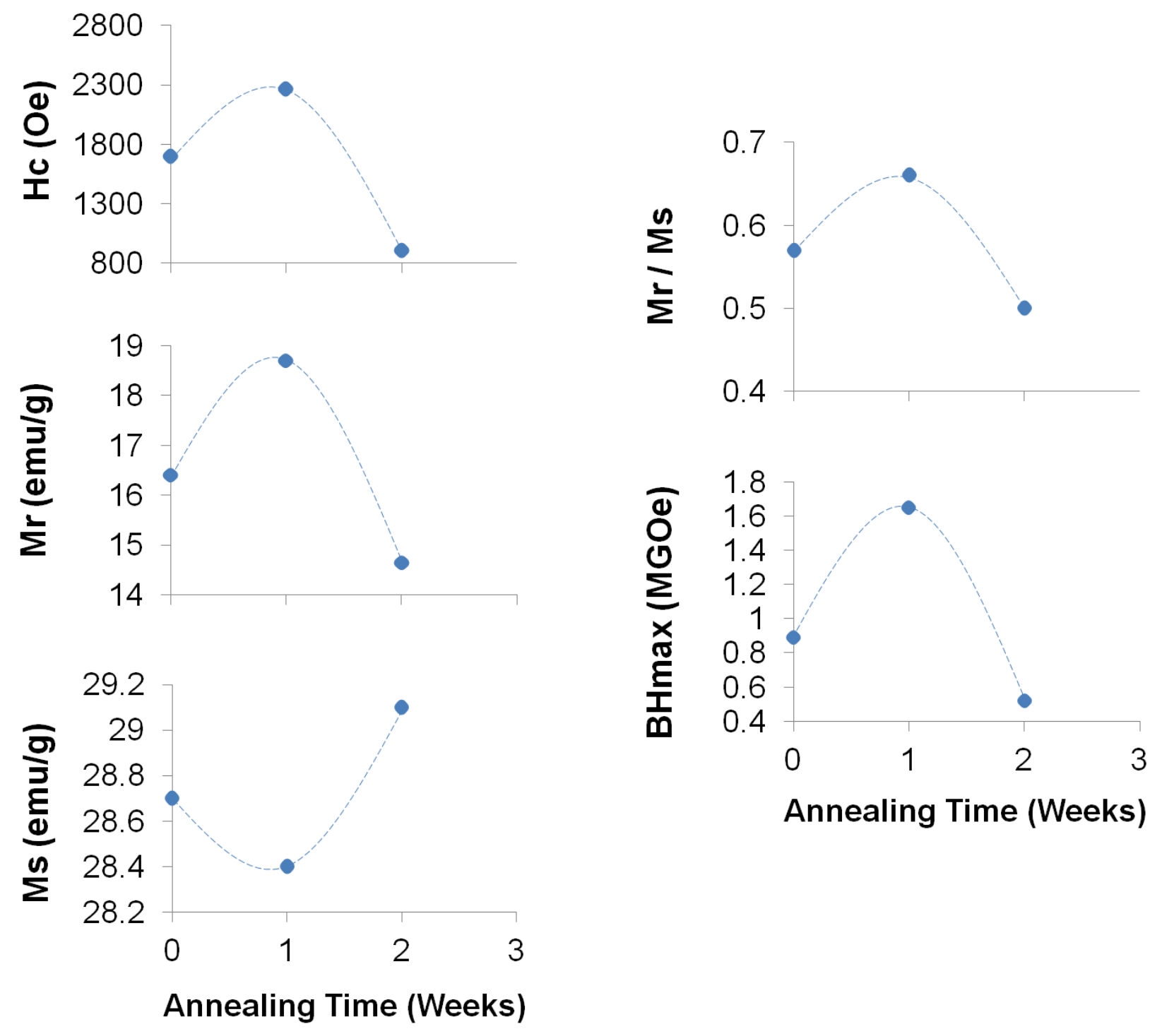

Figure 4.12: Variation of magnetic properties with annealing time at $600{ }^{\circ} \mathrm{C}$ in continuously cooled $\mathrm{Co}_{40.2} \mathrm{Pt}_{59.8}$ 'chessboard' samples. The dashed lines are guides to the eye.

The initial magnetization behavior of the peak hardness $\mathrm{Co}_{40.2} \mathrm{Pt}_{59.8}$ material obtained upon slow cooling followed by annealing at $600{ }^{\circ} \mathrm{C}$ for 1 week is shown in Figure 4.13. The initial magnetization behavior lends insight into the mechanisms for magnetization reversal and the curve appears commensurate with domain wall pinning. 


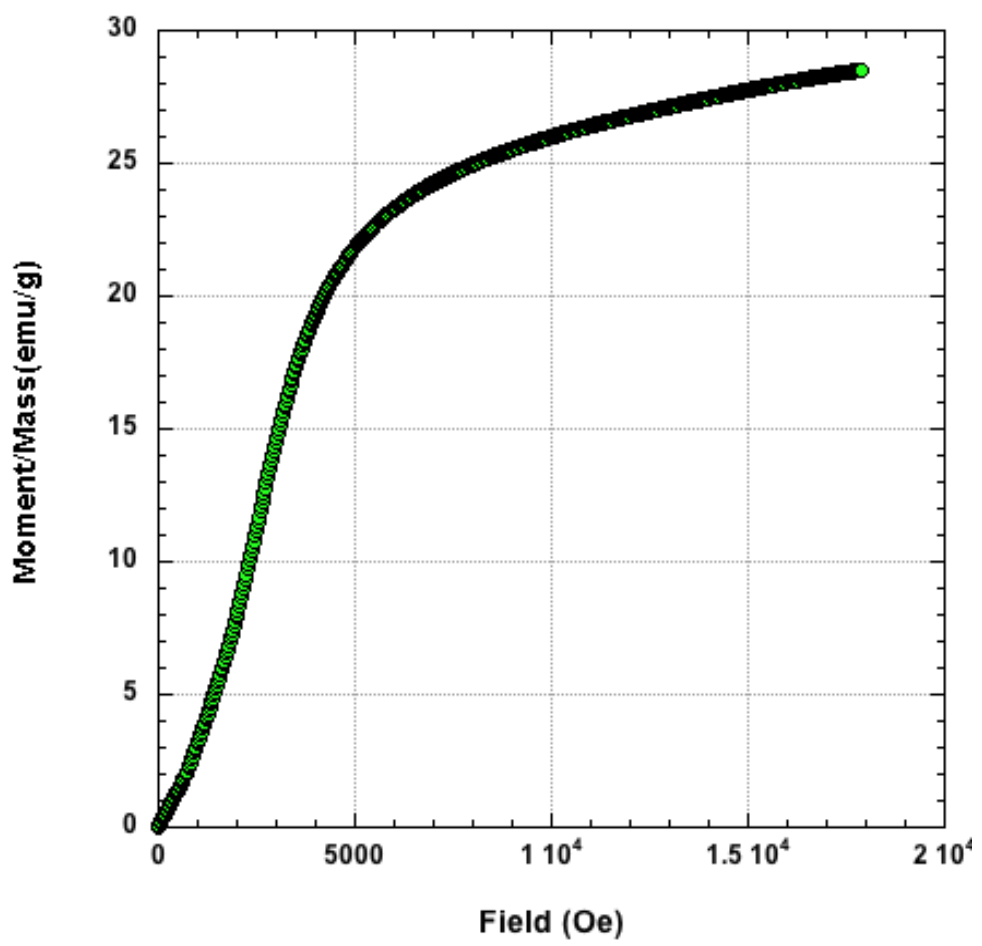

Figure 4.13: Initial magnetization curve for $\mathrm{Co}_{40.2} \mathrm{Pt}_{59.8}$ sample continuously cooled from 750 to $600{ }^{\circ} \mathrm{C}$ and then held at $600{ }^{\circ} \mathrm{C}$ for 1 week, resulting in maximum coercivity.

The hysteresis loops from these continuously cooled $\mathrm{Co}_{40.2} \mathrm{Pt}_{59.8}$ samples annealed at $600{ }^{\circ} \mathrm{C}$ for various time durations are shown in Figure 4.14. Corresponding susceptibility $(\mathrm{dM} / \mathrm{dH})$ versus magnetic field curves are plotted in Figure 4.15. A peak in the susceptibility suggests the presence of a magnetic phase undergoing reversal. 

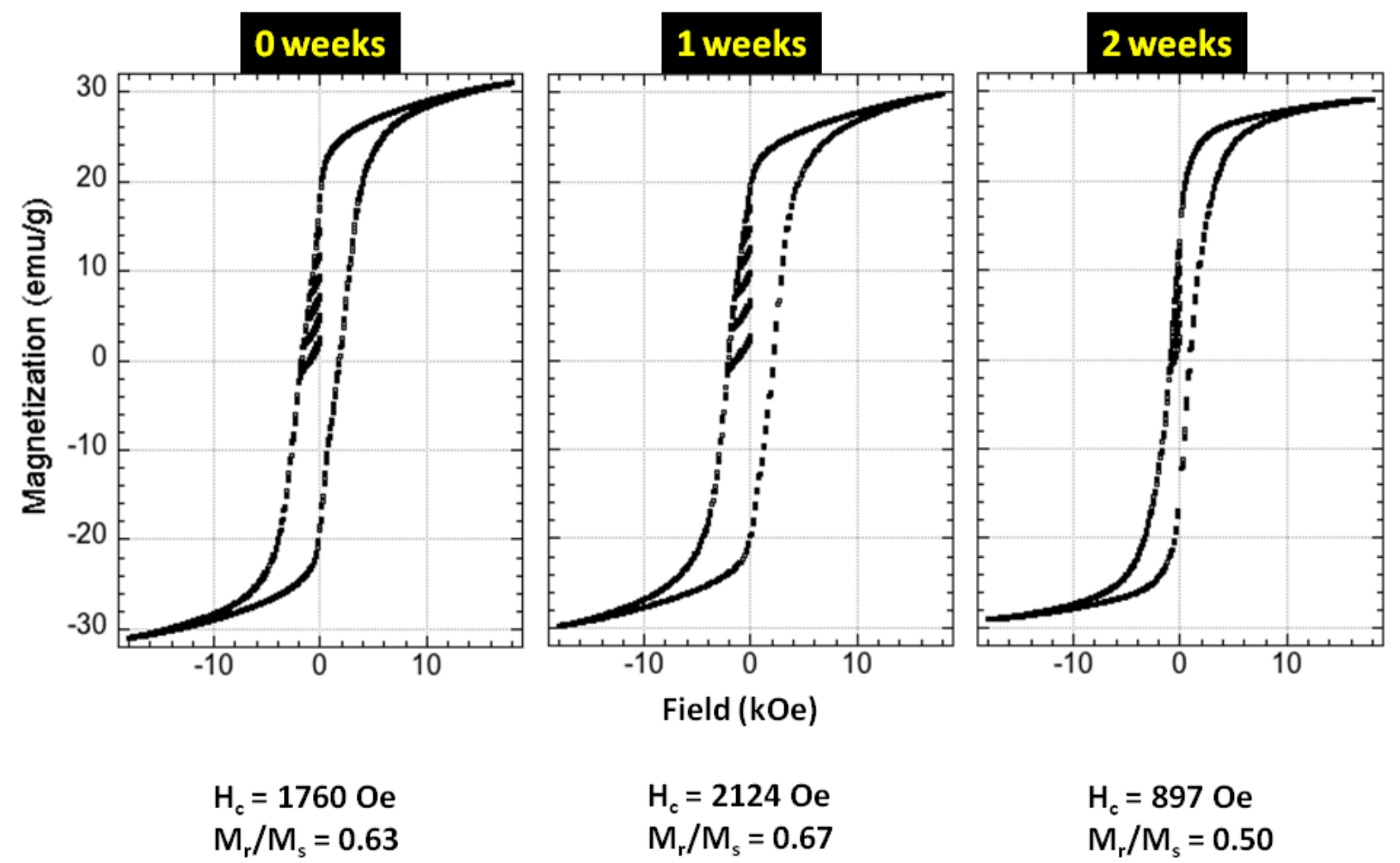

Figure 4.14: Major $\mathrm{M}-\mathrm{H}$ loops in $\mathrm{Co}_{40.2} \mathrm{Pt}_{59.8}$ samples continuously cooled to $600{ }^{\circ} \mathrm{C}$ and annealed for 0,1 or 2 weeks. Minor "recoil" loops are also shown. The coercivity and remanence ratio for each sample is also summarized.

In Figure 4.15, the susceptibility corresponding to an annealing time of 0 weeks showed a peak indicating the reversal of the soft phase, marked by a blue arrow, near zero field. A smaller peak indicating the reversal of the hard phase, marked by a green arrow, was observed above the overall $\mathrm{H}_{\mathrm{c}}$. This indicates that the major volume fraction consists of the softer phase which is uncoupled and readily switches; whereas the minor fraction of hard phase reverses after $\mathrm{H}_{\mathrm{c}}$. 

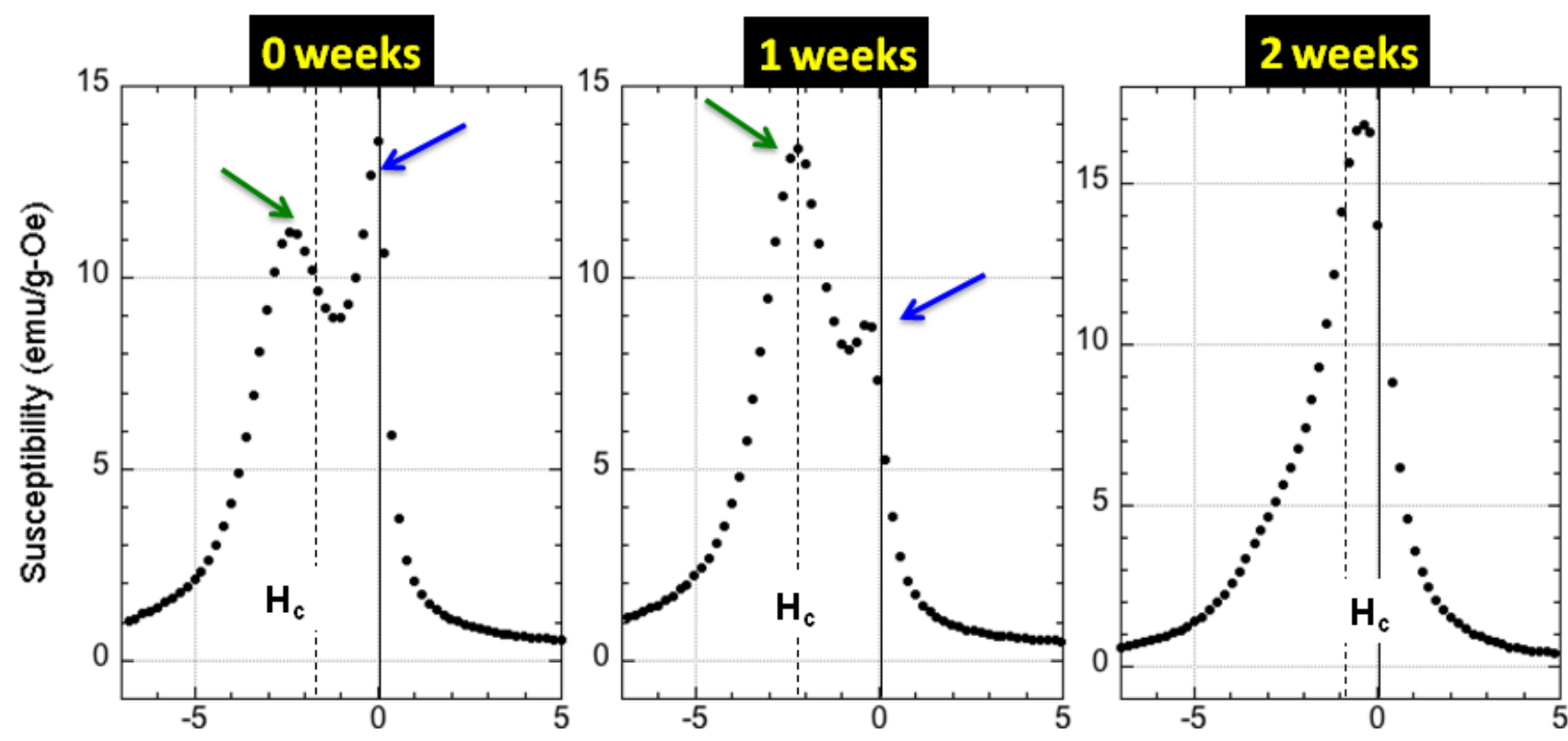

Field (kOe)

Figure 4.15: Susceptibilities obtained during magnetization reversal from positive to negative saturation, corresponding to major M-H loops shown in Figure 4.14.

An annealing period of 1 week resulted in a susceptibility curve similar to that obtained after 0 weeks. For the case of the sample annealed for 1 week, however, the soft peak near zero field, was smaller than the hard peak, which is now coincident with the coercivity. Thus, this sample appeared to contain a greater volume fraction of the hard phase compared to the decoupled soft A1 phase, consistent with the XRD volume fraction analysis present in subsection 4.1.1.

It is possible that the hard phase obtained upon annealing for 1 week may be "harder" than at 0 weeks, i.e., more ordered, leading to a higher magnetocrystalline anisotropy. This would explain the higher remanence ratio observed for the sample annealed for 1 week versus 0 weeks based on the fact that the $\mathrm{L} 1_{2}$ phase now couples to a more ordered $\mathrm{L} 1_{0}$ phase.

After annealing for 2 weeks, the resulting $\mathrm{M}(\mathrm{H})$ curve shows only a single peak, with a shape that is consistent with Kneller and Hawig's ${ }^{38}$ example of an overaged but exchange-coupled composite, wherein the demagnetization curve is concave from below the remanence to the saturation in reverse field, as shown in Figure 4.16. Overaging results in reduced coercivity as 
the spatial extent and volume fraction of the soft phase becomes larger enough to permit reversed-domain nucleation at relatively low fields that assists reversal of the minority hardphase region. However, there must still be significant exchange-coupling between the hard and soft phases, since separate peaks in the susceptibility are not observed.
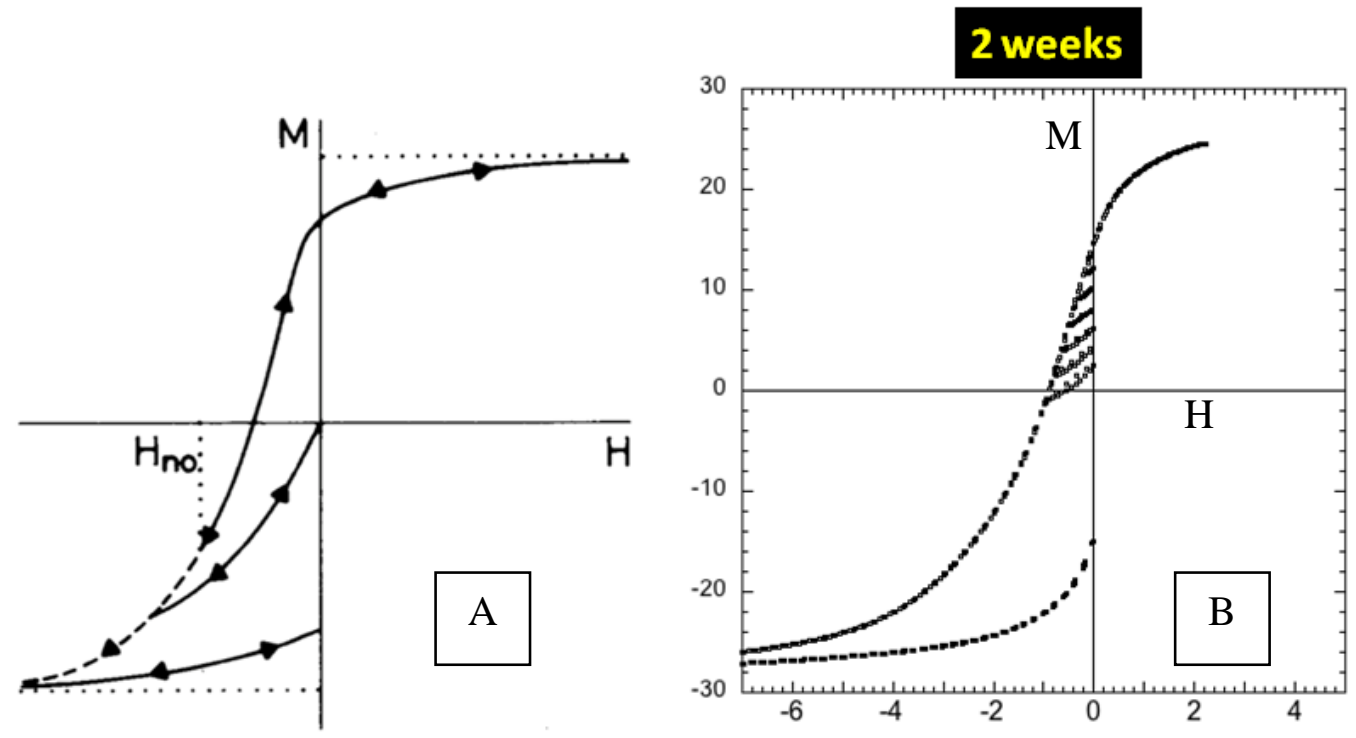

Figure 4.16: Comparing (A) the deduced $\mathrm{M}-\mathrm{H}$ loop from a coupled but overaged sample as published by Kneller and Hawig ${ }^{38}$ (B) the M-H loop from our $\mathrm{Co}_{40.2} \mathrm{Pt}_{59.8}$ sample annealed for 2 weeks.

An analysis of the magnetic susceptibility curves in Figure 4.15 had suggested that there was a smaller volume fraction of the hard phase and a larger volume fraction of the decoupled soft phase, in the sample annealed for 0 weeks. Further progress in phase transformation after an annealing time of 1 week resulted in a decrease in the volume fraction of the decoupled soft phase. The uncoupled residual A1 phase was completely annealed out after a duration of 2 weeks. This analysis based on the magnetic susceptibility curves is thus entirely consistent with the above volume fraction analysis.

The recoil loops, used to check for reversibility of the magnetization are shown in an enlarged view in Figure 4.17. These are a series of minor loops obtained in the second and third quadrant, for different values of the reverse field, $\mathrm{H}_{\text {reverse }}$. Figure 4.18 displays a schematic illustration 
showing the quantities of interest, such as the recoverable component of the magnetization $\mathrm{M}_{\text {rec}}$, the irreversible component, $\mathbf{M}_{\text {irrev }}$ and their sum, $\mathbf{M}_{\text {total }}$. The recoverable magnetization is a proxy for the true reversible magnetization, which is highly non-trivial to extract, and $\mathrm{M}_{\mathrm{rec}}$ still serves as a useful indicator of exchange-spring magnetism ${ }^{56}$. For a perfectly reversible magnet, the minor loops would closely retrace the major loop. Exchange-spring magnets should exhibit large $\mathrm{M}_{\mathrm{rec}}$ and recoverable susceptibility $\chi_{\mathrm{rec}}$, since the soft phase, while reversing more readily in the second quadrant, springs back into alignment with the hard phase upon removal of the applied field. However, the recoil loops in Figure 4.17 show a relatively low recovery of magnetization; the materials appear not to be recovering major portions of their magnetization. This suggests that the magnetization in the soft phase is irreversibly switched. The recoverable remanence and saturation magnetization are plotted for the three samples annealed at $600{ }^{\circ} \mathrm{C}$ in Figure 4.19 (A) and (B) respectively. The best $\mathrm{M}_{\mathrm{rec}} / \mathrm{M}_{\mathrm{r}}$ we observe is 0.23 , compared with values of 0.35 obtained in exchange-coupled $\mathrm{Nd}_{2} \mathrm{Fe}_{14} \mathrm{~B}+\alpha$-Fe by Harland, et al., at the corresponding coercive field. ${ }^{56}$ The best value of $\mathrm{M}_{\mathrm{rec}} / \mathrm{M}_{\mathrm{s}}$ we observed is 0.13 , compared with 0.5 obtained by Feutrill, et al. ${ }^{57}$
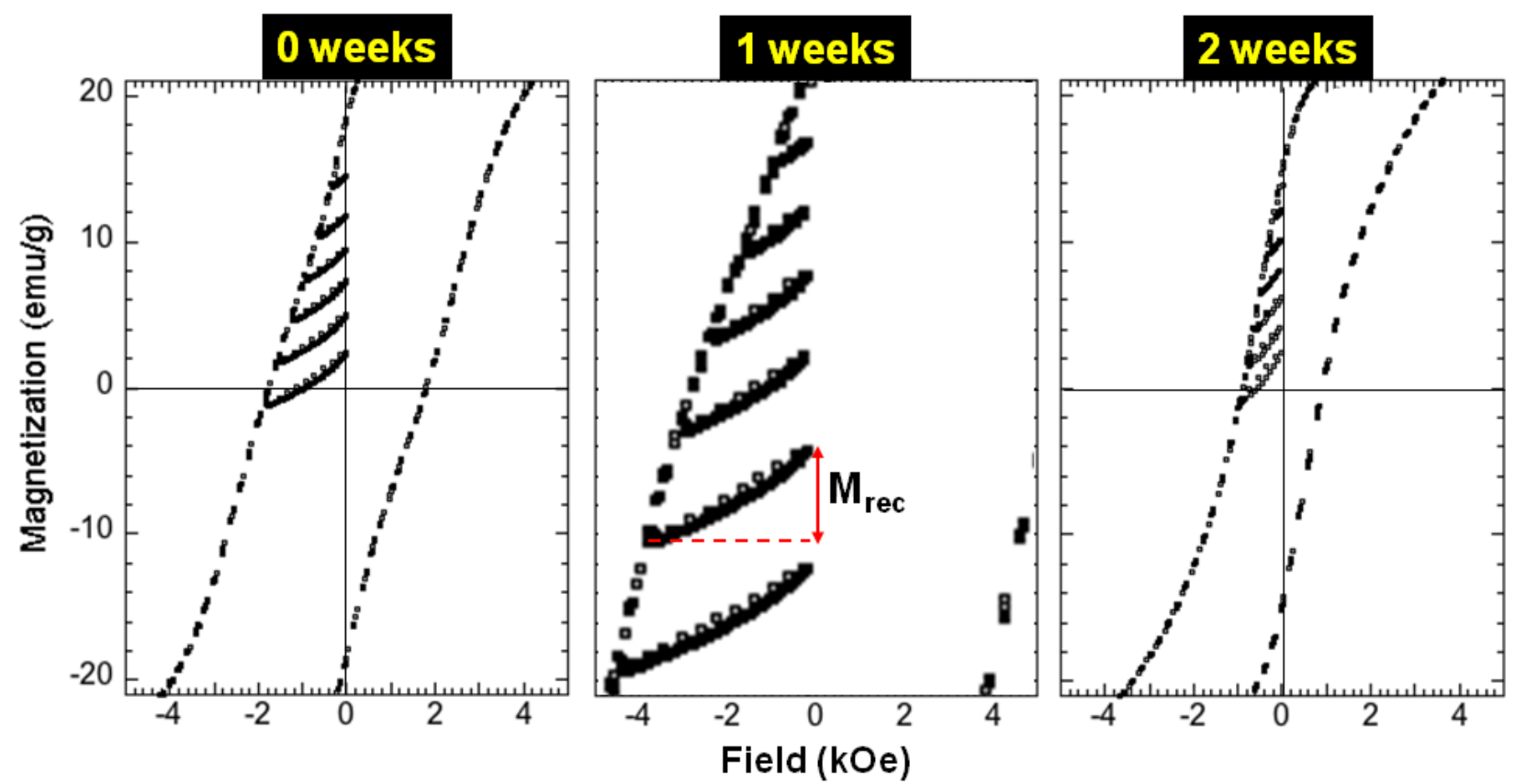

Figure 4.17: Enlarged view of relevant section from Figure 4.14, with an emphasis on the recoil loops. 


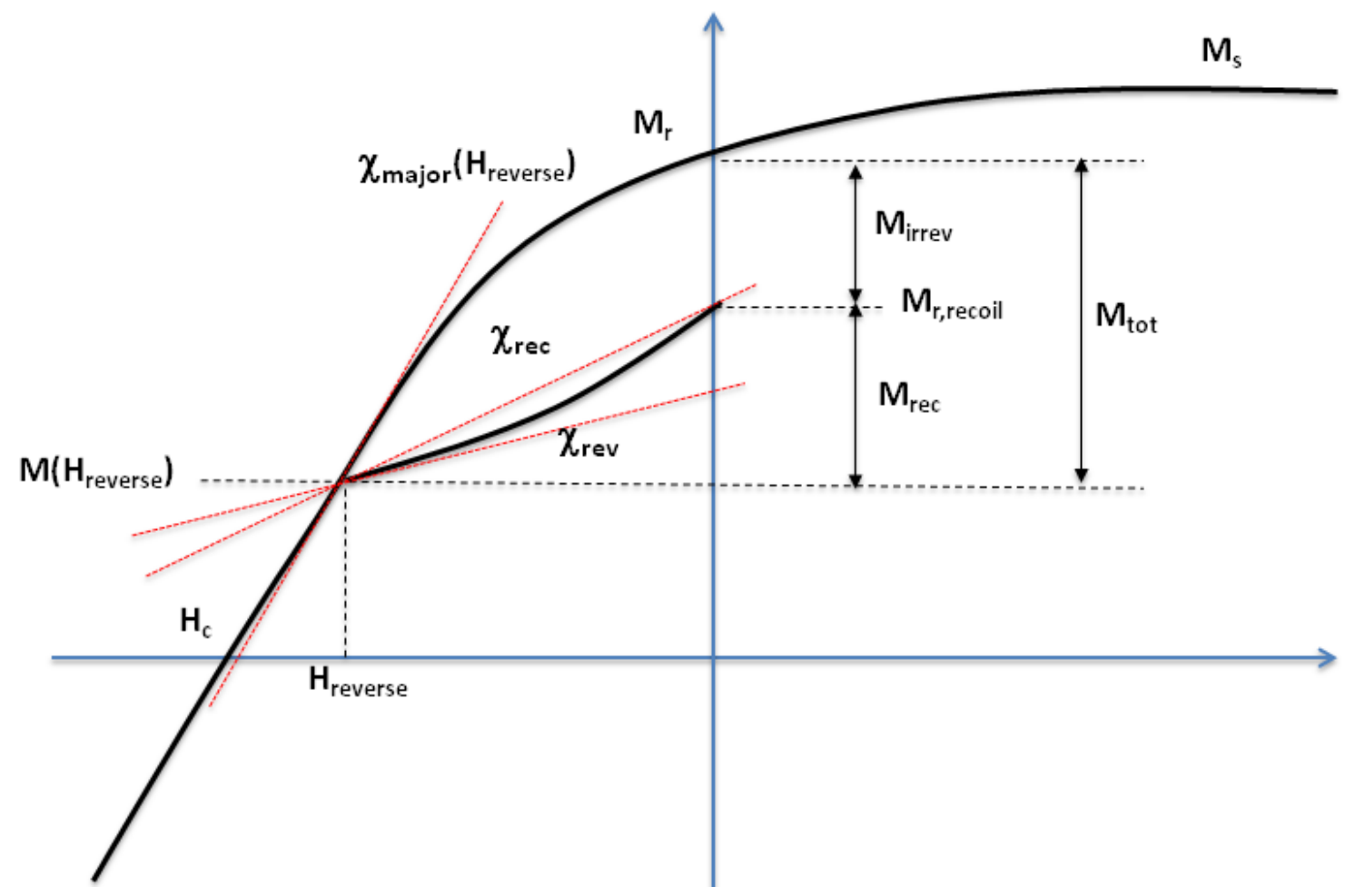

Figure 4.18: A schematic demagnetization curve and recoil loop.
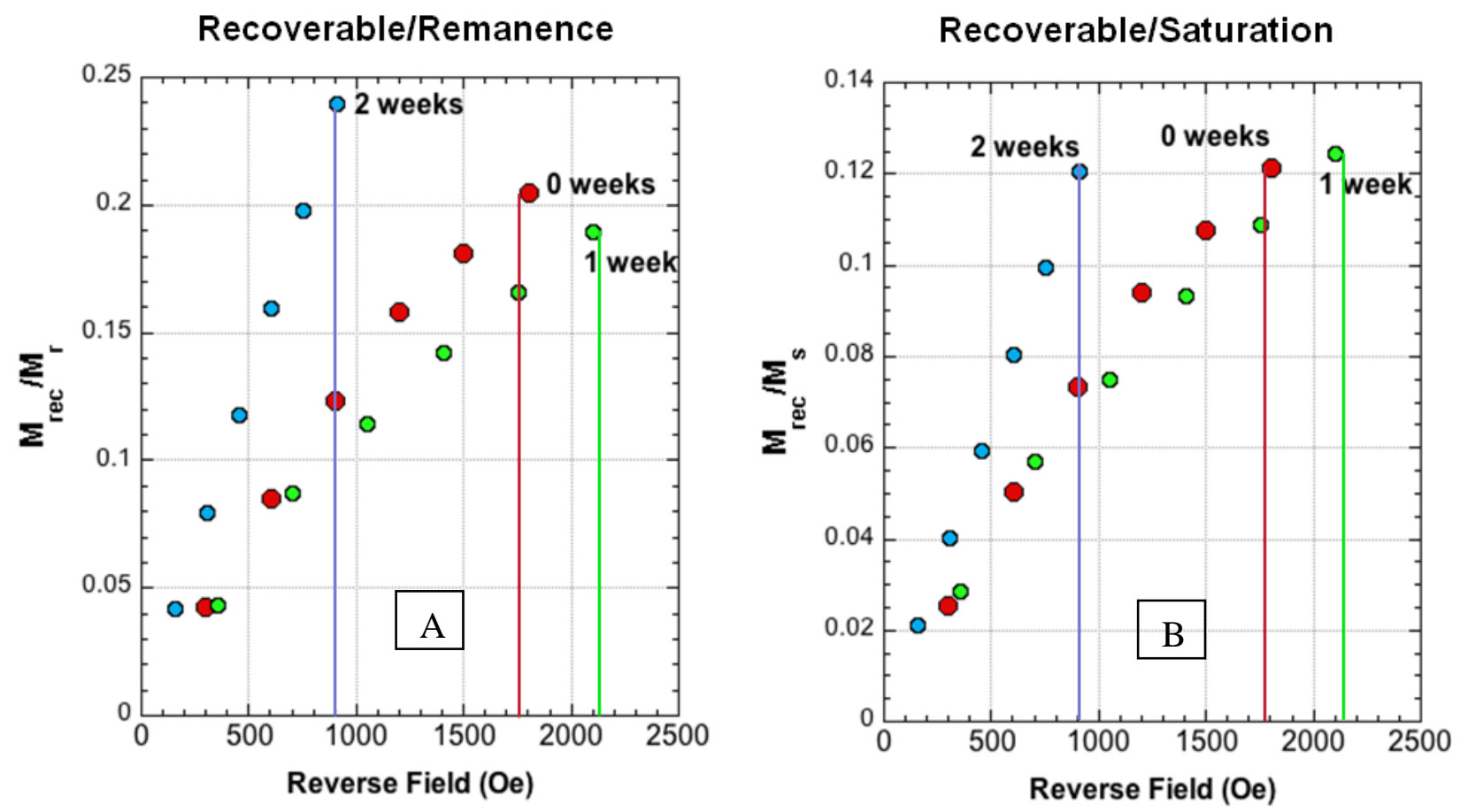

Figure 4.19: (A) The recoverable remanence $M_{r e c} / M_{r}$ and (B) The recoverable saturation $M_{\text {rec }}$ / $\mathrm{M}_{\mathrm{S}}$. 
We had expected nanochessboards to exhibit a greater degree of exchange spring behavior. The 2 week annealed sample exhibits an $\mathrm{M}(\mathrm{H})$ loop consistent with exchange-coupling since there are two phases and one peak, but with an overaged microstructure. From XRD, this sample is known to contain only $\mathrm{L}_{0}$ and $\mathrm{L}_{2}$ with no residual $\mathrm{A} 1$ phase, so presumably the coupling is between the two ordered phases. Nonetheless, the recoverable magnetization is relatively small. The samples annealed for 0 and 1 week both also show evidence for exchange coupling based on the susceptibility curves, since there are three phases present based on the XRD analysis, but 2 peaks in the hysteresis curves. The presence of these two peaks indicates that there is an uncoupled soft phase corresponding to the soft peak in addition to the coupled phases comprising the the chessboard which contribute to the hard peak in the dM/dH curves shown in Figure 4.15. Note, however, that XRD shows that these samples contain significant residual A1, along with $\mathrm{L}_{0}+\mathrm{L1}_{2}$. We believe that the uncoupled soft phase is the residual $\mathrm{A} 1$, while the hard peak in the susceptibility arises from coupled $\mathrm{L}_{0}+\mathrm{L}_{2}$. The $\mathrm{A} 1$ phase may not be exchange-coupled because it lies in regions where it is not in intimate contact with $\mathrm{L1}_{0}$. A decoupled A1 phase is possible if it (a) is not part of the nanochessboard but instead lies in macroscopic volumes that are untransformed or (b) lies in mesoscopic volumes within colonies, such as near the interface between two nanochessboard colonies. Either situation would permit the A1 phase to not be in close contact with the $\mathrm{L} 1_{0}$ phase or to align geometrically with it. $\mathrm{The} \mathrm{L}_{2}$ and $\mathrm{L} 1_{0}$ phases may couple due to their intimate proximity in the chessboard. The 1 week annealed sample shows the largest remanence ratio of the chessboard samples we have examined, with XRD suggesting complete ordering of the $\mathrm{L}_{0}$ phase, but still the recoverable magnetization is small.

In addition to these chessboard specimens, we re-examined a subset of the $\mathrm{Co}_{41.7} \mathrm{Pt}_{58.3}$ samples discussed in Chapter 3. In particular, recoil loops were obtained from the sample annealed isothermally at $600{ }^{\circ} \mathrm{C}$ for 720 hours, which had a coercivity of 4000 Oe and a remanence ratio of 0.78 . The major loops susceptibility shows only single phase behavior, even though this sample consists of nanoscale $\mathrm{L}_{0}$ platelets embedded in an A1 matrix. However, we find that the recoverable magnetization is again quite small, of the same magnitude as the chessboard samples.

In summary, we find in all our multi-phase, hard/soft Co-Pt samples in the composition range near or at the eutectoid, that there is limited exchange spring behavior, even though 


\section{remanence ratios and major loop susceptibilities indicated significant exchange coupling is} present. There is only one existing study of recoil behavior in bulk Co-Pt alloys, by Xiao, et al. $^{55}$. They show that equimolar CoPt can exhibit a high degree of reversibility, but only for a narrow range of process conditions. Exchange-coupling occurs between the $\mathrm{L}_{0}$ and $\mathrm{A} 1$ phases in a partially transformed sample with magnetic properties nominally similar to our peakhardness $\mathrm{Co}_{41.7} \mathrm{Pt}_{58.3}$ sample from Chapter 3, which was annealed at $600{ }^{\circ} \mathrm{C}$ for 96 hours. We have also re-examined this sample, but do not find reversibility to be improved relative to the samples already discussed here.

The lack of reversible exchange-spring behavior in all our materials, despite evidence for exchange-coupling, is puzzling. The degree of reversibility must depend on the dominant mechanism for magnetization reversal. Materials showing exchange-spring behavior in the literature are likely to be dominated by either rotation- or nucleation-based reversal. If the alloys reported here are instead dominated by domain wall pinning, then reversible behavior is not anticipated to be significant, provided these walls move along the rod axis and not orthogonal to it. As it moves along the nanorod axis, a lateral domain wall cutting through the hard and soft phases, illustrated in Figure 4.20, would cause the magnetization in both phases to switch simultaneously, without exhibiting exchange spring behavior, even if the phases are on commensurate lengthscales. Reversal mechanisms are discussed further below.

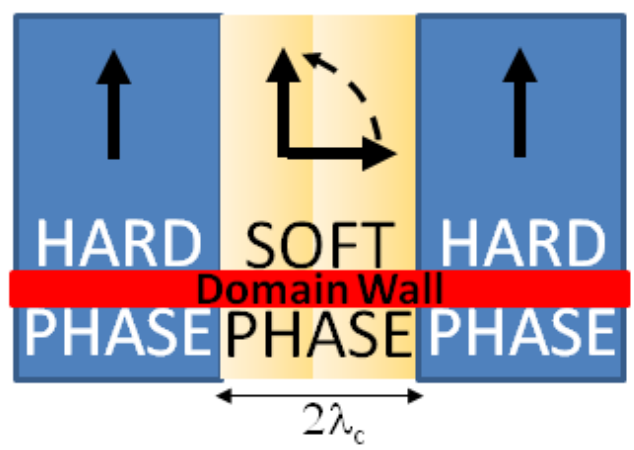

Figure 4.20: Schematic illustration of a lateral domain wall responsible for lack of exchange spring behavior in an exchange coupled nanocomposite.

Additional reasons contributing to lack of exchange spring behavior would be: 
(i) In the chessboard samples discussed here, coupling is between the $\mathrm{L}_{0}$ and $\mathrm{L}_{2}$ phases. Perhaps the $\mathrm{L}_{2}$ may actually not be as magnetically soft as we might expect, given that it is significantly Co-rich with respect to the stoichiometric phase. This would promote a larger tendency towards irreversible rotations in the $\mathrm{L}_{2}$ phase. So in this case, the chessboard microstructure would comprise of hard and medium and not hard and soft materials as was previously thought, which would justify the lack of exchange springiness. However, this fails to explain the lack of reversibility in the $\mathrm{L} 1_{0}+\mathrm{A} 1$ samples of Chapter 3 . In this case, the $\mathrm{A} 1$ is Pt-rich relative to the materials examined by Xiao, et al. ${ }^{55}$, so would not expected to be magnetically harder.

(ii) If the $\mathrm{L1}_{2}$ phase is paramagnetic rather than ferromagnetic at room temperature, then exchange-coupling would not occur. This does not appear feasible based on our preliminary Curie temperature results for the compositions right outside the two phase regime, but requires further assessment using VSM and calorimetry. Again, this would not explain the lack of reversibility in the samples of Chapter 3, unless the A1 phase was also paramagnetic.

(iii) The chessboard produces a unique arrangement of moments, such that each $\mathrm{L}_{2}$ "nanorod" is bordered on adjacent sides by two different $\mathrm{L}_{0}$ c-axis variants (see Figure 1.9), which would create an odd, frustrated magnetization state in the $\mathrm{L}_{2}$ rods. A roughly similar situation would be expected in the $\mathrm{L} 1_{0}+\mathrm{A} 1$ samples of Chapter 3 , and those in Xiao, et al' study ${ }^{55}$. It appears that either their result is inaccurate, or our materials are behaving fundamentally differently.

Kneller and Hawig ${ }^{38}$ have estimated that the optimum magnetic properties of an exchange spring magnet correspond to an underlying microstructure containing $\approx 10 \%$ of the hard phase and $\approx 90$ $\%$ of the soft phase, by volume. Their simple model invoked spherical, hard precipitates arranged on an FCC-like lattice, embedded within a soft matrix, with the concurrent requirement that the particle size and spacing be about equal to the hard-phase domain wall thickness (i.e., the exchange correlation length of the hard phase). The $\mathrm{L}_{0}$ volume fractions obtained in $\mathrm{Co}_{40.2} \mathrm{Pt}_{59.8}$ samples continuously cooled from 750 to $600{ }^{\circ} \mathrm{C} @ 40{ }^{\circ} \mathrm{C} /$ day followed by annealing at $600{ }^{\circ} \mathrm{C}$ were significantly higher than the nominal optimum value. Processing this alloy at temperatures 
lower than $600{ }^{\circ} \mathrm{C}$ may allow for finer lengthscales for chessboards and chessboard colonies. This would also provide greater control on the extent of phase transformation, i.e., in this case, on the volume fraction of the hard phase. Alloys with compositions located on the eutectoid isotherm but more Pt-rich compared to $\mathrm{Co}_{40.2} \mathrm{Pt}_{59.8}$ may allow for lower $\mathrm{L}_{0}$ content, based on the lever rule. Presumably the more Pt-rich $\mathrm{Co}_{38.8} \mathrm{Pt}_{61.2}$ sample might more closely approximate the ideal volume fraction, but much more work is needed to optimize the phase transformation and to characterize the chessboard lengthscales at this stoichiometry. Thus, there is a potential for optimizing the magnetic properties and observing true exchange spring behavior, i.e., reversibility of the magnetization, by suitable changes in either the alloy composition or alloy processing conditions, in order for the microstructure to contain $\approx 10 \%$ of the soft phase and $\approx 90$ $\%$ of the hard phase, by volume.

\subsubsection{Effect of Quench Temperature}

A comparison of $\mathrm{M}-\mathrm{H}$ hysteresis loops and susceptibility behavior derived from them are presented in Figure 4.21 for $\mathrm{Co}_{40.2} \mathrm{Pt}_{59.8}$ samples continuously cooled from $750{ }^{\circ} \mathrm{C}$ at the rate of $40{ }^{\circ} \mathrm{C} /$ day to various temperatures: 690,650 and $600{ }^{\circ} \mathrm{C}$, followed by water quenching. Continuous cooling to $690^{\circ} \mathrm{C}$ resulted in soft single phase behavior, indicated by the single peak in the susceptibility yersus field curve; the slight asymmetry in the low negative field region may indicate the formation of a small amount of hard phase. Thus, the phase transformation is in its initial stages at this stage. The sample obtained upon continuous cooling to $650{ }^{\circ} \mathrm{C}$ is more transformed compared to the one quenched at $690{ }^{\circ} \mathrm{C}$, although less so than the sample quenched at $600{ }^{\circ} \mathrm{C}$. The sample undergoes a small increase magnetic hardening, with a coercivity of 400 Oe, compared to $\sim 100$ Oe in the sample quenched at $690{ }^{\circ} \mathrm{C}$. This susceptibility behavior is consistent with having a fully decoupled soft phase, indicated by the blue arrow and a minor fraction of the harder phase, indicated by the green arrow, which has a range of switching fields. As discussed previously, the susceptibility curves corresponding to the sample continuously cooled to $600{ }^{\circ} \mathrm{C}$ showed two peaks indicating the reversal of the soft and hard phases, respectively marked by a blue arrow, near zero field and a green arrow. A comparison of the relative peak sizes indicated that the major volume fraction still consisted of the uncoupled softer phase. As indicated by its higher coercivity of 1800 Oe, the volume fraction, and likely the 
order parameter, of the hard phase was higher in the sample continuously cooled to $600{ }^{\circ} \mathrm{C}$ than to $650{ }^{\circ} \mathrm{C}$.
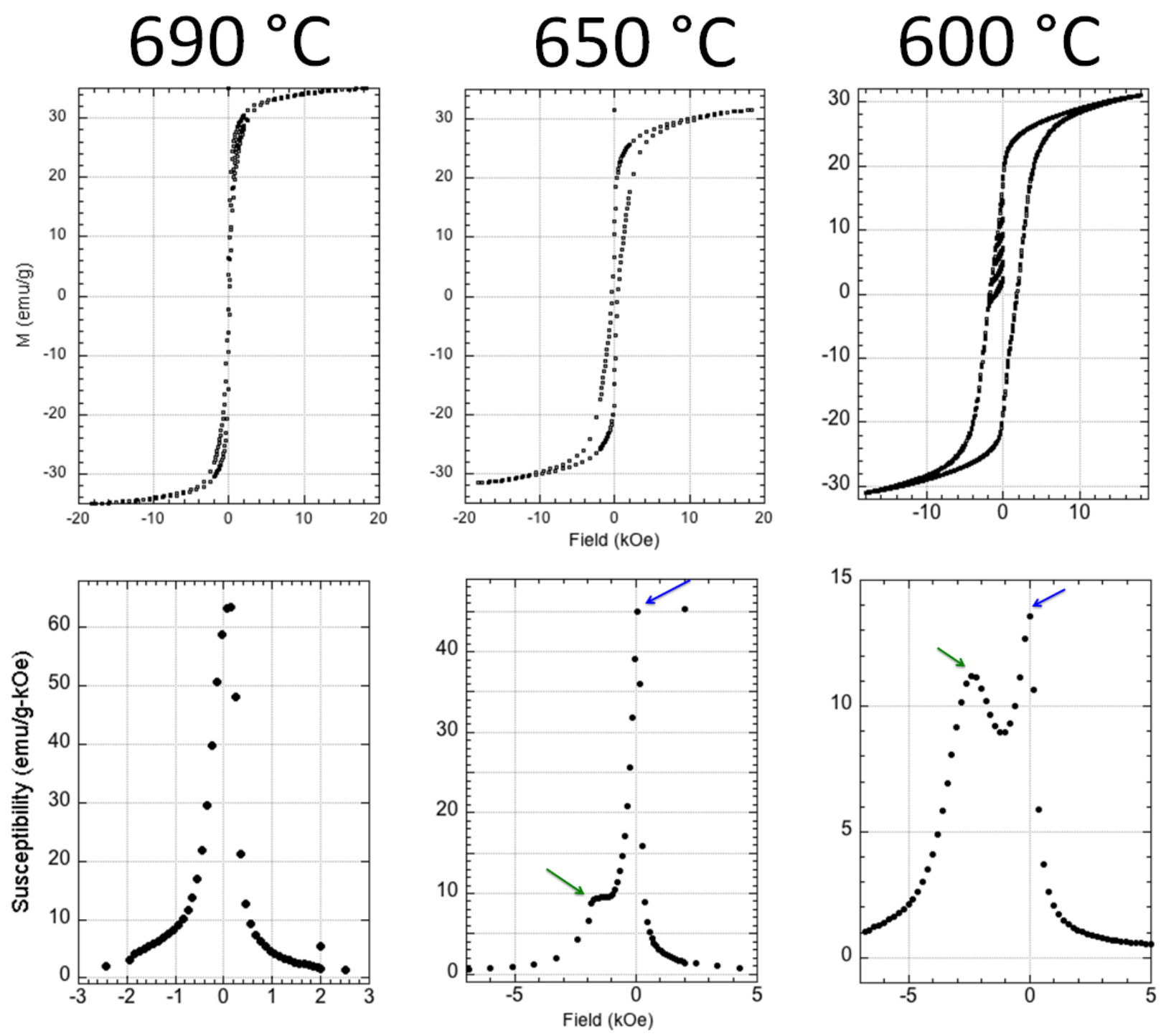

Figure 4.21: $\mathrm{M}-\mathrm{H}$ hysteresis loops and susceptibility versus field curves from $\mathrm{Co}_{40.2} \mathrm{Pt}_{59.8}$ samples continuously cooled at the rate of $40{ }^{\circ} \mathrm{C} /$ day, from 750 to 690,650 and $600{ }^{\circ} \mathrm{C}$ respectively, and then water quenched. 


\subsubsection{Effect of Composition}

As shown in Figure 4.22, identical heat treatment routines produced superior coercivity and remanence ratio in $\mathrm{Co}_{40.2} \mathrm{Pt}_{59.8}$, compared to $\mathrm{Co}_{38.8} \mathrm{Pt}_{61.2}$. This may stem from the lever rule, based on the volume fractions of the soft and hard phases resulting in the two samples after identical heat treatment. The narrow two-phase region means that small changes in the system composition can produce large changes in volume fractions of $\mathrm{L}_{0}$ and $\mathrm{L1}_{2}$. The lever rule predicts a higher volume fraction of the hard $\mathrm{L1}_{0}$ phase in $\mathrm{Co}_{40.2} \mathrm{Pt}_{59.8}$.

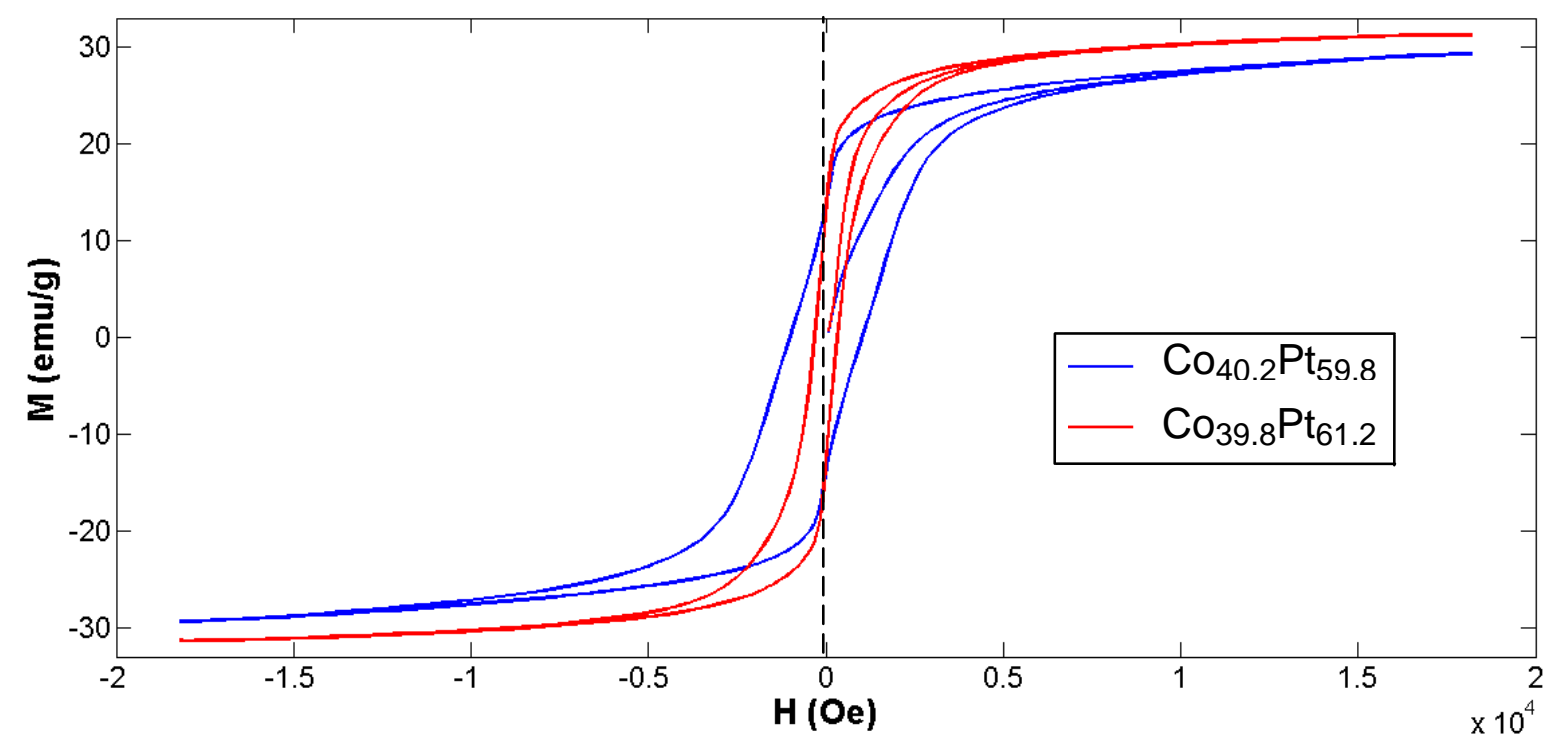

Figure 4.22: $\mathrm{M}(\mathrm{H})$ as a function of composition in samples continuously cooled to $650{ }^{\circ} \mathrm{C}$ and annealed for 3.5 days at that temperature.

Figure 4.23, summarizes the variation in magnetic properties in the continuously cooled samples with annealing time at $650{ }^{\circ} \mathrm{C} . \mathrm{H}_{\mathrm{c}}, \mathrm{BH}_{\max }, \mathrm{M}_{\mathrm{r}}$ and $\mathrm{M}_{\mathrm{r}} / \mathrm{M}_{\mathrm{s}}$ follow the same trend for both compositions, increasing with annealing time, attaining a maximum at 1 week and then decreasing thereafter, as shown in Figure 4.23. $\mathrm{M}_{\mathrm{s}}$ is seen to decrease with annealing time owing to increasing ordering. Figure 4.24 displays the improvement in $\mathrm{H}_{\mathrm{c}}, \mathrm{BH}_{\max }, \mathrm{M}_{\mathrm{r}}$ and $\mathrm{M}_{\mathrm{r}} / \mathrm{M}_{\mathrm{s}}$ with decreasing quench temperature, corresponding to the progress in phase transformation, which is in its initial stages at higher quench temperatures and has occurred to greater degrees at lower quench temperature. Saturation magnetization values of the disordered, single-phase base 
materials are also indicated in the plot showing $M_{s}$ versus quench temperature. The trend in $M_{s}$ for $\mathrm{Co}_{40.2} \mathrm{Pt}_{59.8}$ supports increased ordering upon quenching to lower temperatures, although $\mathrm{Co}_{38.8} \mathrm{Pt}_{61.2}$ does not distinctly follow suit.
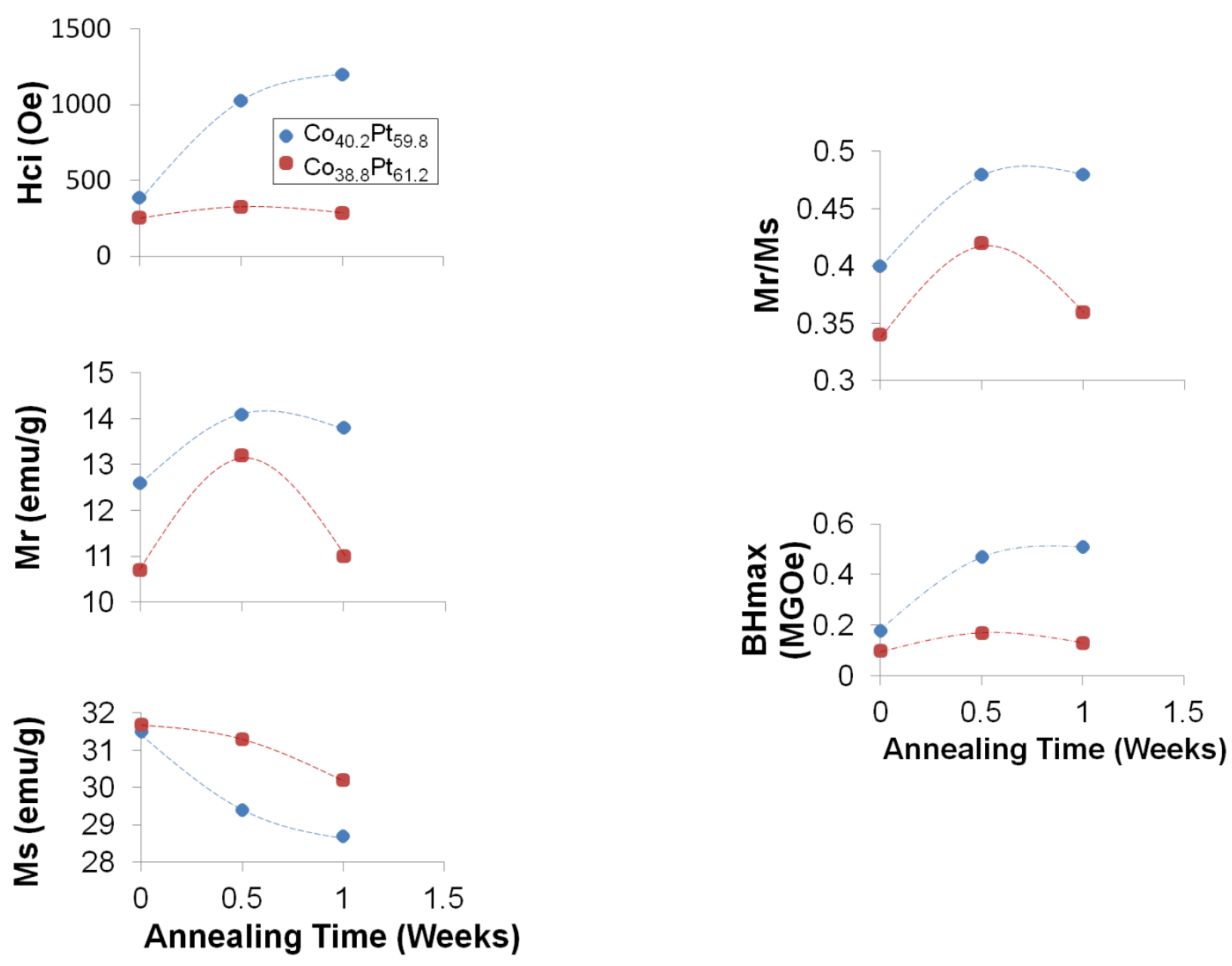

Figure 4.23: Variation of magnetic properties with annealing time in $\mathrm{Co}_{40.2} \mathrm{Pt}_{59.8}$ and $\mathrm{Co}_{38.8} \mathrm{Pt}_{61.2}$ samples upon slow cooling from 750 to $650{ }^{\circ} \mathrm{C}$, with a cooling rate of $40{ }^{\circ} \mathrm{C} /$ day. 

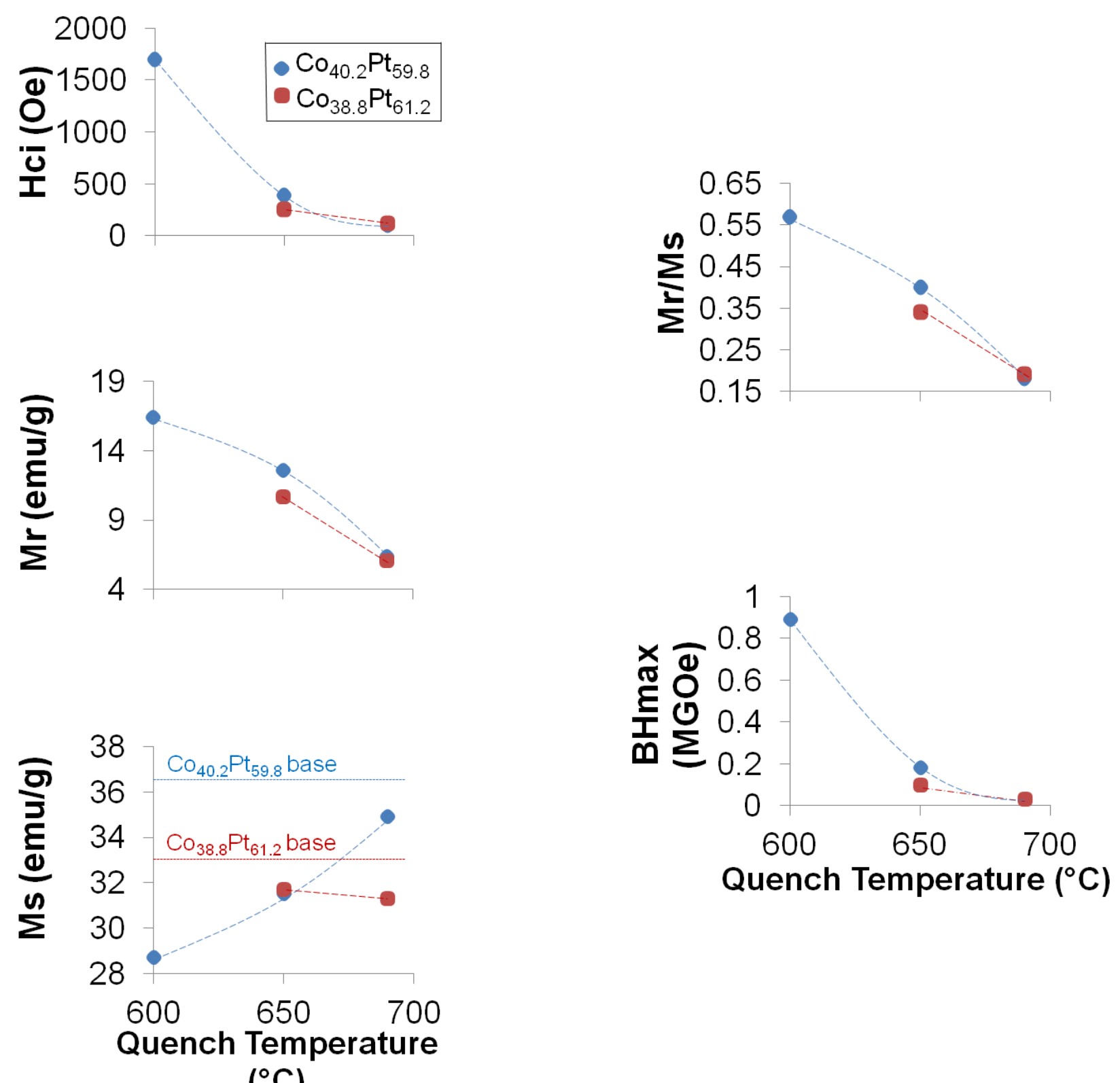

Figure 4.24: Magnetic properties as a function of quench temperature in $\mathrm{Co}_{40.2} \mathrm{Pt}_{59.8}$ and $\mathrm{Co}_{38.8} \mathrm{Pt}_{61.2}$ samples obtained upon cooling from $750{ }^{\circ} \mathrm{C}$ at $40{ }^{\circ} \mathrm{C} /$ day. Saturation magnetization values of the disordered, single-phase base materials are also indicated in the $M_{s}$ versus quench temperature plot.

Both $\mathrm{Co}_{40.2} \mathrm{Pt}_{59.8}$ and $\mathrm{Co}_{38.8} \mathrm{Pt}_{61.2}$ samples showed similar magnetic properties upon water quenching at $650{ }^{\circ} \mathrm{C}$, although the former had slightly higher coercivity, remanence, remanence 
to saturation ratio and $\mathrm{BH}_{\max }$, as shown in Figure 4.23. As seen from Figure 4.24, the coercivity, remanence/saturation were less developed in the $\mathrm{Co}_{40.2} \mathrm{Pt}_{59.8}$ sample water quenched at $650{ }^{\circ} \mathrm{C}$ compared to $600{ }^{\circ} \mathrm{C}$, owing to the lower extent of the phase transformation at the lower undercooling. The changes in microstructural lengthscale and integrity of the chessboard structure alone, as discussed in Section 4.1.3, may be insufficient to explain the changes in observed magnetic properties; changes in the $\mathrm{L} 1_{0}, \mathrm{~L}_{2}$ and retained FCC phases fractions, order parameters of the $\mathrm{L} 1_{0}$ and $\mathrm{L} 1_{2}$ phases and the tetragonality of the $\mathrm{L} 1_{0}$ phase in samples annealed at $600{ }^{\circ} \mathrm{C}$, would contribute significantly to the magnetic properties.

\subsubsection{Effect of Cooling Rate}

The variation of magnetic properties with the rate of gradual cooling is shown in Figure 4.25. The samples were continuously cooled from 750 to $650{ }^{\circ} \mathrm{C} @ 20$ and $40{ }^{\circ} \mathrm{C} / \mathrm{day}$. A lower cooling rate does not lead to an improvement in the coercivity or remanence in $\mathrm{Co}_{40.2} \mathrm{Pt}_{59.8}$. The trend is not as straightforward in $\mathrm{Co}_{38.8} \mathrm{Pt}_{61.2}$, where the coercivity seems to agree with the behavior observed in $\mathrm{Co}_{40.2} \mathrm{Pt}_{59.8}$ but the remanence seems to disagree.

The slower cooling rate would be expected to enhance the overall degree of chemical ordering for a given quench temperature, as well as produce coarser transformation length scales. The fact that $\mathrm{M}_{\mathrm{S}}$ is lower in the $\mathrm{Co}_{40.2} \mathrm{Pt}_{59.8}$ sample water quenched after cooling at $20{ }^{\circ} \mathrm{C} /$ day versus $40{ }^{\circ} \mathrm{C} /$ day is consistent with greater $\mathrm{L} 1_{0}$ ordering. The lower $\mathrm{H}_{\mathrm{c}}$ and $\mathrm{M}_{\mathrm{r}} / \mathrm{M}_{\mathrm{s}}$ are consistent with larger lengthscales for the phases in samples processed at lower cooling rates. Although the higher $\mathrm{M}_{\mathrm{S}}$ in the in $\mathrm{Co}_{38.8} \mathrm{Pt}_{61.2}$ sample processed at $20{ }^{\circ} \mathrm{C} /$ day is slightly odd, the difference between the $\mathrm{M}_{\mathrm{s}}$ obtained from the $20^{\circ} \mathrm{C} /$ day and $40{ }^{\circ} \mathrm{C} /$ day cooling rates is fairly small and may be attributed to the noise of our measurement. Additionally, the lack of a data point at $600{ }^{\circ} \mathrm{C}$ for this sample also makes it difficult to assess trends.

It may be noted that $\mathrm{M}_{\mathrm{s}}$ equals $36.5 \mathrm{emu} / \mathrm{g}$ in the $\mathrm{Co}_{40.2} \mathrm{Pt}_{59.8}$ base material and decays significantly with both quench temperature and extended isothermal annealing, to a minimum of $27.1 \mathrm{emu} / \mathrm{g}$, corresponding to the 2 week annealing treatment at $600{ }^{\circ} \mathrm{C}$. This translates to a $26 \%$ decay in $\mathrm{M}_{\mathrm{s}}$ with annealing/phase transformation. The $\mathrm{Co}_{38.8} \mathrm{Pt}_{61.2}$ base material exhibits an $\mathrm{M}_{\mathrm{s}}$ of 
$33 \mathrm{emu} / \mathrm{g}$, which decays much less with quench temperature and annealing. A minimum of 30 emu/g had been obtained for a sample that had been step cooled from 750 to $600{ }^{\circ} \mathrm{C}$, in steps of $20{ }^{\circ} \mathrm{C}$ every 12 hours and held for weeks, translating to a $10 \%$ decay in $\mathrm{M}_{\mathrm{s}}$. These results are consistent with the larger amount of $\mathrm{Pt}$, and a transformation favoring larger $\mathrm{L}_{2}$ volume fractions in $\mathrm{Co}_{38.8} \mathrm{Pt}_{61.2}$. 

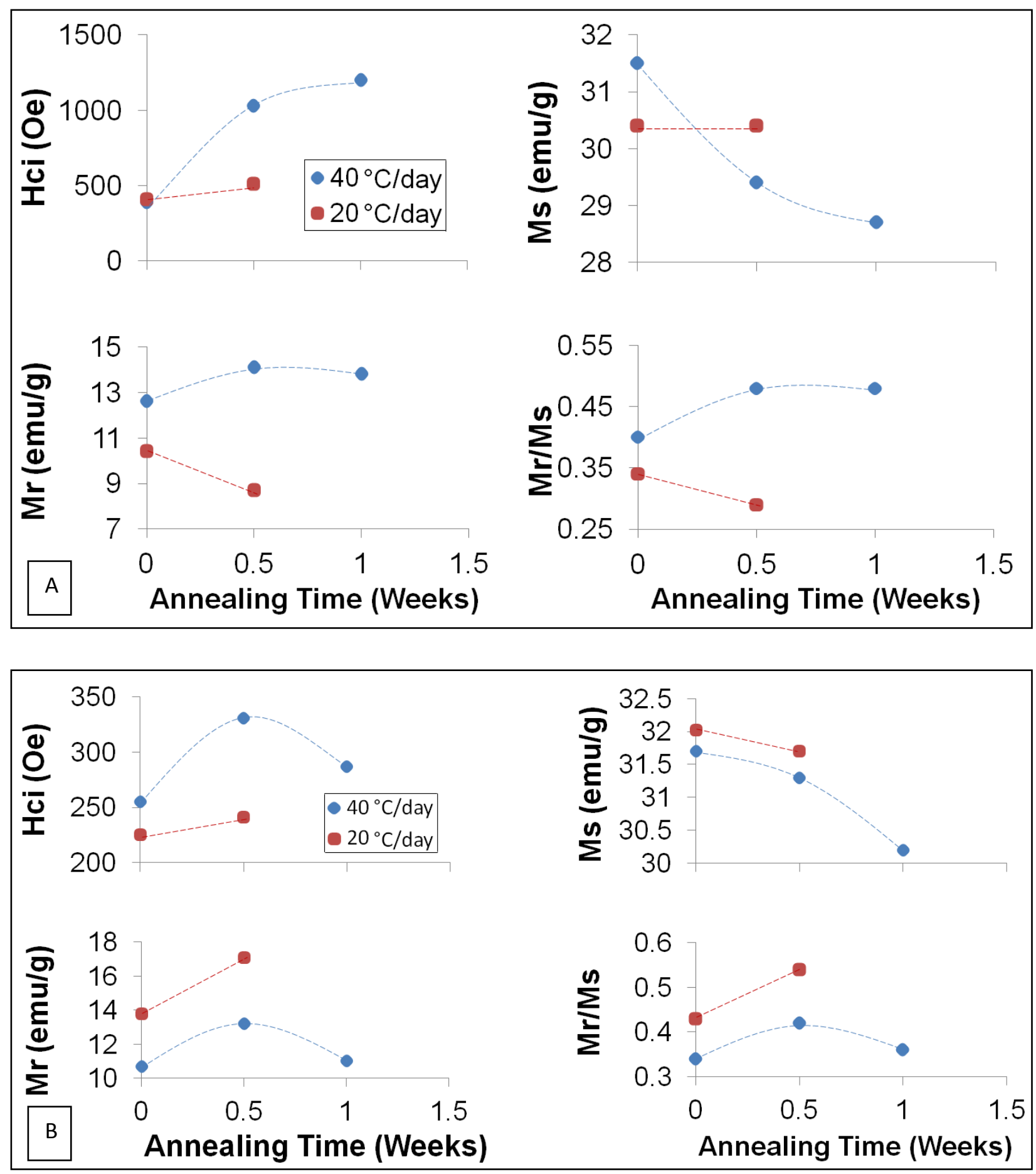

Figure 4.25 (A) Variation of magnetic properties with time and cooling rate in $\mathrm{Co}_{40.2} \mathrm{Pt}_{59.8}$, for annealing at $650{ }^{\circ} \mathrm{C}$. (B) Variation of magnetic properties with time and cooling rate in $\mathrm{Co}_{38.8} \mathrm{Pt}_{61.2}$, for annealing at $650^{\circ} \mathrm{C}$. 


\subsection{Magnetization reversal mechanism}

The shape of the initial magnetization curve is a valuable indicator of the mechanism of magnetization reversal. For instance, domain wall pinning is characterized by an S-shaped initial M-H curve whose first derivative, i.e., susceptibility, exhibits a single peak at an applied field sufficient to enable the domain walls to break free of most pinning sites. The susceptibility decreases at higher fields approaching saturation as the domains have to be rotated against magnetocrystalline anisotropy, i.e., when the easy axis is not parallel to the applied field. The initial magnetization curves of the $\mathrm{Co}_{41.7} \mathrm{Pt}_{58.3}$ alloy samples annealed at $600{ }^{\circ} \mathrm{C}$ for 96 hours shown in Figure 3.5 are consistent with domain wall pinning. The initial magnetization curve for the $\mathrm{Co}_{40.2} \mathrm{Pt}_{59.8}$ chessboard sample shown in Fig 4.13 is also consistent with the domain wall pinning mechanism.

In the case of coherent rotation, the initial magnetization curve is seen to have two distinct regions separated by a kink. The first region is roughly linear with a low-slope, wherein spins rotate against the magnetocrystalline anisotropy but not far enough to switch to the next minimum in the magnetic free energy. As a result, this region is reversible. The kink in the initial magnetization curve occurs when a significant population of moments rotates far enough to overcome the energy barrier between minima and then switch irreversibly. The slope decreases thereafter as the magnetic moments rotate against the magnetocrystalline anisotropy to align with the applied $\mathrm{H}$ field. A typical example of an initial magnetization curve reflecting coherent rotation is shown in Figure 4.26. There is either no pinning, or extremely weak pinning, in a nucleation-controlled magnet, and the existing walls in the demagnetized state move rapidly across the sample with applied magnetizing field resulting in a rapid increase in $\mathrm{M}(\mathrm{H})$. The rate of increase in $\mathrm{M}(\mathrm{H})$ decays as $\mathrm{M}$ approaches $\mathrm{M}_{\mathrm{s}}$, in response to moments as rotating against the magnetocrystalline anisotropy. 


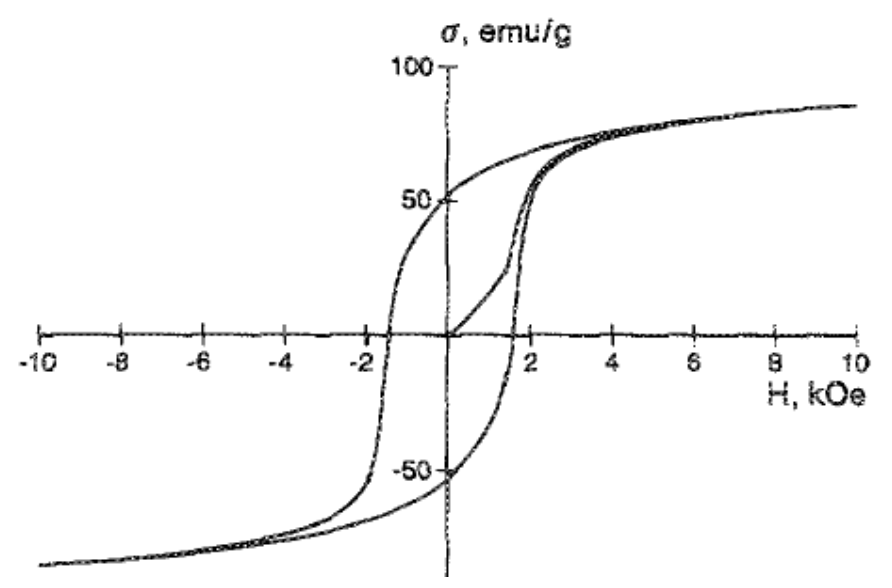

Figure 4.26: An example of an initial magnetization curve distinctly reflecting coherent rotation. 58

A nanochessboard colony is schematically illustrated in Figure 1.1. An enlarged view of a single $\mathrm{L1}_{0}$ nanorod from this colony is shown in Figure 4.27. It is noted that the c axis in each $\mathrm{L} 1_{0}$ nano-rod is orthogonal to the rod axis. Domain walls may be present within the nanorods above a critical rod length. If present, the domain walls would be able to move along the rod axis; their motion may potentially be checked by pinning due to anti phase boundaries (APBs).

Consider a $180^{\circ}$ Bloch wall of thickness $\delta$ passing through a uniform medium under an applied field which encounters a thin perturbed region of thickness $\omega \approx 5 \AA$, with $\omega<<\delta$ such that the perturbed region can lie within the wall. For the domain wall passing through the perturbed region, we may estimate the coercivity due to APB pinning according to Zhang and Soffa ${ }^{22}$ as $\mathrm{H}_{\mathrm{c}}=8 \omega \mathrm{A}_{0} / \pi^{2}\left[\mathrm{~A}^{-1 / 2} \mathrm{~K}^{3 / 2} \mathrm{M}_{\mathrm{s}}^{-1}\right]$. Here, $\mathrm{K}=1.5 * 10^{7}$ ergs/cc is the magnetocrystalline anisotropy constant in the uniform medium and not the perturbed region, $\mathrm{A}$ is the exchange constant in the uniform medium $2.5 * 10^{-6} \mathrm{ergs} / \mathrm{cm}$, while $\mathrm{A}_{0}=0.1$ to 1 is related to the APB density as the fraction of domain wall area containing APBs. $M_{s}=470 \mathrm{emu} / \mathrm{cc}$ for the peak hardness $\mathrm{Co}_{40.2} \mathrm{Pt}_{59.8}$ sample. Using $\mathrm{A}_{0}=1$ gives a theoretical coercivity of 3200 Oe for this peak hardness $\mathrm{Co}_{40.2} \mathrm{Pt}_{59.8}$ sample, while $\mathrm{A}_{0}=0.7$ gives a theoretical coercivity of 2300 , which is equal to the experimentally observed value in this chessboard sample. 


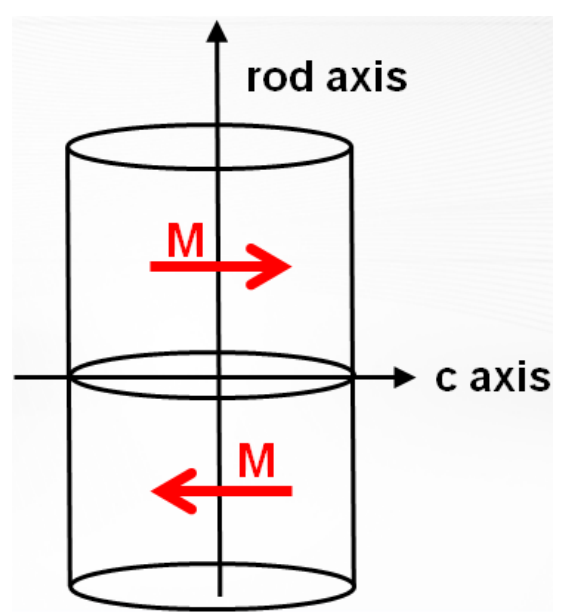

Figure 4.27: Schematic representation of a single $\mathrm{L} 1_{0}$ nanorod within the colony.

The critical rod length for domain wall nucleation in isolated particles, $\mathrm{L}_{\mathrm{c}}=\gamma / \pi \mathrm{M}_{\mathrm{s}}{ }^{2}$, where $\gamma$ is the Bloch wall energy $\approx 28 \mathrm{ergs} / \mathrm{cm}^{2}, \mathrm{M}_{\mathrm{s}}$ is the saturation magnetization, experimentally observed to be $\approx 470 \mathrm{emu} / \mathrm{cc}$ for the $\mathrm{Co}_{40.2} \mathrm{Pt}_{59.8}$ nanochessboard sample obtained upon slow cooling from 750 to $600{ }^{\circ} \mathrm{C} @ 40{ }^{\circ} \mathrm{C} /$ day, followed by annealing at $600{ }^{\circ} \mathrm{C}$ for 1 week. ${ }^{58}$ This yields a value of $\approx 300 \mathrm{~nm}$ for the critical rod length. Furthermore, the critical rod length for domain wall formation is expected to be higher for magnetostatically interacting particles, compared to isolated particles. ${ }^{58}$

It is non-trivial to theoretically rule out the possibility of domains in the complex, heterogeneous chessboard structure. One possibility is that the domain walls may span a colony as a whole rather than being confined to individual rods. The colony could then be considered as a quasispherical "particle", where any domain wall would bridge across all the rods in the colony simultaneously, as shown in Figure 4.28, and reverse the entire chessboard colony as a unit as it moves along the rod axis. This type of behavior has been seen in polytwinned $\mathrm{L}_{0} \mathrm{CoPt}$ by Zhang and Soffa ${ }^{22}$. According to Brown's prediction, the lower limit on the critical single domain size of spherical particles in zero applied field is $2 r_{\mathrm{c} 0}=2\left(3.61 / \mathrm{M}_{\mathrm{s}}\right)(\mathrm{A} / 4 \pi)^{1 / 2}$, all quantities being in cgs units ${ }^{47}$. For $\left(A=2.5 \times 10^{-6} \mathrm{ergs} / \mathrm{cm}\right.$ estimated from $\mathrm{k}_{\mathrm{B}} \mathrm{T}_{\mathrm{c}} / \mathrm{a}$, and $\mathrm{M}_{\mathrm{s}}=470 \mathrm{emu} / \mathrm{cc}$ from our data for) the peak hardness sample, this lower limit is $r_{\mathrm{c} 0} \approx 70 \mathrm{~nm}$; the upper limit which is $15 * \mathrm{r}_{\mathrm{c} 0} \approx 1 \mu \mathrm{m}$. The chessboard colony sizes experimentally determined from TEM 
micrographs are intermediate between the lower and upper bound limits, thus admitting the possibility of such domain walls in the samples.

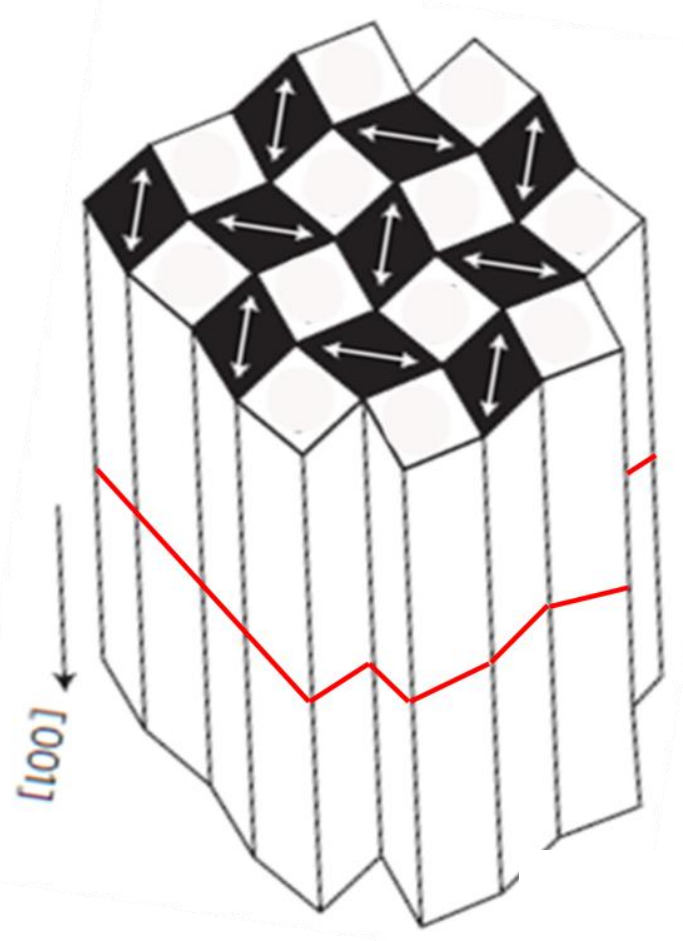

Figure 4.28: Schematic representation of a domain wall (red) bridging across all rods in a chessboard colony.

Magnetization reversal by coherent rotation $^{31}$ would be associated with a coercivity $\mathrm{H}_{\mathrm{ci}}=$ $2 \mathrm{~K} / \mathrm{M}_{\mathrm{s}} \approx 64000 \mathrm{Oe}$, using the above-mentioned values of $\mathrm{K}=1.5 * 10^{7} \mathrm{ergs} / \mathrm{cc}$ and experimental $\mathrm{M}_{\mathrm{s}} \approx 470 \mathrm{emu} / \mathrm{cc}$ for the peak hardness sample. This theoretical value of coercivity is an order of magnitude larger than the experimentally observed value of $\approx 2300$ Oe. Coherent rotation assisted by shape anisotropy has been proposed for particles with anisotropic shapes ${ }^{58}$, which might apply in our case. Accordingly, the shape anisotropy would effectively lower the net anisotropy since $\mathrm{K}_{\text {net }}=\mathrm{K}_{\text {magnetocrystalline }}-\mathrm{K}_{\text {shape, which would allow for a lower theoretical }}$ coercivity, $\mathrm{H}_{\mathrm{c}}=2 \mathrm{~K}_{\mathrm{net}} / \mathrm{M}_{\mathrm{s}}$. For the case of the prolate spheroid ( $\left.\sim \mathrm{rod}\right)$ of semi major axis ' 1 ' and semi-minor axis ' $\mathrm{p}$ ', rod length $2 \mathrm{l}>\operatorname{rod}$ diameter $2 \mathrm{p}, \mathrm{K}_{\text {shape }}=1 / 2 * \Delta \mathrm{N} * \mathrm{M}_{\mathrm{s}}{ }^{2}$, where $\Delta \mathrm{N}$ is the 
difference between the demagnetizing coefficient along major and minor axes ${ }^{31}$. The lower bound of the ratio of the rod length to tile edge, $\sim 1 / p$, would be $120 / 40=3$ in the peak hardness sample. From the work of Stoner and Wohlfarth, ${ }^{48} \Delta \mathrm{N}=4.234$ for $\mathrm{l} / \mathrm{p}=3$ and $\Delta \mathrm{N}=6.283$ for $\mathrm{l} / \mathrm{p}$ $=\infty$. Thus, $\mathrm{K}_{\text {shape }}=1 / 2 * 4.234 *(470)^{2}=4.7 * 10^{5} \mathrm{erg} / \mathrm{cc}$ for $1 / \mathrm{p}=3$ and $\mathrm{K}_{\text {shape }}=1 / 2 * 6.283 *$ $(470)^{2}=6.9 * 10^{5} \mathrm{erg} / \mathrm{cc}$ for $1 / \mathrm{p}=\infty$. In either case, approximating $\mathrm{K}_{\text {shape }}$ to $5 * 10^{5} \mathrm{erg} / \mathrm{cc}, \mathrm{K}_{\text {net }} \approx$ $(150-5) * 10^{5} \mathrm{erg} / \mathrm{cc}$ or $1.45 * 10^{7} \mathrm{erg} / \mathrm{cc}$. This would predict a theoretical coercivity of $\approx 61000$ Oe, which is still much higher than the experimental value. This is consistent with the fact that we observe no obvious indication of coherent rotation in the initial magnetization curves of the chessboard samples.

Our admittedly speculative model for coercivity based on domain wall pinning by APBs is motivated by several factors. First, the initial magnetization curve is consistent with weak pinning. Secondly, analogous behavior has been reported in polytwinned $\mathrm{L}_{0}$ materials such as Fe-Pd where domain walls spanning multiple polytwinned plates have been imaged using Lorentz microscopy, as shown in Figure1.29. These walls were suggested to pin on APBs in the polytwins, whose presence was been established properly there.

Furthermore, the motion of lateral domain walls along the rod axis provides a reasonable explanation for lack of exchange spring behavior in the chessboard samples. Experimental verification of the mechanism of magnetization reversal requires further investigations using Lorentz microscopy. 


\section{B. Summary: Processing-Structure-Property Relationships}

The nanochessboard structure was successfully produced in $\mathrm{Co}_{40.2} \mathrm{Pt}_{59.8}$ and $\mathrm{Co}_{38.8} \mathrm{Pt}_{61.2}$ alloys, and verified using Dark Field TEM. The processing involved slow cooling into the two-phase $\mathrm{L1}_{0}+\mathrm{L1}_{2}$ region, from $750 \mathrm{C}$ to $650{ }^{\circ} \mathrm{C}$ or $600{ }^{\circ} \mathrm{C}$. The samples were either water quenched after the slow cooling or annealed. Isothermal annealing improves chemical ordering, allows for the progress of the phase transformation such that the ordered $\mathrm{L}_{0}$ and $\mathrm{L}_{2}$ phases progress to their equilibrium compositions and volume fractions, and leads to an increase in tetragonality of the $\mathrm{L}_{0}$ phase. Overall, $\mathrm{Co}_{40.2} \mathrm{Pt}_{59.8}$ evidenced superior coercivities and remanence to saturation ratios compared to $\mathrm{Co}_{38.8} \mathrm{Pt}_{61.2}$. Processing the samples at $600{ }^{\circ} \mathrm{C}$ produced superior magnetic properties compared to $650{ }^{\circ} \mathrm{C}$. Among samples clearly evidencing the chessboard structures, slow cooling to $600{ }^{\circ} \mathrm{C}$ followed by annealing at $600{ }^{\circ} \mathrm{C}$ for 1 week resulted in peak hardness of $\sim 2300$ Oe and remanence to saturation ratio of 0 . 66. Further annealing results in overaging, leading to a disintegration of the chessboard structure and a decline in coercivity and exchange coupling. Fully understanding the mechanism of magnetization reversal in the nanochessboards requires further investigations. 


\section{Summary and Future Work}

Off-stoichiometric $\mathrm{L}_{2}$ and $\mathrm{L}_{0}$ phases interleaved on the nanoscale comprise the nanochessboard structure. The individual behavior of these two ordered phases the vicinity of the eutectoid isotherm were first characterized, in order to help better interpret the composite structure in later studies. $\mathrm{Co}_{41.7} \mathrm{Pt}_{58.3}$ showed maximum room temperature coercivity, $4000 \mathrm{Oe}$, as well as the maximum saturation to remanence ratio of 0.8 upon annealing at $600{ }^{\circ} \mathrm{C}$ for 96 hours. This corresponded to early stages of the disorder $\rightarrow$ order transformation with fine ordered $\mathrm{L1}_{0}$ nuclei aligned in and exchange coupled through a disordered FCC matrix producing tweed contrast with a modulation period of $15 \mathrm{~nm}$. In this initial stage, the $\mathrm{L} 1_{0}$ did not evidence significant tetragonality. The magnetization reversal is modeled to occur via by domain wall motion with $\mathrm{L}_{0}$ nanoparticles acting as pinning sites. Even though exchange coupling is quite significant, as suggested by the high remanence-to-saturation ratio (0.80), very little reversibility is evident to the magnetization behavior. Upon extended annealing at $700{ }^{\circ} \mathrm{C}$ for 96 hours, this alloy ordered to predominantly $\mathrm{L}_{0}, \approx 81 \%$ by volume, and $\approx 19 \%$ by volume of $\mathrm{L} 1_{2}$. This suggests that the previously established boundaries of the two-phase coexistence region at that temperature may need revision and that the two phase regions is broader than published. The $\mathrm{Co}_{41.7} \mathrm{Pt}_{58.3}$ alloy's microstructure evolved into the polytwinned structure. The $\mathrm{Pt}$-poor $\mathrm{L}_{2}$ phase was also examined at the composition $\mathrm{Co}_{37.6} \mathrm{Pt}_{62.4}$. This material evolved from $\mathrm{A} 1$ to $\mathrm{L}_{2}$ with no indications of the presence of $\mathrm{L1}_{0}$ at any point during the transformation, which gives confidence in the position of the solvus line between the two-phase region and the $\mathrm{L}_{2}$ single-phase field. A significant finding is that at this stoichiometry, $\mathrm{L}_{2}$ has a greatly elevated Curie temperature relative to $\mathrm{CoPt}_{3}$, so that in the chessboard structure, coupling will between two ferromagnetic phases rather than ferromagnetic-paramagnetic interactions.

The nanochessboard structure was obtained in alloys with compositions on the eutectoid isotherm. We found that it was important to continuously cool these samples through the eutectoid temperature in order to produce the chessboard structure. Magnetic response of Co-Pt nanochessboards was characterized for the first time. This work also demonstrated an approach to quantitatively analyzing the coexisting $\mathrm{A} 1, \mathrm{~L} 1_{0}$ and $\mathrm{L}_{2}$ volume fractions that allowed us to 


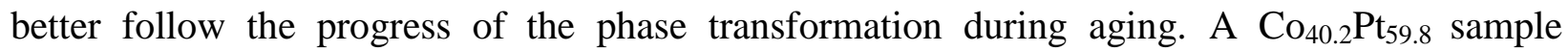
continuously cooled from $750{ }^{\circ} \mathrm{C}$ to $600{ }^{\circ} \mathrm{C}$ at $40{ }^{\circ} \mathrm{C} /$ day, then aged at $600{ }^{\circ} \mathrm{C}$ for 1 week followed subsequently by water quenching produced a chessboard structure corresponding to maximum coercivity, $2300 \mathrm{Oe}$, as well as maximum remanence to saturation ratio, 0.66 , indicative of exchange coupling. At this stage the sample contained exchange-coupled hard $\mathrm{L}_{0}$ and soft $\mathrm{L}_{2}$; as well as uncoupled FCC phase. Again, inspite of evidence for exchange coupling, the chessboards showed lack of expected. exchange spring behavior which may result from the motion of lateral domain walls cutting across rods. Longer annealing durations, such as 2 weeks, led to an overaged microstructure reminiscent of that predicted by Kneller and Hawig [see Ref. 38].

Some avenues for further investigation are as follows:

- Experimentally verify mechanism of magnetization reversal in nanochessboard using Lorentz microscopy.

- Develop processing techniques inhibiting lower energy mechanisms of magnetization reversal. For instance, only APBs are available to pin the domain walls as they move along the rod length, at present; introducing additional, stronger pinning sites would be desirable.

- Confirm eutectoid temperature from the DSC investigation of additional chessboard samples.

- Optimize magnetic properties and control tile size in chessboard samples. Conducting isothermal annealing at even lower temperatures, say $550{ }^{\circ} \mathrm{C}$, may be useful in this regard.

- Figure 5.1 shows a comparison of $\mathrm{M}-\mathrm{H}$ and susceptibility curves for $\mathrm{Co}_{40.2} \mathrm{Pt}_{59.8}$ samples slow cooled from 750 to $650{ }^{\circ} \mathrm{C}$ at (A) $40{ }^{\circ} \mathrm{C} /$ day and (B) $20^{\circ} \mathrm{C} /$ day, followed by water quenching. Both cooling rates resulted in near identical behavior, for e.g., two peaks in their susceptibility curves (although the peaks need to be deconvoluted to be clearly seen in case of the lower cooling rate). There appeared to be a slightly larger volume fraction of the hard phase and a slightly lower saturation magnetization in the sample cooled at 20 ${ }^{\circ} \mathrm{C} /$ day. The absolute magnitudes of the susceptibilities were a bit smaller in this case, suggesting a greater distribution of pinning sites. While this is consistent with a slightly 
more progressed phase transformation in case of slower cooling, it does not reflect significant overaging. This suggests that doubling the time in the upper temperature range $\left(750{ }^{\circ} \mathrm{C}-650^{\circ} \mathrm{C}\right)$ does not promote much additional phase transformation, and that large undercooling is actually quite important to drive the transformation. This requires further investigation and an additional comparison with a high cooling rate, such as $100{ }^{\circ} \mathrm{C} / \mathrm{day}$ would prove useful in determining the impact of the slow cooling step in the processing technique. A comparison of microstructures of this and existing samples would enable a correlation between the microstructure and the cooling rate.
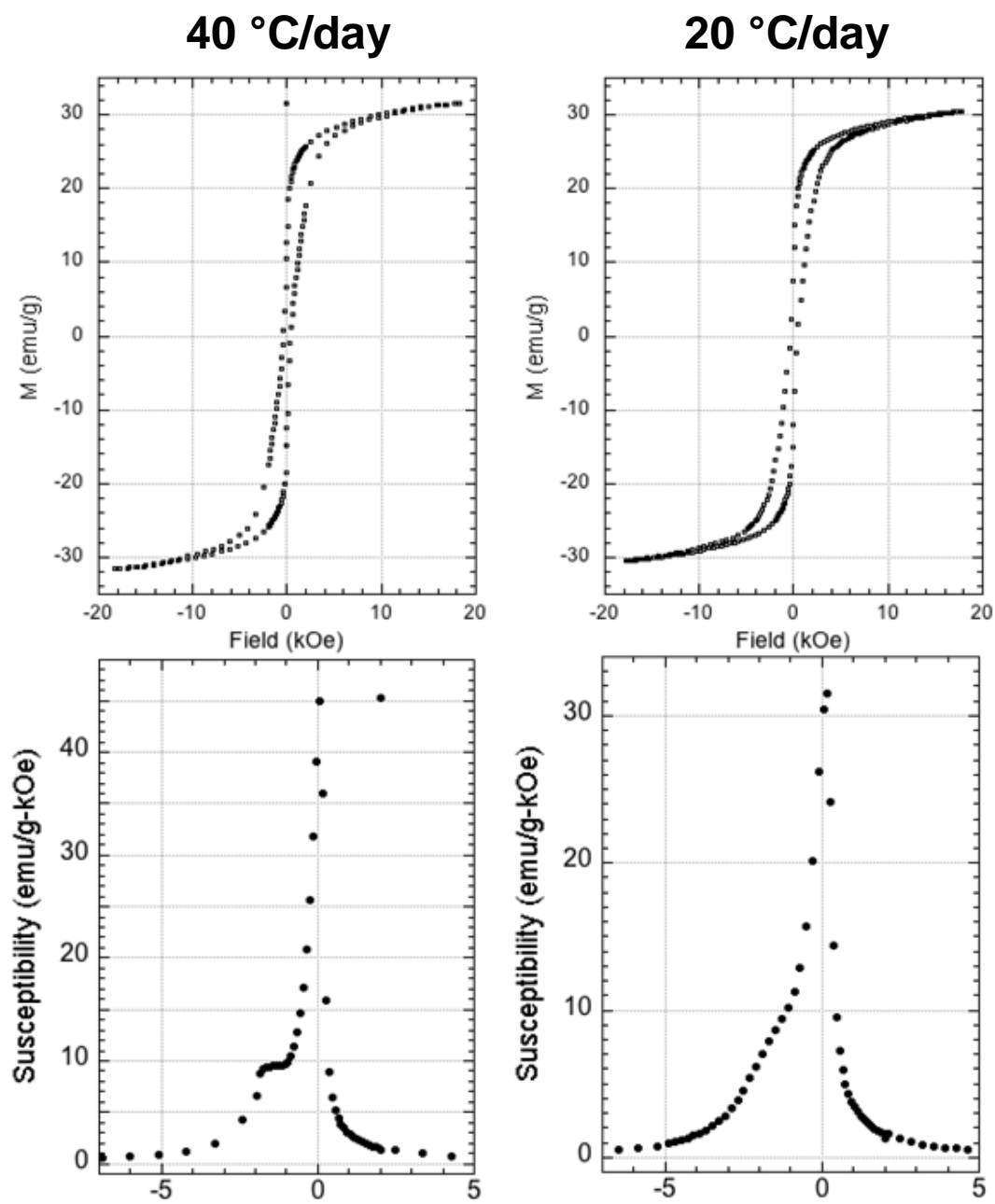

Figure 5.1: $M$ versus $H$ and susceptibility $(\mathrm{dM} / \mathrm{dH}$ versus $\mathrm{H})$ curves for $\mathrm{Co}_{40.2} \mathrm{Pt}_{59.8}$ samples slow cooled from 750 to $650{ }^{\circ} \mathrm{C}$, as a function of cooling rate. 


\section{References:}

1. Leroux, C.; Loiseau, A.; Broddin, D.; Vantendeloo, G. Philosophical Magazine B-Physics of Condensed Matter Statistical Mechanics Electronic Optical and Magnetic Properties 1991, 64 (1), 57.

2. Le Bouar, Y.; Loiseau, A.; Khachaturyan, A. G. Acta Materialia 1998, 46 (8), 2777.

3. Ni, Y.; Khachaturyan, A. G. Nature Materials 2009, 8 (5).

4. Gutfleisch, O.; Lyubina, J.; Muller, K. H.; Schultz, L. Advanced Engineering Materials 2005, 7 (4), 208.

5. Mehaddene, T.; Kentzinger, E.; Hennion, B.; Tanaka, K.; Numakura, H.; Marty, A.; Parasote, V.; Cadeville, M. C.; Zemirli, M.; Pierron-Bohnes, V. Physical Review B 2004, 69 (2), 294.

6. Hadjipanayis, G. C. Journal of Magnetism and Magnetic Materials 1999, 200 (1-3), 373.

7. Hanitsch, R., Rare-Earth Iron Permanent Magnets. Oxford, UK, 1996.

8. Harris, I. R., Rare-Earth Iron Permanent Magnets. 1996.

9. Klemmer, T.; Hoydick, D.; Okumura, H.; Zhang, B.; Soffa, W. A. Scripta Metallurgica Et Materialia 1995, 33 (10-11), 1793.

10. Cantando, E. D. Combined Reactions Thermomechanical Processing Applied To Ferromagnetic FePd Binary Allys`. University of Virginia, 2011.

11. Hansen, M.; Shunk, F. A., Constitution of binary alloys. McGraw Hill: New York, 1958.

12. Polatoglou, H. M. Materials Science and Engineering B-Solid State Materials for Advanced Technology 1996, 37 (1-3), 177.

13. Alloy Physics: A Comprehensive Reference. John Wiley \& Sons: 2008.

14. Laughlin, D. E.; Srinivasan, K.; Tanase, M.; Wang, L. Scripta Materialia 2005, 53 (4), 383.

15. Darling, A. S. Platinum Metals Review 1963, 7 (3), 96.

16. Porter, D. A.; Easterling, K. E., Phase Transformations in Metals and Alloys. Second ed.

17. Cayron C. TEM study of interfacial reactions and precipitation mechanisms in $\mathrm{Al} 2 \mathrm{O} 3$ short fiber or high volume fraction $\mathrm{SiC}$ particle reinforced $\mathrm{Al}-4 \mathrm{Cu}-1 \mathrm{Mg}-0.5 \mathrm{Ag}$ squeeze-cast composites. École Polytechnique Fédérale de Lausanne, 2000..

18. Sanati, M.; Albers, R. C.; Lookman, T.; Saxena, A. Physical Review B 2013, 88 (2), 024110.

19. Chen, H.; Cohen, J. B. Acta Metallurgica 1979, 27 (4), 603.

20. Cullity, B., Elements of X-ray Diffraction. 1956.

21. Vlasova, N. I.; Kandaurova, G. S.; Shchegoleva, N. N., The Physics of Metals and Metallography 2000, $90(3), 239$.

22. Zhang, B.; Soffa, W. A. Physica Status Solidi a-Applied Research 1992, 131 (2), 707.

23. Zhang, B.; Lelovic, M.; Soffa, W. A. Scripta Metallurgica Et Materialia 1991, 25 (7), 1577. 
24. Khachaturyan, A. G., Theory of Structural Transformations in Solids. 1983.

25. Robertson, I. M.; Wayman, C. M. Philosophical Magazine a-Physics of Condensed Matter Structure Defects and Mechanical Properties 1983, 48 (3), 421.

26. Vlasova, E. N.; Gorbunov, V. L.; Komarova, I. A.; Sapozhkova, T. P., Fizika Metallov i Metallo edenie 1972, 34.

27. Vlasova, N. I.; Kandaurova, G. S.; Shchegoleva, N. N. Journal of Magnetism and Magnetic Materials 2000, $222(1-2), 138$.

28. Ni, Y.; Khachaturyan, A. G. Acta Materialia 2008, 56 (16), 4498.

29. Soffa, W. A.; Cantando, E. D.; Shugart, K. N., Solid State Phenomena 2011, 172-174, 344.

30. Frey, N. A.; Peng, S.; Cheng, K.; Sun, S. Chemical Society Reviews 2009, 38 (9), 2532.

31. Cullity, B. D.; C.D., G., Introduction to Magnetic Materials. Second ed.; 2009.

32. http://ocw.mit.edu/courses/materials-science-and-engineering/3-024-electronic-optical-and-magneticproperties-of-materials-spring-2013/lecture-notes/MIT3_024S13_2012lec26.pdf.

33. Spaldin, N., Magnetic Materials Fundamentals and Device Applications. Cambridge University Press: 2003.

34. Zhang, B.; Soffa, W. A. Scripta Metallurgica Et Materialia 1994, 30 (6), 683.

35. Penisson, J. M.; Bourret, A.; Eurin, P. Acta Metallurgica 1971, 19 (11), 1195.

36. The Ising Model of Ferromagnetism. http://www.physics.buffalo.edu/phy411-506/topic2/topic2lec3.pdf.

37. Liu, F.; Hou, Y.; Gao, S. Chemical Society Reviews 2014, 43 (23), 8098.

38. Kneller, E. F.; Hawig, R. Ieee Transactions on Magnetics 1991, 27 (4), 3588.

39. Skomski, R.; Coey, J. M. D. Physical Review B 1993, 48 (21), 15812.

40. Hou, X.; Jones, B. T., Inductively Coupled Plasma - Optical Emission Spectroscopy. In Encyclopedia of Analytical Chemistry, Meyers, R. A., Ed. 2000; 9468.

41. Kundu, S.; Nath, T. K. http://arxiv.org/ftp/arxiv/papers/1105/1105.6313.pdf;

42. (USCB) VSM Option User's Manual

43. Niazi, A.; Poddar, P.; Rastogi, A. K., Current Science 2000, 79 (1), 99.

44. Warren, B. E., X-ray diffraction, 1990.

45. Hadjipanayis, G.; Gaunt, P. Journal of Applied Physics 1979, 50 (3), 2358.

46. Xiao, Q. F.; Bruck, E.; Zhang, Z. D.; de Boer, F. R.; Buschow, K. H. J. Journal of Alloys and Compounds 2004, 364 (1-2), 64.

47. Brown, W. F., Jr., Journal of Applied Physics 1968, 39 (2), 993.

48. Stoner, E. C.; Wohlfarth, E. P., Philosophical Transactions of the Royal Society A 1948, 240, 599. 
49. Zhou, J.; Kashyap, A.; Liu, Y.; Skomski, R.; Sellmyer, D. J. Ieee Transactions on Magnetics 2004, 40 (4), 2940.

50. Craik, D. J., Ferromagnetism and Ferromagnetic Domains, Series of Monographs on Topics on Solid State Physics. North Holland: Amsterdam, 1965; Vol. 4.

51. Zhou, J.; Skomski, R.; Sorge, K. D.; Sellmyer, D. J. Scripta Materialia 2005, 53 (4), 453.

52. Gaunt, P. Canadian Journal of Physics 1987, 65 (10), 1194.

53. Shima, H.; Oikawa, K.; Fujita, A.; Fukamichi, K.; Ishida, K.; Nakamura, S.; Nojima, T. Journal of Magnetism and Magnetic Materials 2005, 290, 566.

54. Mitsek, A. I.; Semyannikov, S. S., Soviet Phys. - Solid State 1969, 11, 899.

55. Xiao, Q. F.; Bruck, E.; Zhang, Z. D.; de Boer, F. R.; Buschow, K. H. J. Journal of Applied Physics 2002, 91 (1), 304.

56. Harland, C. L.; Lewis, L. H.; Chen, Z.; Ma, B. M. Journal of Magnetism and Magnetic Materials 2004, $271(1), 53$.

57. Feutrill, E. H.; McCormick, P. G.; Street, R. Journal of Physics D-Applied Physics 1996, 29 (9), 2320.

58. Zeltser, A. M.; Soffa, W. A. Journal of Applied Physics 1990, 67 (11), 6958. 


\section{Appendix 1: ICP Error Analysis}

The statistical error associated with composition determination using ICP-OES may be estimated as follows:

The ICP-OES outputs a 'ppm' value for each element present, which is proportional to the total mass of the element in solution. Hence,

$<\mathrm{Co}>=\mathrm{k} * \mathrm{j}_{\mathrm{Co}} \quad$...(Eq. A1.1)

$\langle\mathrm{Pt}\rangle=\mathrm{k} * \mathrm{j}_{\mathrm{Pt}} \quad \ldots($ Eq. A1.2)

where $\langle\mathrm{Co}\rangle,\langle\mathrm{Pt}\rangle$ represent the ppm values, $\mathrm{k}$ is a proportionality constant and $\mathrm{j}_{\mathrm{Co}}, \mathrm{j}_{\mathrm{Pt}}$ represent the total mass in solution of Co and Pt respectively.

Let $\mathrm{n}_{\mathrm{Co}}, \mathrm{n}_{\mathrm{Pt}}$ represent the number of $\mathrm{Co}$ and $\mathrm{Pt}$ atoms in solution, respectively. $\xi_{\mathrm{Co}}$, $\xi_{\mathrm{Pt}}$ are the corresponding atomic weights and $\mathrm{N}_{\mathrm{A}}$ is Avogadro's number. Then,

$\mathrm{n}_{\mathrm{Co}}=\mathrm{j}_{\mathrm{Co}} *\left(\mathrm{~N}_{\mathrm{A}} / \xi_{\mathrm{Co}}\right)$

$\mathrm{n}_{\mathrm{Pt}}=\mathrm{j}_{\mathrm{Pt}} *\left(\mathrm{~N}_{\mathrm{A}} / \xi_{\mathrm{Pt}}\right)$

Let $\mathrm{C}_{\mathrm{Co}}$ denote the atom $\% \mathrm{Co}$ in the alloy.

Then, $\mathrm{C}_{\mathrm{Co}}=100 * \mathrm{n}_{\mathrm{Co}} /\left(\mathrm{n}_{\mathrm{Co}}+\mathrm{n}_{\mathrm{Pt}}\right)=100 * \mathrm{j}_{\mathrm{Co}} / \xi_{\mathrm{Co}} /\left[\left(\mathrm{j}_{\mathrm{Co}} / \xi_{\mathrm{Co}}\right)+\left(\mathrm{j}_{\mathrm{Pt}} / \xi_{\mathrm{Pt}}\right)\right]=$ $100 *$ $\mathrm{j}_{\mathrm{Co}} /\left[\mathrm{j}_{\mathrm{Co}}+\mathrm{j}_{\mathrm{Pt}}\left(\xi_{\mathrm{Co}} / \xi_{\mathrm{Pt}}\right)\right]$

$$
\Rightarrow \mathrm{C}_{\mathrm{Co}}=100 *<\mathrm{Co}>/(<\mathrm{Co}>+\mathrm{L}<\mathrm{Pt}>) \quad \ldots(\mathrm{Eq} . \mathrm{A} 1.5)
$$

where $\mathrm{L}=\xi_{\mathrm{Co} /} \xi_{\mathrm{Pt}}=0.3$.

Differentiating Eq. A1.5, we get:

$\mathrm{dC}_{\mathrm{Co}}=100 * \mathrm{~L} *[<\mathrm{Pt}>\mathrm{d}<\mathrm{Co}>-\langle\mathrm{Co}>\mathrm{d}<\mathrm{Pt}\rangle] /[\langle\mathrm{Co}\rangle+(\mathrm{L} *\langle\mathrm{Pt}\rangle)]^{2}$

where $\mathrm{dC}_{\mathrm{Co}}$ is the uncertainity in the atom $\%$ of Co determined by ICP-OES while $\mathrm{d}<\mathrm{Co}>$ and $\mathrm{d}\langle\mathrm{Pt}\rangle$ are the standard deviations in the concentration ( $\mathrm{ppm})$ of Co and $\mathrm{Pt}$ respectively. 
Substituting the experimental values for $\langle\mathrm{Co}\rangle, \mathrm{d}\langle\mathrm{Co}\rangle,\langle\mathrm{Pt}\rangle$ and $\mathrm{d}\langle\mathrm{Pt}\rangle$ for the $\mathrm{Co}_{41.7} \mathrm{Pt}_{58.3}$ alloy in Eq. A1.6, we get:

$$
\begin{aligned}
& \left|\mathrm{dC}_{\mathrm{Co}}\right|=\left|100 * 0.3 *[(67.33 * 0.13)-(14.61 * 0.63)] /[14.61+(0.3 * 67.33)]^{2}\right| \\
& \Rightarrow \mathrm{dC}_{\mathrm{Co}}= \pm 0.01
\end{aligned}
$$

A more conservative estimate of the error would be:

$\mathbf{d C}_{\mathbf{C o}}=100 * \mathrm{~A} *[\langle\mathrm{Pt}\rangle \mathrm{d}\langle\mathrm{Co}\rangle+\langle\mathrm{Co}\rangle \mathrm{d}\langle\mathrm{Pt}\rangle] / 2[\langle\mathrm{Co}\rangle+(\mathrm{A} *\langle\mathrm{Pt}\rangle)]^{2} \quad \ldots($ Eq. A 1.7$)$

which would give an error of $\pm 0.2 \%$. 


\section{Appendix 2: XRD}

Precautions and practices recommended for obtaining better XRD results are outlined below.

\section{Silicon Standard:}

A silicon standard from the National Institute of Standards and Technology can be tested prior to running a series of scans, starting at a $2 \theta=20^{\circ}$ and using the desired divergence angle $\left(1 / 2^{\circ}\right)$. The peaks from this standard are compared to those given by NIST. The diffractometer and data acquisition program are taken to be operating accurately if the actual and theoretical peak positions are within $0.01^{\circ}$ of each other.

\section{Sample Mounting:}

A small piece of putty is first placed in the sample holder. An amorphous glass piece is placed above the putty, followed by the sample on top. The sample is fixed to the glass piece using double sided tape. A flat plastic sheet (available in the XRD lab) is then placed over the sample and pressed down until the top surface of the sample is level with the top of the holder. This plastic sheet has a circle with the diameter of the sample holder marked on it, in addition to mutually perpendicular lines passing through the center of the circle, which are used to position the sample in the approximate center of the sample holder.

\section{Sample Preparation:}

The samples should not undergo surface treatments after thermal processing and prior to XRD analysis. Grinding, for instance, can cause deformation and lead to changes in the crystal structure at the surface of the material. If the sample needs to be ground/polished for other methods of analysis, such as TEM, or even to punch out a disc for VSM, a portion of the original sample should be cut using a cut-off saw and reserved for XRD scans.

\section{Programs:}

The input parameters for data acquisition are discussed below:

- Divergence angle: A divergence angle of $12^{\circ}$ provides a good signal to noise ratio. 
- Step size: For rough scans over a large $2 \theta$ range, a step size of $\sim 0.01$ provides sufficient resolution for both qualitative and quantitative analysis in a reasonably low time duration. For high resolution scans, a smaller step size of $\sim 0.008$ is used, allowing for the acquisition of a larger number of data points. Since the required acquisition time is higher, high resolution scans are restricted to elucidating the structure of specific peaks, for example the $(200) /(002)$ peak in the Co-P alloys.

- Time per step: This variable controls the amount of time spent in acquiring each data point, and consequently the number of counts received by the detector per data point. For rough scans, a time per step of 20 s is used. A time per step of 180 s provides adequate detail in high resolution scans.

The data acquired from the scan should be saved as a .xy file for analysis.

\section{$\underline{\text { Data Analysis }}$}

Peak fitting for XRD data analysis may be conducted using the free software, Magic Plot as follows:

- Open Magic Plot.exe. Drag and drop the .xy file containing the XRD data from a particular scan; click import.

- Select the A and B columns, right click; select create fit plot and then, line.

- A window containing the plot will open up. Click on the 'Fit Curves' tab in this window, then on 'Add' and select 'Line'.

- Position this line to be the baseline for the plot and check off the box below 'Bas..'.

- Click on the 'Fit Interval' tab and enter the appropriate interval based on the peak that needs to be fit. Zoom in on this peak.

- Click on 'Add' and select 'Lorentzian (or Gaussian) '. Add more Lorentzian (or Gaussian) curves if the peak appears to or is believed to contain those many convoluted or readily apparent peaks. Move the Lorentzian (or Gaussian) curves to approximately coincide with these peaks. Now click on 'Fit by Sum'.

An example of the 2-peak (001) Gaussian fit and 4-peak $\{200\} /(002)$ Gaussian fits is shown in Figure A2.1 (a) and (b), respectively. 

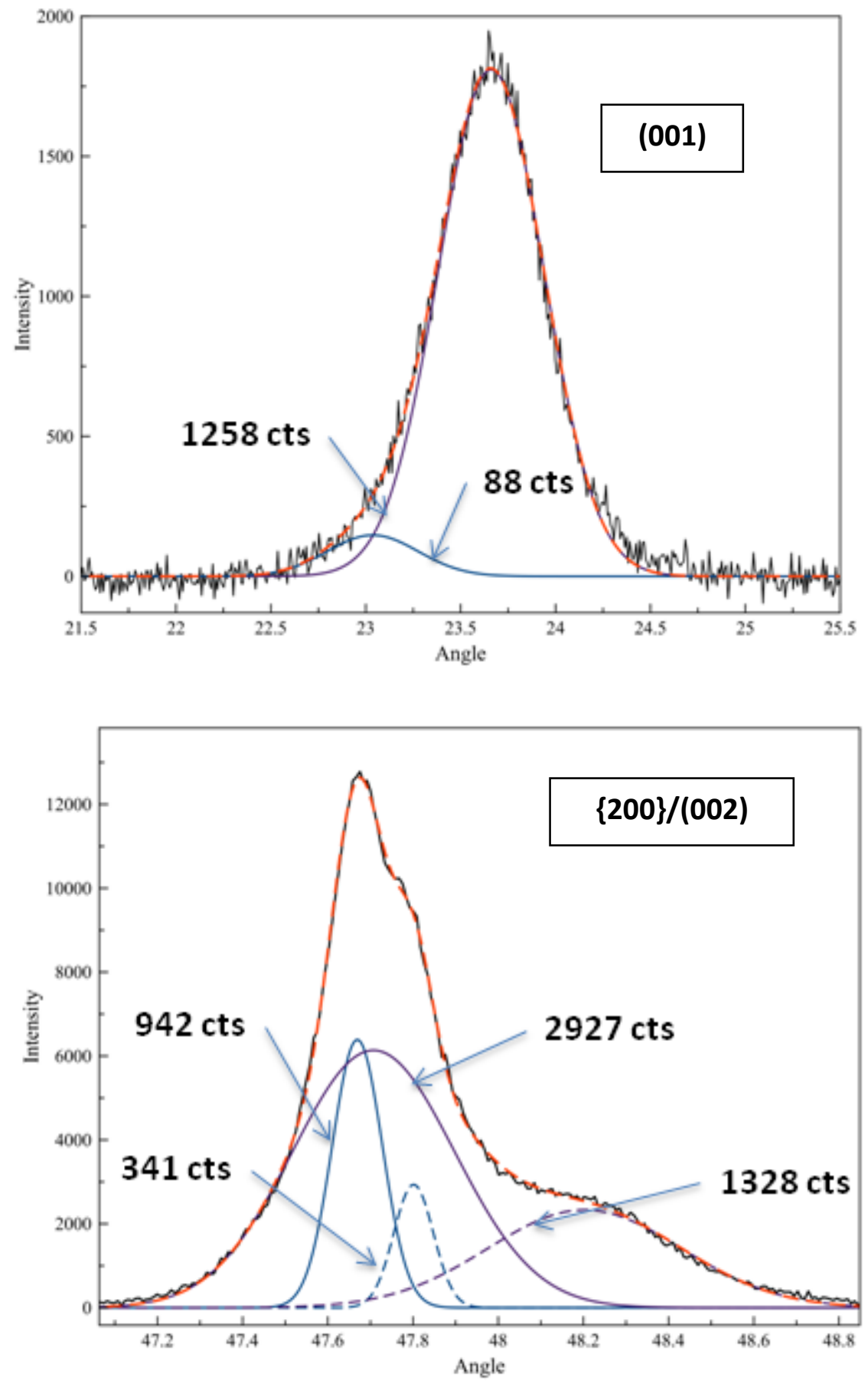

Figure A2.1: (a) 2 peak Gaussian fit to (001) reflection where the peak at lower $2 \theta$ corresponds to $\mathrm{L}_{2}$ and higher $2 \theta$ to $\mathrm{L} 1_{0}$; (b) 4 peak Gaussian fit to the $\{200\} / 002$ reflections where the two narrow peaks at lower $2 \theta$ correspond to $(200)_{\mathrm{K} \alpha 1}$ (solid curve) and to $(200)_{\mathrm{K} \alpha 2}$ (dashed curve) from the soft phases: $\mathrm{A} 1+\mathrm{L1}_{2}$; the broad peak at lower $2 \theta$ corresponds to (200) from $\mathrm{L}_{0}$ and the broad peak at higher $2 \theta$ corresponds to (002) from $\mathrm{L1}_{0}$. 


\section{Appendix 3: TEM sample preparation}

Additional details that can help in TEM sample preparation are given below.

- Grind samples to 100 microns before punching out discs.

- The Gatan Disc Grinder system facilitates uniform grinding of the discs. Crystal Bond can be used to make the disc adhere to the polishing stub.

- Take off 25 microns from both sides of the disc, i.e., a total of 50 microns, using 600, 800 and 1200 grit abrasive discs, manually. Sonicate the stub and the glued on sample in an acetone bath to quickly detach the sample. Clean the sample with ethanol after it is detached.

- For dimpling, the sample may be attached to the dimpling stub using Crystal Bond. The step involving dimpling proved to be the most challenging, owing to difficulty in accurately measuring the dimple thickness using the dimpler. The Hirox optical microscope may be used to determine the thickness of the dimple from the difference in $\mathrm{z}$ associated with changing the focus from the outer rim of the sample to the dimple.

- Prior to ion milling, it is important to ensure that the ion beams are well aligned, using the fluorescent beam alignment screen. The gun angles must be at 10 degrees, top; the ion beams appear as two blue lines on the screen if both guns are turned on. The visibility of the beams will be compromised if the room is brightly lit during this adjustment. Turn off the gas flow switch for one of the guns and work with the remaining beam. Adjust the corresponding gas flow knob to center and maximize the intensity of the beam. Repeat process with the other beam.

- Ensure that the sample rotates during ion milling, in order to prevent artifacts. 


\section{Appendix 4: Error estimation}

Volume fraction error may be determined as follows:

1. Open the high resolution x-ray data for a peak in Magic plot and determine the $2 \theta$ range of the noise.

2. Open the intensity versus noise data in excel: determine the average and standard deviation.

3. Calculate the volume fractions as a function of the choice of baseline, e.g., baseline may be positioned at average noise, average noise $+0.25 *$ standard deviation, average noise $0.25^{*}$ standard deviation, average noise $+0.5 *$ standard deviation, average noise $0.5 *$ standard deviation etc.

4. Now compare these results with the volume fraction obtained from fitting the baseline visually.

Error in order parameter also depend on baseline fitting. A simple error estimate is based on the assumption that the chessboard sample annealed for 1 week giving an order parameter of 0.86 is fully ordered. The composition of this alloy is $\mathrm{Co}_{40.2} \mathrm{Pt}_{59.8}$ and the maximum order parameter associated with it would be $2 * 0.402=0.80$. Thus, we estimate that the order parameter is determined with an accuracy of \pm 0.06 .

The error in Scherrer particle size is dependent on the FWHM which strongly depends on the peak fit: Gaussian or Lorentzian. Scherrer particle sizes are calculated based on both kinds of fits. The corresponding error is determined to be $\pm 2 \mathrm{~nm}$. 


\section{Appendix 5: Estimation of lattice strain and peak broadening}

Mean strain e $=2 *\left(\mathrm{a}_{\mathrm{FCC}}-\mathrm{c}_{\mathrm{L} 10}\right) /\left(\mathrm{a}_{\mathrm{FCC}}+\mathrm{c}_{\mathrm{L} 10}\right)$.

This represents the largest strain possible in the $\mathrm{L1}_{0}$ particles if they were fully ordered. For the $\mathrm{Co}_{41.7} \mathrm{Pt}_{58.3}, \quad \mathrm{a}_{\mathrm{FCC}}=3.79 \AA$ and $\mathrm{c}_{\mathrm{L} 10}=3.773 \AA$, giving $\mathrm{e}=0.004$.

This strain leads to an angular spread in the peak, $\Delta \theta=\mathrm{e}^{*} \tan (\theta)$, where $\theta$ is in radians.

For the (001) peak used for determining the Scherrer particle size, $2 \theta \approx 23.7^{\circ}$ and the corresponding strain broadening, $B_{\text {strain }}$ in Eq. 3.16, is twice the angular spread, $2 * \Delta \theta=0.05^{\circ}$. As seen in Table 3.3, strain broadening has a small impact on the Scherrer particle size. The effect of strain on lattice parameters is much larger, due to a linear relationship between the two. 


\section{Appendix 6: Estimation of microstructural lengthscales}

Lines were drawn on relevant micrographs and the microstructural lengthscale was determined as the average of the dark/bright contrast. Zooming in the micrographs will facilitate easier viewing of lines superposed on micrographs for line-intercept analysis.

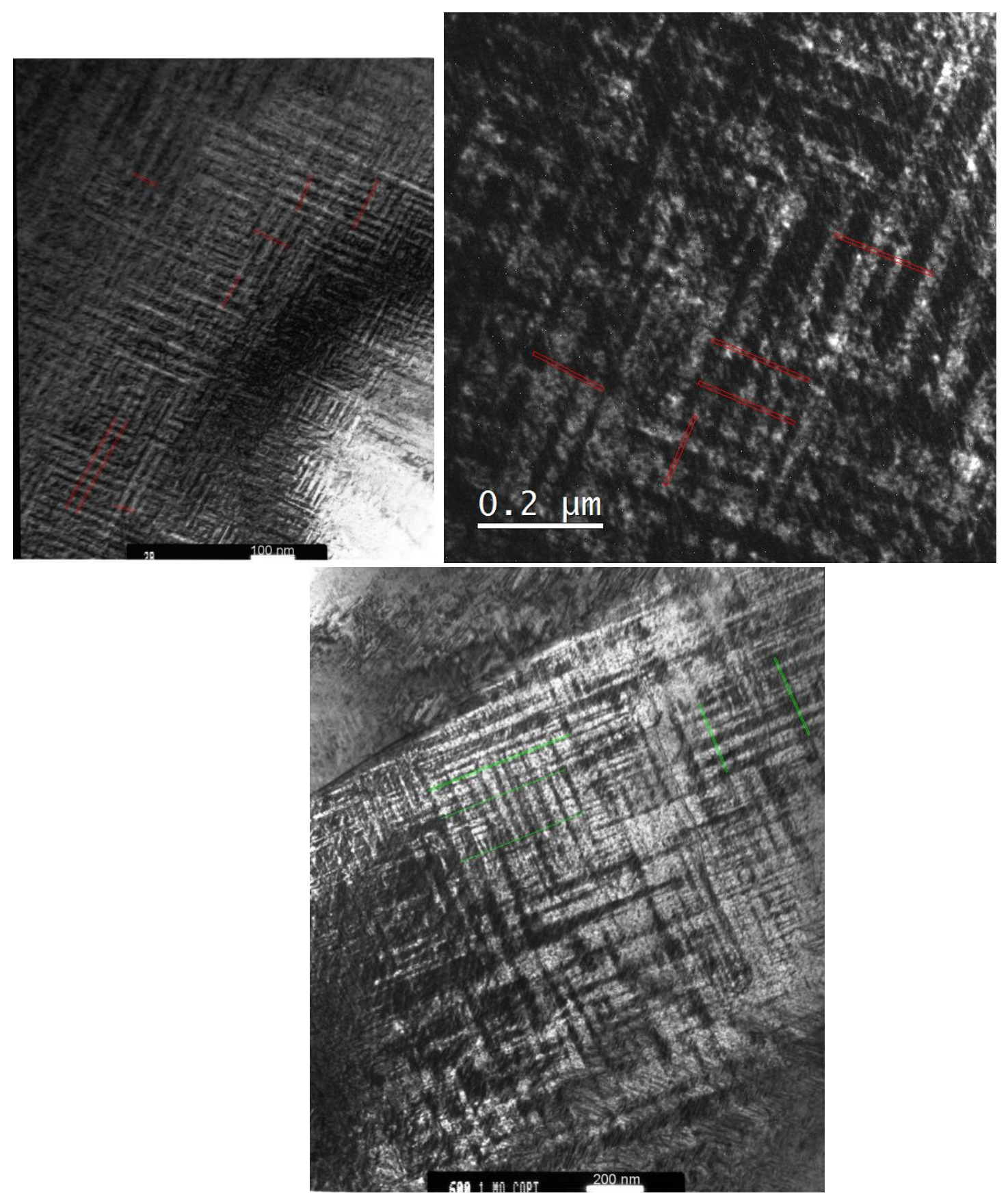

Figure A6.1: Micrographs used for estimating microstructural lengthscale for sample annealed at $600{ }^{\circ} \mathrm{C}$ for 720 hours. 


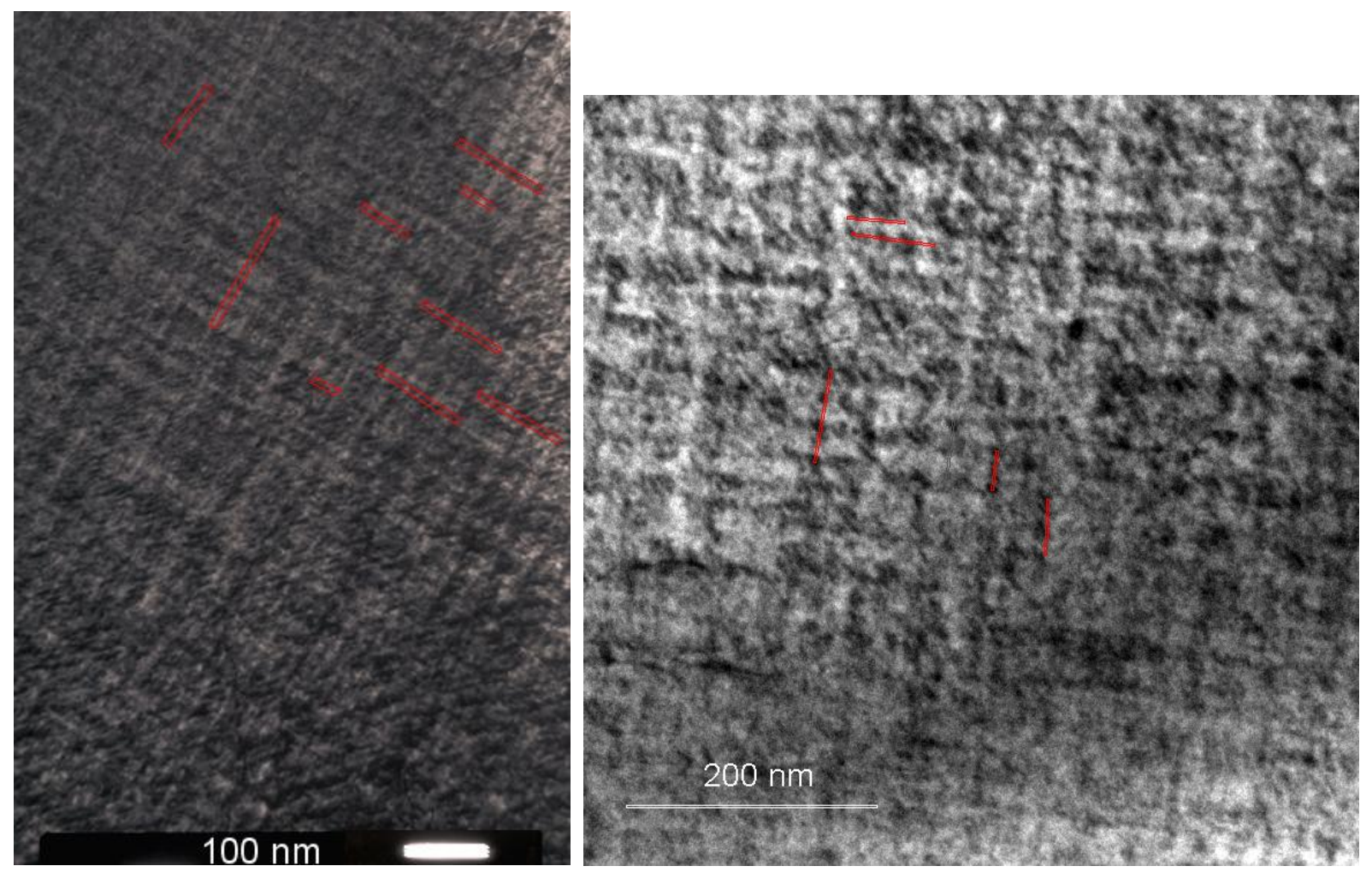

Figure A6.2: Micrographs used for estimating microstructural lengthscale for sample annealed at $600{ }^{\circ} \mathrm{C}$ for 96 hours.

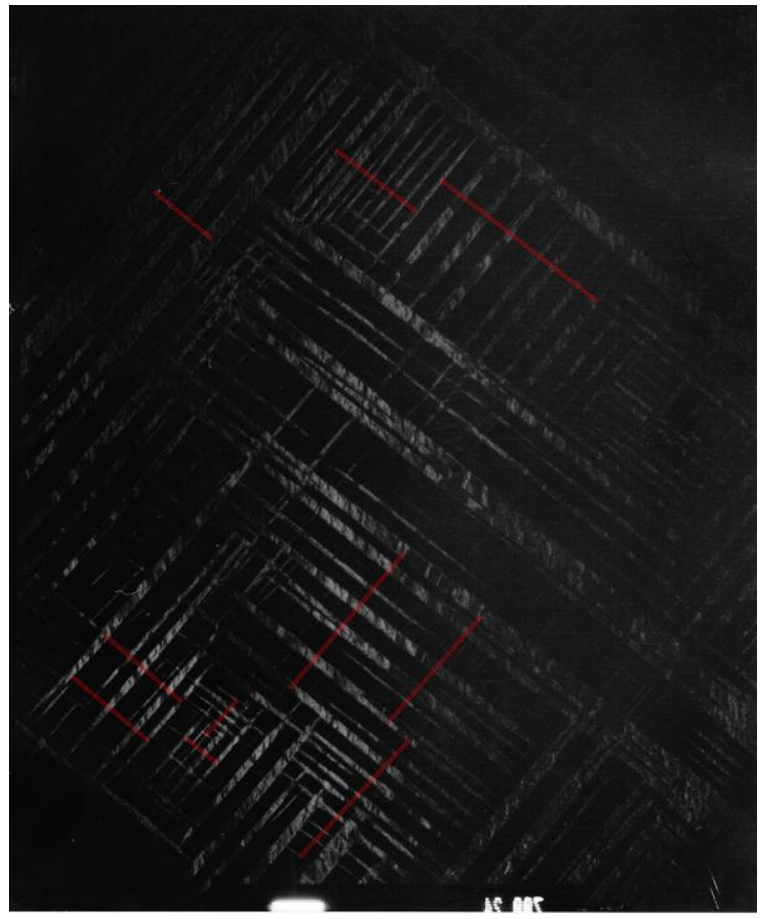

Figure A6.3: Micrograph used for estimating microstructural lengthscale for sample annealed at $700{ }^{\circ} \mathrm{C}$ for 24 hours. 


\section{Appendix 7: Comparison of peak hardness samples}

- A comparison of the M-H loops of peak hardness samples from compositions lying outside and on the eutectoid isotherms, respectively $\mathrm{Co}_{61.7} \mathrm{Pt}_{58.3}$ and $\mathrm{Co}_{40.2} \mathrm{Pt}_{59.8}$, is presented in Figure 5.1. The former evidenced superior coercivity, remanence/saturation ratio and $\mathrm{BH}_{\max }$. The peak hardness $\mathrm{Co}_{61.7} \mathrm{Pt}_{58.3}$ sample exhibited a coercivity about twice that of the peak hardness chessboard sample $\left(\mathrm{Co}_{40.2} \mathrm{Pt}_{59.8}\right)$.

- From the initial magnetization behavior of the two samples in Figure A7.1, we see that the peak hardness $\mathrm{Co}_{41.7} \mathrm{Pt}_{58.3}$ sample showed stronger domain wall pinning behavior, by $\mathrm{L} 1_{0}$ particles, compared to domain wall pinning in the peak hardness $\mathrm{Co}_{40.2} \mathrm{Pt}_{59.8}$ chessboard, by APBs.

- Figure A7.2 shows a comparison of the susceptibility curves from these two samples. The peak hardness chessboard sample showed two peaks: one from the uncoupled FCC phase at zero field and the other from the exchange coupled $\mathrm{L}_{2}$ and $\mathrm{L}_{0}$ phases at the coercivity. The peak hardness $\mathrm{Co}_{61.7} \mathrm{Pt}_{58.3}$ sample showed single phase behavior.

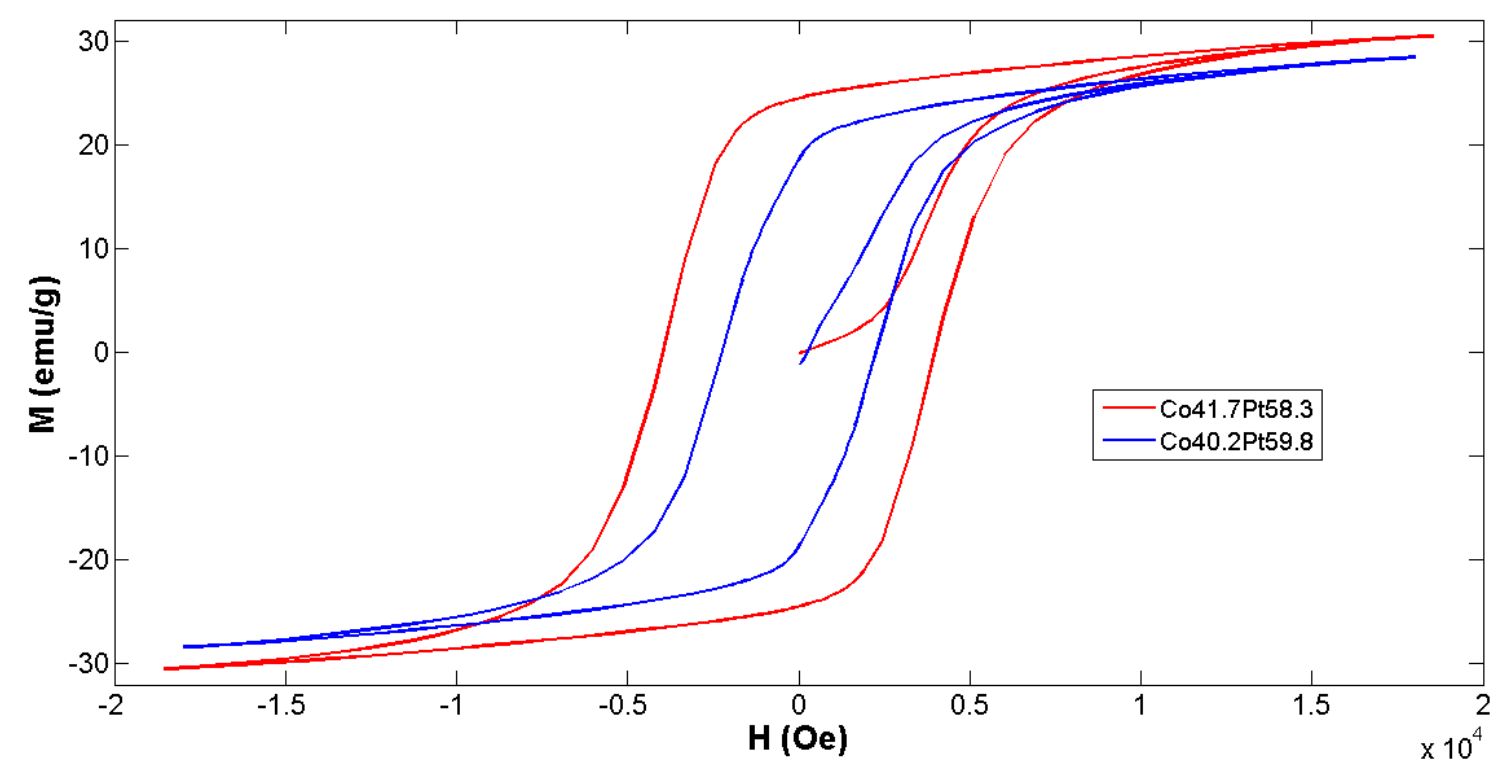

Figure A7.1: $\mathrm{M}-\mathrm{H}$ loops of the peak hardness $\mathrm{Co}_{61.7} \mathrm{Pt}_{58.3}$ alloy, which lies outside the eutectoid isotherm, and the peak hardness $\mathrm{Co}_{40.2} \mathrm{Pt}_{59.8}$ alloy, which lies on the eutectoid isotherm and exhibited the nanochessboard structure. 


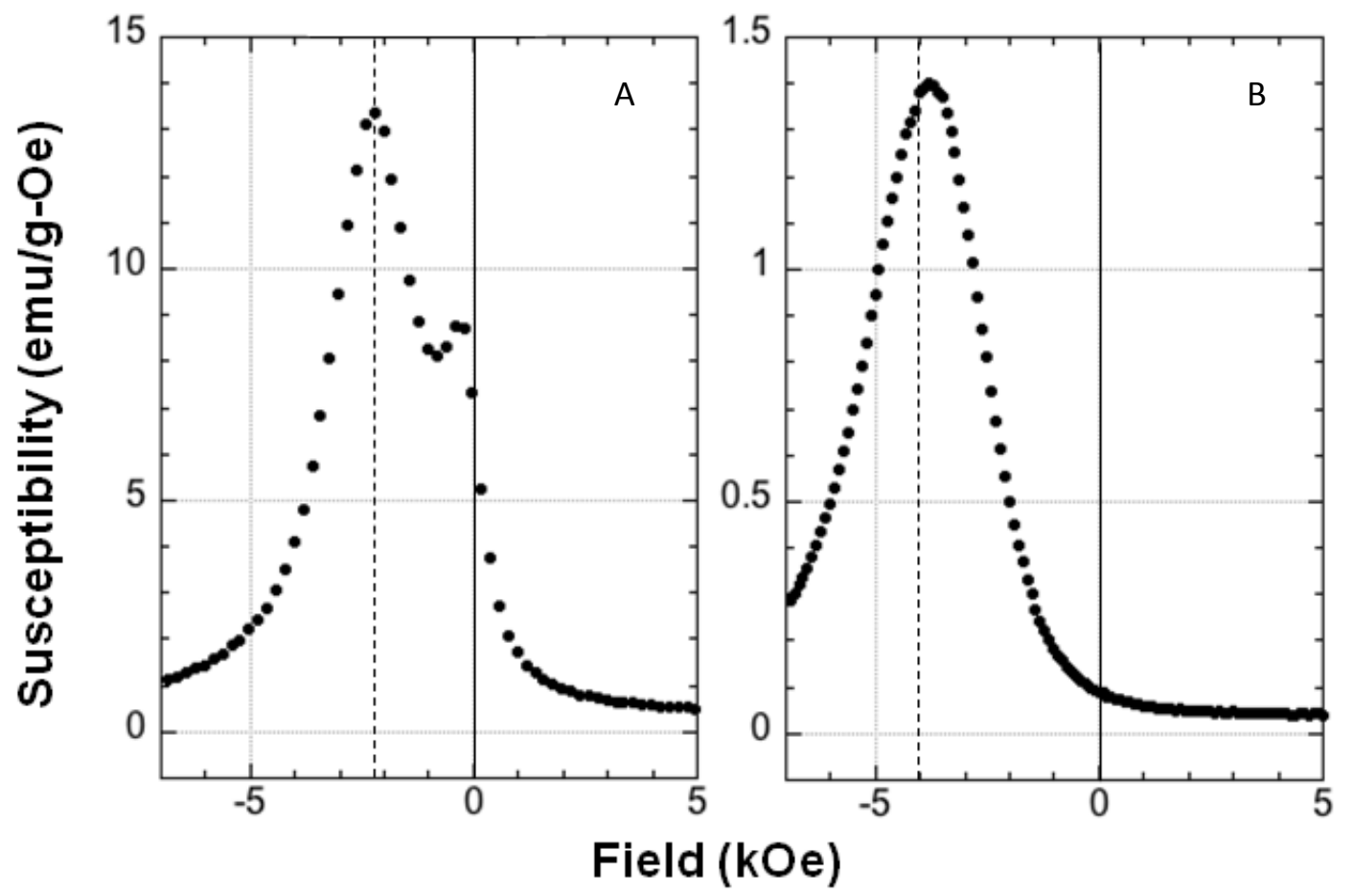

Figure A7.2: Susceptibility curves from peak hardness (A) $\mathrm{Co}_{40.2} \mathrm{Pt}_{59.8}$ chessboard and (B) $\mathrm{Co}_{61.7} \mathrm{Pt}_{58.3}$ samples. 


\section{Appendix 8: Alternate processing of $\mathrm{Co}_{40.2} \mathrm{Pt}_{59.8}$ alloys}

In addition to continuous cooling, $\mathrm{Co}_{40.2} \mathrm{Pt}_{59.8}$ alloy samples have also been processed via:

(a) Direct annealing, where quenched samples were isothermally annealed in the two-phase region without being slow cooled and

(b) Step cooling in step-wise decrements of $20{ }^{\circ} \mathrm{C}$ every 12 hours from $750{ }^{\circ} \mathrm{C}$ to $600{ }^{\circ} \mathrm{C}$, followed by annealing at $600{ }^{\circ} \mathrm{C}$ for 2 weeks.

The values of $\mathrm{H}_{\mathrm{c}}$ and $\mathrm{M}_{\mathrm{r}} / \mathrm{M}_{\mathrm{s}}$ attained upon direct annealing at $600{ }^{\circ} \mathrm{C}$ for 1 week are similar to those obtained upon continuous cooling from $750{ }^{\circ} \mathrm{C}$ to $600{ }^{\circ} \mathrm{C} @ 40{ }^{\circ} \mathrm{C} /$ day followed by a 1 week aging treatment. On the other hand, direct annealing for 2 weeks produced a higher $\mathrm{H}_{\mathrm{c}}$, 2500 Oe and $\mathrm{M}_{\mathrm{r}} / \mathrm{M}_{\mathrm{s}}, 0.68$, compared to continuous cooling followed by a 2-week aging treatment, wherein these properties were 900 Oe and 0.5, respectively. Step cooling followed by a 2-week aging treatment resulted in a coercivity of 2000 Oe and remanence to saturation ratio of 0.62 , but did not did not produce the chessboard microstructure, as can be seen from Figure A3.1. 


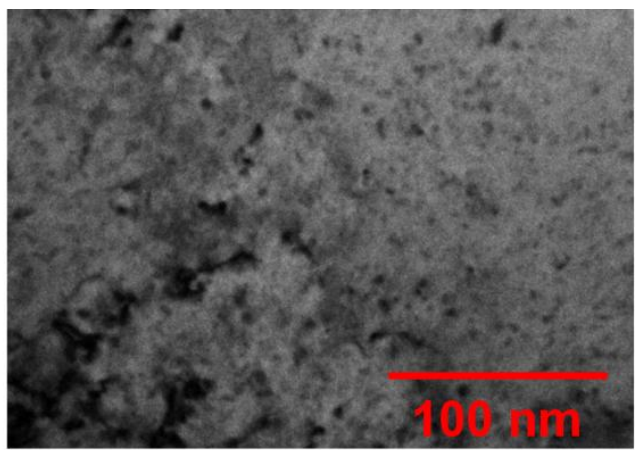

Step cooled, annealed 2 weeks at $600{ }^{\circ} \mathrm{C}$ : BF Image

Continuously cooled, annealed 2 weeks at $600{ }^{\circ} \mathrm{C}$ : BF Image

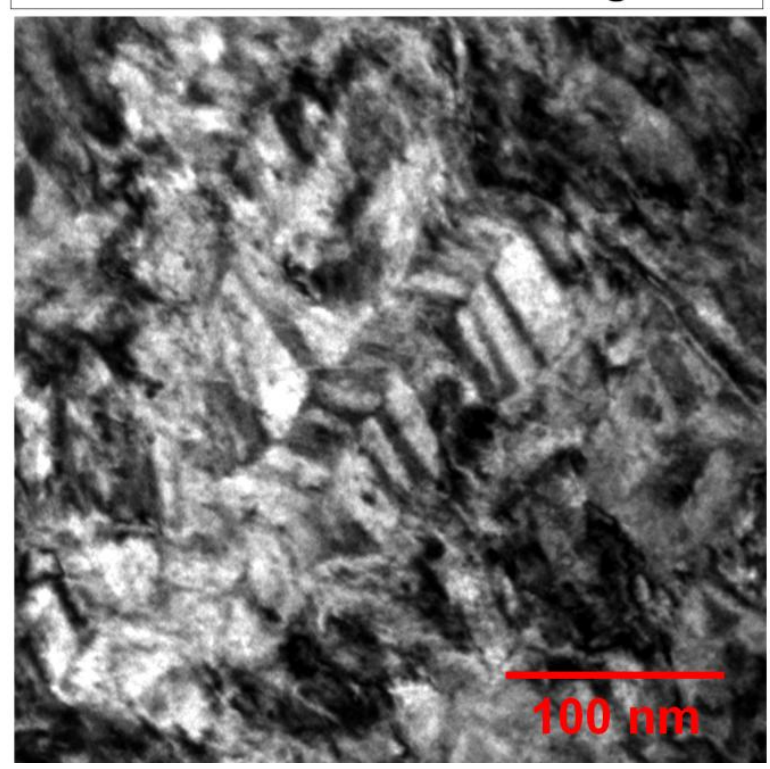

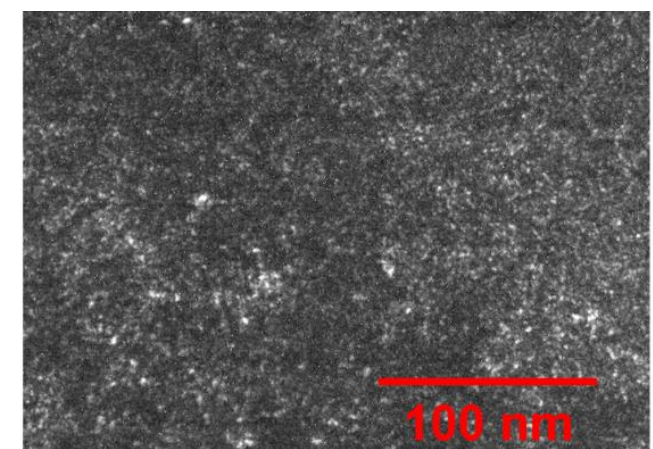

Step cooled, annealed 2 weeks at $600{ }^{\circ} \mathrm{C}$ : DF Image, $\mathbf{g}=110$.

Continuosly cooled, annealed 2 weeks at $600{ }^{\circ} \mathrm{C}$ : DF Image, $\mathbf{g}=110$.

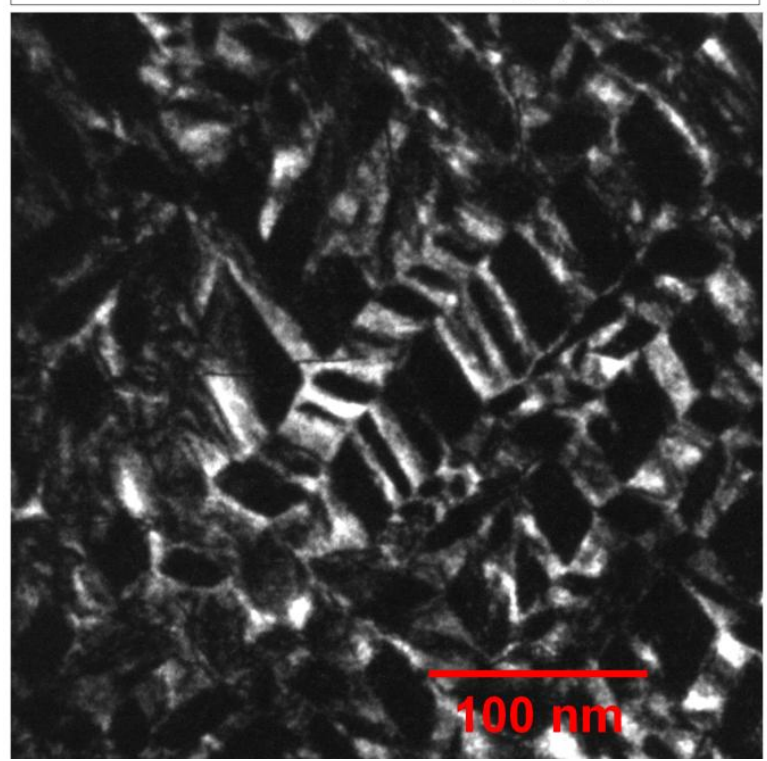

Figure A8.1: Comparison of microstructures of $\mathrm{Co}_{40.2} \mathrm{Pt}_{59.8}$ samples step cooled and continuously cooled from 750 to $600{ }^{\circ} \mathrm{C}$ and then held for 2 weeks at $600{ }^{\circ} \mathrm{C}$. 